\title{
PHOTOINDUCED PROCESSES IN NANOASSEMBLIES AND ON SURFACES
}


This research was supported by NanoNed, a national nanotechnology program coordinated by the Dutch Ministry of the Economic affairs (Project number AMM 7010).

\section{nano ned}

Photoinduced Processes in Nanoassemblies and on Surfaces

Srinidhi Ramachandra

Thesis University of Twente, Enschede, The Netherlands

ISBN: 978-90-365-3000-2

Printed at

Ipskamp Drukkers B.V., Josink Maatweg 43, 7545 PS, Enschede,

The Netherlands, http://www.ipskampdukkers.nl

(C) Srinidhi Ramachandra 2010

No part of this work may be reproduced by print, photocopy or any other means without the permission in writing of the author. 


\section{PHOTOINDUCED PROCESSES IN NANOASSEMBLIES AND ON SURFACES}

\section{DISSERTATION}

to obtain

the degree of doctor at the University of Twente, on the authority of the rector magnificus, prof.dr. H. Brinksma, on account of the decision of the graduation committee, to be publicly defended on Thursday $25^{\text {th }}$ of March 2010 at 15.00

by

Srinidhi Ramachandra

born on $17^{\text {th }}$ October 1980

in Bangalore, India 
This thesis is approved by

Promoters: $\quad$ Prof. Dr. Ir. D. N. Reinhooudt

Prof. Dr. Luisa De Cola 
|| yadeva vidyayã karoti, śraddhayopanişada, tadeva viryavattaram bhavati\|

\section{Chãndogya Upanişad}

What is done with knowledge, conviction, and meditation, will alone achieve maximum energy and efficiency 


\section{Table of Contents}

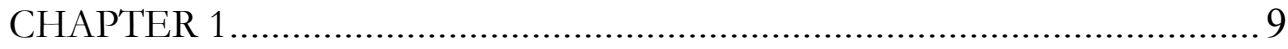

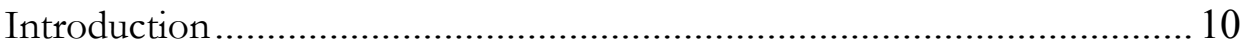

Section 1a - Nanocrystal Quantum Dots (NQDs) .................................. 13

Section $1 \mathrm{~b}$ - Transition metal complexes ............................................. 28

Section 1c - Photoinduced processes ....................................................... 32

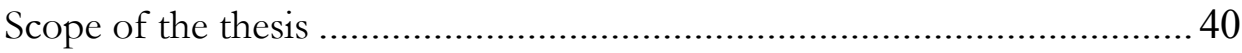

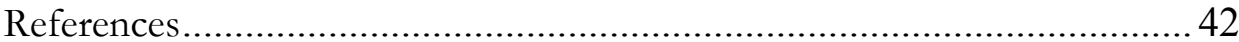

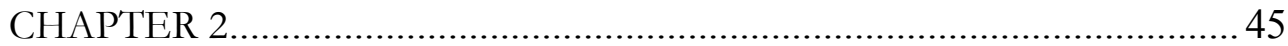

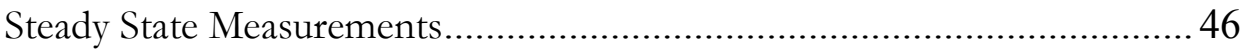

Time-Resolved Spectroscopy ……….....................................................53

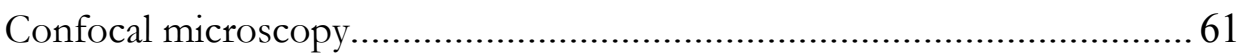

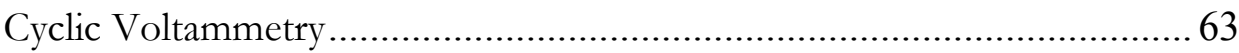

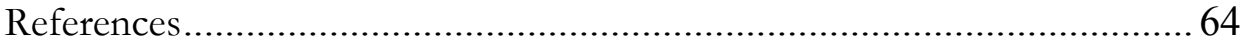

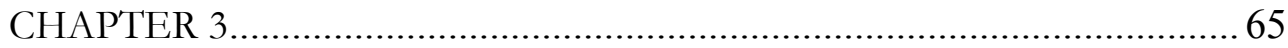

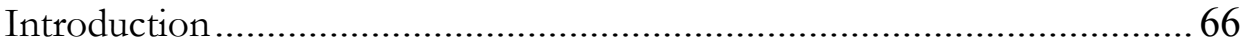

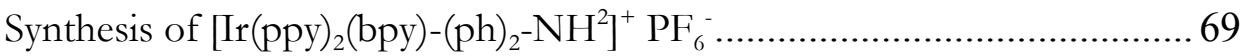

Photophysical properties of $\left[\operatorname{Ir}(\mathrm{ppy})_{2}(\mathrm{bpy})(\mathrm{ph})_{2} \mathrm{NH}_{2}\right]^{+} \mathrm{PF}_{6}^{-} \ldots \ldots \ldots \ldots \ldots \ldots \ldots . . . . . . . . .70$

Energy transfer studies in $\mathrm{CdTe} / \mathrm{Ir}-\mathrm{NH}_{2}$ nanoassemblies ......................... 77

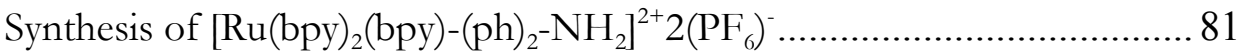

Energy transfer studies in $\mathrm{CdSe} / \mathrm{ZnS}-\mathrm{Ru}-\mathrm{NH}_{2}$ nanoassemblies ............. 91

Förster Resonance Energy Transfer (FRET) discussion ............................ 96

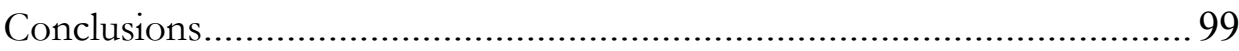

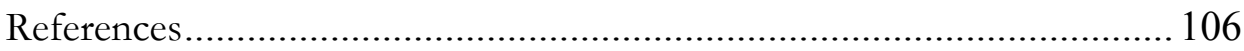

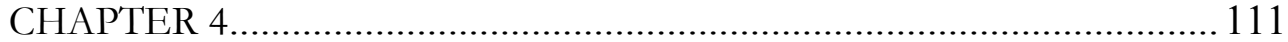

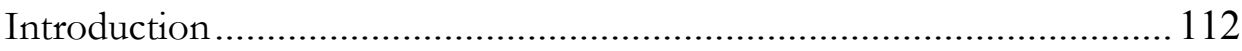




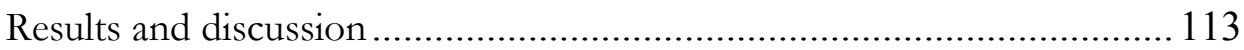

Photophysical characterization in solution.......................................... 115

Electrochemical measurements ......................................................... 124

Conductivity measurements using EGaIn setup ................................. 127

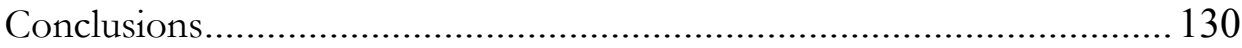

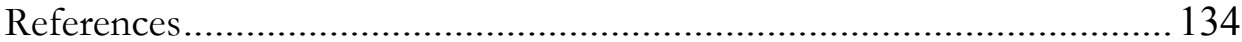

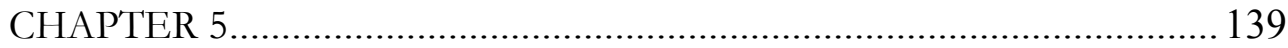

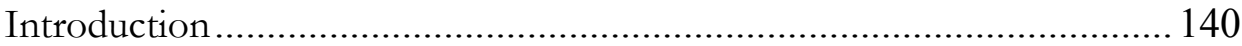

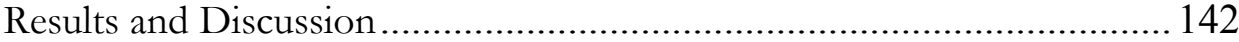

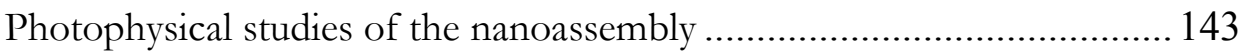

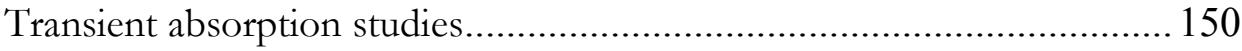

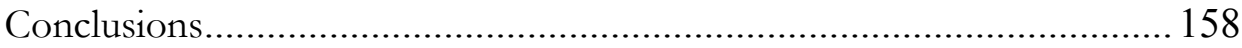

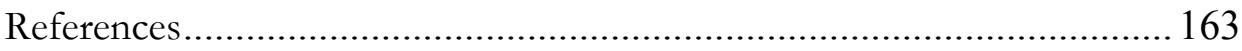

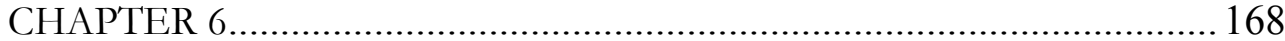

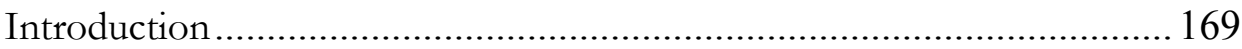

Interaction of quantum dots with zeolites ............................................. 174

CdSe/ZnS - thionine loaded zeolite L ................................................ 178

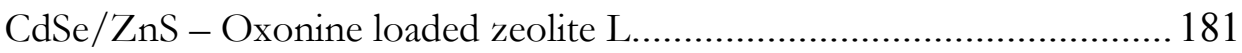

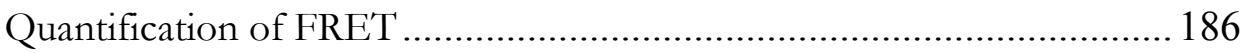

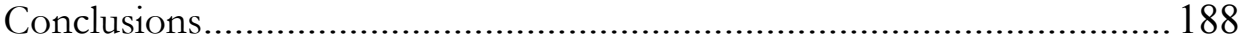

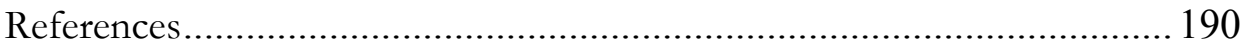

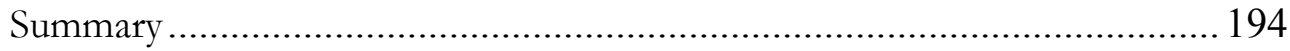

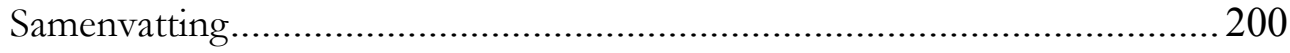

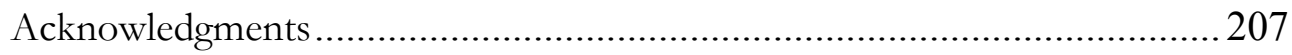

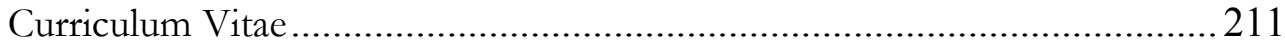




\title{
CHAPTER 1
}

\section{GENERAL INTRODUCTION}

\begin{abstract}
This chapter gives a brief introduction to the different systems described in this thesis and is divided into two parts. The first part deals with the fundamental size dependent properties of quantum dots and the consequence of quantum confinement on photophysical properties of nanocrystal quantum dots (NQDs). A brief overview of QD surface-ligand interaction is presented. The second part of this chapter deals with the basic photophysics of transition metal complexes with $\mathrm{d}^{6}$ configuration. In the concluding part of this chapter, a brief description of some of the important photoinduced processes that are encountered in this thesis is presented. The chapter concludes with a note about the scope of this thesis.
\end{abstract}




\section{Introduction}

Interaction of light with molecules results in a host of possible outcomes like driving a chemical reaction, inducing conformational changes, energy and charge redistribution, luminescence etc. The study of such interactions can be termed "photochemistry" and has been of interest to scientists for a long time. Upon absorbing a photon, a molecule might undergo various changes which are characteristic of the molecule. Hence photochemistry is a powerful tool in not only studying the light induced transitions in molecules, but also about the structural properties of the molecules. The term "photo" could be restrictive, but is used more from a historical perspective. The light matter interactions are referred to in general for interaction of electromagnetic radiation with molecules. However, in this thesis, the focus is on the photoinduced processes that occur upon absorption of ultraviolet (UV) and visible radiation by both organic and inorganic chromophores. ${ }^{1-2}$ Absorption of light causes an electron in an occupied molecular orbital which is lower in energy to 'jump' into a previously unoccupied molecular orbital at higher energy. This 'excitation' gives rise to an electronically excited state which is the heart of all photoinduced processes. A prerequisite for this electronic excitation is the energy matching between the incident photon and the energy difference between the ground and excited state. Various photophysical processes are possible after the formation of the electronically excited state depending on the molecule that absorbs light. The most common processes are excitation into higher singlet states, internal conversion, fluorescence, intersystem crossing, phosphorescence, etc.

A simple state diagram that is convenient in representing the most common events that precede the light absorption is the Jablonski diagram (figure 1.1). Upon absorbing a photon, the molecule can be electronically excited to higher excited states of same spin multiplicity. In other words, from the ground state, $\mathrm{S}_{0}$ (singlet ground state), the molecule can be excited to $\mathrm{S}_{\mathrm{n}}$ (singlet excited state). From this state different deactivation pathways are possible for the excited molecule. The first deactivation step involves non radiative relaxation of the molecule to the lowest vibrational state of the lowest excited singlet state, $\mathrm{S}_{1}$. This process of vibrational relaxation is called internal conversion 
(IC). In solution, this process occurs by transferring the excitation energy in form of heat to the solvent molecules. The time scale of this process is of the order of a few picoseconds. A second pathway involves a deactivation from $S_{1}$ to $S_{0}$ state, through an emission of photon. This $S_{1} \rightarrow S_{0}$ transition is called fluorescence. This transition is symmetry allowed and occurs on the time scale of about $10^{-10}-10^{-7} \mathrm{~s}$. Usually the molecules fluoresce from $\mathrm{S}_{1}$ state, this rule being known as "Kasha's rule". However, there are certain exceptions for this rule. One key feature of fluorescence is that the lowest transition or the $0-0$ transition is the same for absorption and fluorescence. But the emission occurs at lower energy than the absorption due to the energy loss in the excited state via solvent interactions.

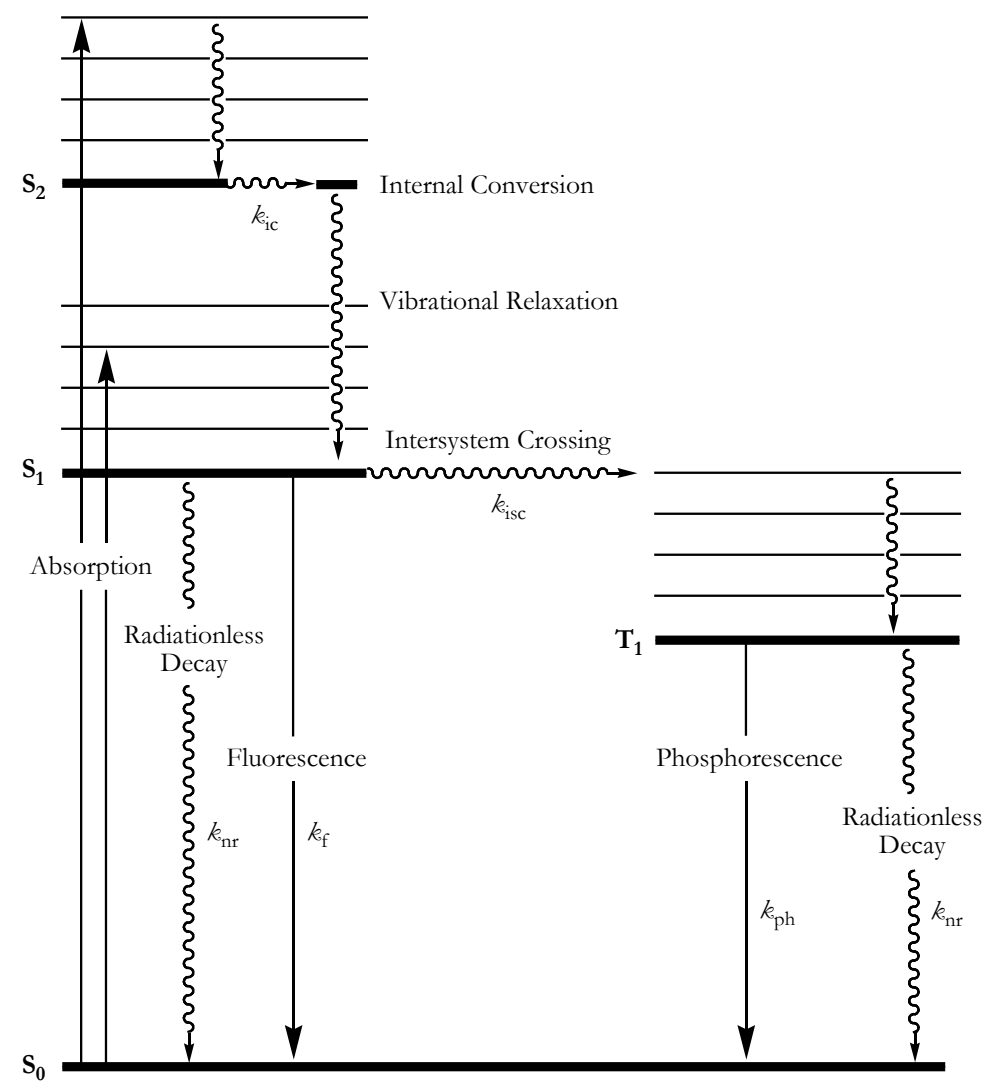

Figure 1.1: Simplified Jablonski diagram representing different electronic transitions and their respective rate constants. 
A third deactivation pathway is probable in some molecules from $S_{1}$ through an intersystem crossing (ISC) to a $\mathrm{T}_{1}$ state. Intersystem crossing is a non radiative process that occurs between two isoenergetic states with different spin multiplicities. This process is spin forbidden and occurs on time scale of $10^{-7}-10^{-9} \mathrm{~s}$ and hence ISC and fluorescence are competing processes. The deactivation from $T_{1}$ to $S_{0}$ is known as phosphorescence. This transition is forbidden, but may be observed due to spin-orbit coupling. These processes are hence relatively slow and occur on timescales of $10^{-6}-1 \mathrm{~s}$.

In this thesis, photoinduced processes in nanoassemblies based on transition metal complexes and nanocrystal quantum dots are explored. A brief description of the photoinduced transitions in each of the constituents is explained in this chapter. While the photochemistry of organometallic complexes involve processes that are indicated in the Jablonski diagram, the interaction of quantum dots with light involve processes that are governed by quantum confinement. In the first section, basic properties of quantum dots are presented. Further, in the scope of this thesis, the electronic transitions that occur in these systems upon photo excitation are discussed. In the second section, the photophysical properties of the transition metal complexes employed in the nanoassemblies are outlined. In particular, the different electronic transitions in complexes with $\mathrm{d}^{6}$ configuration are described. In the last part of this chapter, a general discussion about different photoinduced processes such as energy and electron transfer, which have been observed in these nanoassemblies, is presented. The chapter is concluded with a note about scope of the thesis. 


\section{Section 1a - Nanocrystal Quantum Dots (NQDs)}

\section{Introduction - Size matters}

Normally when referring to crystalline materials in solid state physics, reference to their dimensions is excluded. However, when the size of these materials approaches the limits of $10 \mathrm{~nm}$ or less, this variable (size) tends to dominate the physical properties that are not visible in the bulk materials. For instance, in $\mathrm{CdS}$, which is a semiconductor, the melting temperature of $1600{ }^{\circ} \mathrm{C}$ in its bulk state reduces to $400{ }^{\circ} \mathrm{C}$ when the size of this material is reduced to about $10 \mathrm{~nm}^{3}{ }^{3}$ The pressure required to induce a phase transformation from a four coordinated (wurtzite) to a six coordinated (rock-salt) phase in the same material increases from $2 \mathrm{GPa}$ to $9 \mathrm{Gpa}^{4}$ In addition, in these semiconductor materials, the optical band gap can be tuned; for example, for CdS, which has a fixed band gap of $2.42 \mathrm{eV}$ in bulk material, the gap can be continuously tuned between 2.5 to $4 \mathrm{eV}$ just by varying the size of the material. ${ }^{5}$ This significant change in the material's properties is a consequence of just varying the size and not the chemical composition. The behavior of such materials can be broadly attributed to two effects. First, the surface to volume ratio is much higher than their respective bulk counterparts which results in a significantly large number of surface atoms. In any material the contribution of the surface atoms to the bulk physical properties like free energy change and other thermodynamic properties is significant. Secondly these materials at the considered length scales are governed by unique quantum size effect that affects the intrinsic properties of these nanocrystalline cores.

This section of the chapter deals with the fundamental understanding of the above mentioned effects in nanosized crystalline semiconductor materials. A brief description of the band picture is presented followed by quantum effects in these nanocrystal quantum dots (NQDs). Further the influence of the surface properties of these materials and interaction with organic capping molecules are discussed, these play a significant role in their optical properties. A discussion of the optical properties of these NQDs constitutes the concluding part of this section. The consequence of these quantum effects 
from an optical point of view throws up new challenges and opportunities in the field of semiconductor nanoscale science.

\section{Quantum size effects}

The reduction of the physical dimensions of any semiconducting material results in a variation of their electrical and optical properties. ${ }^{6-9}$ This is predominantly due to the second effect mentioned above. The change in the optical behavior as a function of size in these materials can be understood by considering the band structure. As the size of a material reduces to few tens of nanometers, there is a systematic transformation of density of states which gives rise to the so called quantum size effects. For any material below a certain size threshold, substantial variation of the above mentioned properties are seen when the energy level spacing exceed the temperature $\left(k_{B} T\right)$. In case of bulk semiconductors, this transformation occurs at very large sizes 
A

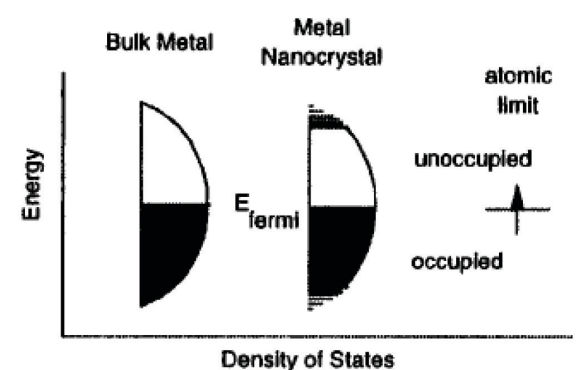

B

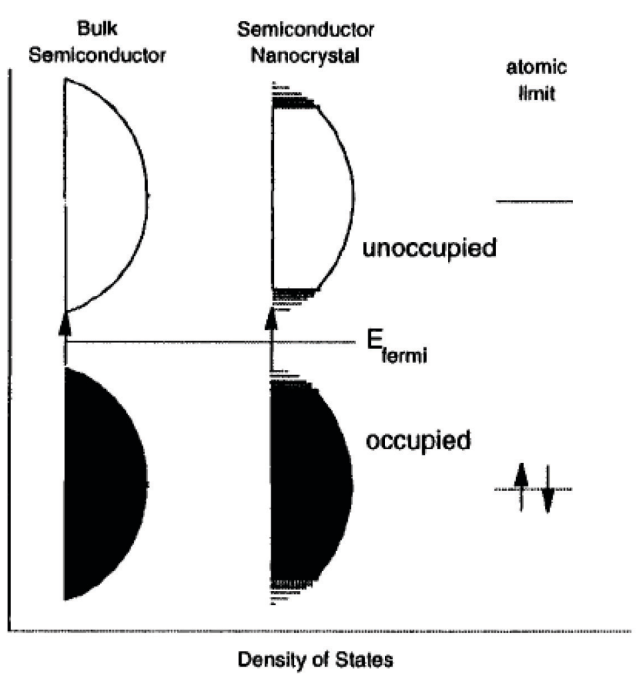

Figure 1.1: Density of states in metals (A) and semiconductor (B) nanocrystals.

(Reproduced with permission from ref 6.)

as compared to metals, insulators or molecular crystals. This can be understood by considering the band structure in solids (figure 1.1). According to the band theory of solids, bands of the solids are centered around the atomic energy levels and the width of the band indicates the extent of nearest neighbor interactions. As the size increases (from atomic limit to bulk), the centre of the band starts filling up first and the band edges fill up at the end. The size regime of the quantum dots lies in between that of a bulk material and atomic limit. In the case of metals, the Fermi level lies in the center of the band and the relevant energy level spacing (responsible for optical and electrical properties) is very small. Hence, at temperatures above few Kelvin, the optical and 
electrical properties resemble the bulk counterpart even at the sizes of few hundred atoms in the cluster. ${ }^{10}$ On the other hand, in case of semiconductors, the Fermi level lies in the band gap and hence the optical and electrical behavior of these materials is strongly dictated by the band edges. Contrary to metal nanoparticles, the optical transitions in NQDs are strongly size dependent up to the clusters of about tens of thousands of atoms.

Electrical properties in NQDs are strongly size dependent also, the energy required to add an excess charge above the band gap energy increases as opposed to being constant in bulk materials. ${ }^{11}$ The presence of one charge acts to prevent successive charge addition thereby giving rise to a phenomenon called "Columbic blockade". ${ }^{12}$ However, in this chapter, the focus is on the manifestation of the optical properties in NQDs as a result of quantum size effects that is presented in the next section.

Upon decreasing the size of the quantum dot, the electronic excitations shift to a higher energy and the oscillator strengths are clustered in a few transitions. The basic phenomenon of quantum confinement effect is a direct result of the manifestation of density of electronic states as a function of size. For bulk semiconductors, the natural length scale that governs electronic excitation is the Bohr-exciton radius (electron-hole separation), $a_{x}$, which is determined by the strength of the electron - hole coupling. Typical Bohr-exciton radius ranges between $2-10 \mathrm{~nm}$ in bulk semiconductors. However, as we consider the NQD regime $(2-10 \mathrm{~nm})$, the size range corresponds to the quantum confinement regime where the spatial extent of electronic wavefunctions is comparable with the size of the semiconductor. In case of NQDs, it is not the strength of coupling between the electron and hole wavefunctions, but it is the size of the nanocrystal that defines the spatial extent of an electron-hole pair state. As a result of this "geometrical confinement" electrons feel the particle boundaries and respond strongly to any variation in the particle size (boundary).

The quantum size effects for nanocrystals are described under first approximation using the effective mass approximation by employing a simple "quantum box" model. ${ }^{13}$ The bulk effective masses for electron and hole are 
employed in this model, which assumes spherical shape for quantum dots and the conduction and valence bands to have parabolic shapes. The detailed theoretical description of the quantum size effects and band structure of the quantum dots are beyond the scope of this thesis. However, it is interesting to consider the most important result of the effective mass approximation that can be most useful to get a physical insight into the optical properties of quantum dots. The size dependent band gap energy of the nanocrystals is expressed according to the above approximation as

$$
\begin{gathered}
E_{g}(Q D)=E_{0}+\frac{\hbar^{2} \pi^{2}}{2 m_{e h} R^{2}} \\
\text { and } \\
m_{e h}=\frac{m_{e} m_{h}}{m_{e}+m_{h}}
\end{gathered}
$$

where $E_{0}$ is the bulk band gap, $m_{e}$ and $m_{b}$ are the effective masses of electron and hole respectively and $R$ is the nanocrystal radius. From the above equations, it is clear that the nanocrystal band gap varies inversely as the square of the particle size. The direct experimental consequence of this equation, manifested as the optical properties of quantum dots are explored later in this chapter.

\section{Electronic absorption spectra of NQDs}

In this section, the optical properties of quantum dots are discussed by considering CdSe, the prototype NQD and CdTe NQDs. One of the most noticeable effects due to quantum confinement would be the change in the electronic absorption spectra as a function of size. Smaller particles exhibit optical transitions at higher energy as compared to the bigger nanocrystals. ${ }^{14}$ This is demonstrated in figure 1.2. 


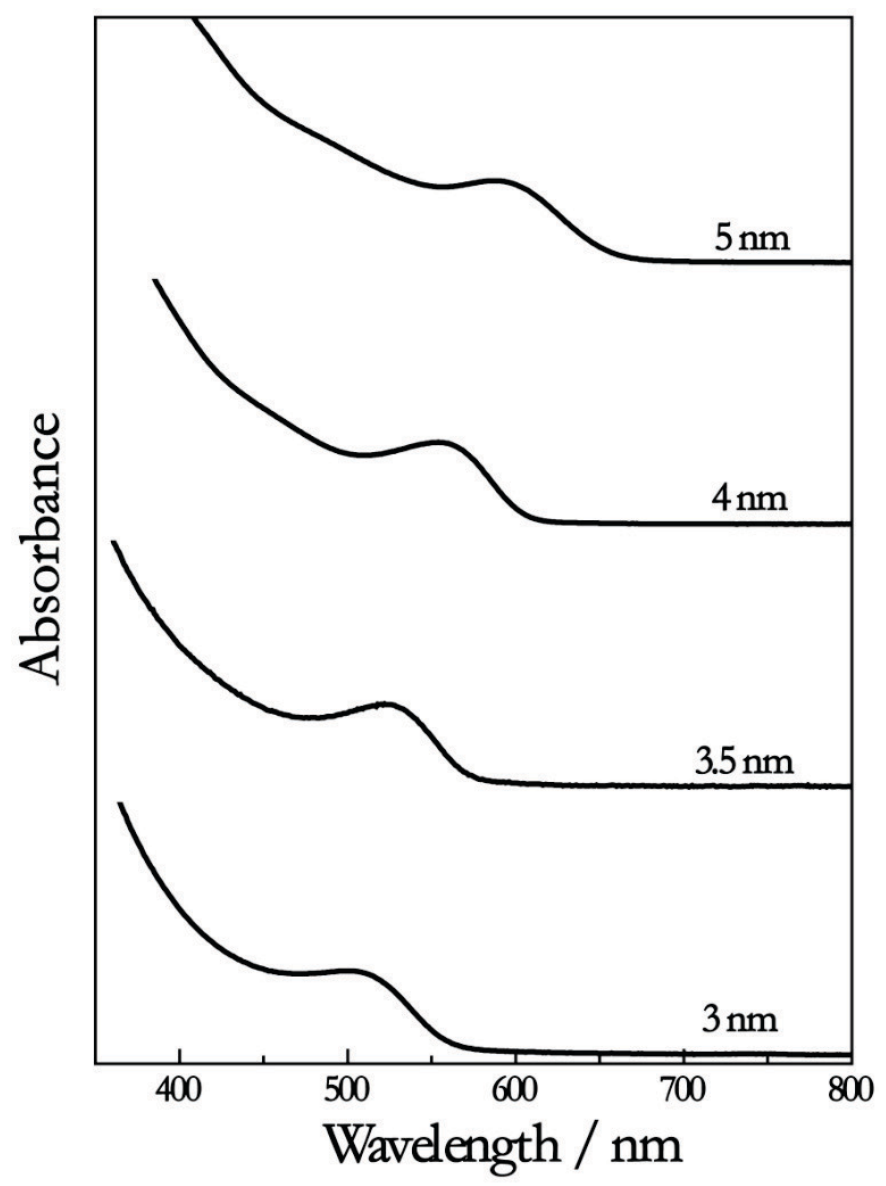

Figure 1.2: Absorption spectra of CdTe NQD with different crystal diameters

As discussed in the previous section, for the nanocrystal quantum dots, due to the quantum confinement, the band structure ceases to exist and the energy levels are discretized. These quantized states can be represented by employing two quantum numbers, $\mathrm{L}$ and $n,{ }^{13}$ similar to that used for atomic transitions. $\mathrm{L}$ represents the angular momentum of the envelope wave function which describes the motion of charge carriers in the confined potential of the nanocrystal, whereas $n$ denotes the number of state in a series of states with a given symmetry $(\mathrm{L})$. In the above notation, the different quantized states are represented with letters such as $\mathrm{S}$ (for $\mathrm{L}=0), \mathrm{P}(\mathrm{L}=1), \mathrm{D}(\mathrm{L}=2)$ and so on. At 
the outset, the electronic absorption spectrum of NQD seems to be characterized by an absorption band that originates due to the promotion of an electron from the valence band edge to the conduction band edge at energy corresponding to the band gap energy. However, this is not the case in practice. As can be seen from figure 1.3, the absorption spectrum of CdSe quantum dots $(\mathrm{R}=4.1 \mathrm{~nm})$, exhibits many features. ${ }^{15}$ In nanocrystals, the quantum confinement gives rise to band mixing effects that complicate the spectra. Ekimov and coworkers have calculated the size dependent hole states for $\mathrm{CdSe}^{16}$ and figure 1.3 (a) represents the first three states according to this calculation. The arrows represent different allowed transitions in these NQDs. The right panel of figure 1.2 presents a linear electronic absorption spectrum of $4.1 \mathrm{~nm}$ NQDs. All the absorption features are attributed to different transitions based on comparisons with theoretical calculations. The band edge absorption is denoted by $1 \mathrm{~S}(\mathrm{e})-1 \mathrm{~S}(\mathrm{~h})_{3 / 2}$ where $3 / 2$ represents the spin angular momentum of the hole state. The fact that the absorption spectrum is structured reveals the high quality of nanocrystal samples with very high monodispersity which could be synthesized by using the solvothermal synthesis methods. 
a

CdSe NC

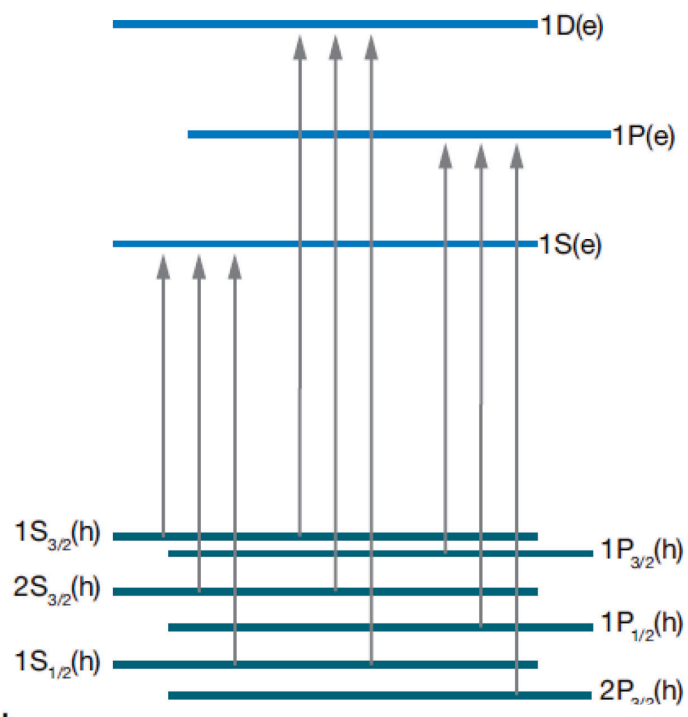

b

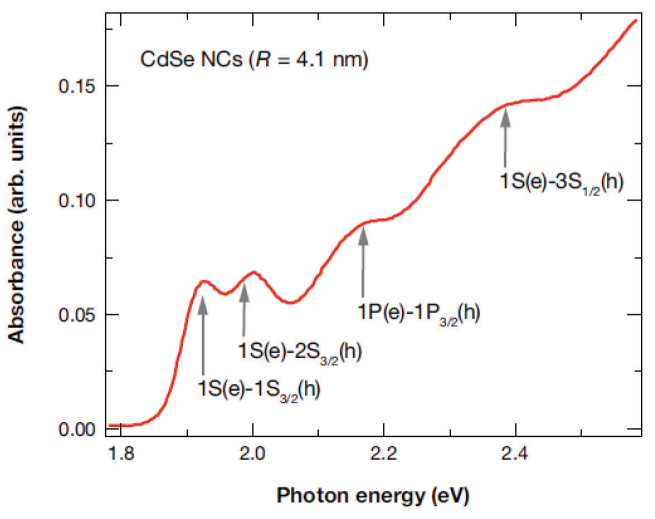

Figure 1.3: (a) Different electron and hole states in CdSe and the arrows indicated the different allowed electronic transitions in CdSe. (b) Linear absorption spectrum of CdSe NQDs with assignments of bands (Reproduced with permission from ref 15) 


\section{Exciton dynamics and Emission properties of NQDs}

The first three hole states in CdSe are useful in understanding and assigning the three different electronic transitions involved in NQDs and hence understanding the absorption spectrum of NQD. In order to understand complete exciton dynamics, time resolved absorption and emission spectroscopies are employed. Transient absorption spectroscopy is an extremely powerful tool in addressing the charge separation and recombination dynamics in semiconductor nanoscience ${ }^{17}$. It allows us to monitor the pump induced absorption changes as a function of time. For NQDs, it is a well known fact that upon excitation, the electron is not promoted to the band edge of the conduction band, but to its higher empty states. The electron subsequently relaxes back to the band edge state through interactions with phonons in a time scale of few hundreds of femtoseconds before recombining with the hole in the valence band and thereby generating a photon. Using transient absorption spectroscopy, both inter and intra band dynamics of the quantum dots maybe probed. ${ }^{18-19}$ Figure 1.4 represents the femtosecond transient absorption spectrum of CdSe with mean radius of $4.1 \mathrm{~nm} .{ }^{18}$ The linear absorption spectrum is also shown for comparison. 

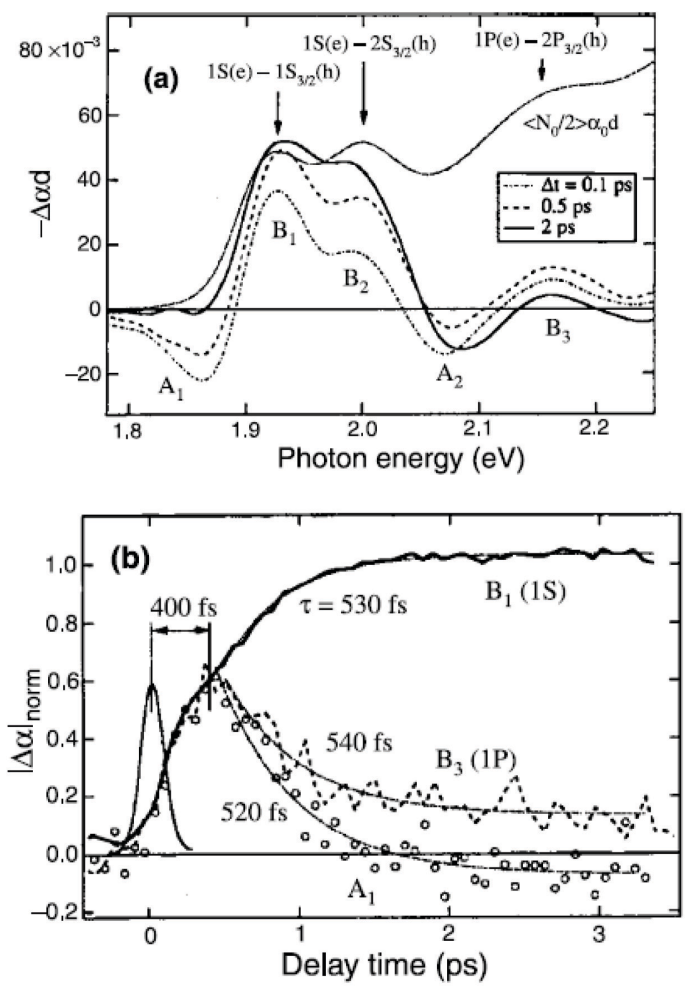

Figure 1.4: (a) Femtosecond transient absorption spectra of CdSe $(R=4.1 \mathrm{~nm})$ recorded at $0.1 \mathrm{ps}, 0.5 \mathrm{ps}$ and 2 ps delays. Linear absorption spectrum of CdSe NQDs with assignments of bands is overlaid for comparison. (b) Transient absorption kinetics at different spectral positions. (Reproduced with permission from ref 18)

The transient spectra of NQDs provide a wealth of information about the exciton dynamics in NQDs. Different features are seen in the spectra shown above. The change in the absorption spectra upon exciting with a femtosecond pulse is attributed to two main effects. First, upon excitation, "hot carriers" are generated which have a very fast relaxation time. 18,20 These carriers, due to coulombic interaction, induce a blue shift in the absorption spectrum due to the Stark effect. ${ }^{21}$ Secondly, as the hot carriers are generated, they relax at a rate much faster than the depopulation of the band edge state. As a result the number of energy levels closer to the band edge available for the relaxing charges becomes progressively diminished and subsequently the higher levels 
are filled. This "state filling effect" $21-22$ also induces a blue shift in the transient spectrum. The above two effects are manifested as the $A_{1}$ transient feature in figure 1.4 (a). Further, the bleach at $\mathrm{B}_{1}$ and $\mathrm{B}_{3}$ are present at different positions, with the kinetics of both the formation of $\mathrm{B}_{1}$ and the decay of $\mathrm{B}_{3}$ following the same kinetics (figure 1.4 (b)). These features are assigned to $1 \mathrm{~S}(\mathrm{e})-1 \mathrm{~S}(\mathrm{~h})_{3 / 2}$ and $1 \mathrm{P}(\mathrm{e})-12 \mathrm{P}(\mathrm{h})_{3 / 2}$ respectively which could also be seen by comparing the transient spectra with linear absorption spectra. The intraband relaxation of hot carriers can also occur via an "Auger process" where the electron hole recombination energy is transferred to a third particle that gets excited. ${ }^{15,18-19,23}$ This process occurs when there is more than one exciton generated per photon. Different processes that occur upon exciting a nanocrystal quantum dot are shown schematically in figure 1.5. Multiple exciton interactions are omitted for simplicity in the scheme.

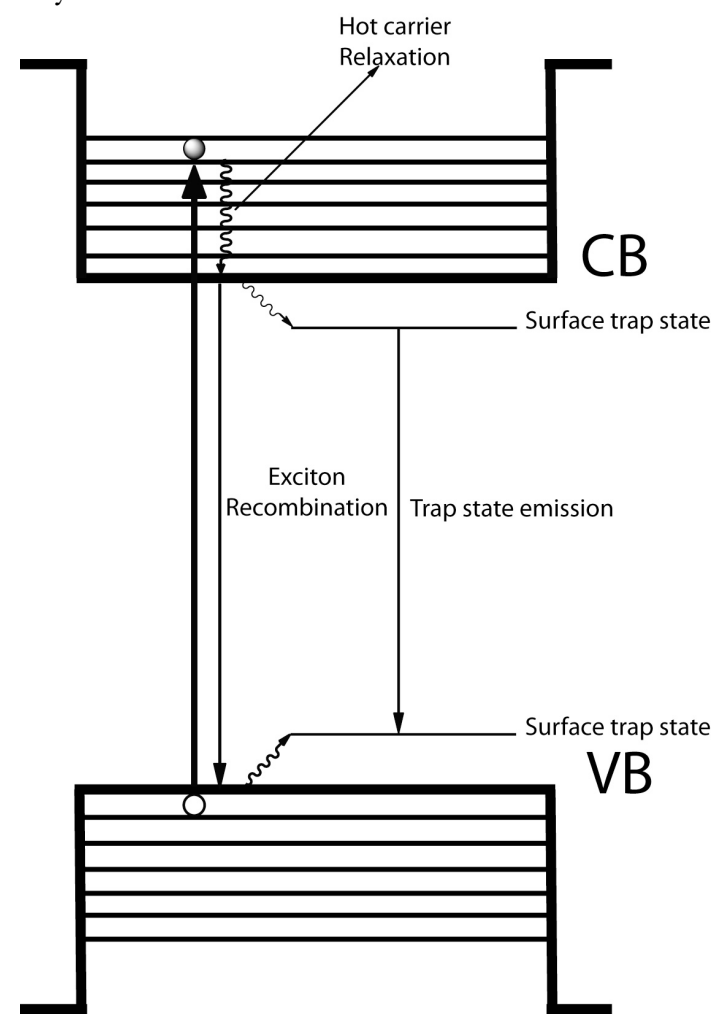

Figure 1.5: Different processes that take place in NQDs upon excitation 
The exciton recombination takes place from the band edge as shown in figure 1.5. The band edge recombination dynamics are studied using steady-state and time resolve emission spectroscopic techniques. Ideally all the excitons should recombine from the band edge. This would give rise to a narrow emission profile and a monoexponential lifetime decay. These characteristics are found in very high quality nanocrystals. However, quantum dots can have surface sites that are coordinatively unsaturated and hence give rise to defect sites. These sites can exist even in case of effective ligand coverage on the surface of the dots. In such cases, the electrons and holes get trapped due to the surface trap states that are present in the band gap. The excited state lifetime of the NQDs will be shorter and the decay profile multi-exponential in nature. Such defects contribute to the reduction of the emission quantum yields of the nanocrystals. A very high quality nanocrystal exhibits narrow intense emission and monoexponential lifetime decay of about $20 \mathrm{~ns}^{17}$. However, in presence of defect sites, the average lifetime is reduced. In fact the trap state emission can be seen in the steady-state emission spectrum, and the corresponding emission lifetimes are of hundreds of nanoseconds. Figure 1.6 presents the absorption and emission spectrum of $3 \mathrm{~nm}$ CdTe NQDs recorded in toluene. The defect emission can be at lower energies with respect to the exciton emission in the steady-state spectrum.

The fact that the absorption and emission for the NQDs lie very close is indicative of an emission arising from the direct recombination of the charge carriers from the band edge. 


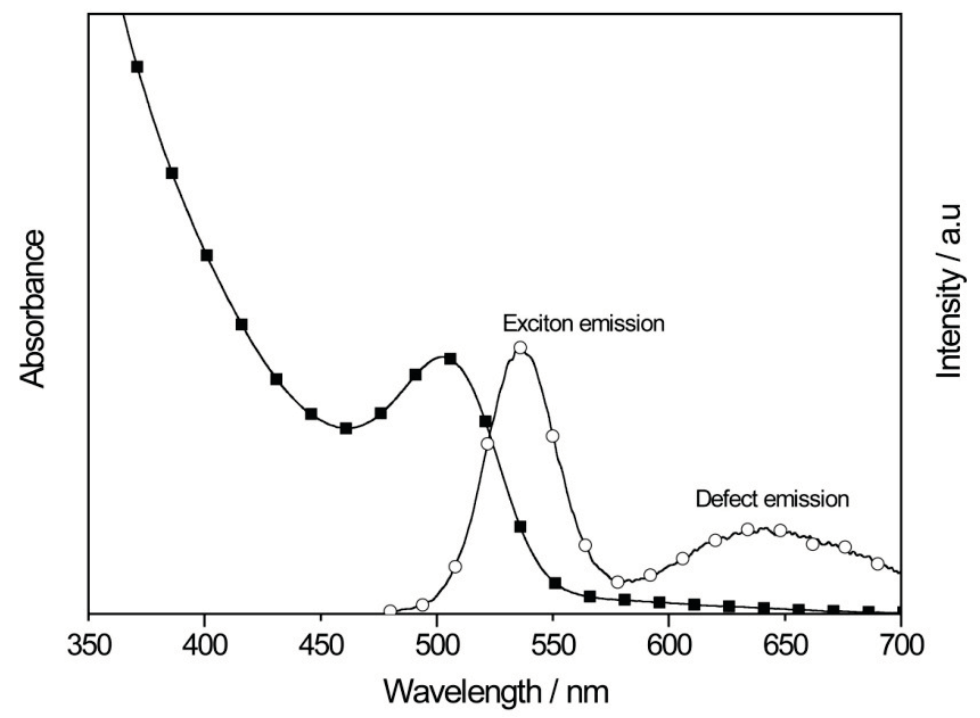

Figure 1.6: Absorption (--) and emission (-O-) spectra of CdTe (3 nm) NQDs measured in toluene. The high energy emission arises from the exciton recombination whereas the low energy band is the defect emission

The defect emission is circumvented by passivating the NQDs with a few layers of higher band gap semiconductor like $\mathrm{ZnS}^{24-26}$ This results in a core/shell configuration, which has improved emission properties. These core/shell NQDs possess higher quantum yields and are considerably more stable in normal atmosphere as opposed to the core only quantum dots which are highly sensitive to the presence of oxygen. The $\mathrm{Cd}^{2+}$ sites at the surface are oxidized in air, thereby resulting in the effective shrinkage of the NQD size which results in a blue shift in their absorption spectra. ${ }^{27-28}$ Hence from an application point of view, it is prudent to employ nanocrystals with core/shell geometries. This passivation of the dots with the shell also to a certain extent reduces the toxicity of these materials.

\section{Surface interaction in NQDs}

From a chemist's point of view, the nanocrystal quantum dot is viewed as a large molecule with tens of thousands of atoms. The development in the synthetic procedure in last two decades of NQDs has been remarkable. Most 
of the modern wet chemical synthetic techniques are based on the solvothermal route published by Murray and coworkers back in early 90's. ${ }^{29-30}$ The synthesis involves injection of the organometallic precursors into hot coordinating solvents like trioctyl phosphine (TOP) or trioctyl phosphine oxide (TOPO). The nucleation of the particles is arrested at various stages by varying the temperature thereby giving a control over the size of the nanocrystals synthesized. Nanocrystals with monodispersity as low as $5 \%$ have been synthesized through this route. The coordinating solvents used in the synthesis act as capping ligands as well. The presence of these capping ligands coordinated to the nanocrystal surface not only prevents the further nucleation and agglomeration of these particles, but also render these colloidal particles reasonably soluble in various organic solvents.

The surface ligand interaction can be understood based on a hard and soft acid base concept proposed by Ralph Pearson in 1960's. ${ }^{31}$ The NQDs core usually contains the charged metal ion like $\mathrm{Cd}^{2+}, \mathrm{Pb}^{2+}, \mathrm{In}^{3+}$ etc. These ions are termed as 'soft' acids due to their large polarizable core. Phosphonates are base conjugates of phosphonic acids and carboxylic acids, are 'hard' bases. Hence these moieties form a weaker bond with the $\mathrm{Cd}^{2+}$ ions through the lone pair of electrons on P. Hence it is easy to replace TOP through typical ligand exchange with any other molecule that contains a terminal functionality which has a less 'hard' basicity. TOPO could be replaced with amines, whose acidity is less 'hard' than $\mathrm{PO}_{3}{ }^{3-}$. Alkyl thiolates which are considered as soft acids form the strongest bond with the $\mathrm{Cd}^{2+}$ surface through the lone pair of electrons on sulfur. In fact thiols have the strongest affinity to the NQDs that have $\mathrm{Cd}^{2+}$ core. ${ }^{23}$ This ligand exchange renders these nanocrystals extremely versatile from a processability point of view. Bifunctional ligands such as mercapto acetic acid could be employed to exchange TOPO from the NQD surface wherein the thiolate binds to the QD surface and the carboxylate moiety is exposed to the outer environment. This type of ligand exchange renders NQDs water soluble and hence increases its applicability in biological sciences.

In this thesis, similar dynamic interactions between different NQDs and coordination metal complexes terminated with functional groups such as 
thiolates and amines have been explored. Such surface interactions are essential in fabricating bifunctional nanoassemblies which constitute a size dependent inorganic core and a redox active organometallic surface moiety. Photoinduced processes in such nanoassemblies comprise the central focus of this thesis work. 


\section{Section $1 b-$ Transition metal complexes}

Transition metal complexes of iridium and ruthenium have been explored in this thesis. These metal complexes possess a $\mathrm{d}^{6}$ configuration in the ground state, thereby having a closed shell electronic configuration. In other words, all the electrons are paired and the ground state has a singlet spin multiplicity. Due to the presence of a heavy atom in the complexes of both metals, the spin-orbit coupling plays a significant role in influencing their photophysical properties. Consequently intersystem crossing processes are favored in these systems from the $\mathrm{S}_{1}$ state and the predominant deactivation pathway for these systems is phosphorescence. The intersystem crossing efficiencies are almost unity which is mirrored in the absence of any fluorescence emission in these complexes. Unlike organic chromophores, they tend to have very long excited state lifetimes of the order of few microseconds in deaerated solutions. However, the lowest excited state does not have purely a triplet character, but is a state with mixing of singlet and triplet. The reason behind this is again extensive spin-orbit coupling.

It is worthwhile considering the bonding in these complexes in order to understand the frontier orbitals that are involved in various electronic transitions. Crystal field or ligand field theory gives a good explanation about the bonding involving the central metal atom and the ligands. ${ }^{32}$ According to these theories, the ligands are considered as point charges and in presence of a positively charged metal centre and as a result, an octahedral field is created due to electrostatic interactions. The five $d$ orbitals of the central metal atom are split into two sets of orbitals, namely $t_{2 g}$ (three orbitals) and $e_{g}$ (two orbitals) in this octahedral field. The splitting between these two set of orbitals is defined by the crystal field splitting parameter, $\Delta_{\circ}$. The magnitude of splitting between these two set of orbitals is influenced by various factors: the radius of the central metal ion, the charge on the metal ion and the nature of the coordinating ligands. The ligands like bipyridine (bpy) and phenanthroline (phen), which are encountered in this thesis, are strong field ligands, and the magnitude of splitting is larger. The extent of splitting induced by the ligands can be guessed with reasonable accuracy by considering the position of the 
ligand in an empirical series called the 'spectrochemical series' ${ }^{33}$ On the other hand, the nature of the central metal atom is also crucial in determining this effect. The splitting induced by bpy ligands in $\operatorname{Ir}(\mathrm{III})$ complexes are much larger than that observed for same ligands in $\mathrm{Ru}(\mathrm{II})$ complexes.

Crystal field theory treats the electronic structure of a complex as a single entity. However, as an approximation, it is convenient to represent the electronic structure by distributing the electrons (involved in bonding) into two different sets of orbitals that are localized on the central metal atom and the ligands. This molecular orbital (MO) method gives a complete picture of representing the electronic transitions in these complexes. In MO approximation, the molecular orbitals are constructed by a linear combination of orbitals of the central metal atom and the coordinated ligands. This method yields a simplified MO energy level diagram which is represented in figure 1.7 indicating different electronic transitions in $\mathrm{d}^{6}$ configuration metal complex.

$$
\text { Metal Orbitals } \quad \text { Molecular Orbitals Ligand Orbitals }
$$

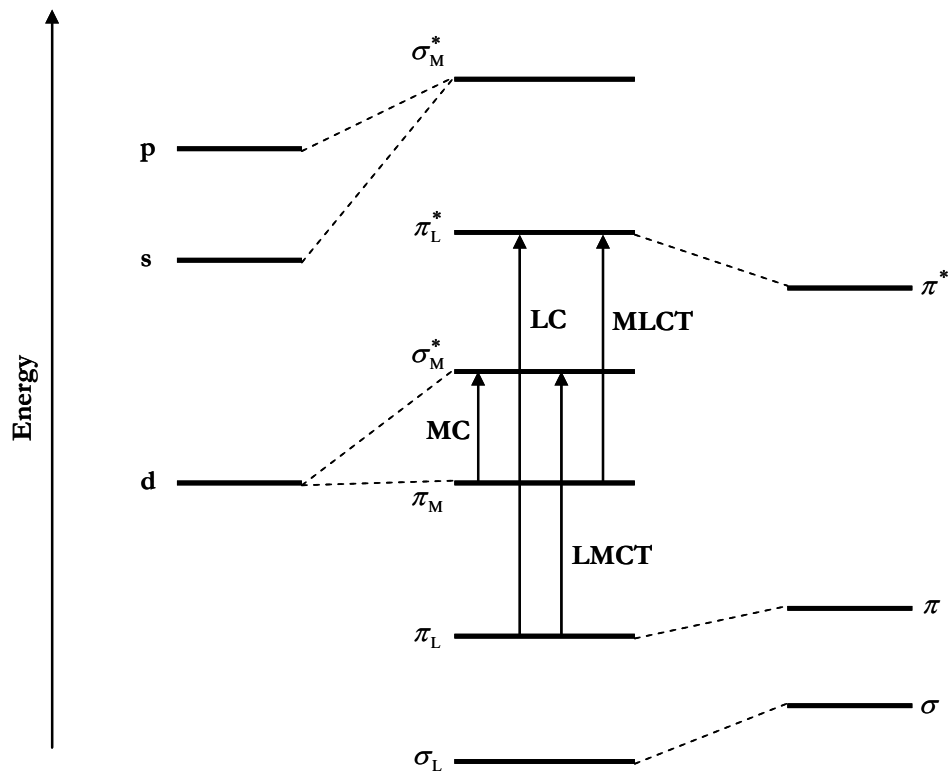

Figure 1.7: A simplified energy level diagram representing different transitions in a $\mathrm{d}^{6}$ metal complex. 
Using the abovementioned crystal field theory or ligand field theory model, four different transitions can be explained in typical $\mathrm{d}^{6}$ metal complexes. Here the $\pi_{\mathrm{M}}$ and $\sigma_{\mathrm{M}}{ }^{*}$ are the triply degenerate $t_{2 g}$ orbitals and doubly degenerate $e_{g}$ orbitals respectively.

1) Metal Centred (MC) transitions from non-bonding $\pi\left(t_{2 g}\right)$ orbitals to antibonding $\sigma^{*}\left(e_{g}\right)$ orbitals mainly localized on the central metal ion (also denoted as $d-d$ transitions).

2) Metal-to-Ligand Charge-Transfer (MLCT) transitions from non-bonding $\pi\left(t_{2 \mathrm{~g}}\right)$ or anti-bonding $\sigma^{*}\left(e_{\mathrm{g}}\right)$ orbitals centred on the metal to anti-bonding $\pi^{*}$ centered on the ligands ( $d-\pi^{*}$ transitions).

3) Ligand-to-Metal Charge-Transfer (LMCT) transitions from ligand-centred bonding $\pi$ orbitals to non-bonding $\pi\left(t_{2 g}\right)$ or anti-bonding $\sigma^{*}\left(e_{g}\right)$ orbitals centered on the metal.

4) Ligand Centred (LC) transitions between bonding $\pi$ and anti-bonding $\pi^{*}$ orbitals centered on the ligands ( $\pi-\pi^{*}$ transitions).

One important aspect to consider in theses $\mathrm{d}^{6}$ metal complexes is the energy differences in the metal centered (MC) states. These states are non emissive as they undergo fast radiationless deactivations to the ground state. For these complexes, the magnitude of splitting between the two sets of orbitals is strongly dependent on the principle quantum number $n$. For instance, in case of Fe(II) complexes which involve $3 \mathrm{~d}$ orbitals, the splitting is small enough so that lowest excited state in $\mathrm{Fe}(\mathrm{II})$ polyimine complexes is $\mathrm{MC}$ in nature. ${ }^{34-35} \mathrm{On}$ the contrary, for the $\operatorname{Ir}(\mathrm{III})$ (5d orbitals) and $\mathrm{Ru}(\mathrm{II})$ complexes (4d orbitals), the ligand field splitting is high enough to push the MC states higher in energy and consequently, the lowest excited states in these complexes are metal to ligand charge transfer (MLCT) states. This is schematically represented in figure 1.8. However, for $\mathrm{Ru}(\mathrm{II})$ complexes the splitting is intermediate between that of $\mathrm{Fe}(\mathrm{II})$ and $\operatorname{Ir}(\mathrm{III})$ and the MC states are still thermally accessible. ${ }^{36}$ 


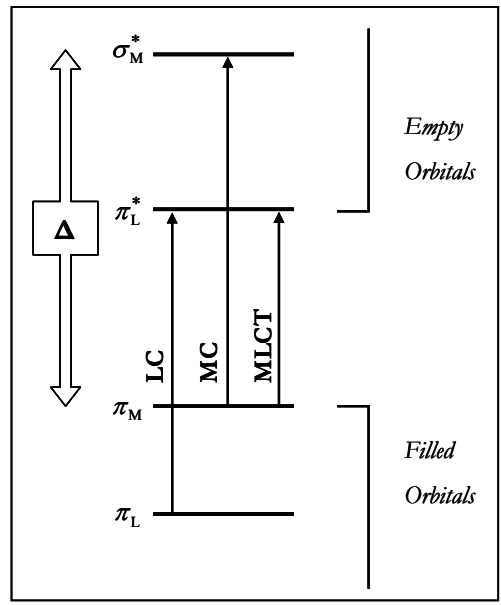

$\operatorname{Ir}(\mathrm{ppy})_{3}$

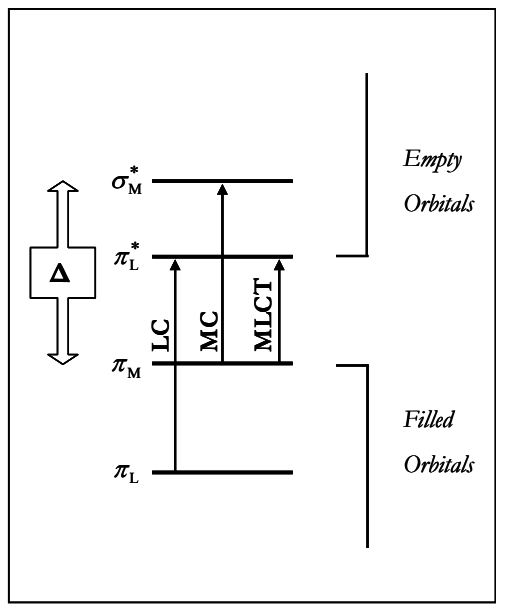

$\left[\mathrm{Ru}(\mathrm{bpy})_{3}\right]^{2+}$

Figure 1.8: Simplified energy level diagram representing different electronic transitions in prototype $\operatorname{Ir}(\mathrm{ppy})_{3}$ and $\mathrm{Ru}(\mathrm{bpy})_{3}$ complexes. The lowest excited states in both these complexes are MLCT. The crystal field splitting parameter, $\Delta$, is indicated on the left of each panel.

As can be seen from fig 1.8 the two complexes $\operatorname{Ir}(\mathrm{ppy})_{3}$ and $\operatorname{Ru}(\mathrm{bpy})_{3}$ have their lowest excited states as MLCT in character. However, due to different extent of splitting, the MLCT energies are different. For instance in case of $\operatorname{Ir}(\text { ppy })_{3}$, the MLCT absorption is at $375 \mathrm{~nm},{ }^{37}$ whereas for $\mathrm{Ru}(\mathrm{bpy})_{3},{ }^{36,38}$ it is at $450 \mathrm{~nm}$. In these complexes, the lowest excited ${ }^{1} \mathrm{MLCT}$ state undergoes an ISC to the ${ }^{3}$ MLCT state from which the emission occurs. Since the emitting states are charge transfer in nature, they are solvent dependent. In other words, the extent of stabilization due to solvent molecules plays a key role in determining the emission energies. The emissions from the triplet MLCT states are broad and structureless due to the stronger displacement of the excited state nuclear coordinate relative to the ground state. These states are long lived $(1-2 \mu \mathrm{s})$ in deaerated conditions. In this thesis, $\operatorname{Ir}(\mathrm{III})$ and $\mathrm{Ru}$ (II) complexes with amine functionality and a $\mathrm{Ru}(\mathrm{II})$ complex with tripodal geometry with thiolated terminal groups have been explored. Nanoassemblies with CdTe and $\mathrm{CdSe} / \mathrm{ZnS}$ NQDs have been fabricated with the abovementioned complexes. Photoinduced energy and electron transfer has been studied in these nanoassemblies. 


\section{Section 1c - Photoinduced processes}

Different deactivation pathways for the complexes and quantum dots have been explored in the previous sections. However, in the nanoassemblies, where metal complexes are bound to the quantum dots, a variety of photoinduced processes are possible. For instance, the excitation energy of the quantum dots could be transferred to the metal complexes nonradiatively, through an energy transfer process, thereby quenching the emission of the dots. On the other hand, quantum dots could be sensitized in a similar manner wherein the excited state of the metal complex is deactivated via energy transfer. Deactivation of the excited states could also be accompanied via a charge transfer mechanism. In this chapter, brief descriptions of these different photoinduced processes that are encountered in this thesis are presented.

\section{Energy transfer}

An excited molecule can undergo relaxation to ground state by transferring its excitation energy to another species with lower excited state, thereby exciting the acceptor molecule. This can be represented as

$$
D *+A \rightarrow D+A *
$$

where D and A are the energy donor and acceptor respectively and * represents the excited state.

Through certain interactions, $\mathrm{D}^{*}$ could undergo a vertical transition to $\mathrm{D}$ simultaneously with the jump of $A$ to $A^{*}$. In other words, the electronic transition moment which corresponds to $D^{*} \rightarrow D$ can trigger the $A \rightarrow A^{*}$ transition. Different mechanisms that cause these transitions are explained below.

\section{Radiative or 'trivial' energy transfer}

In a simple mechanism, the energy dissipated by the donor molecule could be reabsorbed by the acceptor molecule in a radiative process. ${ }^{39}$ The mechanism involved in this process can be understood on the basis of simple optical principles of absorption and emission. The acceptor molecule simply intercepts 
the donor emission, but does not influence in any way the emission ability of the donor. This process is favorable when the acceptor concentration is high in the path of emitted photon and the emission of the acceptor has a good spectral overlap with the acceptor absorption. However, this process could be distinguished from the energy transfer through other mechanisms by monitoring the donor excited state lifetimes. Unlike other mechanisms (nonradiative), the donor lifetime is not shortened as the acceptor molecule does not influence the emission capability of the donor.

\section{Förster resonance energy transfer (FRET) $)^{1-2,39-42}$}

When the donor molecule is excited, the excited state, $\mathrm{D}^{*}$ consists of an electron in the LUMO. This electronically excited molecule is assumed to behave like a classical oscillating dipole. This oscillating charge causes electrostatic forces to be exerted on the neighboring molecules. The oscillating dipole induces a perturbation on the electron in the ground state of the acceptor molecule, A, in such a way that the electron in A can oscillate with the same frequency as electron in $D^{*}$. In other words, a resonance condition is set up between the $\mathrm{D}^{*}$ and $\mathrm{A}$ fragments. This interaction is analogous to the effect of the electric field vector of light on molecules. This 'resonance' interaction causes the formation of $\mathrm{A}^{*}$ with the simultaneous deactivation of $\mathrm{D}^{*}$. This process is schematically represented in figure 1.9.

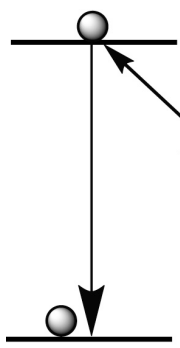

$\mathrm{D}$

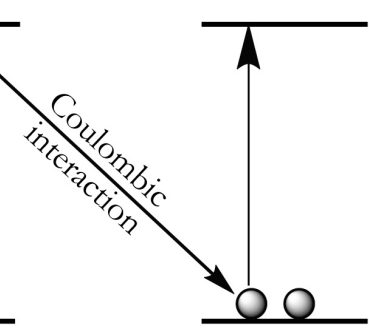

A

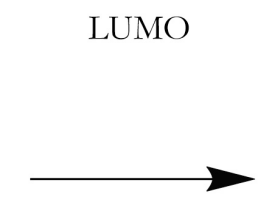

HOMO

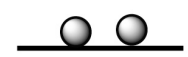

$\mathrm{D}$

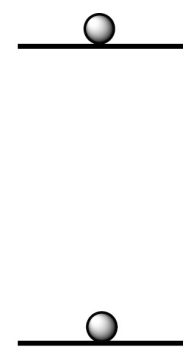

A

Figure 1.9: Schematic representation of resonance energy transfer through coulombic interactions. 
The theory of this resonance energy transfer was developed by Theodore Förster, a German physicist. Hence this mechanism is called Förster resonance energy transfer (FRET). According to Förster theory, the conditions necessary for the photoinduced energy transfer are (i) a good spectral overlap between the donor and acceptor, (ii) large radiative rate constant for the donor, (iii) large molar extinction coefficient of the acceptor and (iv) small donor acceptor separation and (v) the appropriate relative orientation between their electronic transition dipoles. The energy transfer rate is given by

$$
k_{T}=\frac{Q_{D} \kappa^{2}}{\tau_{D} r^{6}}\left(\frac{9000(\ln 10)}{128 \pi^{5} N n^{4}}\right) \int_{0}^{\infty} F_{D}(\lambda) \varepsilon_{A}(\lambda) \lambda^{4} d \lambda
$$

where $Q_{D}$ is the quantum yield of the donor, $\kappa^{2}$ is the orientation factor which is dependent on the angle between the donor and the acceptor electronic transition dipole moments, $\tau_{D}$ is the lifetime of the donor in absence of the acceptor, $\mathrm{r}$ is the donor-acceptor separation, $\mathrm{n}$ is the refractive index of the environment. $F_{D}(\lambda)$ is the corrected fluorescence intensity of the donor in the wavelength range $\lambda$ to $\lambda+\Delta \lambda$, with total intensity normalized to unity. $\varepsilon_{A}(\lambda)$ is the molar extinction coefficient of the acceptor at $\lambda$. The integral term in the equation represents the resonance condition, which is the spectral overlap integral given by the following equation

$$
J(\lambda)=\int_{0}^{\infty} F_{D}(\lambda) \varepsilon_{A}(\lambda) \lambda^{4} d \lambda \quad 1.2
$$

A more useful term that can be derived from equation 1.1 is the Förster distance, $R_{0}$, which is the distance between the donor and the acceptor when the energy transfer is $50 \%$ efficient. This is given by equation 1.3

$$
R_{0}^{6}=\frac{9000(\ln 10) \kappa^{2} Q_{D}}{128 \pi^{5} N n^{4}} \int_{0}^{\infty} F_{D}(\lambda) \varepsilon_{A}(\lambda) \lambda^{4} d \lambda \quad 1.3
$$

The energy transfer rate is inversely proportional to the $6^{\text {th }}$ power of the donor-acceptor separation. Hence the D-A separation drastically influences the 
energy transfer through Förster mechanism. Using equation 1.3 in 1.1 and simplifying would yield

$$
k_{T}(r)=\frac{1}{\tau_{D}}\left(\frac{R_{0}}{r}\right)^{6} \quad 1.4
$$

In the above equation all the parameters except $r$, the donor-acceptor separation can be experimentally measured. In order to calculate the donoracceptor separation, it is important to know the efficiency of the energy transfer.

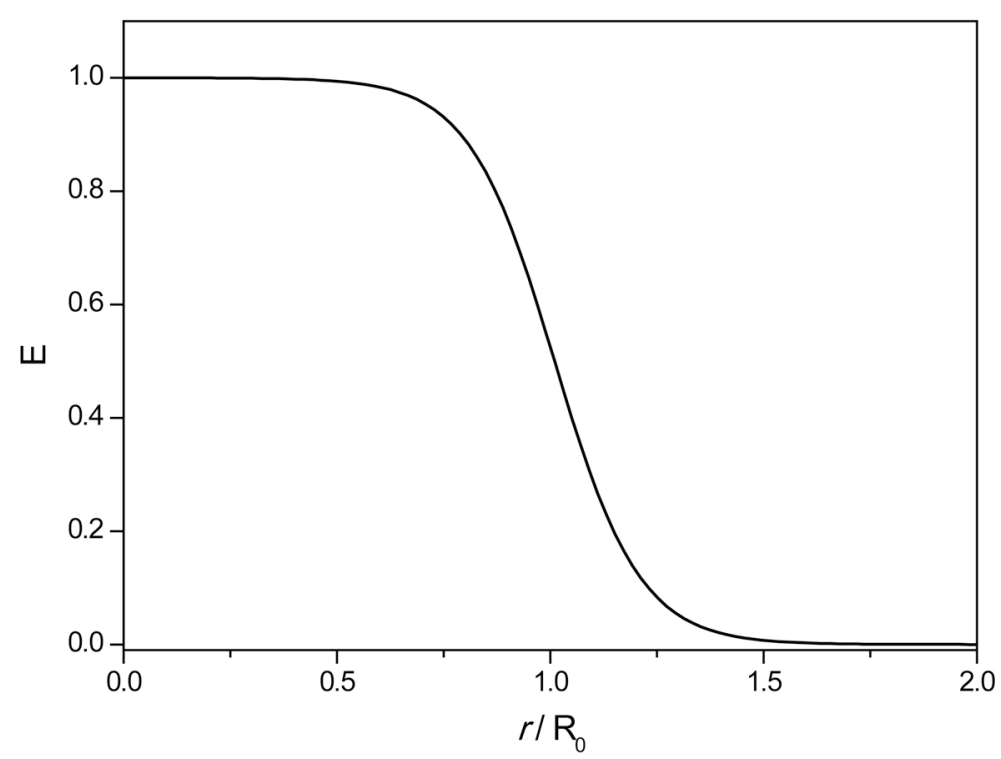

Figure 1.10: Dependence of energy transfer efficiency, E on distance. $\mathrm{R}_{0}$ is the Förster radius.

The FRET efficiency could be calculated either by the quenching of the donor or the enhancement of the acceptor emission. The most common and easiest method is the former one. The quenching efficiency is thus given by 


$$
E=1-\frac{\tau}{\tau_{0}} \quad 1.5
$$

where $\tau$ and $\tau_{0}$ are the excited state lifetimes of the donor molecule in absence and in presence of the acceptor molecule respectively. The energy transfer efficiency has a sigmoidal dependence on the ratio of $r / R_{0}$ and is represented by the curve in figure 1.10. From this curve the donor-acceptor separation can be calculated and hence the energy transfer rate is computed using equation 1.4 .

\section{Dexter energy transfer ${ }^{2,39,43}$}

When the electron cloud in the LUMO of $\mathrm{D}^{*}$ overlaps with the electron cloud of $\mathrm{A}$, the perturbation induced by $\mathrm{D}^{*}$ on $\mathrm{A}$ could result in an energy transfer that is mediated through a charge transfer process. This process unlike FRET must require collision of donor and acceptor. While the FRET mechanism has a classical oscillating dipole analogy, the energy transfer through exchange interactions is purely quantum mechanical in nature. Schematic representation of energy transfer through exchange mechanism is represented in figure 1.11.

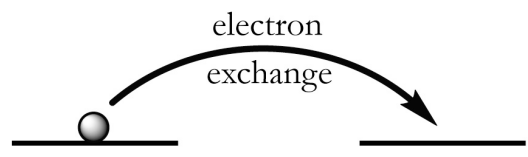

LUMO
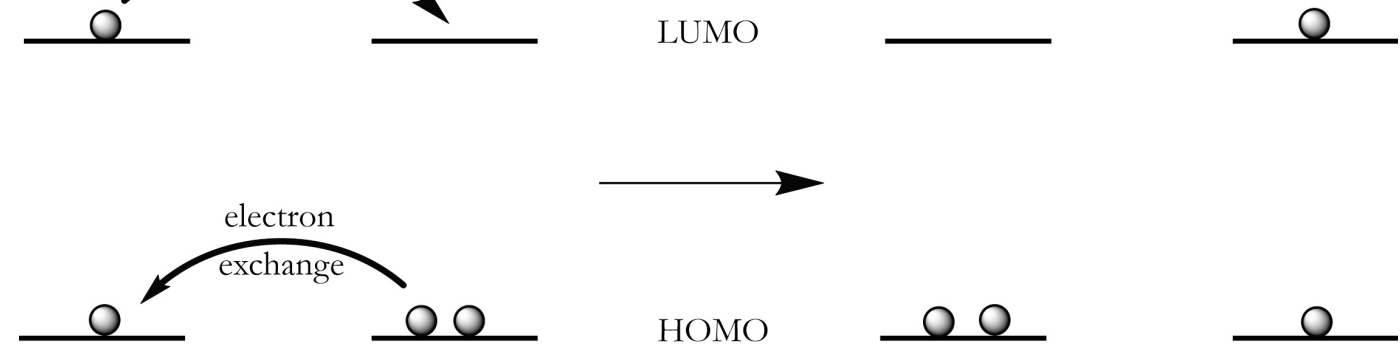

HOMO

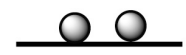

$\mathrm{D}$

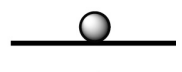

A

Figure 1.11: Schematic representation of energy transfer through exchange interactions. 
The theory for this process was worked out by D. L. Dexter and hence this process is known as Dexter energy transfer. Dexter energy transfer will be favorable if there are collisions between donor and acceptor that would result in overlap of orbitals. The energy transfer rate is given by

$$
k_{T}=K J \exp \left(-\frac{2 R_{D A}}{L}\right) \quad 1.6
$$

where $K$ is related to the specific orbital interactions, $J$ is the spectral overlap integral normalized to the extinction coefficient of the acceptor similarly as in the Förster theory and $R_{D A}$ is the donor-acceptor separation relative to their van der Waals radii $\mathrm{L}$. The dependence of the donor-acceptor separation on the energy transfer rate is more drastic than in the case of FRET as can be seen from equation 1.6. The distance between the donor and acceptor in this process usually falls between 5 - $10 \AA$. Since the Dexter mechanism involves charge transfer processes, the energy transfer rate is strongly dictated by the spin multiplicity of the excited state of the donor and the ground state of the acceptor. Spin allowed transitions usually give rise to a very efficient energy transfer whereas those involving spin forbidden transitions are inefficient.

\section{Photoinduced electron transfer}

Photoinduced electron transfer is one of the most important classes of photochemical reactions that occur in nature. This process involves deactivation of an excited molecule, accompanied by a charge transfer to another species (or different fragment of same species). The electron transfer can be inter or intramolecular in nature. Schematically these two processes are represented in figure 1.12 . 


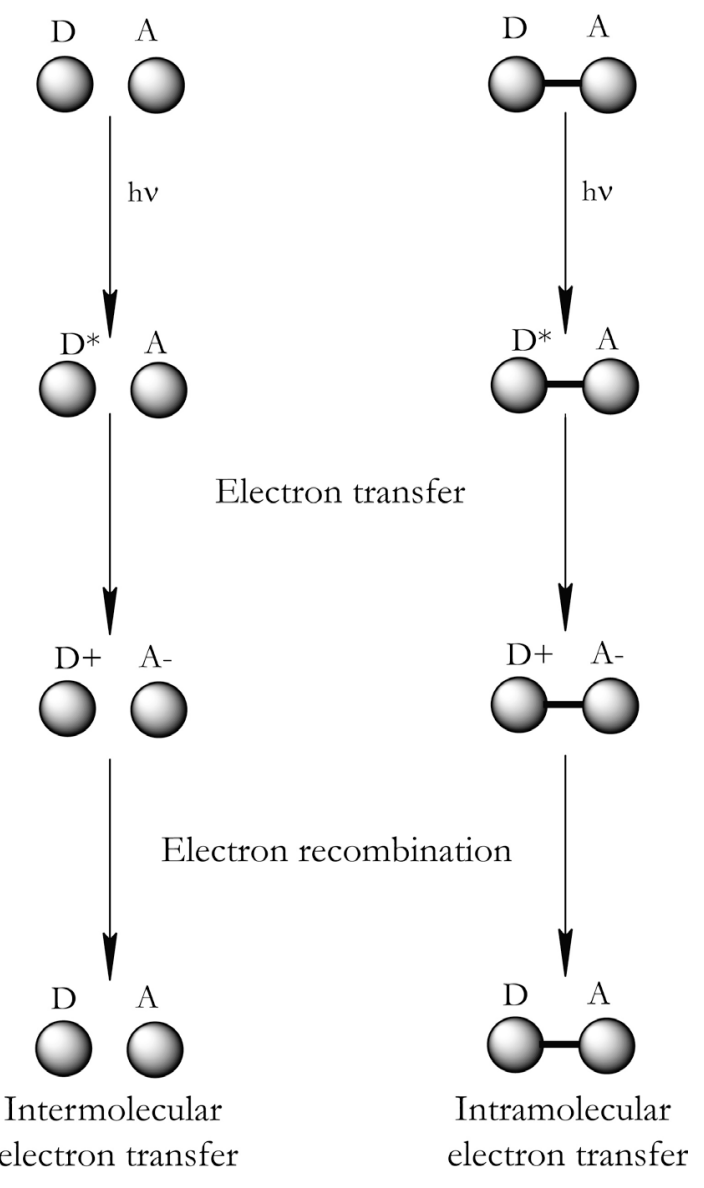

Figure 1.12: Schematic representation of inter and intramolecular electron transfer mechanisms.

Following Franck-Condon excitation, the electron transfer takes place giving rise to a charge separated state followed by charge recombination to the ground state. It is possible to determine from electrochemical and photophysical properties of the two species involved whether the process is thermodynamically feasible. The Gibb's free energy change that accompanies such a photoinduced charge transfer reaction is given by Rehm-Weller equation. ${ }^{44}$

$$
\Delta G_{e T}=E_{o x}-E_{\text {red }}-E_{00}-w(r)
$$


where $E_{o x}$ is the first oxidation potential of the electron donor, $E_{r e d}$ is the first reduction potential of the acceptor, $E_{00}$ is the energy of the $0-0$ transition of the moiety that is excited which is determined by the emission measurements at $77 \mathrm{~K}$ and $w(r)$ is the work term arising from the Coulombic interactions between the charges. The work term is given by

$$
w(r)=\frac{e^{2}}{4 \pi \varepsilon_{0} \varepsilon_{S} R_{D A}}+e^{2} / 8 \pi \varepsilon_{0}\left(\frac{1}{r^{+}}+\frac{1}{r^{-}}\right)\left(\frac{1}{\varepsilon_{s e}}-\frac{1}{\varepsilon_{s}}\right)
$$

where $e$ is the elementary charge, $\varepsilon_{0}$ is the vacuum permittivity constant, $\varepsilon_{s}$ is the dielectric constant of the solvent, $\varepsilon_{s e}$ is the dielectric constant of the solvent in which the electrochemistry is measured, $R_{D A}$ is the separation between the electron donor and acceptor, $r^{+}$and $r$ are the effective ionic radii of the donor and acceptor cation and anion respectively. For electron transfer to be thermodynamically favorable, the Gibb's free energy, $\Delta G_{\mathrm{eT}}$ should be negative.

The theory behind electron transfer was put forward by Rudolph Marcus and Noel Hush. ${ }^{45}$ According to this theory, after the formation of the first excited state, $\mathrm{D}^{*}$-A (or $\mathrm{D}^{*}$ in case of intramolecular electron transfer), reorganization of the nuclear geometry of this state occurs over time with respect to the surrounding environment. This reorganization takes place till a point where the energy of the reorganized state is equal to the charge separated state $\left(\mathrm{D}^{+}-\mathrm{A}^{-}\right)$. Thus solvent reorganization energy plays a very key role in the electron transfer reactions. The electron transfer rate as described by Marcus theory is given by

$$
k_{e T}=\kappa_{e l} v_{n} \exp \left(-\frac{\Delta G^{\#}}{k_{B} T}\right)
$$

where $\kappa_{e l}$ is the electronic transmission coefficient, which is the probability of the excited state converting to charge transfer state at the cross over point (the point of intersection of the potential energy surfaces of the excited state and the charge transfer state). $v_{n}$ is the nuclear factor which represents the frequency of passage through the transition state and $\Delta G^{\#}$ is the free energy of 
activation which is a function of overall free energy change and solvent reorganization energy. It is given by

$$
\Delta G^{\#}=\left(\Delta G_{0}+\lambda\right)^{2} / 4 \lambda
$$

The total reorganization energy is described as the potential energy change accompanying the nuclear reorganization in response to the electronic changes while going from the excited state to the charge separated state.

\section{Scope of the thesis}

Nanoassemblies based on semiconductor nanocrystal quantum dots and metal complexes have potential applications in various optoelectronic devices like OLEDs, photovoltaics and sensors. These assemblies constitute two fragments with one having size dependent behavior (NQDs) and the other having excellent emission and charge transfer properties (metal complexes). From an applications point of view, the understanding of the photoinduced processes in these assemblies is of quintessential importance. This thesis work is an attempt to explore the possible photoinduced processes in such nanoassemblies.

In chapter 3, two metal complexes with terminal amino groups have been used in conjunction with $\mathrm{CdTe}$ and $\mathrm{CdSe} / \mathrm{ZnS}$ quantum dots. Ir(III) complexes with CdTe quantum dots have been employed to demonstrate a photoinduced energy transfer from the metal complex to the NQDs. Ru(II) complexes with amino terminal group have been anchored onto the surface of $\mathrm{CdSe} / \mathrm{ZnS}$ through a coordinative ligand exchange strategy. In this case, the NQDs sensitize the metal complex. The former strategy is interesting due to potential applications in fabricating OLEDs, and the latter serves as a useful model for light harvesting systems.

Chapter 4 explores $\mathrm{Ru}(\mathrm{II})$ complexes with more complicated geometry. Specifically, tripodal $\mathrm{Ru}(\mathrm{II})$ complexes with terminal thiol and tert-butly groups have been investigated. The aim of this chapter is to demonstrate the versatility 
of such a redox active molecule and the stability of the tripodal structure for binding to different surfaces. Self assembled monolayers on ultrathin bulk gold substrates have been fabricated. Photophysics and electrochemistry of the tripodal molecule in solution and on surface have been carried out. To demonstrate further applicability of such a system in molecular electronics, a junction based on the SAM of Ru-tripod between two electrodes (eutectic GaIn and gold) has been constructed. Rectification in such metal-moleculemetal junction has been shown.

Chapter 5 deals with the nanoassemblies based on Ru-tripods and green emitting CdTe quantum dots. Ru-tripods with (alkyl thiolate, Ru-SAc) and without (tertiary butyl. Ru-tert-Bu) anchoring groups have been employed in order to quantify the photoinduced processes in the assembly. Upon exciting the CdTe NQD, there is an electron transfer from the CB edge of CdTe to the $\mathrm{Ru}(\mathrm{II})$ moieties. In case of the CdTe with Ru-SAc, the hot carrier relaxation is suppressed by an energy transfer to the $\mathrm{Ru}(\mathrm{II})$ fragment followed by an electron transfer to Ru-SAc.

The electron transfer process occurs on a time scale of $400 \mathrm{ps}$ and the recombination takes place in $325 \mathrm{~ns}$. Even in the reference compound, Ru-tert$\mathrm{Bu}$, electron transfer is demonstrated; however, due to lack of any strong binding interactions, suppression of hot carrier relaxation through energy transfer is not evident. Such charge transfer processes is of interest due to the potential applications in hybrid solar cells.

Extending the nanoconjugates to inorganic-organic systems, zeolite L crystals loaded with small organic dye molecules have been functionalized with NQDs on the surface of the zeolites. Chapter 6 deals with the FRET in such QDzeolite nanoconjugates. Two different cationic dye molecules, thionine and oxonine have been employed. Yellow emitting CdSe/ZnS NQDs have been used to coat the outer surface of the zeolites. A vectorial energy transfer from outside the zeolite crystal to the dye molecules entrapped inside of its channels has been demonstrated. Such an artificial antenna mechanism in host guest systems is interesting from light harvesting perspectives. 


\section{References}

1. Lackowicz, J. R., In Principles of Fluorescence Spectroscopy, 3rd ed.; Springer 2006; pp 281.

2. Valeur, B., In Molecular Fluorescence: Principles and Applications, Wiley: Weinheim, 2001.

3. Goldstein, A. N.; Echer, C. M.; Alivisatos, A. P., Science 1992, 256, 1425.

4. Tolbert, S. H.; Alivisatos, A. P., Ann. Rev. Phys. Chem. 1995, 46, 595.

5. Vossmeyer, T.; Katsikas, L.; Giersig, M.; Popovic, I. G.; Diesner, K.; Chemseddine, A.; Eychmueller, A.; Weller, H., J. Phys. Chem. 2002, 98 , 7665.

6. Alivisatos, A. P., J. Phys. Chem. 1996, 100, 13226.

7. Ekimov, A. I.; Onushchenko, A. A., Sov. Phys.-Semicond. 1982, 16, 775.

8. Rossetti, R.; Nakahara, S.; Brus, L. E., J. Phys. Chem. 1983, 79, 1086.

9. Klimov, V. I., Semiconductor and Metal Nanocrystals. Synthesis and Electronic and Optical Properties. Marcel Dekker: New York, 2004; p 159

10. Ellert, C.; Schmidt, M.; Schmitt, C.; Reiners, T.; Haberland, H., Phys. Rev. Lett. 1995, 75, 1731.

11. Colvin, V. L.; Alivisatos, A. P.; Tobin, J. G., Phys. Rev. Lett. 1991, 66, 2786.

12. Kastner, M. A., Rev. Mod. Phys. 1992, 64, 489.

13. Efros, A. L.; Efros, A. L., Sov. Phys. Semicond. 1982, 16, 772.

14. Mittleman, D. M.; Schoenlein, R. W.; Shiang, J. J.; Colvin, V. L.; al., e., Phys. ReV. B: Condens. Matter 1994, 49, 14435.

15. Klimov, V. I., Ann. Rev. Phys. Chem. 2007, 58, 635.

16. Ekimov, A. I.; Hache, F.; Schanne-Klein, M. C.; Ricard, D.; Flytzanis, C.; Kudryavtsev, I. A.; Yazeva, T. V.; Rodina, A. V.; Efros, A. L., J. Opt. Soc. Am. B 1994, 11, 524.

17. Meijerink, A., Exciton dynamics and energy transfer processes in semiconductor nanocrystals. 2008; pp 277.

18. Klimov, V. I., J. Phys. Chem. B. 2000, 104, 6112.

19. Guyot-Sionnest, P.; Shim, M.; Matranga, C.; Hines, M., Physical Review B 1999, 60, R2181. 
20. Wang, H.; de Mello Donega, C.; Meijerink, A.; Glasbeek, M., J. Phys. Chem. B. 2005, 110, 733.

21. Klimov, V. I., Linear and nonlinear optical spectroscopy of semiconductor nanocrystals. In Handbook of Nanostructured Materials and Nanotechnology, Hari Singh, N.; M.Sc; Ph.D, Eds. Academic Press: Burlington, 2000; pp 451.

22. Kamat, P. V.; Dimitrijevic, N. M.; Nozik, A. J., The Journal of Physical Chemistry 1989, 93, 2873.

23. Guyot-Sionnest, P.; Wehrenberg, B.; Yu, D., J. Chem. Phys. 2005, 123, 074709.

24. Nirmal, M.; Brus, L., Acc. Chem. Res. 1999, 32, 407.

25. Dabbousi, B. O.; Rodriguez-Viejo, J.; Mikulec, F. V.; Heine, J. R.; Mattoussi, H.; Ober, R.; Jensen, K. F.; Bawendi, M. G., J. Phys. Chem. B 1997, 101, 9463.

26. Hines, M. A.; Guyot-Sionnest, P., J. Phys. Chem. 1996, $100,468$.

27. Nazzal, A. Y.; Qu, L.; Peng, X.; Xiao, M., Nano Letters 2003, 3, 819.

28. Xia, Y.; Zhang, T.; Diao, X.; Zhu, C., Chem. Lett. 2007, 36, 242.

29. Murray, C. B.; Kagan, C. R.; Bawendi, M. G., Annu. Rev. Mater. Sci. 2000, 545 .

30. Murray, C. B.; Norris, D. J.; Bawendi, M. G., J. Am. Chem. Soc. 1993, 115, 8706.

31. Pearson, R. G., J. Am. Chem. Soc. 1963, 85, 3533.

32. Montalti, M.; Credi, A.; Prodi, L.; Gandolfi, M. T., Handbook of Photochemistry. 3rd ed.; Taylor \& Francis: 2006.

33. Roundhill, D. M., Photochemistry and Photophysics of Metal Complexes. Plenum Press: New York, 1994.

34. Braterman, P. S.; Song, J. I.; Peacock, R. D., Inorg. Chem. 2002, 31, 555.

35. Hamann, C.; Zelewsky, A. v.; Neels, A.; Stoeckli-Evans, H., Dalton Trans. 2004, 402.

36. Jurius, A.; Balzani, V.; Barigelletti, F.; Campagna, S.; Belser, P.; von Zelewsky, A., Coord. Chem. Rev. 1988, 84, 85.

37. Flamigni, L.; Barbieri, A.; Sabatini, C.; Ventura, B.; Barigelletti, F., Top. Curr. Chem. 2007, 281, 143. 
38. Campagna, S.; Puntoriero, F.; Nastasi, F.; Bergamini, G.; Balzani, V., Top. Curr. Chem. 2007, 280, 117.

39. Turro, N., In Modern Molecular Photochemistry, University Science Books: Sausalito, 1991.

40. Förster, T., Ann. Physik 1948, 2, 55.

41. Förster, T., In Fluorezenz. Organischer Verbindungen, Vandenhoech \& Ruprech: Göttingen, 1951.

42. Förster, T., Disc. Faraday Soc. 1959, $27,7$.

43. Dexter, D. L., J. Chem. Phys. 1953, 21, 836.

44. Rehm, D.; Weller, A., Isr. J. Chem 1970, 8, 259.

45. Marcus, R. A., J. Chem. Phys. 1956, 24, 966. 


\title{
CHAPTER 2
}

EXPERIMENTAL TECHNIQUES

\begin{abstract}
In this chapter, the major experimental methods used in the thesis are described. Steady-state and time resolved absorption and emission techniques used for the photophysics of the nanoassemblies are described here. In addition, techniques used for surface characterization like atomic force microscopy, time resolved confocal microscopy and general electrochemical techniques are outlined as well.
\end{abstract}




\section{Steady State Measurements ${ }^{1}$}

\section{Absorption Measurements}

Absorbance $(A)$ is defined as $A_{\lambda}=-\log _{10}\left(I / I_{0}\right)$, with $I$ as the intensity of light at the specified wavelength that has passed through a sample. $I_{0}$ is the intensity of the incident light before passing through the sample; absorbance therefore is a dimensionless quantity, which refers to the amount of transmitted light over incident light. ${ }^{1}$ In the absence of other phenomena, like scattering, aggregation of the sample or diffraction, the absorbance follows the Lambert-Beer law (see equation 2.1). The Lambert-Beer law states that the absorbance of a sample is proportional to the path of light through the medium $(l$ in $\mathrm{cm})$, to the molar absorption coefficient $\left(\varepsilon\right.$ in $\left.\mathrm{L}_{\mathrm{mol}}{ }^{-1} \cdot \mathrm{cm}^{-1}\right)$, a characteristic value for chromophores, and to the concentration of the sample $\left(c\right.$ in $\left.\mathrm{mol} \mathrm{L}^{-1}\right)$.

$$
A_{\lambda}=-\log _{10}\left(I / I_{0}\right)=\varepsilon \cdot l \cdot c
$$

Figure 2.1 shows a general setup for a spectrophotometer. The monochromator resolves the incident polychromatic light into different wavelengths by a diffraction grating. This incident monochromatic light is then guided to a beam splitter, where the light passes through a reference channel and the sample channel. This double-beam setup corrects for lamp intensitychanges during the measurement. Recording the absorption of the solvent in quartz cuvettes in the reference and sample chamber allows baseline correction instantaneously during the measurement. In this way, solvent absorption as well as differences between the two different light pathways are compensated. Absorption spectra shown in this thesis were recorded on a Varian Cary 5000 double-beam UV/Vis-NIR spectrophotometer. All spectra were recorded in quartz cuvettes $(1 \mathrm{~cm}$, Hellma), which for the oxygen free measurements have been modified for the freeze-pump-thaw technique (Figure 2.2) such that the cuvette can be connected to high vacuum. Low temperature emission spectra for glasses were recorded in $5 \mathrm{~mm}$ diameter quartz tubes that were placed in a liquid nitrogen Dewar equipped with quartz walls. 


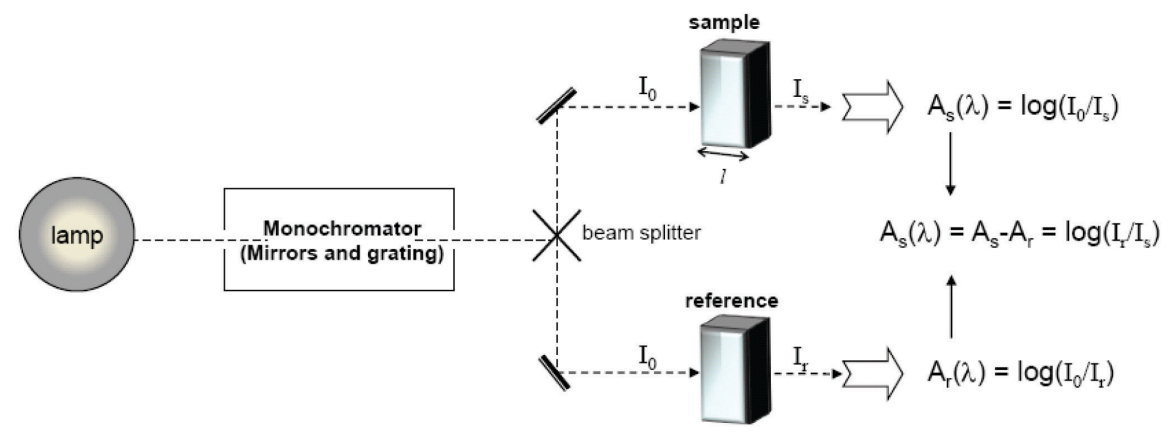

Figure 2.1 Schematic representation of a spectrophotometer.

Figure 2.2 a) shows a homemade vacuum cuvette, which has been built from a quartz cuvette (4) that is connected by a quartz tube to a glass assembly comprising a sample compartment (3) for the freeze-pump-thaw procedure, a Teflon stopper (1) and a joint for the high vacuum line (2). The solution is added into the sample compartment (3) and before evacuation of the compartment it is frozen with liquid nitrogen avoiding solvent evaporation. Then, the Teflon stopper is closed and upon warming of the solution to room temperature, the oxygen dissolved in the liquid phase, equilibrates into the vacuum (Figure $2.2 \mathrm{~b}$ ). This procedure is repeated until the vacuum in the cuvette reaches approximately $10^{-6}$ bar and most of the oxygen is removed. 


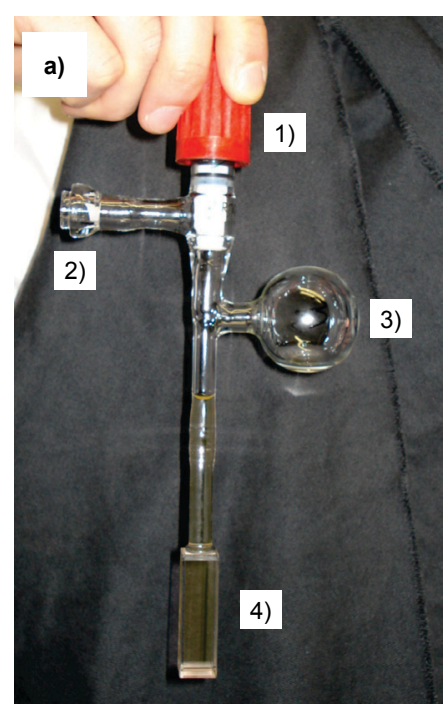

b)
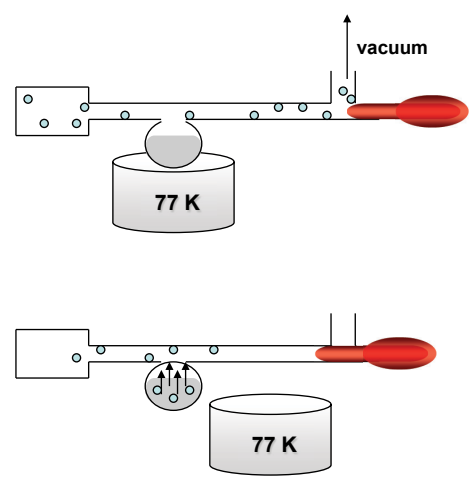

Figure 2.2 a) Photograph of the homemade vacuum cuvette, b) Schematic representation of the freeze-pump-thaw technique.

\section{Emission and Excitation Measurements}

\section{Emission Measurements}

Emission spectra are measured using spectrofluorometers and reveal the wavelength distribution of the emission upon excitation at a certain single wavelength. The spectra can be presented using for the $\mathrm{x}$-axis a wavelength $(\lambda$, $\mathrm{nm})$ or a wave-number scale $\left(\tilde{v}=v / c, \mathrm{~cm}^{-1}\right)$, both representing the photon energy (equation 2.2). However, for ease of understanding, all spectra in this thesis are shown plotting the $\mathrm{x}$-axis in a wavelength scale and the $\mathrm{y}$-axis in arbitrary units.

$$
E=h \cdot v=\frac{h \cdot c}{\lambda}
$$


Figure 2.3 shows a schematic diagram of a spectrophotometer used during this thesis. The excitation source is a xenon lamp, which has a high intensity between $200-800 \mathrm{~nm}$. Two monochromators are used, one between light source and sample chamber, the other between sample chamber and detector. Both monochromators are equipped with motors, to allow automatic scanning of wavelength. Photomultiplier tubes, placed at right angles to the excitation light beam, detect the luminescence and convert it into an electric signal. The spectrophotometer used in this thesis was equipped with two detectors, a UV/VIS- and NIR- detector. Between the first monochromator (seen from the lamp on) and the sample chamber, a beam splitter guides part of the excitation light to a reference cell, which contains a stable reference fluorophore and a reference detector, which detects the intensity from the standard solution and can thus monitor changes in the intensity of the xenon lamp.

Steady-state emission spectra in the spectral range of $300-800 \mathrm{~nm}$ were recorded on a HORIBA Jobin-Yvon IBH FL-322 Fluorolog 3 spectrometer equipped with a $450 \mathrm{~W}$ xenon arc lamp, double grating excitation and emission monochromators $(2.1 \mathrm{~nm} / \mathrm{mm}$ dispersion; 1200 grooves $/ \mathrm{mm})$ and a Hamamatsu R928 photomultiplier tube or a TBX-4-X single-photon-counting detector. Emission and excitation spectra were corrected for source intensity (lamp and grating) and emission spectral response (detector and grating) by standard correction curves. Emission spectra were corrected for excitation intensity (lamp and grating) and detector response (detector and grating) by standard correction curves. 


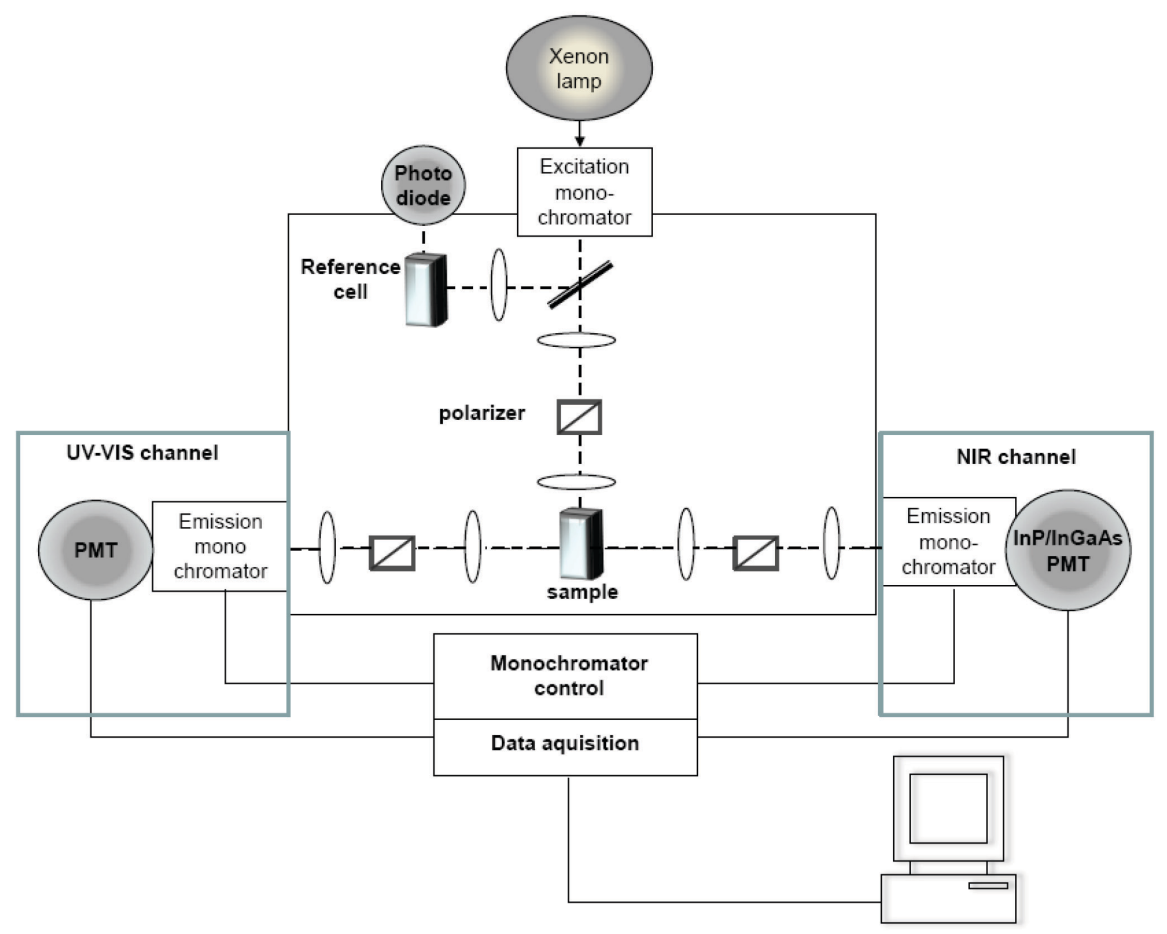

Figure 2.3 Schematic representation of a spectrophotometer equipped with two detector channels.

The fluorescence quantum yield $(\phi)$ represents the amount of photons emitted from a sample relative to the amount of photons absorbed. The quantum yield value (between zero and 1) is a measure to determine the probability for radiative decay processes. Quantum yields are measured in optically dilute solutions $(A(\lambda)<0.1)$ to avoid inner filter effects. One way to estimate the quantum yield of a fluorophore is by comparison with a standard compound of known quantum yield (equation 2.3). ${ }^{2}$ In general, the concentration of the sample and the standard compound are adjusted such that they are approximately of the same concentration at a stationary point of the absorption spectrum. The samples are excited at the stationary point and the integral of the emission bands are compared.

Equation 2.3 demonstrates the calculation of the quantum yield of the sample: 


$$
\phi_{x}=\phi_{r}\left[\frac{A_{r}\left(\lambda_{r}\right)}{A_{x}\left(\lambda_{x}\right)}\right]\left[\frac{I_{r}\left(\lambda_{r}\right)}{I_{x}\left(\lambda_{x}\right)}\right]\left[\frac{n_{x}^{2}}{n_{r}^{2}}\right]\left[\frac{D_{x}}{D_{r}}\right]
$$

where $A$ is the absorbance at the excitation wavelength $(\lambda), I$ is the intensity of the excitation light at the excitation wavelength $(\lambda), n$ is the refractive index of the solvent, $D$ is the integrated intensity of the luminescence and $\Phi$ is the quantum yield. The subscripts $r$ and $x$ refer to the reference and the sample, respectively. Since all quantum yields were performed at identical excitation wavelength for the sample and the reference, the term $\left(I_{r}\left(\lambda_{r}\right)\right) /\left(I_{x}\left(\lambda_{x}\right)\right)$ can be cancelled out. To reduce the error, the emission of the standard and the sample should occur at similar wavelength as well as the quantum yields should be on the same order of magnitude.

Quantum yields can also be measured by an absolute method with an integrating sphere (Figure 2.4.), which collects all the emitted photons with a calibrated photodiode and sets them into relation with the number of absorbed photons. Quantum yields were measured in some cases with a Hamamatsu Photonics absolute PL quantum yield measurement system (C9920-02, Figure 0.) equipped with a L9799-01 CW Xenon light source (150 W), monochromator, $\mathrm{C} 7473$ photonic multi-channel analyzer, integrating sphere and employing U6039-05 PLQY measurement software (Hamamatsu Photonics, Ltd., Shizuoka, Japan). 
a) Blank

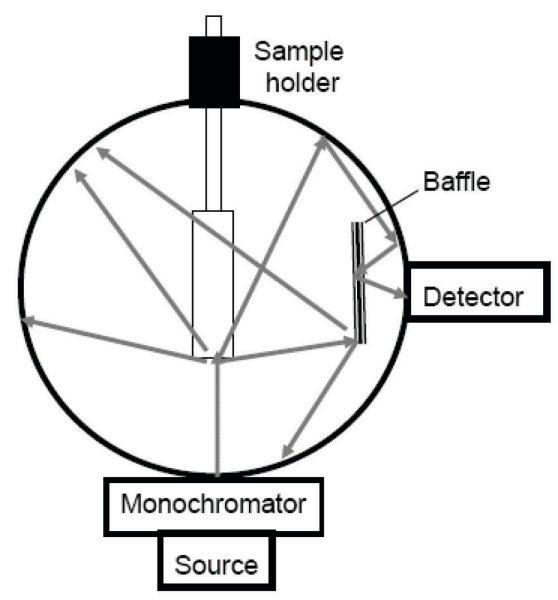

b) Sample

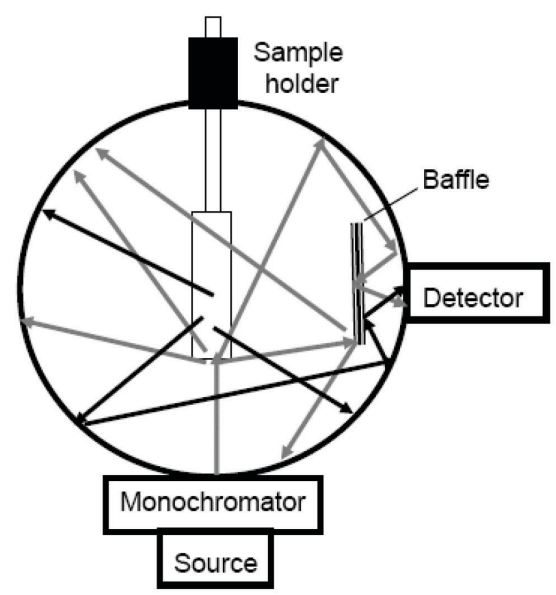

Figure 2.4 Schematic representation of the integrating-sphere quantum yield measurement. a) blank cuvette to detect the intensity of the excitation light, b) sample which absorbs and emits light.

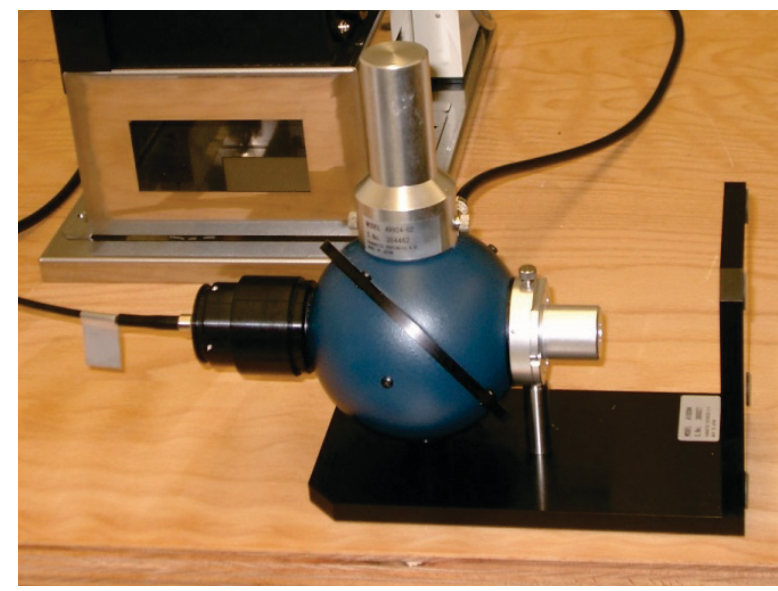

Figure 2.5 Integrating Sphere setup C9929-02. 


\section{Excitation Measurements}

Excitation measurements are converse to the emission measurements such that they display the dependence of emission intensity, measured at a single emission wavelength, from the excitation wavelength. Therefore, the emission monochromator is set to a fixed wavelength and the detector monitors the intensity changes upon variation of the excitation wavelength. Thus, the excitation spectra are corrected for the excitation monochromator grating and for the lamp intensity changes, which is realized using the reference channel R. Excitation spectra have been recorded using the same experimental setup as shown in Figure 2.3.

\section{Time-Resolved Spectroscopy}

Time-resolved spectroscopy measurements provide important insight into the excited states of molecules. For example, excited-state lifetimes can give information about the spin-multiplicity of the excited state, about the mechanism involved in various photoinduced processes, e.g. quenching, energy and electron transfer. Transient absorption spectroscopy helps us understanding the formation of the lowest-energy excited state of a system.

\section{Time-Resolved Emission Spectroscopy}

The lifetime of the excited state is defined by the time required for the emission intensity to fall to 1 /e of its initial value, which means until the concentration of excited states decreases to $1 / \mathrm{e}$ of its initial value. The lifetime is a statistical average value over many decay processes. In order to measure the excited-state lifetime, the sample is exposed to a pulse of light, where the pulse width is typically shorter than the decay time of the sample. The emission intensity decay time, which follows equation 2.4 , is recorded with a high-speed detection system.

$$
I(t)=I_{0} \cdot e^{-t / \tau}
$$


Sometimes multi-exponential decays are observed, due to different species in the solution, one species in different environments or an admixture of different excited states in the molecule. In these cases, the intensity decay is approximated as the sum of individual decays and is fitted multiexponentially: ${ }^{3}$

$$
I(t)=\sum_{i=1}^{n} \alpha_{i} e^{-t / \tau_{i}}
$$

with $\tau_{i}$ representing decay times, $\alpha_{i}$ the amplitudes of the components at $t=0$ and $n$ as the number of decay times.

The time-correlated single photon counting (TCSPC) setup reconstructs the time single photons need to reach the detector after the pulsed excitation of a sample. During the measurement a large number of excitation pulses are applied onto the sample. Each pulse gives an electrical signal to the start input of the time-to-amplitude (TAC) converter. When the sample emits, the first photon following the excitation pulse is recorded by the detector giving an electrical response, which is then directed as a stop signal to the TAC. The start pulse has initiated the charging of the capacitor while the stop pulse terminates it. Therefore, the final voltage is proportional to the time between start and stop pulses and is stored in a histogram (Figure 2.6). The x-axis in the histogram is the time difference between start and stop signal and the y-axis shows the number of photons detected for this time difference. The large number of repetitive measurements attains a good statistical fit for the time distribution of emitted photons. 


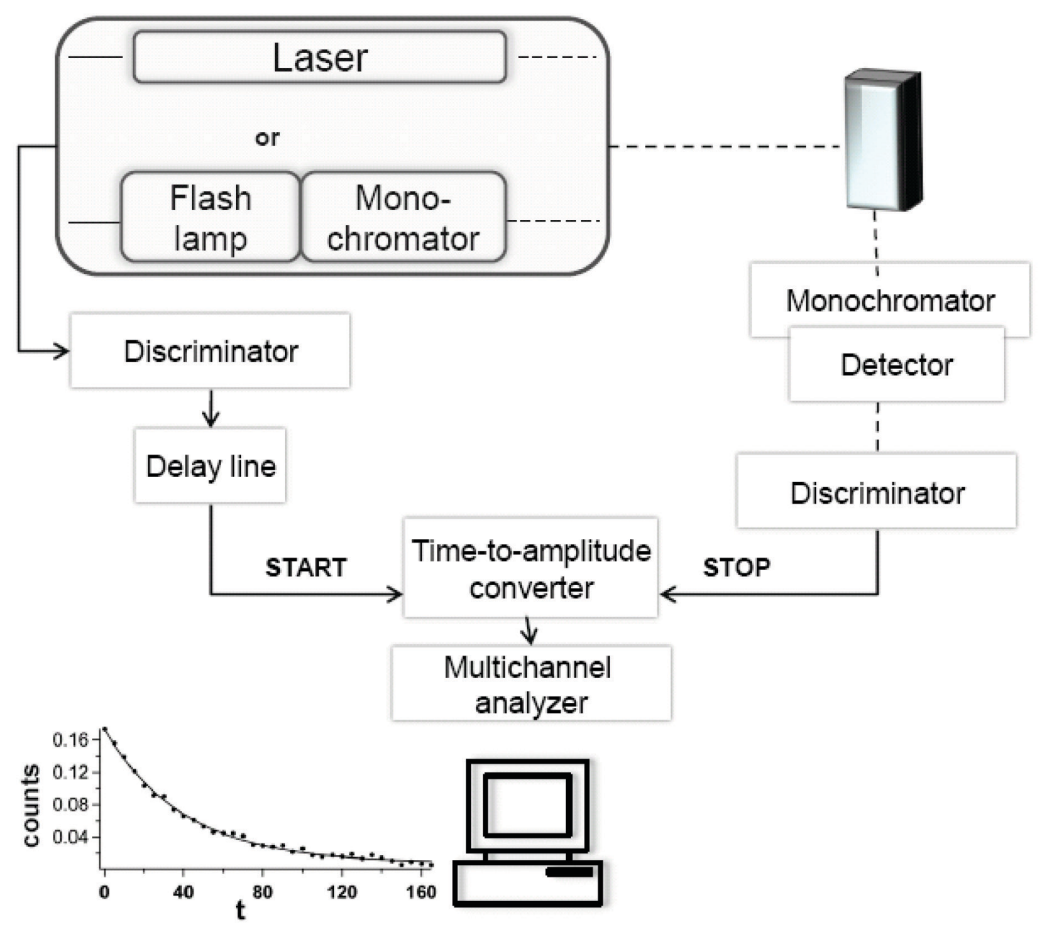

Figure 2.6 Schematic representation of the single photon counting setup.

Time-resolved measurements up to $\sim 5 \mu$ s were performed using the timecorrelated single-photon counting (TCSPC) option on the Fluorolog 3. Two NanoLEDs (402 nm; FWHM < 750 ps and 431 nm; FWHM $<200$ ps) with repetition rates between $10 \mathrm{kHz}$ and $1 \mathrm{MHz}$ were used to excite the samples. The excitation source was mounted directly on the sample chamber at $90^{\circ}$ to a double grating emission monochromator $(2.1 \mathrm{~nm} / \mathrm{mm}$ dispersion; 1200 grooves $/ \mathrm{mm}$ ) and collected by a TBX-4-X single-photon-counting detector. Signals were collected using an IBH DataStation Hub photon counting module and data analysis was performed using the commercially available DAS6 software (HORIBA Jobin Yvon IBH).

For excited-state lifetimes $>10 \mu$ s a different experimental setup was used, by equipping the Fluorolog 3 with the FL-1040 phosphorescence module with a 
$70-\mathrm{W}$ xenon flash tube (full-width at half maximum, FWHM $=3 \mathrm{~ms}$ ) with a variable flash rate $(0.05-25 \mathrm{~Hz})$. The signals were recorded on the TBX-4-X single-photon-counting detector and collected with a multichannel scaling (MCS) card in the IBH DataStation Hub photon-counting module and data analysis was performed as described above.

The multichannel analyzer consists of a multichannel card. The light input is discriminated for its intensity and if the input pulse amplitude falls within the threshold of the lower level discriminator and the upper level discriminator, the control and logic circuit is enabled and the peak amplitude is stored. In general, the signal intensities are collected in different channels for the duration of a dwell time after which an intensity counter moves to the next channel. After the acquisition time is reached, the collected total light intensities in all channels are counted and correlated to the time frame of each channel. Therefore, at the end of the measurement an intensity-time profile can be made.

The goodness of fit was assessed by minimizing the reduced chi squared function $\left(\chi^{2}\right)$ and visual inspection of the weighted residuals.

\section{Transient Absorption Spectroscopy ${ }^{4}$}

Time-resolved absorption spectroscopy is a powerful tool to obtain information about the nature of the excited state. As a differential method, transient absorption spectroscopy compares the electronic absorption spectra of the ground state and of the excited state at selected time delays after the excitation pulse. The absorption trace can be recorded over the full spectrum. Laser excitation promotes the molecules into an excited state, followed by measuring the absorption spectrum of that excited state by illuminating the sample with a pulsed white light xenon lamp at different time delays after the laser excitation. The delay between pump and probe pulse is electronically generated using a delay generator. The ground-state absorption spectrum is subtracted from the averaged recorded spectrum yielding the transient spectra. If the extinction coefficient of the excited state is higher than that of the ground state, we see a net increase in sample absorbance in the transient 
spectra, otherwise we see bleach. The spin-allowed ground-state absorption usually can have very high extinction coefficients, therefore, the extinction coefficient of the excited state can be much weaker and it can be difficult to obtain a $\Delta \mathrm{A}$ value intense enough. In these cases, it is advantageous to measure in an area different from the main absorption bands of the ground state. From the decay of the absorption bands, we can calculate the lifetime of the excited state. The timescale for transient absorption measurements can be in the nanosecond or sub picosecond timescale. For the NQDs measured in this work, it was found that the hot carrier relaxation was of the order of few hundred femtoseconds; while for the metal complexes, the general bleach of the lowest excited state (MLCT) was found to be of the order of $1 \mu$ s. During this work both nanosecond and femtosecond transient setups were used. 


\section{Overall Scheme}
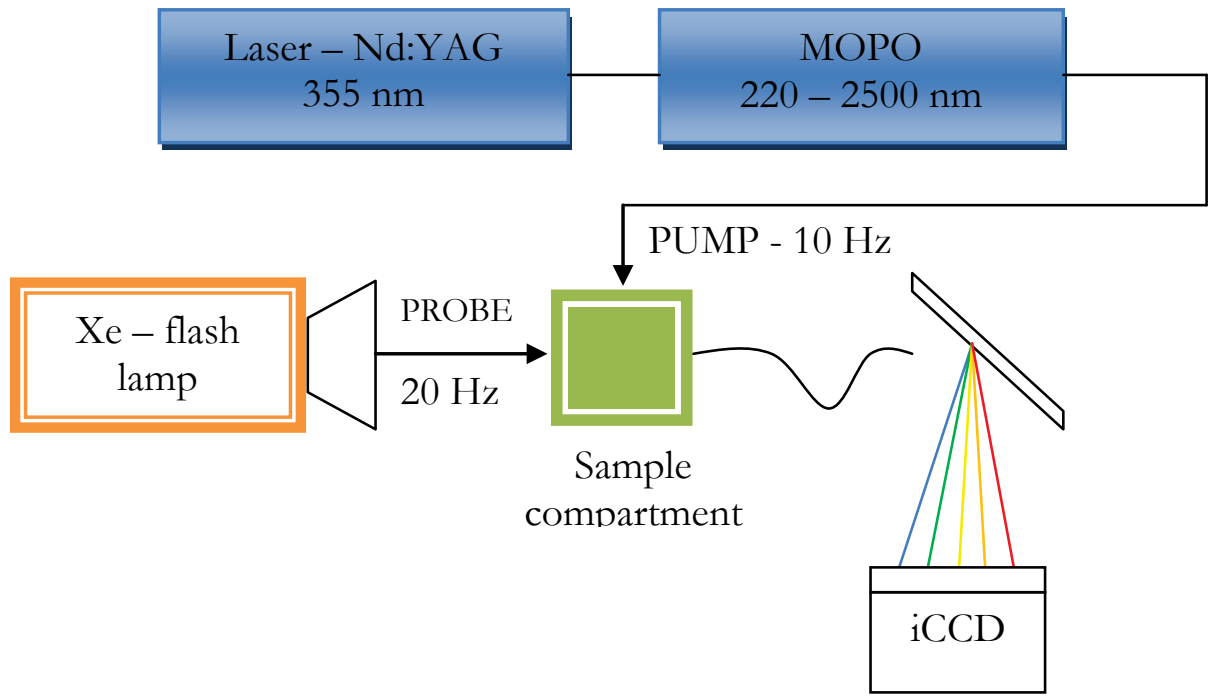

\section{Wiring Scheme}

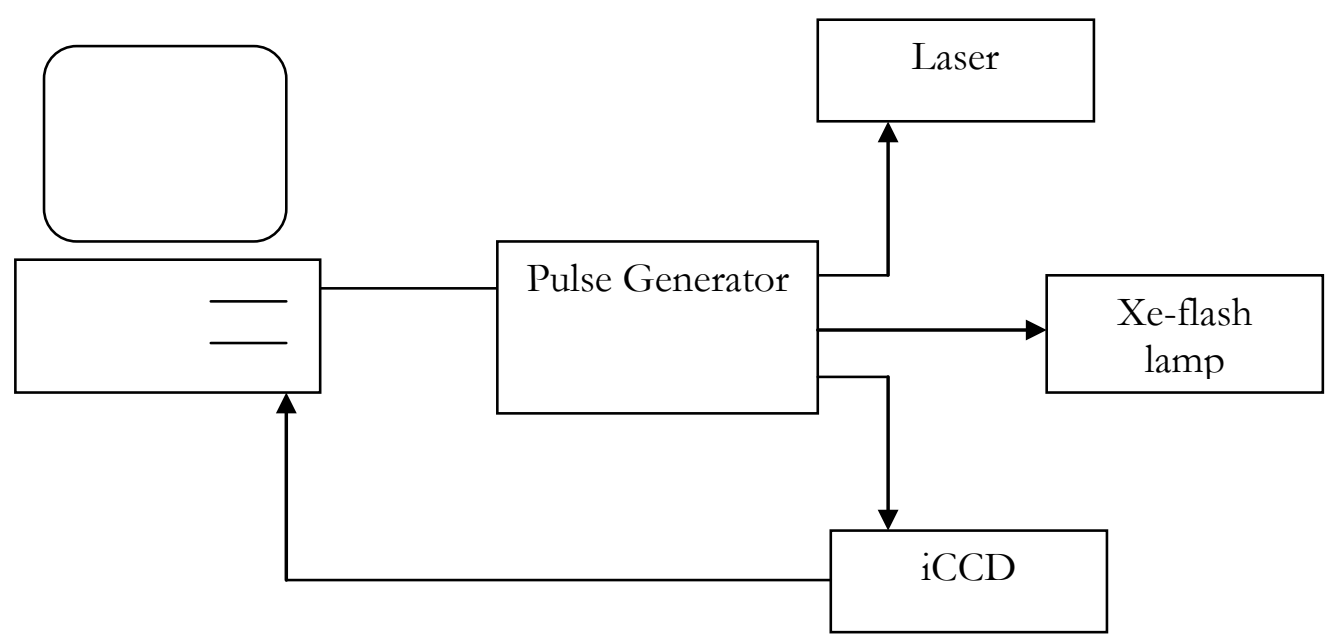

Figure 2.7. Schematic representation of a time-resolved nanosecond absorption setup. 
The detection line of the TA setup is shown in Figure 2.7, comprises a Xenon flash lamp (FWHM $10 \mu \mathrm{s}$ ) and a spectrograph (Acton SP-2156 with 150 $\mathrm{G} / \mathrm{mm}$ ) that fans out the light onto a gated 16 bit intensified CCD camera (PIMAX 1024 - UNIGEN) which is capable of recording 20 frames per second.

The sample is excited perpendicularly to the probing beam by an assembly of a frequency tripled Nd:YAG laser (Spectra Physics - Quanta Ray) which pumps a MOPO (mirrorless optical parametric oscillator - Spectra Physics MOPOSL) that can tune the wavelength in the range of $220-2500 \mathrm{~nm}$. A computercontrolled pulse generator (Berkley Nucleonics - BNC 565) times the lasing as well as the Xe-flash lamp and the camera. The whole system is controlled via an in-house developed LabView program. The transient absorption is calculated from the spectra recorded upon laser excitation and from the ground-state absorption taken between two laser pulses.

\section{Femtosecond transient absorption}

Sub-picosecond transient absorption setup is one of the most powerful techniques in studying the carrier dynamics in NQDs. For instance, the formation of 'hot carriers' and their relaxation to the band edge followed by subsequent bleach corresponding to the exciton recombination could be simultaneously monitored through pump induced absorption changes. In this thesis, such a setup was employed for the study of charge transfer between NQDs and $\mathrm{Ru}(\mathrm{II})$ complexes. The setup used for the sub-picosecond transient absorption measurements is shown in Figure 2.8. The laser system is based on a Spectra Physics Hurricane Ti-saphire regenerative amplifier system. This optical bench assembly of the Hurricane includes a seeding pump laser (Mai Tai), a pulse stretcher, a Ti-saphire regenerative amplifier, a Q-switched pump laser (Evolution) and a pulse compressor. The output power of the laser is typically $1 \mathrm{~mJ} /$ pulse (130 fs fwhm) at a repetition rate of $1 \mathrm{kHz}$. The pump probe setup employed a full spectrum setup based on two optical parametric amplifiers (Spectra-Physics OPA 800) as a pump (depending on the excitation wavelength) and a residual fundamental light $(150 \mu \mathrm{J} /$ pulse $)$ from the pump OPA was used for the generation of white light, which was detected with CCD 
spectrograph. The OPA was used to generate excitation pulses from $280-600$ $\mathrm{nm}$ (fourth harmonic signal of the OPA or idler). The white light generation was accomplished by focusing the fundamental $(800 \mathrm{~nm})$ into a stirred water cell equipped with barium bisfluoride or sapphire windows. The pump light was passed over a delay line (Physik Instrumente, M-531DD) that provided an experimental time window of $1.8 \mathrm{~ns}$ with the maxima resolution of $0.6 \mathrm{fs} / \mathrm{step}$. The energy of the probe pulses was $c a .5 \times 10^{-3} \mathrm{~mJ} /$ pusle. The angle between the pump and the probe beam was typically $7-10^{\circ}$. Samples were prepared in quartz cuvette $(1=0.1 \mathrm{~cm})$ to have an optical density of $c a .0 .8$ at the excitation wavelength. For the white light/CCD setup, the probe beam was coupled into a $400 \mu \mathrm{m}$ optical fiber after passing through the sample, and detected by a CDD spectrometer (Ocean Optics, PC2000). The chopper (Roffin Ltd., $f=10$ $-20 \mathrm{~Hz})$, place in the excited state spectra were obtained by $\Delta \mathrm{A}=\log \left(\mathrm{I} / \mathrm{I}_{0}\right)$. Typically, 2000 excitation pulses were averaged to obtain the transient at a particular time. Due to the lenses, a chirp of $c a .1$ ps is observed between $460-$ $650 \mathrm{~nm}$. 


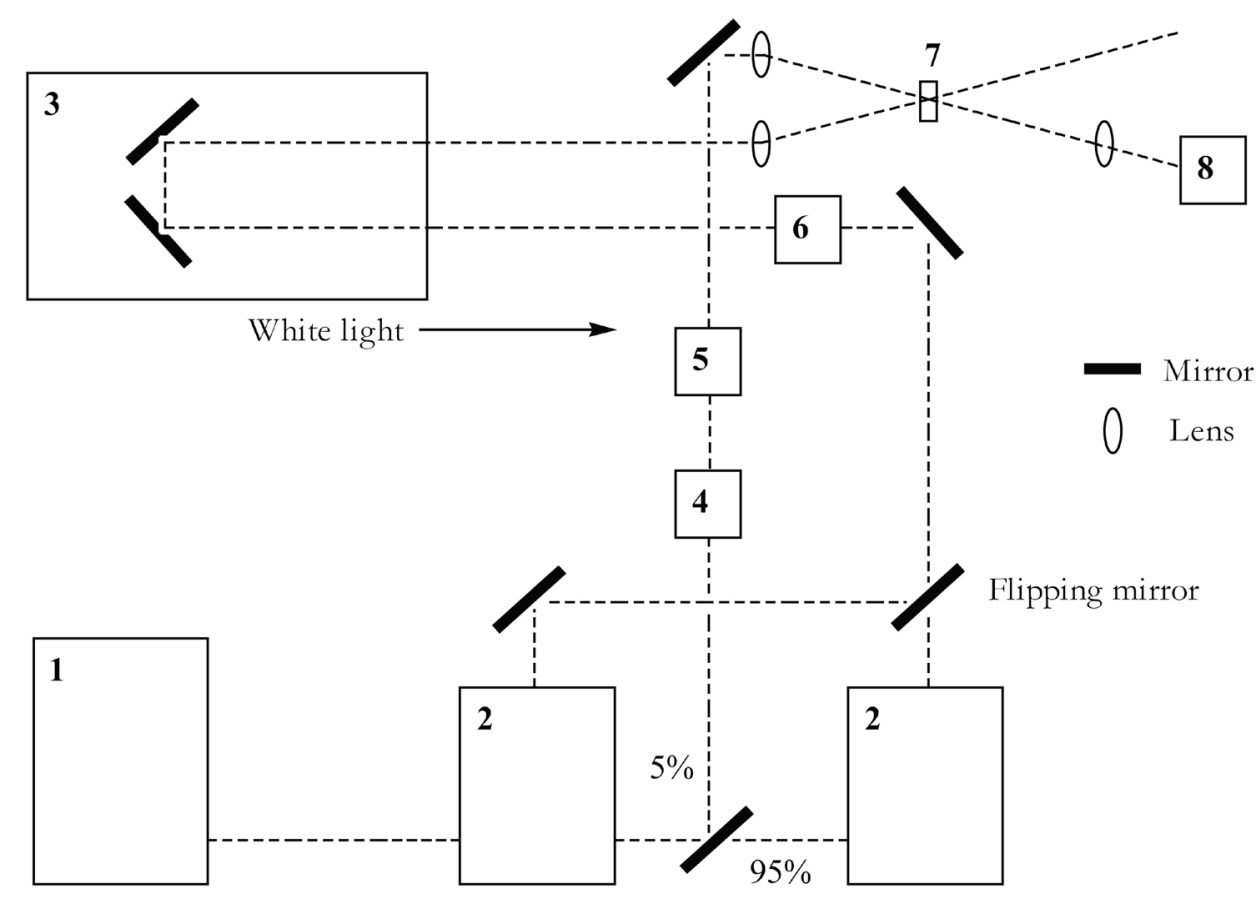

Figure 2.8. Schematic representation of the sub-picosecond transient absorption setup: 1. Hurricane, 2. OPA-800, 3. delay line, 4. white line generator, 5. Berek polarizer, 6. chopper, 7. sample and 8. CCD camera

\section{Confocal microscopy}

Confocal microscopy is one of the most powerful imaging techniques which allow us to probe various interactions at length scales of the order of the probing wavelength. In this thesis the confocal microscopy was mainly used for the fluorescence lifetime imaging (FLIM).In particular, FLIM was employed for probing energy transfer between NQDs and the dye molecules inside the zeolites. This technique was also employed to monitor the luminescence lifetimes of SAMs of $\mathrm{Ru}(\mathrm{II})$ on gold surfaces. 
The basic concept of a confocal microscope is shown in Figure 2.9. The excitation light from a coherent source (e.g. laser) passes through pinhole apertures before reaching the specimen. The laser light (dotted line in Figure 2.9 ) is reflected by a dichromatic mirror and scans a defined focal plane of the specimen. The secondary fluorescence (from a specimen in the same focal plane) is

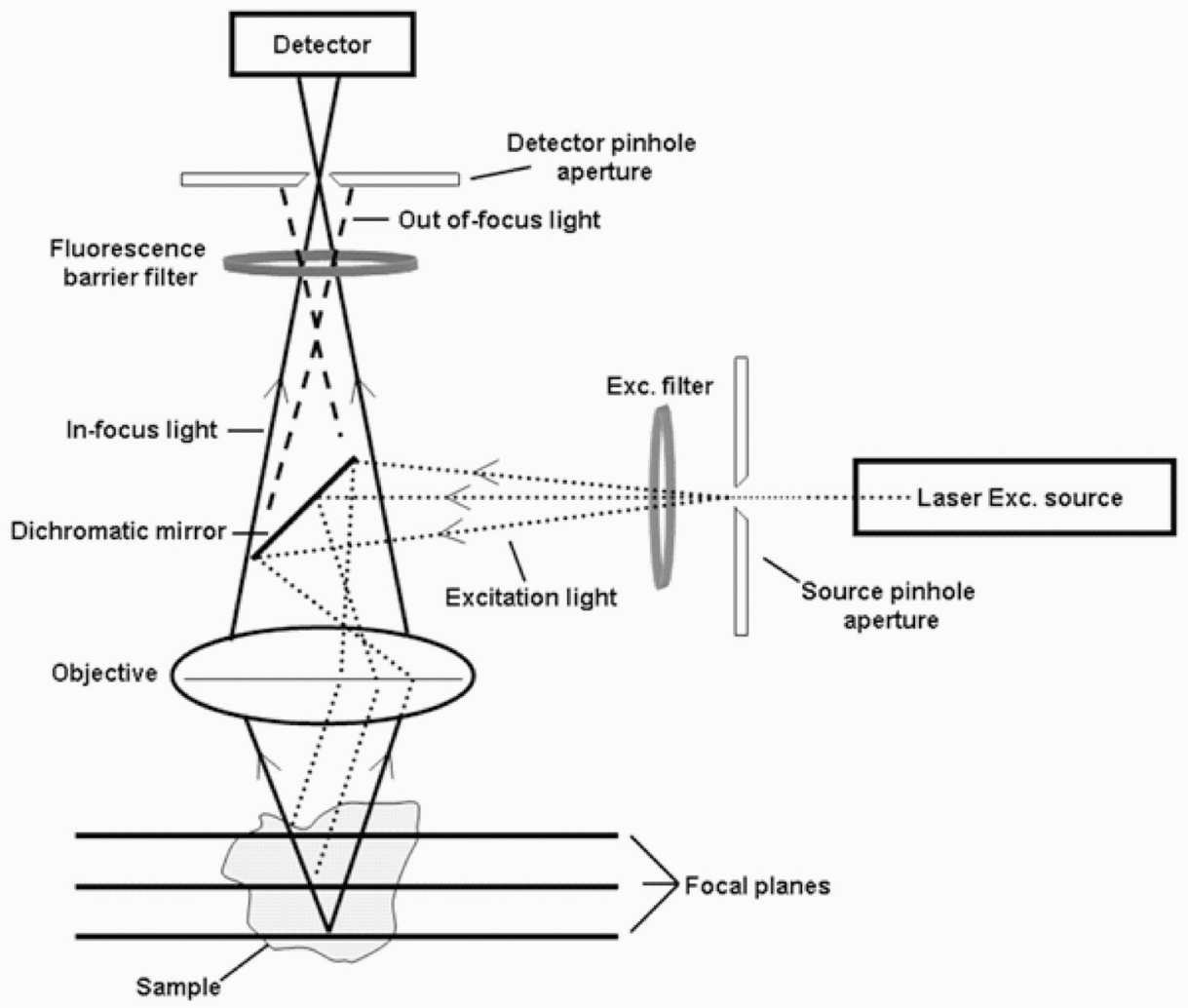

Figure 2.9. Schematic representation of a confocal microscope.

directed back through the objective, passes through the mirror and is focused on a confocal point of the detector pinhole aperture. Contrary to traditional epi-fluorescence wide field microscopes, where the whole sample is illuminated by a strong light source and can be directly observed through the eyepieces, the 
confocal microscope operates in a scanning mode. Modern confocal instruments are equipped with up to five laser systems, with scanning head, electronic detectors (photomultipliers) and a computer for acquisition and final image processing. Confocal microscopes cover the fluorescence emission range from 400 to $750 \mathrm{~nm}$. The FLIM images and the fluorescence decays on surfaces were recorded using a Microtime 200 (PicoQuant) attached to an Olympus IX 71 Microscope with a 100x Oil-immersion objective and a scanning speed of $6 \mu$ s per point at an excitation with a 440nm laser (FWHM $80 \mathrm{ps}$ ). Fluorescent lifetimes were calculated from the whole area by the software SymphoTime (PicoQuant).

\section{Atomic Force Microscopy}

Atomic force microscopy (AFM) is a very important tool in surface characterization. The information regarding the morphology of the surface and surface roughness could be obtained efficiently with this technique. In this thesis, for the surface characterization of the SAMs (chapter 4) was employed. AFM images of the monolayers on flat gold substrates were acquired in air at room temperature with a commercial instrument (Digital Instruments, Nanoscope IIIa, Dimension 3000, Santa Barbara, CA) operating in tapping mode. AFM images are flattened and shown without further modification. Analysis was performed using WSxM 4.0 Develop60.

\section{Cyclic Voltammetry}

Information regarding the first oxidation and reduction potentials of the molecules involved in the electron transfer is essential for the understanding of the photoinduced electron transfer process. In this thesis, cyclic voltammetry was used to measure the redox potentials of the metal complexes in solution (chapter 3, 4 and 5), and as SAMs (chapter 4). Cyclic Voltammetry (CV) was performed in a gastight single-compartment three-electrode cell using a Voltalab 40 system from Radiometer Analytical which consists of a PGZ301 potentiostat and Voltamaster 4 software. The working electrode was a Pt-disc, the counter electrode a Pt wire, and Ag wire was used as a pseudo-reference 
electrode. All glassware was dried prior to use. The compounds (electrolyte, analyte and reference) were placed in a Schlenk flask that was then evacuated and heated with a heat gun to eliminate any moisture and oxygen that had entered during the addition. The flask was then evacuated and filled three times with dry nitrogen. The solvent was added via syringe directly to the sealed Schlenk flask, and then degassed for ten minutes with a gentle stream of dry $\mathrm{N}_{2}$. After degassing, the solution was added, via syringe, to the electrochemical cell under a positive $\mathrm{N}_{2}$ pressure and the electrodes then added. The solution was kept under a positive $\mathrm{N}_{2}$ pressure during the measurements but no flow was allowed through the cell. For electrochemistry of surfaces, we used Ag and $\mathrm{Pt}$ wires as reference and counter-electrodes respectively. The subsequent experiments were carried out by using neat acetonitrile solution with tetrabutylammoniumhexafluorophosphate (Sigma Aldrich) as the electrolyte. Measurements with $\mathrm{Pt}$ functionalized with $\mathrm{Ru}-\mathrm{SAc}$ reported in chapter 4 were performed in the same experimental conditions as mentioned above.

\section{References}

1. Valeur, B., In Molecular Fluorescence: Principles and Applications, Wiley: Weinheim, 2001.

2. Demas, J. N.; Crosby, G. A., J. Phys. Chem. 1971, 75, 991.

3. Lackowicz, J. R., In Principles of Fluorescence Spectroscopy, 3rd ed.; Springer 2006; pp 281.

4. Bonneau, R.; Wirz, J.; Zuberbühler, A. D., Pure Appl. Chem. 1997, 69, 979.

5. Minsky, M., Scanning 1988, 10, 128. 


\title{
CHAPTER 3
}

SYNTHESIS AND PHOTOPHYSICAL PROPERTIES OF AMINO TERMINATED IRIDIUM AND RUTHENIUM COMPLEXES AND ENERGY TRANSFER WITH QUANTUM DOTS

\begin{abstract}
This chapter deals with the synthesis and photophysical characterization of polypyridyl complexes of $\operatorname{Ir}(\mathrm{III})$ and $\mathrm{Ru}(\mathrm{II})$ with one of the pyridines containing a terminal amino group which can act as anchoring unit for quantum dots. These complexes exhibit rich photophyics and electrochemistry that can be used to monitor photoinduced processes when the metal complexes are bound to an active surface. In particular the functionalization of $\mathbf{C d S e} / \mathrm{ZnS}$ quantum dots with Ru complex with amino functional group has been investigated. By employing a coordinated ligand exchange strategy, we effectively isolate the quantum dot ruthenium complex assembly and also demonstrate a photoinduced energy transfer from quantum dot to ruthenium complex. When the iridium complex was anchored onto the CdTe quantum dot surface a photoinduced energy transfer from metal complex to nanocrystals have been presented.
\end{abstract}




\section{Introduction}

Polypyridyl ruthenium and cyclometalated iridium complexes have been investigated since very long time. ${ }^{1-4}$ Their rich electrochemical and photophysical properties have attracted a lot of interest both from a fundamental as well as an application point of view. ${ }^{4-12}$ In particular the nature of the emissive states in these complexes has contributed significantly to the plethora of papers in the literature. Since these molecules are triplet emitters, they offer a potential advantage over conventional singlet organic emitters in various optoelectronic applications. To be specific, these complexes can harvest excitons from both singlet and triplet states giving them a theoretical efficiency of $100 \%$ charge recombination ${ }^{13}$. In contrast, from a statistical point of view, organic emitters can reach a maximum recombination efficiency of only $25 \%$. In addition to exhibiting long lived excited state, these complexes are electroluminescent which offers the prospect of using them in OLEDs ${ }^{14-18}$ and LEECs ${ }^{19-20}$ fabrication. One aspect common to these two classes of complexes is that in most cases the lowest electronic transition upon excitation involves a metal to ligand charge transfer state $\left({ }^{1}\right.$ MLCT) and that the emission takes place after intersystem crossing from a ${ }^{3}$ MLCT state. Both these classes of complexes exhibit a large Stokes' shift indicating that the ground and excited states have a different nature, and in particular different spin multiplicity. The HOMO LUMO gap can be tuned by varying the ligands that are coordinated to the metal centre. This effect is particularly strong for the iridium complexes which are much more sensitive to the coordination. Their stability, both chemical and photochemical is very remarkable and for some Iridium complexes emission quantum yields can reach more than $95 \%$. All these properties combined with a very versatile synthesis make the $\mathrm{Ru}(\mathrm{II})$ and $\mathrm{Ir}(\mathrm{III})$ class of complexes very appealing.

Quantum dots have been in the limelight for the past three decades because of their fascinating size dependent optical and electrical behavior owing to the quantum confinement of charge carriers ${ }^{21-24}$. This allows one to fabricate semiconducting materials with different band gaps by 
just varying their sizes which is not possible in bulk semiconductors ${ }^{25}$. In addition to the size tunability, these nanomaterials also exhibit a very narrow emission profile and are highly photostable which makes them a viable alternative to various fluorescent organic molecules for numerous applications like imaging in vitro $^{26-27}$, sensors ${ }^{28-29}$, lasers ${ }^{30-31}$ and optoelectronic devices ${ }^{32-39}$. These nanocrystals are synthesized by solvothermal methods yielding various sizes of quantum dots with high degree of monodispersity ${ }^{40}$. Conventionally these materials are synthesized with a coordinating surfactant like trioctylphosphine (TOP) or trioctylphosphineoxide (TOPO). In addition, they are also capped with ligands like dodecylamine (DDA), hexadecylamine (HDA) or oleic acid (OA). In absence of any capping ligands, the nanocrystals are unstable. The quantum dots have surface trap states that are highly sensitive to oxygen which is evident by the fact that upon exposure to oxygen, the surface of the quantum dots undergoes oxidation which would result in shrinkage in the size of the dots. This is mirrored in the change in the photophysical behavior wherein the emission spectrum undergoes an irreversible blue shift and the quantum efficiency falls significantly ${ }^{41-42}$. From an application point of view, the main drawbacks are their intrinsic toxicity and their stability only in inert atmosphere. To overcome these problems, the nanocrystals are coated with a few layers of wide band gap materials to effectively passivate the surface trap states $^{24,43-44}$. This would make these quantum dots stable against oxygen and also improve its quantum efficiency. However, in order to employ these nanoparticles for any optoelectronic application they have to be processable. Several reports deal with the exchange of the TOP and TOPO ligands with chromophores such as metal complexes of $\mathrm{Ru}^{45-50}$, $\mathrm{Ir}^{27}$ and $\mathrm{Os}^{51}$ bearing amino, thiol or carboxylic functionalities. This approach also allows the anchoring of photo- and/or electroactive units which can in combination with the QDs promote charge or energy transfer. These processes can be directionally controlled combining the appropriate QDs with the energy or electron acceptor or donor moieties. Such processes can lead to assemblies in which all the energy is funneled into a single component (light harvesting) or the charges are accumulated in a moiety and eventually used for chemical transformation. 


\section{Chapter 3}

In order to exploit such processes we have selected two candidates that can act as energy or charge acceptor or donor due to their chemical nature, electrochemical properties and photophysical behavior. This chapter deals with the synthesis of $\operatorname{Ir}(\mathrm{III})$ and $\mathrm{Ru}(\mathrm{II})$ complexes with amino functional groups as well as their photophysical characterization. Red emitting CdTe quantum dots coated with trioctylphosphine (TOP) and dodecyl amine (DDA) are used in combination with Ir(III) complex, and a photoinduced energy transfer from metal complex to the nanocrystals has been reported. The rationale behind using an electroluminescent molecule to sensitize quantum dots is to fabricate a nanoassembly wherein electrical excitation could be imparted indirectly to quantum dots. Such assemblies have potential applications in organic light emitting devices (OLEDs). ${ }^{52}$ By employing a coordinated ligand exchange strategy, core-shell quantum dots are functionalized with the $\mathrm{Ru}(\mathrm{II})$ complex and photoinduced processes in such nanoassemblies are investigated. To be specific, the ruthenium complex with a terminal amino group is coordinated to the blue emitting $\mathrm{CdSe} / \mathrm{ZnS}$ quantum dots surface and a photoinduced energy transfer is observed from the quantum dots to the metal complex. This assembly utilizes the high absorption cross section of quantum dots which act as light harvesting system able to funnel the energy to the lowest energy luminescent metal complex state. Such an assembly involving an antenna mechanism can serve as model system for light harvesting and activate a photoactive center in solar energy conversion. Energy transfer involving quantum dots has been studied in the past with an emphasis on biological applications ${ }^{53-55}$. In this chapter, two examples of energy transfer in which the QDs can act as donor or energy acceptor are reported.

The chapter will be divided in three sections, the first one dealing with the syntheses of the ligands and the Iridium complex, characterization and its assembly with quantum dots and the second one describing an analogous ruthenium complex with a different type of QD. In the last section a discussion about FRET in both these systems are presented. 


\section{Synthesis of $\left[\operatorname{Ir}(\mathrm{ppy})_{2}(\mathrm{bpy})-(\mathrm{ph})_{2}-\mathrm{NH}^{2}\right]^{+} \mathrm{PF}_{6}^{-}$}

Synthesis of neutral as well as charged iridium complexes has received a lot of attention over the years. The general procedure is shown in scheme 3.1. Synthesis of $\left[\operatorname{Ir}(\mathrm{ppy})_{2}(\mathrm{bpy})(\mathrm{ph})_{2} \mathrm{NH}_{2}\right]^{+} \mathrm{PF}_{6}^{-}\left(\mathbf{I r}-\mathbf{N H}_{2}\right)$ was carried out by following the procedure reported by Plummer et al. ${ }^{56}$ Starting from iridiumtrichloride hydrate, a dinuclear iridium complex is synthesized $^{57}$. From the dinuclear complex a cationic heteroleptic iridium compound with two phenyl spacers between the Ir core and the amine terminal group was synthesized.

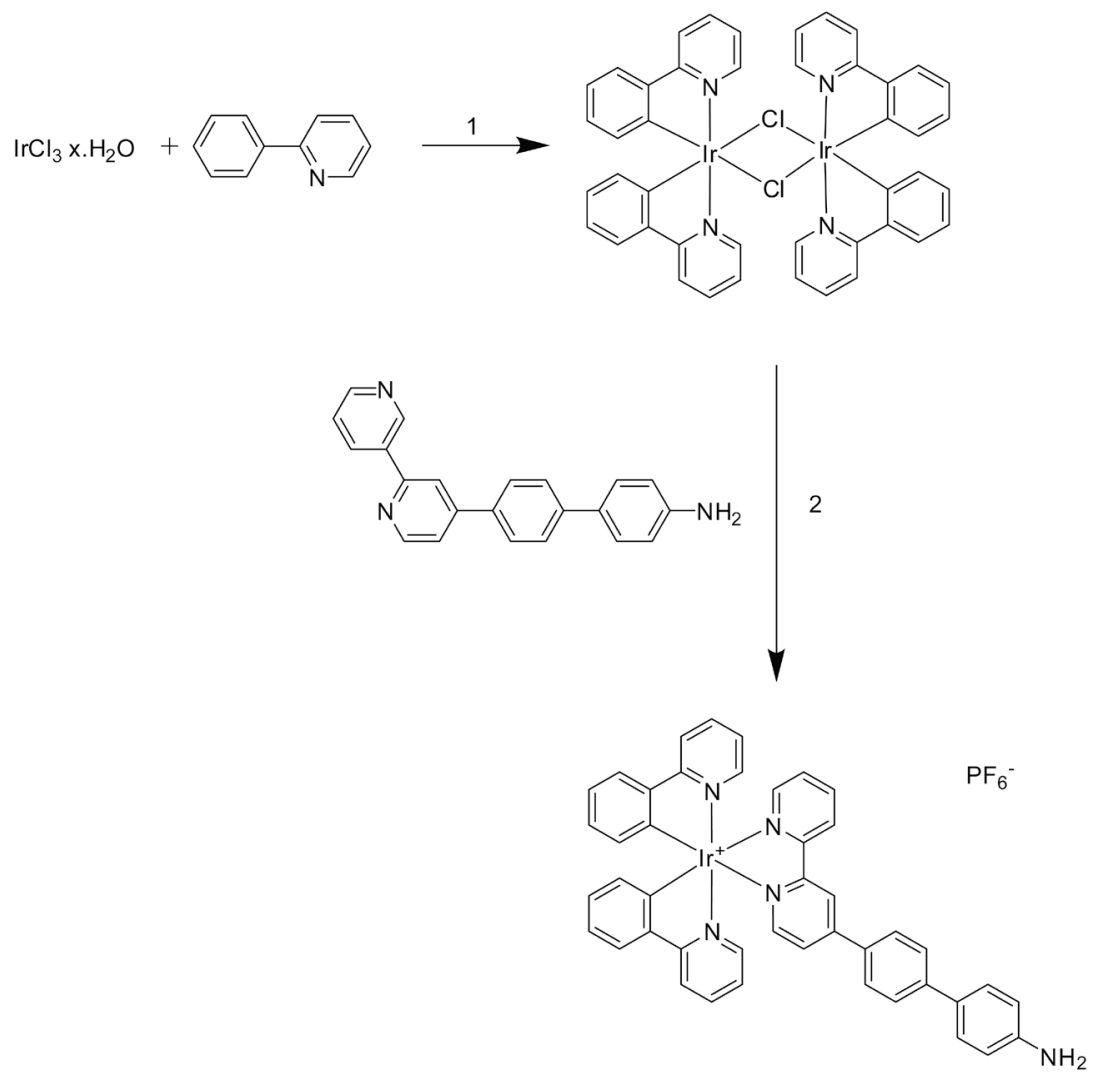

Scheme 3.1: Snthesis of $\left[\operatorname{Ir}(\mathrm{ppy})_{2}(\mathrm{bpy})(\mathrm{ph})_{2} \mathrm{NH}_{2}\right]^{+} \mathrm{PF}_{6}$. 1. Refluxed in 3:1 mixture of ethoxyethanol : water. 2.Refluxed in 1:3 mixture of methanol : dichloromethane under nitrogen atmosphere. 


\section{Photophysical properties of $\left[\operatorname{Ir}(\mathrm{ppy})_{2}(\mathrm{bpy})(\mathrm{ph})_{2} \mathrm{NH}_{2}\right]^{+}$ $\mathrm{PF}_{6}^{-}$}

The photophysical characterization of $\left[\mathrm{Ir}(\mathrm{ppy})_{2}(\mathrm{bpy})(\mathrm{ph})_{2} \mathrm{NH}_{2}\right]^{+}\left(\mathrm{PF}_{6}\right)^{-}$ $\left(\mathbf{I r}-\mathbf{N H}_{2}\right)$ is reported here in comparison with structurally similar $\left[\operatorname{Ir}(\mathrm{ppy})_{2}(\mathrm{bpy})\right]^{+} \quad\left(\mathrm{PF}_{6}\right)^{-} \quad$ (Ir-bpy). $\mathbf{I r}-\mathbf{N H}_{2}$ and $\mathbf{I r}-\mathbf{b p y}$ are both monocationic cyclometalated iridium complexes with $5 \mathrm{~d}^{6}$ electronic configuration. Figure 3.1 shows the steady state absorption spectra of Ir$\mathbf{N H}_{2}$ and Ir-bpy in acetonitrile solution. Both complexes exhibit similar absorption profiles in the UV region (around $255 \mathrm{~nm}$ ) with extinction coefficients of about $3.7 \times 10^{4} \mathrm{Lmol}^{-1} \mathrm{~cm}^{-1}$. This band system is attributed to the intraligand transitions (IL) $\left(\pi \rightarrow \pi^{*}\right)$ (phenylpyridyl and bipyridyl ligands $)^{58}$. The energetically lowest electronic transitions in Ir-bpy (around $380 \mathrm{~nm}$ ) involve much weaker metal to ligand charge transfer $\left({ }^{1}\right.$ MLCT) with an absorption coefficient of $10^{3} \mathrm{Lmol}^{-1} \mathrm{~cm}^{-1}\left(\mathrm{~d} \pi \rightarrow \pi^{*}\right)$. The ${ }^{1}$ MLCT bands are related to the transitions from the $\mathrm{d}$ orbital of the Ir and the $\pi^{*}$ states of the bypyridyl ligand. The lowest absorptions are due to the singlet to triplet MLCT bands which for Ir as well as for other heavy atoms are partially allowed, because of the strong spin-orbit coupling $^{58-60}$ and can be observed between 380 and $450 \mathrm{~nm}$. However, in case of $\mathbf{I r}-\mathbf{N H}_{2}$ a stronger band $\left(\varepsilon=14500 \mathrm{Lmol}^{-1} \mathrm{~cm}^{-1}\right)$ around $380 \mathrm{~nm}$ is seen as compared to the reference compound. The origin of this absorption feature is attributed to the transitions involving the two phenyl rings. In fact this stronger band masks the much less intense ${ }^{1}$ MLCT band. 


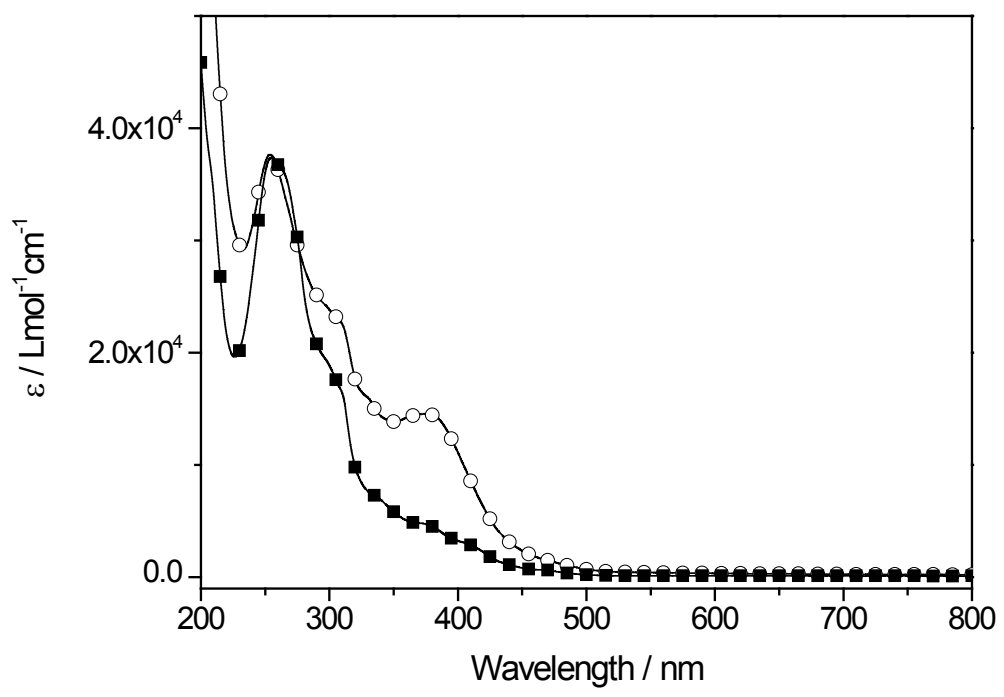

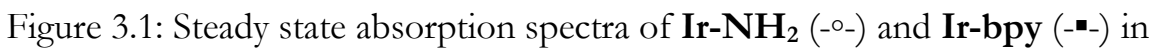
acetonitrile solution

Upon irradiation, in acetonitrile solution both complexes exhibit orange luminescence with $\mathbf{I r}-\mathbf{N H}_{2}$ at $585 \mathrm{~nm}$ and $\mathbf{I r}-\mathbf{b p y}$ at $602 \mathrm{~nm}$. Once excited, the complex undergoes an intersystem crossing from ${ }^{1} \mathrm{MLCT}$ to ${ }^{3}$ MLCT and emission originates from the spin forbidden transition from this energy level to the singlet ground state. The emission profile is very broad as shown in Figure 3.2 which is typical for the ${ }^{3}$ MLCT emission due to a significant shift of the excited state nuclear coordinated relative to the ground state. The low temperature spectrum for $\mathbf{I r}-\mathbf{N H}_{2}$ measured at $77 \mathrm{~K}$ in a butyronitrile matrix (inset figure 3.2) shows a maximum at $553 \mathrm{~nm}$. This blue shift at low temperature is typical for complexes involving MLCT transitions having the excited state more polar than the ground state. The solvent, frozen at this temperature cannot stabilize the polar excited state and therefore a destabilization of the excited state occurs and hence inducing a blue shift of the emission. At $77 \mathrm{~K}$, even the excited state lifetimes of the two compounds vary drastically; $\mathbf{I r}-\mathbf{N H}_{2}$ has a phosphorescence lifetime of $83 \mu$ s as compared to the reference compound, Ir-bpy which has an excited state lifetime of 
$5.4 \mu \mathrm{s}$. This effect is also reflected in the difference in the radiative and non radiative rate constants of $\mathbf{I r}-\mathbf{N H}_{2}$ and $\mathbf{I r - b p y}$ (Table 3.1). This difference could be explained by considering the lowest excited states. In solution $(298 \mathrm{~K})$, the emission is mainly from ${ }^{3} \mathrm{MLCT}$ state which is the lowest excited state. However, at $77 \mathrm{~K}$ since these states are strongly destabilized due to the absence of any solvent reorganization, the emission occurs from a ${ }^{3} \mathrm{LC}$ state which would be the lowest excited state and these ligand centered states typically live much longer. The complete photophysical data are listed in table 3.1. Compared to the reference compound, Irbpy, the emission of $\mathbf{I r}-\mathbf{N H}_{2}$ is about $17 \mathrm{~nm}$ blue shifted. This can be explained by the electron donating effect exerted by the amino group towards the bpy fragment which increases the energy of the emissive state. This leads to a destabilization of the LUMO of $\mathbf{I r}-\mathbf{N H}_{2}$ which in turn increases the HOMO - LUMO gap thus results in a blue shift. Even though there are two phenyl rings which should induce a red shift of the emission due to extended conjugation, the effect exerted by the amino group is predominant. This is evident from the fact that the emission maximum of $\mathbf{I r}-\mathbf{N H}_{2}$ lies in between that of $\mathbf{I r}$ (bpy) $(602 \mathrm{~nm})$ and $\mathbf{I r}(\text { ppy })_{2}$ (bpy)- $\mathbf{N H}_{2}(553 \mathrm{~nm}) .{ }^{61}$ 


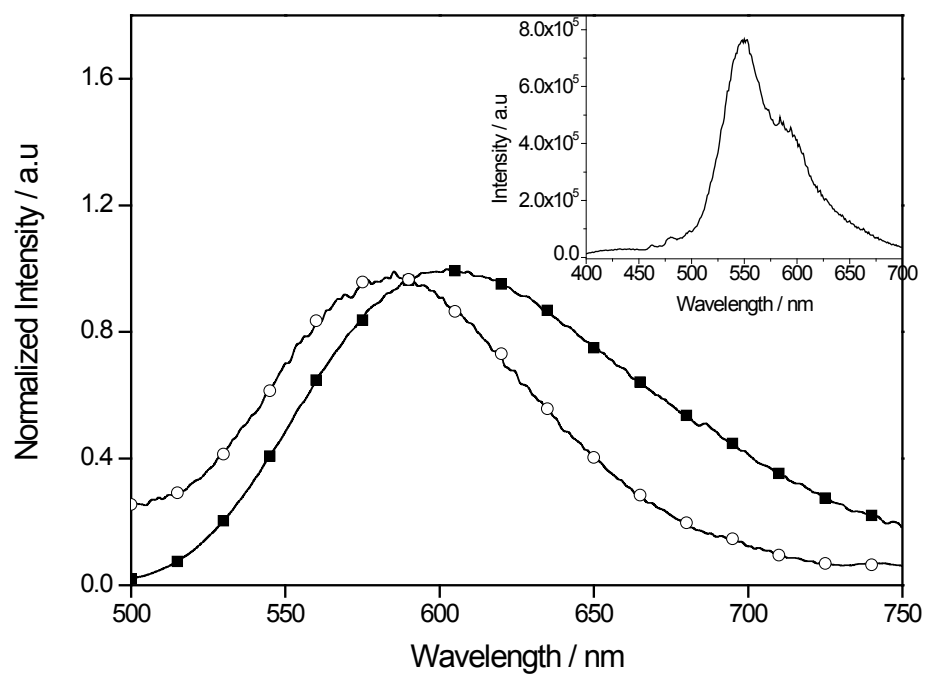

Figure 3.2: Steady state emission spectra of $\mathbf{I r}-\mathbf{N H}_{2}\left(-_{-}^{-}\right)$and $\mathbf{I r}-\mathbf{b p y}\left(\mathbf{- -}_{-}\right)$in acetonitrile solution. $\left(\lambda_{\mathrm{ex}}=375 \mathrm{~nm}\right)$. Inset: Emission spectra of $\mathbf{I r}-\mathbf{N H}_{2}$ measures at $77 \mathrm{~K}$ in butyronitrile matrix.

The emission quantum yield of this complex is also much lower than that of the reference compound. This result can be explained by the presence of the two phenyl rings. It is reported that upon excitation, the biphenyl moiety tends to rotate freely with a torsional angle that ranges between 70 to $160^{\circ 62}$. This free rotation introduces additional pathways for non-radiative deactivation to the ground state, thereby decreasing the quantum yields of the complexes containing biphenyl units. 


\section{Chapter 3}

\begin{tabular}{|c|c|c|c|c|c|c|c|c|c|}
\hline & \multicolumn{7}{|c|}{ Emission, $298 \mathrm{~K}^{a}$} & \multicolumn{2}{|c|}{$\begin{array}{c}\text { Emission, } 77 \\
\mathrm{~K}^{d}\end{array}$} \\
\hline Complex & $\begin{array}{c}\lambda_{\max } \\
(\mathrm{nm})^{b}\end{array}$ & $\begin{array}{c}\Phi \\
\text { deaer }^{c}\end{array}$ & $\begin{array}{c}\Phi \\
\text { aerated }^{c}\end{array}$ & $\begin{array}{l}\tau(\mu \mathrm{s}) \\
\text { deaer }\end{array}$ & $\begin{array}{c}\tau(\mu \mathrm{s}) \\
\text { aerated }\end{array}$ & $\begin{array}{c}k_{r} \\
\left(10^{5}\right. \\
\left.\mathrm{s}^{-1}\right)\end{array}$ & $\begin{array}{c}k_{n r} \\
\left(10^{5}\right. \\
\left.\mathrm{s}^{-1}\right)\end{array}$ & $\begin{array}{c}\lambda_{\max } \\
(\mathrm{nm})^{b}\end{array}$ & $\tau(\mu \mathrm{s})$ \\
\hline $\mathrm{Ir}-\mathrm{NH}_{2}$ & 585 & 0.02 & 0.001 & 1.6 & 0.326 & 0.125 & 61.25 & 553 & 83.8 \\
\hline Ir-bpy & 602 & 0.075 & 0.008 & 1.2 & 0.355 & 0.625 & 7.6 & 532 & 5.24 \\
\hline
\end{tabular}

Table 3.1: Luminescence data of $\mathbf{I r}-\mathbf{N H}_{2}$ and Irbpy at RT as well as at $77 \mathrm{~K}$.

\section{Electrochemistry}

Cyclometalated $\operatorname{Ir}(\mathrm{III})$ complexes are known to exhibit good redox behavior. The electrochemistry of Ir-bpy is thoroughly documented in the literature. The reference iridium complex exhibits an oxidation peak at $0.88 \mathrm{~V}$ and a reduction peak at $-1.81 \mathrm{~V}$ vs. ferrocene/ferrocene ${ }^{2+}$. $^{63}$ Both of these peaks are reversible. The oxidation is attributed to the metal center whereas the one electron reduction potential is attributed to the reduction of the bpy ligand. On the contrary, $\mathbf{I r}-\mathbf{N H}_{2}$ shows poor electrochemical behavior both in oxidation as well as in reduction. This complex shows an irreversible oxidation peak at $0.89 \mathrm{~V}$ vs. ferrocene/ferrocene $e^{2+}$. This peak is attributed to the oxidation of the metal center and the irreversibility could be possibly due to the presence of the electron donating amino group although this fact is not clearly understood yet. In reduction, it exhibits two peaks at $-1.24 \mathrm{~V}$ and -1.855 $\mathrm{V}$ respectively. The former peak is irreversible whereas the latter is quasi reversible as can be seen from Figure 3.3. The latter could be attributed to the reduction of the bpy ligand coordinated to the metal center as this value is similar to the one reported for the reference Ir-bpy compound. 


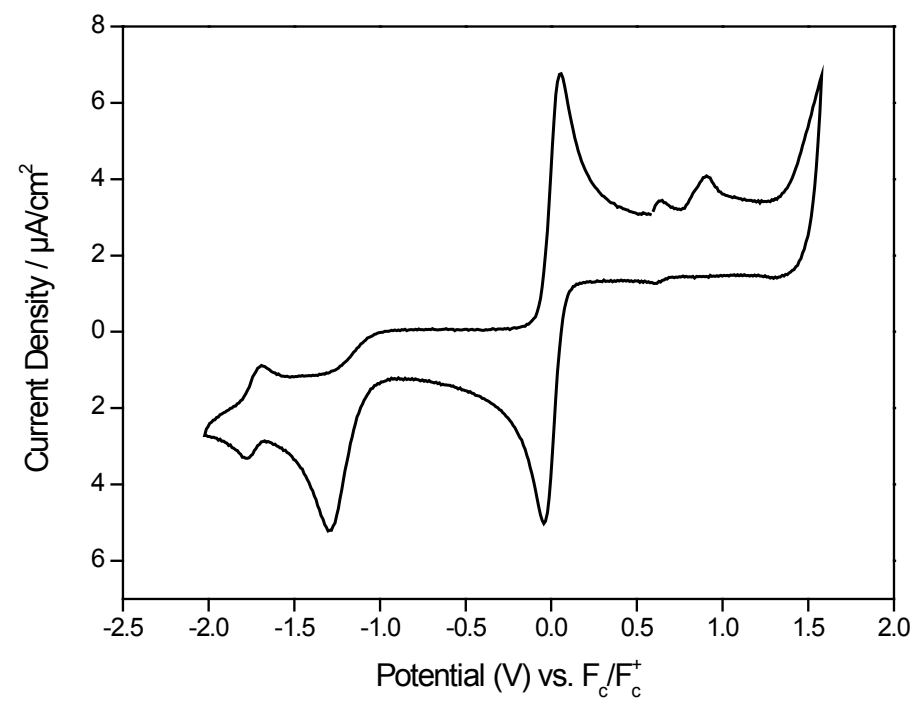

3.3: Cyclic voltammogram of $\mathbf{R u}-\mathbf{N H}_{2}$ in neat acetonitrile solution measured against ferrocene/ferrocene ${ }^{+}$redox couple.

\section{CdTe quantum dots}

For the studies involving red emitting quantum dots and $\operatorname{Ir}(\mathrm{III})$ complex, the nanocrystals were synthesized in Prof. Daniel Vanmaekelbergh's group at the University of Utrecht. The detailed synthesis and characterization of these QDs are reported elsewhere. ${ }^{64}$ These nanocrystals are capped with TOP and DDA. The concentration of the quantum dot dispersion in dichloromethane was estimated from absorption measurements following literature procedures. ${ }^{65} \mathrm{~A}$ brief description of the determination of the concentration of CdTe is presented in the experimental section. Figure 3.4 represents the absorption and emission spectra of CdTe quantum dots dispersed in DCM. These nanocrystals have very broad absorption but a narrow emission and relatively smaller Stoke shift due to the direct recombination of the charge carriers from conduction band to valence band. ${ }^{21}$ The band edge absorption is at $588 \mathrm{~nm}$ with a molar absorptivity 
coefficient of $3.17 \times 10^{5} \mathrm{~L} \cdot \mathrm{mol}^{-1} \cdot \mathrm{cm}^{-1}$, and for these nanocrystals the exciton emission is at $620 \mathrm{~nm}$. The weak emission above $675 \mathrm{~nm}$ as canbe seen from Figure 3.4 is due to the defect emission of these nanocrystals. The emission quantum yield of these nanocrystals is determined to be about 0.3. These quantum dots exhibit multiexponential decay due to the size distribution and surface trap states. ${ }^{66}$ The average lifetime of these quantum dots in DCM was measured to be 9.0 ns.

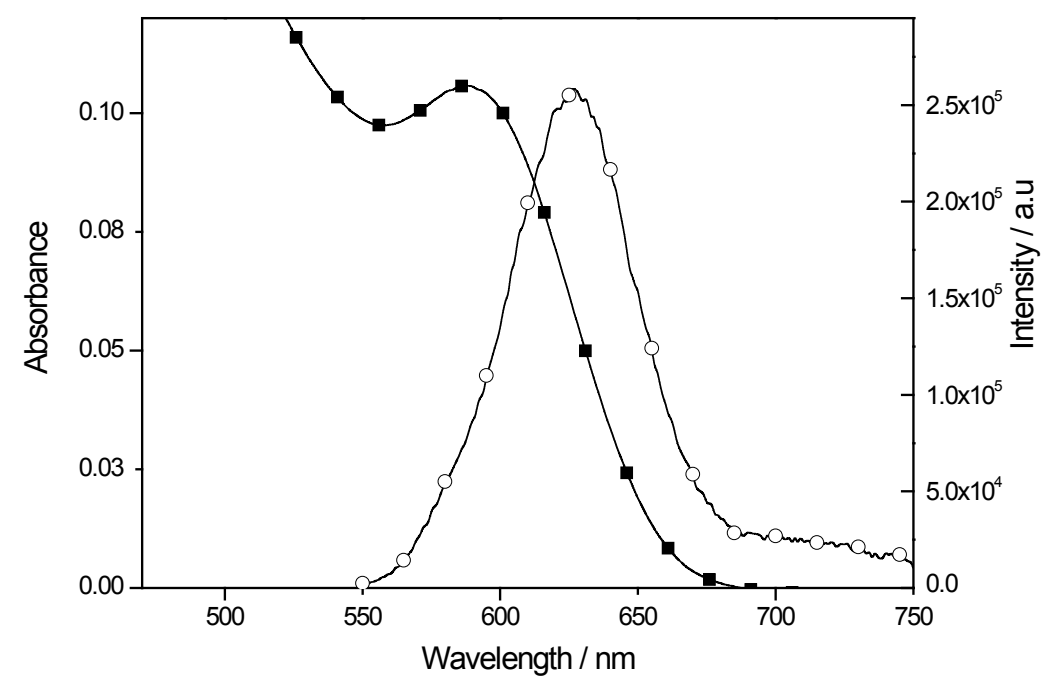

3.4: Absorption and emission spectra of CdTe quantum dots in DCM. $\lambda_{\text {ex }}=$ $402 \mathrm{~nm}$.

\section{$\mathrm{CdTe} / \mathrm{Ir}-\mathrm{NH}_{2}$ nanoassemblies}

CdTe nanocrystals have an affinity to bind to ligands like thiols, amines, phosphonates and carboxylates. The present batch of quantum dots which were synthesized with TOP and DDA. These nanocrystals were washed with methanol to create some vacancies on the surface by eliminating some of the initially capped TOP and DDA. About 10 fold 
excess of $\mathbf{I r}-\mathbf{N H}_{2}$ was added to the quantum dot suspension and the mixture was stirred for one hour so that the metal complex could coordinate to the nanocrystal surface through amine site by displacing some capping ligands. The solutions/suspensions were prepared in such a way that the concentrations of the metal complex and quantum dots in the assembly were identical to the reference solutions. The choice of the specific quantum dots used for the present study is dictated by the excellent spectral overlap with the metal complex (Figure 3.5). Due to the instability of the quantum dots in oxygen atmosphere, all the experiments were carried out in a glove box.

\section{Energy transfer studies in CdTe/ $\mathbf{I r}-\mathrm{NH}_{2}$ nanoassemblies}

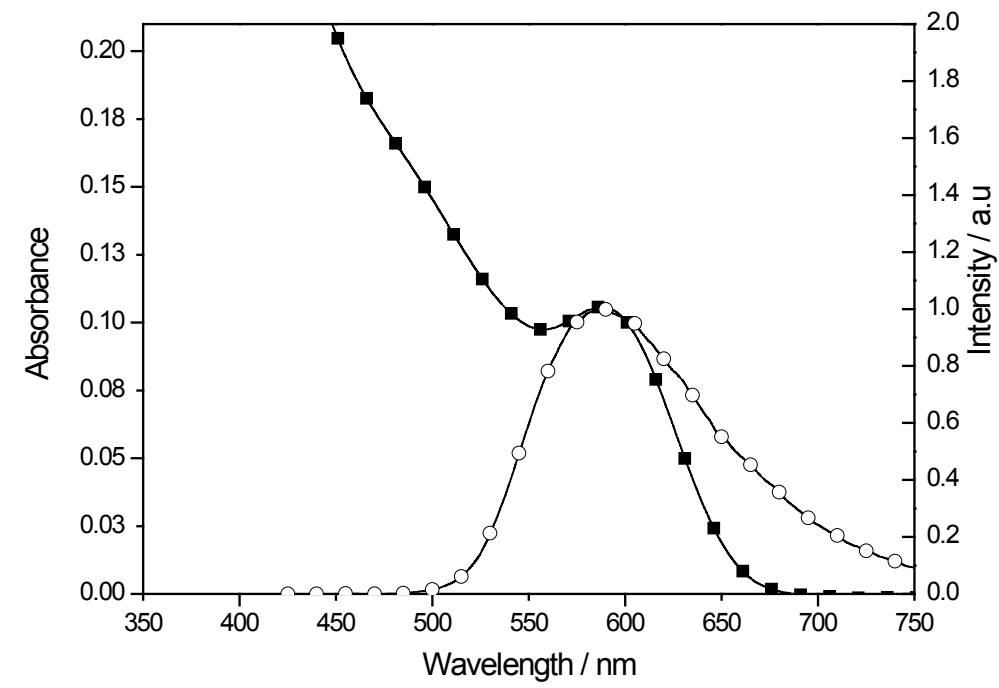

3.5: Spectral overlap between Ir- $\mathbf{N H}_{2}$ (donor -- - ) and $\mathbf{C d T e}$ (acceptor -o-)

For the energy transfer studies, the concentration of CdTe was maintained at $0.5 \mu \mathrm{M}$ and $\mathbf{I r}-\mathbf{N H}_{2}$ at $5 \mu \mathrm{M}$. Figure 3.6 presents the absorption spectra of $\mathbf{C d T e}, \mathbf{I r}-\mathbf{N H}_{2}$ and the assembly recorded in dichloromethane. The absorption of the assembly is a numerical sum of 
both individual components indicating that the two species possibly do not interact in ground state

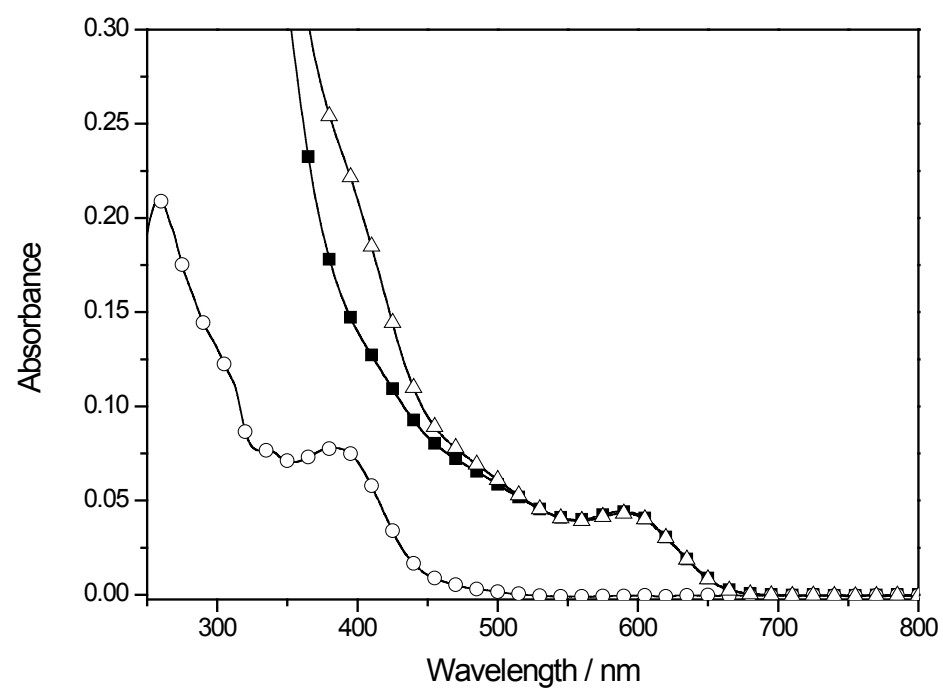

Figure 3.6: Absorption spectra of $\mathbf{C d T e}(---), \mathbf{I r}-\mathbf{N H}_{2}\left({ }^{-}{ }_{-}\right)$and $\mathbf{C d T e} / \mathbf{I r}-\mathbf{N H}_{2}$ assembly $(-\Delta-)$ in dichloromethane

The emission spectra of $\mathbf{C d T e}, \mathbf{I r}-\mathbf{N H}_{2}$ and the assembly are shown in Figure 3.7. The spectra were excited at $402 \mathrm{~nm}$. Since quantum dots absorb all the wavelengths below their exciton absorption, it is impossible to selectively excite the donor when the nanocrystals are energy acceptors. However, the concentration of CdTe and $\mathrm{Ir}-\mathrm{NH}_{2}$ were identical to their respective reference solutions so that the amount of light absorbed by each component is same in the assembly and reference solutions. 

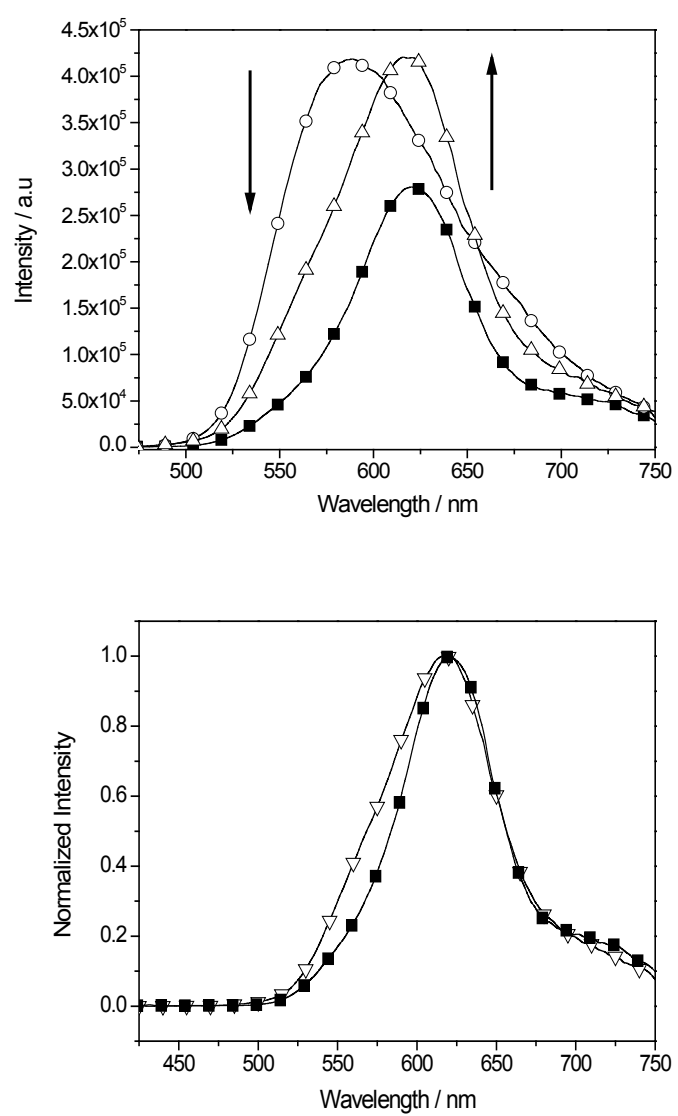

Figure 3.7: (Top) Emission spectra of CdTe (--), Ir- $\mathbf{N H}_{2}$ (-०-) and CdTe/Ir$\mathbf{N H}_{2}$ assembly $\left(-\Delta^{-}\right)$in dichloromethane. $\lambda_{\mathrm{em}}=402 \mathrm{~nm}$. (Bottom) normalized emission spectra of $\mathbf{C d T e}$ and the assembly.

The spectrum of the assembly has contributions from both $\mathbf{I r}-\mathbf{N H}_{2}$ and CdTe and it is difficult to deconvolute them due to the spectral overlap. However, it is evident from the spectra that the $\operatorname{Ir}(\mathrm{III})$ emission is quenched significantly and the quantum dots are sensitized. Since the spectral profile of CdTe and the assembly look the same it is important to consider that the broadening of the assembly spectrum is not due to the increase in intensity. This possibility can be ruled out by considering the normalized emission spectra of both CdTe and the assembly as shown in the right panel of Figure 3.6. It is clearly distinguishable that 
the broadening of the spectrum is indeed due to the contribution from the metal complex. The extent of quenching of iridium complex was determined by measuring the excited state lifetimes of individual components and the assembly using a time correlated single photon counting (TCSPC) system and the results are tabulated in Table 3.2. It is evident that the lifetime of the assembly is quenched from $1.4 \mu$ s to 849 ns. The energy transfer efficiency could be calculated using the equation

$$
E=1-\frac{\tau}{\tau_{0}}
$$

where $\tau$ and $\tau_{0}$ are the excited state lifetimes of the donor in absence and in presence of the acceptor moiety. For the CdTe-Ir- $\mathrm{NH}_{2}$ system, E was calculated to be about $40 \%$.

Table: 3.2. Excited-state Lifetimes ${ }^{a}$ of CdTe-Ir(III) assemblies

\begin{tabular}{|c|c|c|c|c|c|c|}
\hline Compound & $\tau_{1}$ & Rel. amp. & $\tau_{2}$ & Rel. & $\tau_{3}$ & Rel. \\
\hline CdTe & $5.4^{\mathrm{b}}$ & 42 & 14.9 & 43 & 1.5 & 15 \\
\hline Ir-NH ${ }_{2}$ & $1408^{c}$ & 100 & - & - & - & - \\
\hline \multirow[t]{2}{*}{$\begin{array}{c}\text { CdTe-Ir- } \\
\mathrm{NH}_{2} \\
\end{array}$} & $849 c$ & 100 & - & - & - & - \\
\hline & $5.9^{\mathrm{b}}$ & 44 & 15 & 45 & 1.9 & 11 \\
\hline Ir-bp & $1180^{c}$ & 100 & - & - & - & - \\
\hline \multirow[t]{2}{*}{ CdTe-Ir-bp } & $1100^{c}$ & 100 & - & - & - & - \\
\hline & 6 & 47 & 15.9 & 34 & 1.9 & 19 \\
\hline
\end{tabular}

In order to rule out any long range interactions between the quantum dots and the metal complexes or any trivial energy transfer in these systems, a control experiment was carried out with Ir-bp which has the same Ir(III) core but lacks any anchoring groups. However, in this experiment we did not observe any sensitization (Figure 3.8) of CdTe although the spectral overlap is very much similar. The excited state lifetimes are tabulated in table 3.2 and no reduction of $\operatorname{Ir}(\mathrm{III})$ lifetime 
was observed as well. This control experiment proves that what we observe in case of $\mathbf{C d T e} / \mathbf{I r}-\mathbf{N H}_{2}$ is a photoinduced energy transfer. Detailed energy transfer discussion is presented later in the chapter.

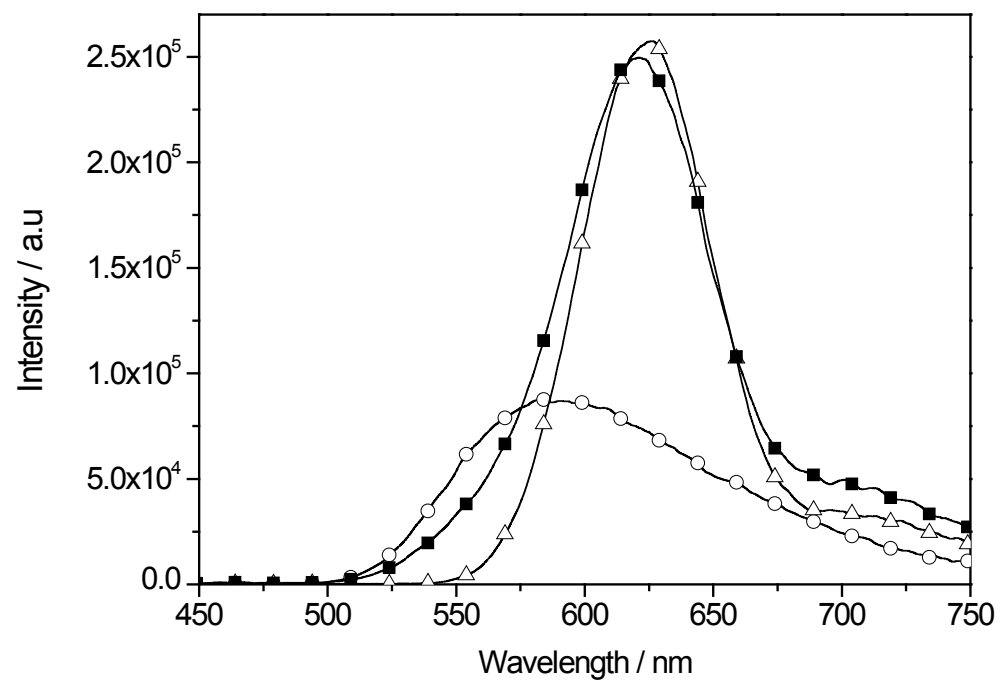

Figure 3.8: Emission spectra of CdTe (--), Ir-bpy (-o-) and CdTe/Ir-bpy mixture $(-\Delta-)$ in dichloromethane. $\lambda_{\mathrm{em}}=402 \mathrm{~nm}$.

\section{Synthesis of $\left[\mathrm{Ru}(\mathrm{bpy})_{2}(\mathrm{bpy})-(\mathrm{ph})_{2}-\mathrm{NH}_{2}\right]^{2+} 2\left(\mathrm{PF}_{6}\right)^{-}$}

Over the past years several routes have been reported in the literature for synthesizing ruthenium polypyridyl complexes ${ }^{67-73}$. The synthesis route followed for the above mentioned iridium complex (scheme 3.1) was employed to begin with starting from $\mathrm{RuCl}_{3}$ and 2,2' bipyridine ligands. This resulted in obtaining the final product with very poor yield. So, a three step synthesis (Scheme 3.2) as reported by Welter et al. ${ }^{74}$ starting with $\left[\mathrm{Ru}(\mathrm{bpy})_{2} \mathrm{Cl}_{2}\right]$ and complexing this with the 4,(4'-bromophenyl)$\left[2,2^{2}\right]$ bipyridynyl ligand in ethylene glycol under microwave irradiation $(450 \mathrm{~W})$ was performed. By employing a $\mathrm{Pd}(0)$ catalyzed Suzuki cross coupling reaction, the final product was obtained in good yields ${ }^{73}$ (about $85 \%)$. 


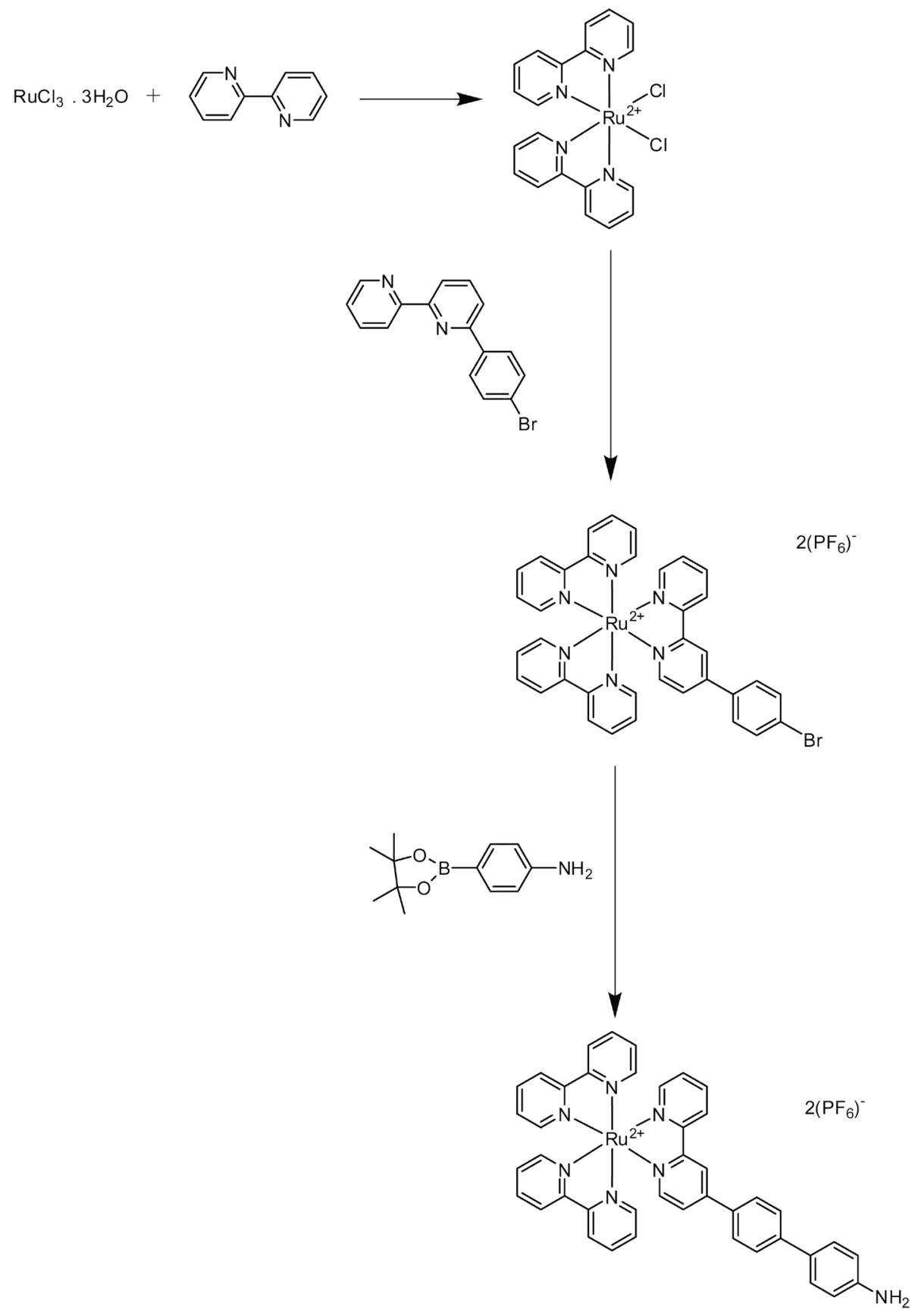

Scheme 3.2: Synthesis of Synthesis of $\left[\mathrm{Ru}(\mathrm{bpy})_{2}(\mathrm{bpy})-(\mathrm{ph})_{2}-\mathrm{NH}_{2}\right]^{2+} 2\left(\mathrm{PF}_{6}\right)^{-} \cdot 1$ : $\mathrm{LiCl}$, reluxed in DMF for 8 hours. 2: ethyleneglcol, microwave $(450 \mathrm{~W})$ for 5 minutes. 3: $\mathrm{Pd}\left(\mathrm{PPh}_{3}\right)_{4}, \mathrm{CsCO}_{3}$ heated at $94^{\circ} \mathrm{C}$ for 20 hours. 


\section{$\left[\mathrm{Ru}(\mathrm{bpy})_{2}(\mathrm{bpy})-(\mathrm{ph})_{2}-\mathrm{NH}_{2}\right]^{2+2}\left(\mathrm{PF}_{6}\right)^{-}$}

The photophysical characterization of $\left[\mathrm{Ru}(\mathrm{bpy})_{2}(\mathrm{bpy})-(\mathrm{ph})_{2}-\mathrm{NH}_{2}\right]^{2+} 2\left(\mathrm{PF}_{6}\right)-$ $\left(\mathbf{R u}-\mathbf{N H}_{2}\right)$ presented in this chapter is compared to that of $\mathbf{R u}(\mathbf{b p y})_{3}$ since the two complexes have a high structural similarity. $\mathbf{R u}(\mathbf{b p y})_{3}$ is probably the most well studied organometallic complex from a photophysical point of view. The steady state absorption spectra of Ru$\mathbf{N H}_{2}$ and $\mathbf{R u}(\text { bpy })_{3}$ are presented in Figure 3.9. The intense absorption bands around $290 \mathrm{~nm}$ for both complexes are attributed to intra ligand electronic transitions involving the bpy ligands (IL) $\left(\pi \rightarrow \pi^{*}\right)$. Both complexes possess a low energy transition around $450 \mathrm{~nm}$ that arises due to the metal to ligand charge transfer $\left(\mathrm{d} \pi \rightarrow \pi^{*}\right)\left({ }^{1} \mathrm{MLCT}\right)$ transitions. The less intense bands around $240 \mathrm{~nm}$ for both the complexes are attributed to higher MLCT transitions. In case of $\mathbf{R u}(\mathbf{b p y})_{3}$, the very weak transition around $344 \mathrm{~nm}$ is attributed to the metal centered (MC) transition. However, in case of $\mathbf{R u}-\mathbf{N H}_{2}$, this band is masked by a more pronounced shoulder which originates from the transitions involving the two phenyl moieties.

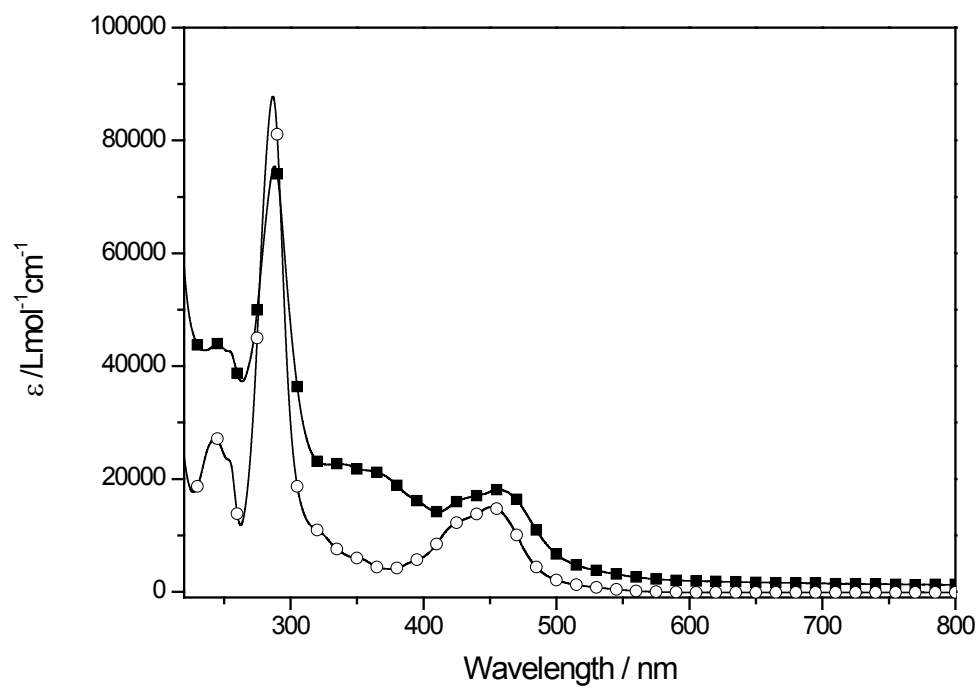

Figure 3.9: Steady state absorption spectra of $\mathrm{Ru}^{-\mathrm{NH}_{2}}\left(--_{-}\right)$and $\mathrm{Ru}(\mathrm{bpy})_{3}\left(-_{-}\right)$in acetonitrile solution. 
Exciting these complexes in a solution of acetonitrile at their absorption maxima (MLCT) would result in a broad emission profile which is characteristic of complexes having MLCT emissions. The absorption maximum for $\mathbf{R u}-\mathbf{N H}_{2}$ is at $620 \mathrm{~nm}$ and for $\mathbf{R u}(\text { bpy) })_{3}$ is at $621 \mathrm{~nm}$ (Figure 3.9). Luminescent properties of $\mathbf{R u}-\mathbf{N H}_{2}$ and $\mathbf{R u}(\mathbf{b p y})_{3}$ are listed in Table 3.3. The emissions of both the complexes occur from ${ }^{3}$ MLCT level. Typical for such complexes, the emission is highly quenched by molecular oxygen as is evident from the excited state lifetimes and quantum yields measured in deaerated acetonitrile solutions. In comparison with $\mathbf{R u}(\mathbf{b p y})_{3}, \mathbf{R u}-\mathbf{N H}_{2}$ has relatively low quantum yield. This could again be explained on the basis of presence of a biphenyl unit which could rotate freely as explained earlier for the Ir complex. At $77 \mathrm{~K}, \mathbf{R u}-\mathbf{N H}_{2}$ has a structured emission profile and a very long lifetime of $6.4 \mu$ s with the emission blue shifted by about $30 \mathrm{~nm}$ with respect to the RT emission. These findings can be explained as follows. For ${ }^{3}$ MLCT emissions which are solvatochromic due to the charge transfer character, the excited state is always stabilized by the solvent molecules. In the solid matrix, since the solvent is frozen, the solvent reorganization is absent and hence this stabilization is not possible. Therefore the HOMO - LUMO gap increases resulting in the blue shift of the emission as observed for these complexes ${ }^{3,75}$. 


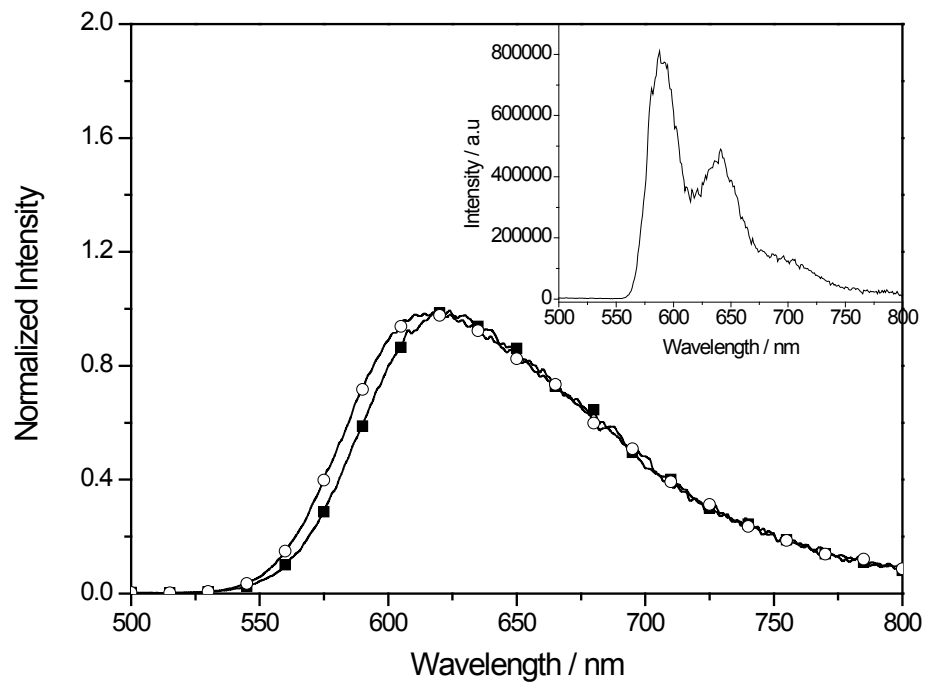

3.10: Steady state emission spectra of $\mathbf{R r}-\mathbf{N H}_{2}\left({ }_{-}-_{-}\right)$and $\mathbf{R u}(\mathbf{b p y})_{3}$ (--) in acetonitrile solution. ( $\lambda_{\mathrm{ex}}=450 \mathrm{~nm}$ ). Inset: Emission spectra of $\mathbf{R u}-\mathbf{N H}_{\mathbf{2}}$ measured at $77 \mathrm{~K}$ in butyronitrile matrix.

\begin{tabular}{|c|c|c|c|c|c|c|c|c|c|}
\hline & \multicolumn{7}{|c|}{ Emission, $298 \mathrm{~K}^{a}$} & \multicolumn{2}{|c|}{$\begin{array}{c}\text { Emission, } \\
77 \mathrm{~K}^{d}\end{array}$} \\
\hline Complex & $\begin{array}{c}\lambda_{\max } \\
(\mathrm{nm})^{b}\end{array}$ & $\begin{array}{c}\Phi \\
\text { deaer }^{c}\end{array}$ & $\begin{array}{c}\Phi \\
\text { aerated }^{c}\end{array}$ & $\begin{array}{l}\tau(\mu s) \\
\text { deaer }\end{array}$ & $\begin{array}{c}\tau(\mu \mathrm{s}) \\
\text { aerated }\end{array}$ & $\begin{array}{c}k_{r} \\
\left(10^{5}\right. \\
\left.\mathrm{s}^{-1}\right)\end{array}$ & $\begin{array}{c}k_{n r} \\
\left(10^{5}\right. \\
\left.\mathrm{s}^{-1}\right)\end{array}$ & $\begin{array}{c}\lambda_{\max } \\
(\mathrm{nm})^{b}\end{array}$ & $\begin{array}{c}\tau \\
(\mu s)\end{array}$ \\
\hline $\mathrm{Ru}-\mathrm{NH}_{2}$ & 620 & $0.023^{\mathrm{d}}$ & $0.0089^{\mathrm{d}}$ & 1.2 & 0.155 & 0.194 & 8.13 & 588 & 6.4 \\
\hline $\mathbf{R u}($ bpy $) 3^{\mathrm{f}}$ & 621 & 0.06 & 0.016 & 0.6 & 0.117 & 10 & 165.6 & 579 & 5.7 \\
\hline
\end{tabular}

${ }^{a}$ All data for complexes in $\mathrm{CH}_{3} \mathrm{CN}$, except otherwise specified. ${ }^{b} \lambda_{\text {ex }}=450 \mathrm{~nm} .{ }^{c}$ Quantum yields $\mathrm{d}(\Phi)$ are measured versus $\mathrm{Ru}(\mathrm{bpy})_{3}$ in aerated $\mathrm{CH}_{3} \mathrm{CN}(\Phi=0.016) .{ }^{e}$ In butyronitrile glass matrix. fRef.

Table 3.3: Luminescence data of $\mathrm{Ru}-\mathrm{NH}_{2}$ and Irbpy at RT as well as at $77 \mathrm{~K}$. 


\section{Electrochemistry of Ru- $\mathrm{NH}_{2}$}

$\mathrm{Ru}(\mathrm{II})$ complexes exhibit a very rich redox electrochemistry. The oxidation is associated with the central metal ion, while the reduction processes are mainly associated with the ligands coordinated to the metal center in an octahedral coordination sphere. $\mathbf{R u}(\mathbf{b p y})_{3}$, the reference compound shows highly reversible electrochemical behavior. It is characterized by one monoelectronic oxidation at $0.89 \mathrm{~V}$ vs. ferrocene/ferrocene ${ }^{+}$and three monoelectronic reduction peaks at -1.72 $\mathrm{V},-1.95 \mathrm{~V}$ and $-2.26 \mathrm{~V}$ respectively. ${ }^{76}$ The oxidation is assigned to the metal center whereas the three reduction peaks are ascribed to the reduction of each of the bpy ligands. $\mathbf{R u}-\mathbf{N H}_{2}$ displays similar electrochemical behavior to that of the reference compound. However, $\mathbf{R u}-\mathbf{N H}_{2}$, unlike the reference compound, exhibits two oxidation peaks at $0.6 \mathrm{~V}$ and $0.89 \mathrm{~V}$ respectively versus ferrocene/ferrocene ${ }^{+}$redox couple as shown in Figure 3.11. The first oxidation peak is not reversible and arises possibly from the oxidation of the amino group whereas the second is reversible and attributed to one electron oxidation of the metal center. Similar to $\mathbf{R u}(\mathbf{b p y})_{3}, \mathbf{R u}-\mathbf{N H}_{2}$ exhibits 3 reversible reduction peaks owing to successive reduction of three ligands coordinated to $\mathrm{Ru}^{2+}$ metal center at $-1.77 \mathrm{~V},-1.9 \mathrm{~V}$ and $-2.1 \mathrm{~V}$ respectively. 


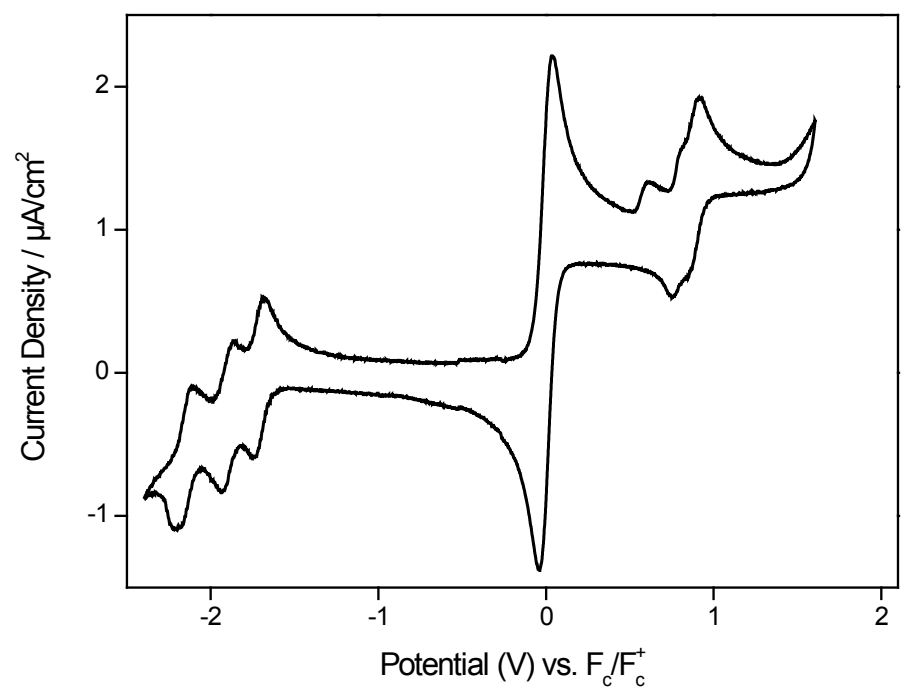

3.11: Cyclic voltammogram of $\mathbf{R u}-\mathbf{N H}_{2}$ in neat acetonitrile solution measured against ferrocene/ferrocene ${ }^{+}$redox couple.

\section{$\mathrm{CdSe} / \mathrm{ZnS}$ quantum dots}

In the present study, commercially available $\mathbf{C d S e} / \mathrm{ZnS}$ core-shell quantum dots (Lake Placid Blue CdSe/ZnS Core Shell Evidots, Evident technologies) have been employed. The photophysical properties of these nanocrystals are shown in Figure 3.12. The diameter of the nanocrystal is estimated from the absorption coefficient by the method reported by $\mathrm{Yu}$ and coworkers. For the particles used here, the core size is estimated to be about $1.9 \mathrm{~nm}$ which excludes the capping ligands. ${ }^{65}$ These core-shell nanocrystals are highly stable upon exposure to oxygen atmosphere and are extremely photostable. These nanoparticles were provided by the company as a suspension in toluene with TOPO and other aromatic hydrocarbon capping ligands. For these nanocrystals the emission maximum lies very close to the absorption onset indicating that the emission occurs upon direct recombination of the charge carriers. ${ }^{21}$ For the quantum dots used in the present study, the band edge 
absorption is at $463 \mathrm{~nm}$ with a molar absorptivity coefficient of about $42000 \mathrm{dm}^{3} \mathrm{~mol}^{-1} \mathrm{~cm}^{-1}$. The band edge luminescence is at $485 \mathrm{~nm}$. The quantum yield of the batch of dots was estimated to be about 0.4 measured by using fluorescein as the reference.

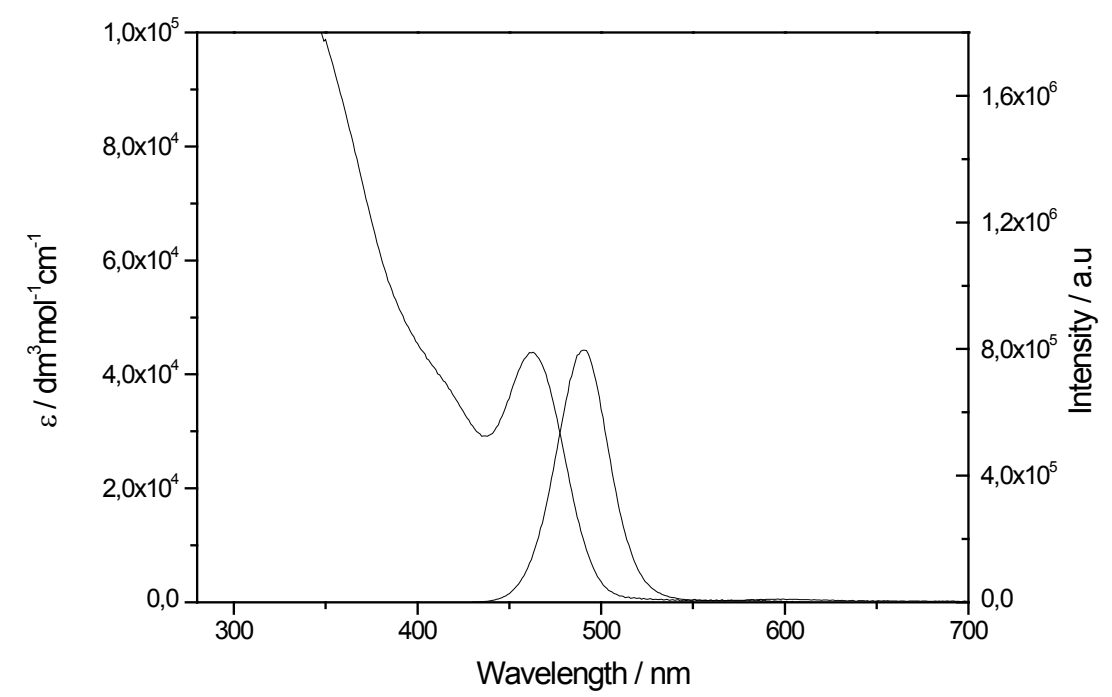

3.12: Absorption and emission spectra of $\mathrm{CdSe} / \mathrm{ZnS}$ quantum dots in toluene.

$$
\lambda_{\mathrm{ex}}=402 \mathrm{~nm} \text {. }
$$

\section{Functionalization of $\mathrm{CdSe} / \mathrm{ZnS}$ with $\mathrm{Ru}-\mathrm{NH}_{2}$}

$\mathbf{R u}-\mathbf{N H}_{2}$ was used to functionalize blue emitting $\mathbf{C d S e} / \mathrm{ZnS}$ quantum dots which are commercially available. The $\mathrm{ZnS}$ layer has a poor binding affinity to the amine moiety. Hence in order to enhance the binding ability, a different ligand exchange strategy was employed. Here a ligand exchange strategy reported by Dubois and coworkers by using carbon disulfide was employed to coordinate the ruthenium complex through a dithiocarbamate moiety. ${ }^{77}$ The simple ligand exchange strategy is presented in Scheme 3.3. Briefly, 35 nmoles of quantum dots from the stock solution were washed by precipitating with $5 \mathrm{~mL}$ methanol. The 
suspension was centrifuged at $13200 \mathrm{rpm}$ for 20 minutes. The supernatant was discarded and the precipitate was dispersed in $1 \mathrm{~mL}$ chloroform $\left(\mathrm{CHCl}_{3}\right)$.

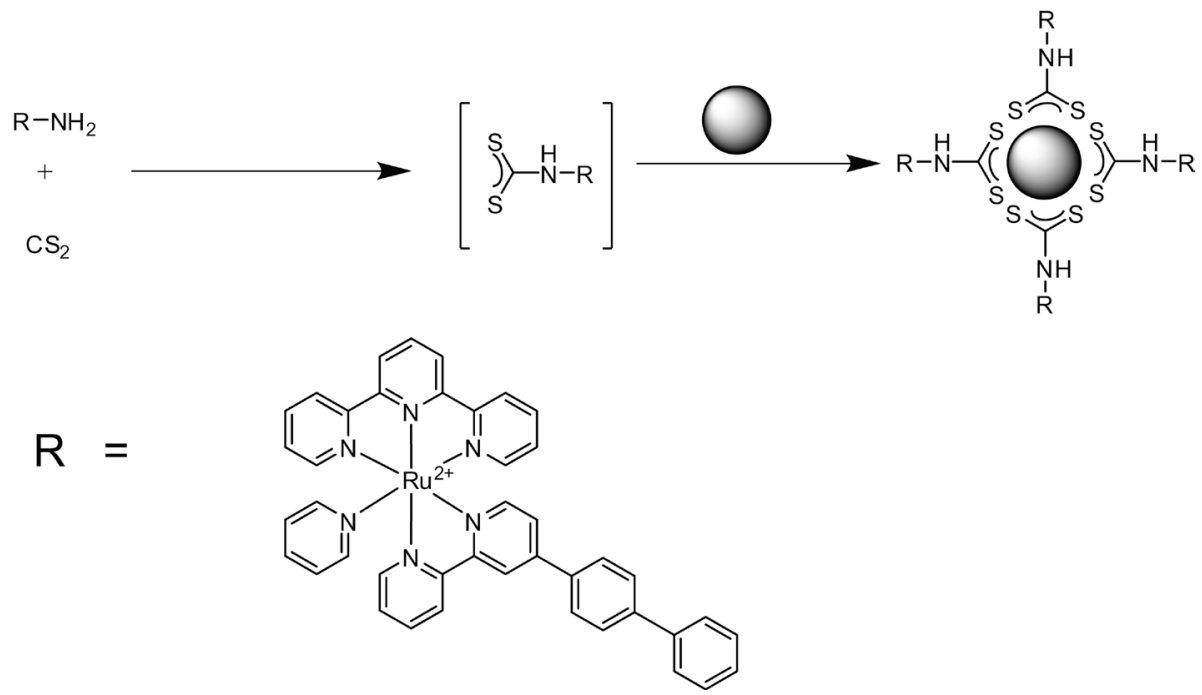

Scheme 3.3: Carbon disulfide mediated ligand exchange strategy

To this $1.2 \mathrm{mg}(1 \mu \mathrm{mol})$ of $\mathbf{R u}-\mathbf{N H}_{2}$ was added followed by $2.4 \mu \mathrm{L}$ of $\mathrm{CS}_{2}(2 \mu \mathrm{mol})$. The mixture was stirred overnight at room temperature. The quantum dots were washed again with methanol. Unbound $\mathrm{Ru}-\mathrm{NH}_{2}$ was soluble in methanol and the quantum dots coordinated to $\mathbf{R u}-\mathbf{N H}_{2}$ were precipitated. The metal complex coated nanocrystals were redispersed in toluene. However, the solubility of quantum dots was reduced upon coordination to a great extent in non polar solvents like toluene, hexane and chloroform in which quantum dots are usually very well soluble. Hence a mixture of solvents was used for further measurements. The change in solubility was an indirect evidence of modification of the nanocrystal surface with ruthenium complex. The number of $\mathbf{R u}-\mathbf{N H}_{2}$ complexes coordinated to the dot surface was estimated by absorption measurements. 


\section{Estimation of number of complexes coordinated to the quantum dot surface}

A stock solution of ruthenium complex $(47.9 \mu \mathrm{M})$ was prepared in a mixture of 4:1 v/v chloroform and acetonitrile. This solution was divided into two parts. To one part, $100 \mu \mathrm{L}$ of quantum dot solution (12 $\mathrm{nmol}$ ) was added followed by $2 \mu \mathrm{L}$ of $\mathrm{CS}_{2}$ solution and $2.2 \mathrm{~mL}$ of methanol were used to precipitate the quantum dots. The mixture was centrifuged and the absorption spectrum of the supernatant was measured. To the other reference solution $100 \mu \mathrm{L}$ of solvent was added (instead of quantum dots) and $2 \mu \mathrm{L}$ of $\mathrm{CS}_{2}$ solution and $2.2 \mathrm{~mL}$ of methanol were added and the absorption of this mixture was measured. Since the molar absorptivity coefficient of $\mathbf{R u}-\mathbf{N H}_{2}$ was known, the number of ruthenium complexes coordinated to the quantum dots was calculated from the difference in absorbance between the two solutions (Figure 3.13),. For the batch of quantum dots used, it was calculated that for $12 \mathrm{nmol}$ of quantum dots used, about $6 \mathrm{nmol}$ of $\mathbf{R u}-\mathbf{N H}_{2}$ were bound to the nanocrystals. In other words, for every two quantum dots in the solution, about one complex is bound.

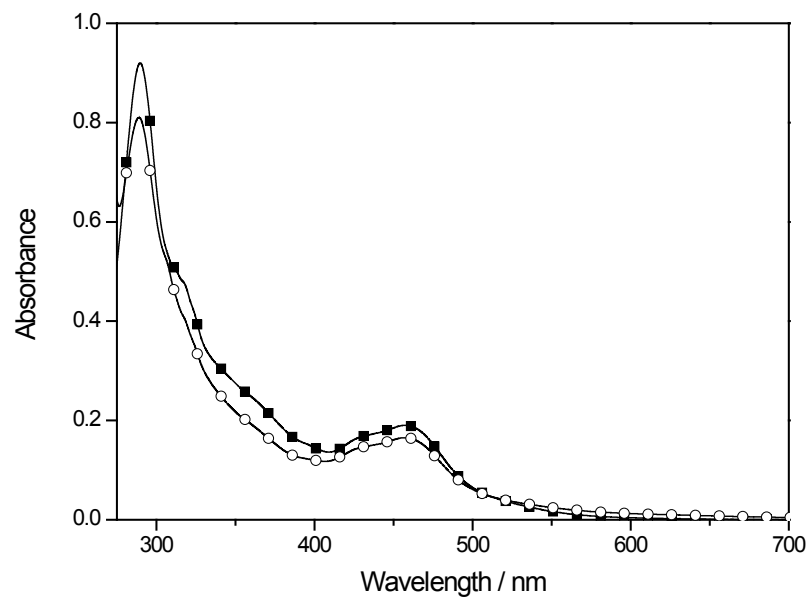

3.13: Absorption of $\mathbf{R u}-\mathbf{N H}_{\mathbf{2}}$ before (--) and after (-०-) uptake from quantum dots. 


\section{Energy transfer studies in CdSe/ $\mathrm{ZnS}-\mathrm{Ru}-\mathrm{NH}_{2}$ nanoassemblies}

The optical properties of quantum dots depend to a certain extent on the nature of capping ligands used. Conventional synthesis of quantum dots yields them with capping ligands like TOPO, dodecylamine or hexadecylamine which renders the dots soluble in apolar solvents such as toluene, hexane or chloroform. ${ }^{25}$ However, various ligand exchange strategies could be employed to systematically vary the capping ligands and thus control the properties of the quantum dots like solubility and luminescence. The choice of the quantum dots used for the present study was based on the reasonably good spectral overlap between the quantum dot (donor) emission and ruthenium complex (acceptor) absorption which is presented in Figure 3.14. For energy transfer experiments the solutions were prepared in such a way that the concentration of both components in the reference and assembly were identical. In general, the ratio of quantum dots to the ruthenium complex was maintained at 1:1 as it was determined before that approximately for every two dots, one complex would be bound. Since the concentration used for the measurements was very low the QD-Ru assembly could not be isolated at these concentrations.

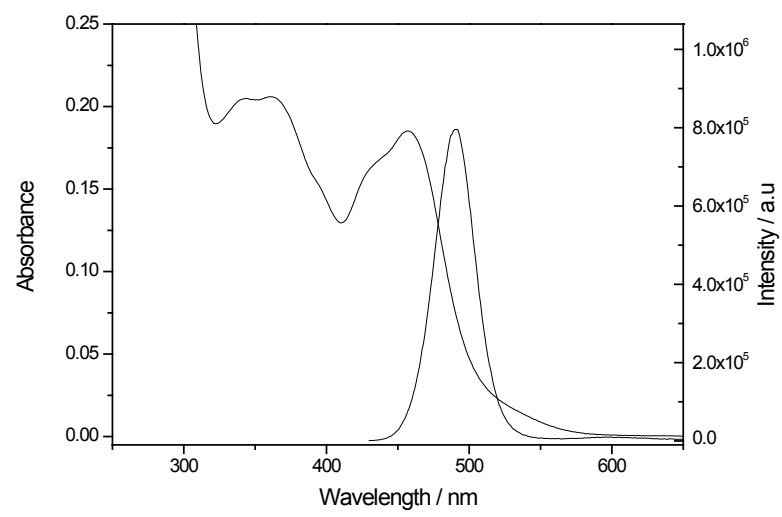

Figure 3.14: Spectral overlap between $\mathbf{C d S e} / \mathrm{ZnS}$ (donor) and $\mathbf{R u}-\mathbf{N H}_{2}$ (acceptor) 
Absorption spectra of quantum dot - ruthenium assemblies along with that of corresponding reference solutions are presented in Figure 3.15. The ${ }^{1}$ MLCT band of $\mathbf{R u}-\mathbf{N H}_{2}$ undergoes a hypsochromic shift of $3 \mathrm{~nm}$ indicating interaction between the two chromophores in the ground state.

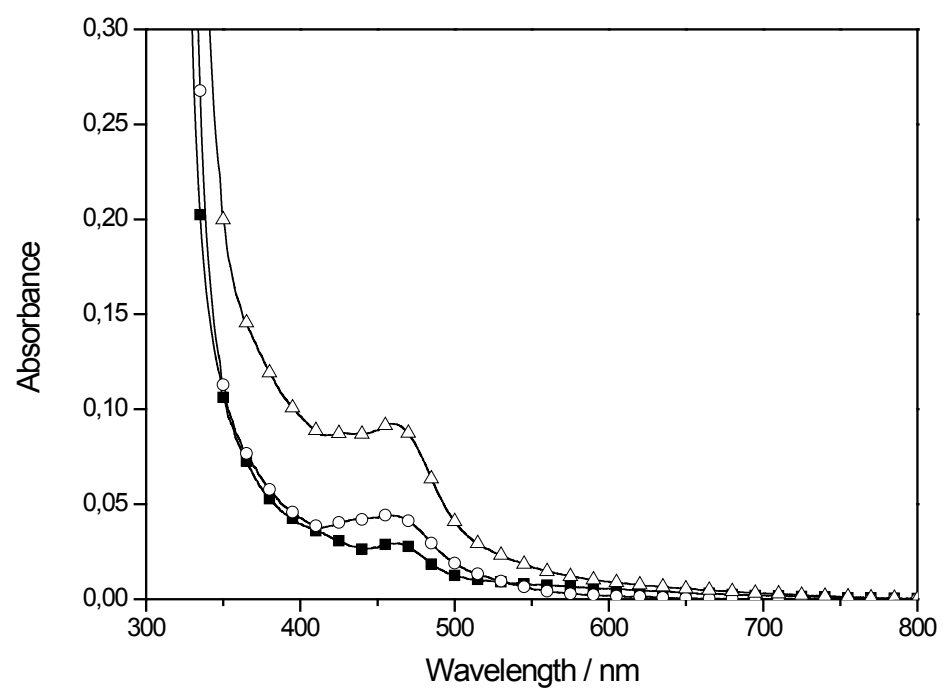

Figure 3.15: Absorption spectra of $\mathbf{C d S e} / \mathbf{Z n S}(--), \mathbf{R u}-\mathbf{N H}_{2}\left(-_{-}^{-}\right)$and $\mathbf{C d S e} / \mathbf{Z n S} / \mathbf{R u}$ assembly $(-\Delta-)$ in $1: 1 \mathrm{v} / \mathrm{v}$ mixture of methanol/toluene 


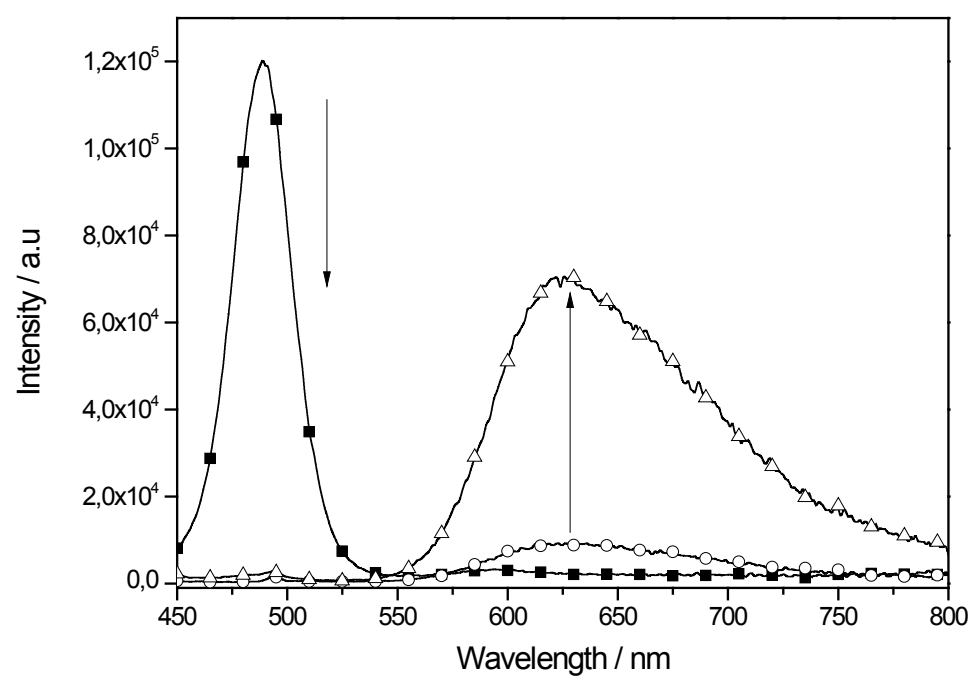

Figure 3.16: Room temperature emission spectra of CdSe/ZnS (--), Ru-NH $\mathbf{N H}_{2}$ $\left(-_{--}\right)$and $\mathbf{C d S e} / \mathbf{Z n S} / \mathbf{R u}$ assembly (- - -) in 1:1 v/v mixture of methanol/toluene. $\lambda_{\text {ex }}=431 \mathrm{~nm}$.

Room temperature emission spectra of the $\mathbf{C d S e} / \mathbf{Z n S} / \mathbf{R u}$ assembly in a solvent mixture of 1:1 v/v methanol/toluene along with corresponding reference solutions are presented in Figure 3.16. Upon excitation at 431 $\mathrm{nm}, \mathbf{C d S e} / \mathrm{ZnS}$ quantum dots exhibit an intense emission band that arises from radiative recombination of excitons at $483 \mathrm{~nm}$. $\mathbf{R u}-\mathbf{N H}_{2}$ shows a broad structureless band that originates from ${ }^{3} \mathrm{MLCT}$ emission. In the quantum dot - ruthenium assembly, the nanocrystal emission gets drastically quenched. This is accompanied by a complementary rise in the ruthenium emission displaying a sensitization of ruthenium complex by the semiconductor quantum dots. To further examine the photoinduced energy transfer process the excited state lifetimes of the assembly as well as the individual components were measured using a time correlated single photon counting (TCSPC) system. The present batch of quantum dots exhibits normally a triexponential decay of their excited state 
lifetimes. Upon washing with methanol, certain amounts of capping ligands are washed away thereby creating some vacant sites on the nanocrystal surface. This creates some defect states which contributes a component to the excited state decay. The measured lifetimes for the CdSe/ZnS quantum dots were 4 ns $(22 \%), 15$ ns $(56 \%)$ and 53 ns $(22 \%)$. The average lifetime for these nanocrystals is $\langle\tau\rangle=20.94$ ns. The $4 \mathrm{~ns}$ and 53 ns components are possibly due to the surface sites, whereas the $15 \mathrm{~ns}$ is the main component arising from the excitonic recombination. ${ }^{78}$ However, the lifetime of the quantum dots coordinated to the ruthenium complex shows biexponential decay with quenched lifetimes for the quantum dots as shown in Figure 3.17.

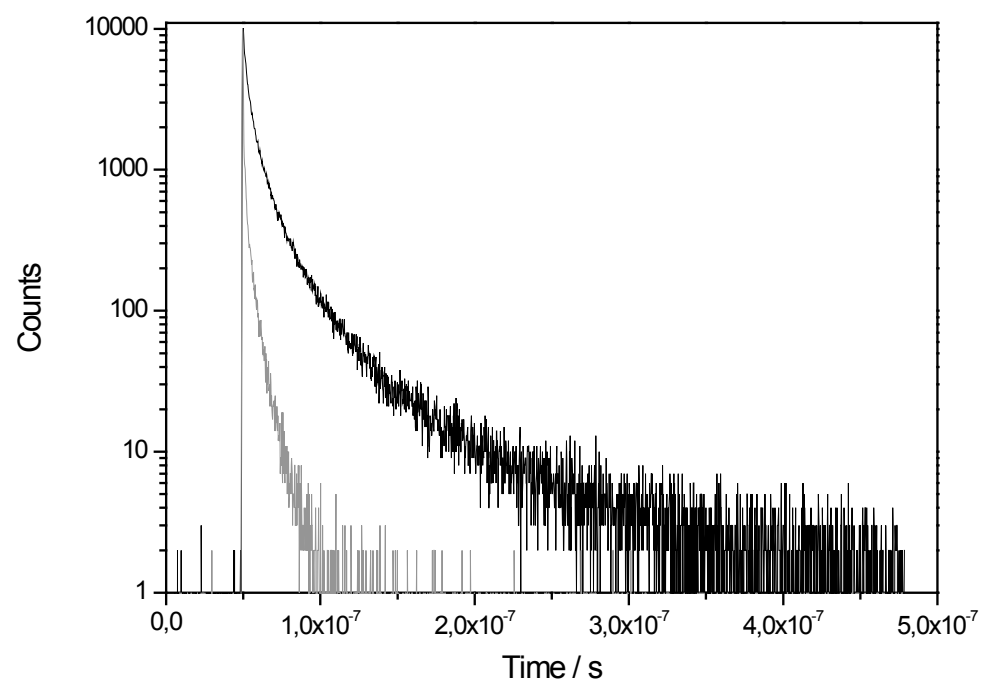

Figure 3.17: Excited state lifetimes of free quantum dots (black curve) and Ru$\mathrm{NH}_{2}$ functionalized quantum dots (grey curve), monitored at $484 \mathrm{~nm}$. $\lambda_{\text {ex }}=431$ $\mathrm{nm}$. 
In the assembly, the QD lifetime was quenched to 6 ns (85\%) and 4 ns $(15 \%)$ respectrively giving an average lifetime of 5.7 ns. By using equation 3.1, the energy transfer efficiency was calculated to be about $72 \%$.

In order to rule out any long range interaction, a control experiment was carried out in which the ruthenium complex $\mathbf{R u} \mathbf{u}(\mathbf{b p y})_{3} \mathbf{p h}_{4}$ was used with quantum dots under identical conditions as those used for CdSe/ZnS/Ru assemblies. This complex lacks any terminal group that could coordinate to the dot surface and the spectral overlap with the nanocrystals was similar. This ruthenium complex quenched the quantum dot emission as shown in Figure 3.18. However, the lifetime of quantum dots was not shortened indicating a static quenching mechanism wherein the quantum dot and ruthenium complex form a weak complex in the ground state which is non emissive. This weak interaction of the complexes and quantum dots can be explained based on electrostatic interactions. The quantum dots have a negatively charged surface as revealed by the zeta potential measurements $(-28 \mathrm{~V}$ measured in toluene for the batch of nanocrystals used in this study) and the ruthenium core has a positive charge. In absence of any terminal binding groups, there can be a random orientation of ruthenium molecule around the nanocrystal surface thereby giving a weakly bound ground state complex governed by electrostatic interactions. In addition, there was no sensitization of ruthenium complexes despite good spectral overlap and thus eliminating any possibility of a radiative or a 'trivial' energy transfer. From this control experiment it was clear that the sensitization observed for $\mathbf{R u}-\mathbf{N H}_{2}$ is indeed a photoinduced energy transfer. More detailed explanation about quenching mechanism is presented in the next section. 


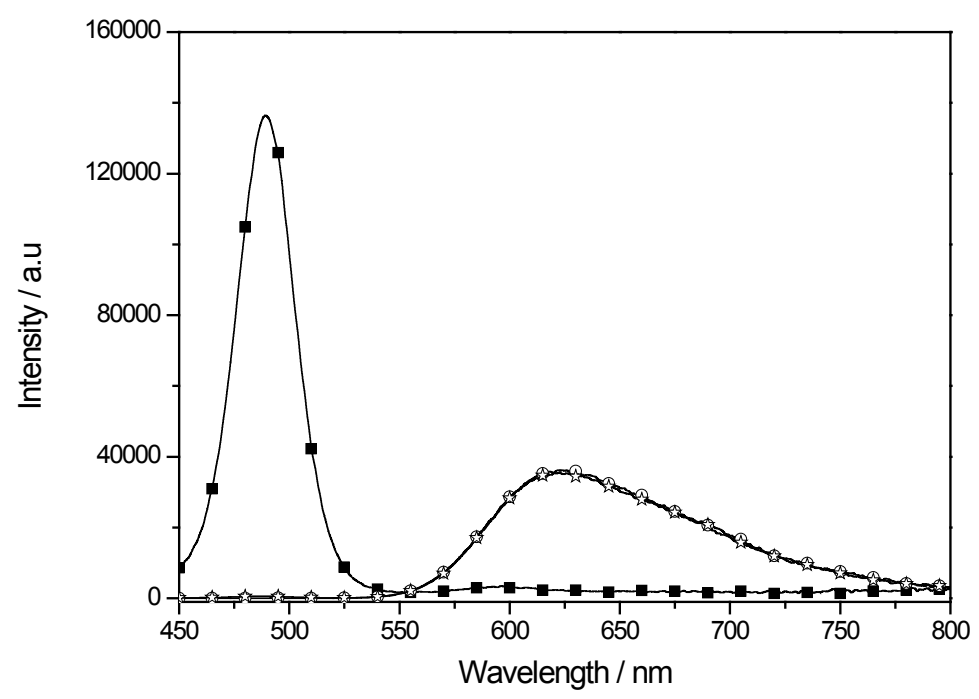

Figure 3.18: Room temperature emission spectra of CdSe/ZnS (--), $\mathbf{R u}\left(\right.$ bpy) $3-\mathrm{ph}_{4}\left(\right.$ (-o- $^{-}$and QD-Ru mixture (- $\boldsymbol{\varkappa}_{-}$) in 1:1 v/v mixture of methanol/toluene. $\lambda_{\text {ex }}=431 \mathrm{~nm}$.

\section{Förster Resonance Energy Transfer (FRET) discussion}

The observed photoinduced energy transfer mechanism in both the above systems could follow either an electron exchange mechanism (Dexter type) or through columbic or induced dipole interactions (Förster type). The prerequisite for energy transfer through electron exchange mechanism is that the donor and acceptor molecules should be in very close to each other such that there is sufficient orbital overlap between the donor and the acceptor. The orbital overlap decreases rapidly as the distance between the donor and acceptor increases. The energy transfer rate in the Dexter ${ }^{79}$ type mechanism decreases as $\exp (-$ $2 \mathrm{R} / \mathrm{L}$ ), where $\mathrm{R}$ is the donor acceptor separation relative to their van der Waals radii $\mathrm{L}$. Hence the energy transfer rate approaches negligibly small values as the donor acceptor distance increase to more than a couple of 


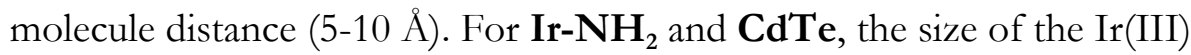
core can be roughly estimated to be about $1 \mathrm{~nm}$ and the two phenyl rings and the amine group would add roughly another $1 \mathrm{~nm}$. The capping ligands around the quantum dots could also increase the distance between the donor and acceptor. From the absorption spectrum of the $\mathbf{C d T e} / \mathbf{I r}-\mathbf{N H}_{2}$ assembly it was evident that the donor and acceptor do not interact in the ground state. Hence assuming a relatively weaker coordination which would decrease an orbital overlap, the donor acceptor separation could be approximated to be more than $2 \mathrm{~nm}$ which is too large for the Dexter mechanism to be operative. In case of Ru$\mathbf{N H}_{2}$ and $\mathbf{C d S e} / \mathrm{ZnS}$ quantum dots, the distance between QD and ruthenium core is separated by two rigid phenyl moieties and a dithiocarbamato group. In addition the CdSe core has a few layers of $\mathrm{ZnS}$ shell around it and some capping ligands coordinated to the surface. All these factors would contribute to the increase in donor acceptor distance and hence decrease the orbital overlap. Further, the Dexter mechanism takes place through electron exchange. The shell of large band gap ZnS around CdSe core would render electron transfer impossible. From these observations, we could safely say that energy transfer does not follow a Dexter mechanism.

The conditions necessary for the photoinduced energy transfer to be through Förster ${ }^{80-82}$ type are (i) a good spectral overlap between the donor and acceptor, (ii) large radiative rate constant for the donor, (iii)large molar extinction coefficient of the acceptor and (iv) small donor acceptor separation and the appropriate relative orientation between their electronic transition dipoles. For both $\mathbf{C d T e} / \mathbf{I r}-\mathbf{N H}_{2}$ and $\mathbf{C d S e} / \mathbf{Z n S} / \mathbf{R u}$ systems, all the above conditions are satisfied. To be specific, from Figure 3.4 and 3.12, it is evident that there is a good spectral overlap between the respective donor and the acceptor systems which would result in a large value of spectral overlap integral $J$. The radiative rate constant for the quantum dots is of the order of $10^{7}$. The molar extinction coefficient of ruthenium acceptor is of the order of $10^{4}$ whereas for the CdTe acceptor it is around $10^{5}$. The donor acceptor separation (distance between center to center of the electronic transition 
dipoles) is relatively small (approximately $2-3 \mathrm{~nm}$ as estimated from the size of the two complexes, the two phenyl ring spacers and assuming that the quantum dots are spherical). Hence we can quantify the Förster resonance energy transfer (FRET) between QDs and metal complexes. A detailed description of FRET can be found in the introductory chapter of this thesis.

The Förster radius, $R_{0}$ was calculated using the equation 1.3

$$
R_{0}^{6}=\frac{9000(\ln 10) \kappa^{2} Q_{D}}{128 \pi^{5} N n^{4}} \int_{0}^{\infty} F_{D}(\lambda) \varepsilon_{A}(\lambda) \lambda^{4} d \lambda
$$

where the integral term is the spectral overlap integral. These calculations were performed using a MATCAD program obtained from Prof. Gion Calzeferri. The donor-acceptor separation, r, could be estimated from the dependence of energy transfer efficiency on donor-acceptor distance and Förster radius as shown by the sigmoidal curve in figure 3.19 .

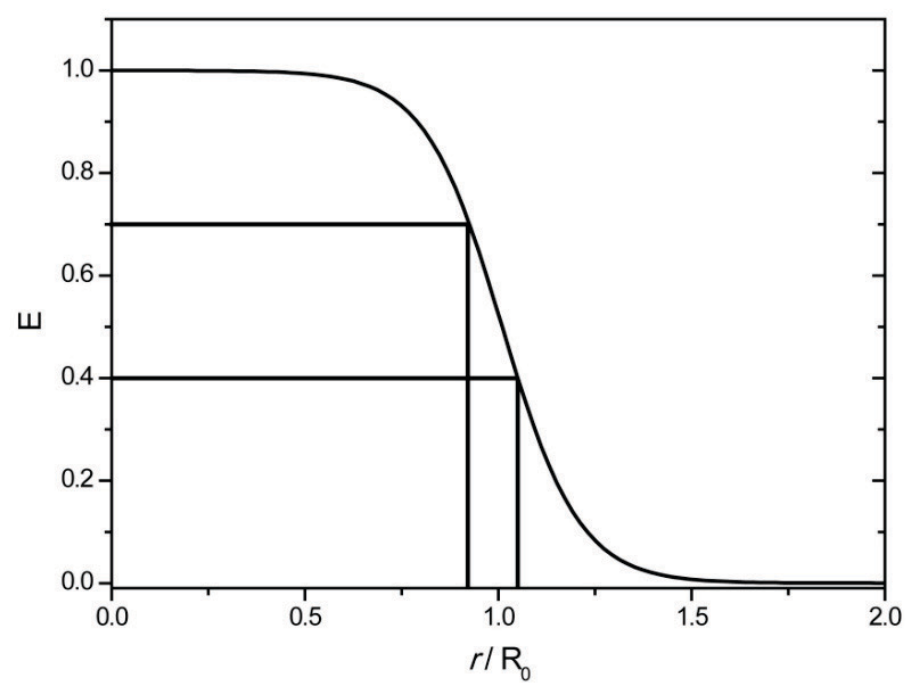

Figure 3.19: Dependence of energy transfer efficiency, $\mathrm{E}$ on distance. $\mathrm{R}_{0}$ is the Förster radius. ${ }^{83}$ 
In case of $\mathbf{C d T e} / \mathbf{I r}-\mathbf{N H}_{2}$ the Förster radius was calculated to be $\mathrm{R}_{0}=$ $35.52 \AA$, and the spectral overlap integral, $J=7.054 \times 10^{-10} \mathrm{~cm}^{6} / \mathrm{mol}$. From the quenching of the donor lifetime and the sensitization of the acceptor, the efficiency of energy transfer was estimated to be about $40 \%$. Using this value in figure 3.17 , the $r / R_{0}$ turns out to be 1.05 , and hence the donor-acceptor separation, $\mathrm{r}=37.296 \AA$. The energy transfer rate is calculated by using the equation 1.4 ,

$$
k_{T}(r)=\frac{1}{\tau_{D}}\left(\frac{R_{0}}{r}\right)^{6}
$$

Hence using all the above values and $\tau_{\mathrm{D}}$, the lifetime of the donor as 1.4 $\mu$ s in the above equation, the calculated energy transfer rate is $k_{T}=5.33$ x $10^{5} \mathrm{~s}^{-1}$.

For the $\mathbf{C d S e} / \mathrm{ZnS} / \mathbf{R u}$ system, the energy transfer parameters such as the Förster radius, $\mathrm{R}_{0}$, and the spectral overlap integral, $J$ were calculated to be $\mathrm{R}_{0}=36.428 \AA$, and $J=4.382 \times 10^{-11} \mathrm{~cm}^{6} / \mathrm{mol}$. From the emission and lifetime measurements, we can estimate the energy transfer efficiency to be around $70 \%$. From this we can estimate that the donoracceptor separation is lower than the Förster distance. From this plot, we can approximate $r$ to be about $0.92 \mathrm{R}_{0}$ which is about $33.51 \AA$. Incorporating all these values and $\tau_{\mathrm{D}}$ the lifetime of the donor, in this case the average lifetime of quantum dots as $20.94 \mathrm{~ns}$ in equation 3.4, the energy transfer rate is calculated to be $7.87 \times 10^{7} \mathrm{~s}^{-1}$.

\section{Conclusions}

In this chapter, synthesis of a cyclometalated $\operatorname{Ir}(\mathrm{III})$ complex and a $\mathrm{Ru}(\mathrm{II})$ polypyridyl complex both bearing a terminal amino group were described. A detailed photophysical and electrochemical study of these complexes were carried out and it was found that the Ir complex has a very low quantum efficiency and also irreversible redox behavior. Nonetheless, we have showed energy transfer from $\operatorname{Ir}(\mathrm{III})$ to QD through FRET with 40\% efficiency, which opens up the possibility to incorporate these assemblies in fabrication of OLEDs. The ruthenium 
complex on the other hand was found to have a better quantum yield and highly reversible electrochemical behavior. Further, this $\mathrm{Ru}(\mathrm{II})$ complex with terminal amine group was employed to functionalize $\mathrm{CdSe} / \mathrm{ZnS}$ quantum dots in the presence of $\mathrm{CS}_{2}$ in a one pot reaction. The number of ruthenium molecules that could coordinate to the quantum dot surface was estimated from the absorption measurements to be about one molecule per two nanocrystals. In such an assembly, a Förster Resonance Energy Transfer (FRET) was observed wherein the quantum dots act as energy donors and the ruthenium complex as the energy acceptors. The energy transfer was found out to be about $70 \%$ efficient and the Förster radius was calculated to be $36.428 \AA$. The spectral overlap was estimated to be $4.382 \times 10^{-11} \mathrm{~cm}^{6} / \mathrm{mol}$ and the energy transfer efficiency was calculated to be $1.77 \times 10^{8} \mathrm{~s}^{-1}$. Such an assembly can serve as a useful model system for photovoltaic applications where the quantum dots are predominantly employed as light harvesting species which could transmit the absorbed light energy to the photo-reactive center through an antenna mechanism.

\section{Synthesis of $\mathrm{Ir}_{2} \mathrm{Cl}_{2}$ (ppy)}

$1 \mathrm{~g}$ of Iridium trichloride hydrate $(3.3 \mathrm{mmol})$ and $1.91 \mathrm{~mL}$ of 2 phenylpyridine $(13.3 \mathrm{mmol})$ in a round bottomed flask were dissolved in $30 \mathrm{~mL}$ of 3:1 mixture of ethoxyethanol : water and refluxed for 24 hours. The solution was cooled to room temperature and the yellow precipitate was collected on a glass filter frit. The precipitate was washed with ethanol and acetone and then redissolved in dichloromethane and filtered. To the solution toluene $(75 \mathrm{~mL})$ and hexane $(30 \mathrm{~mL})$ and the resulting solution was reduced in volume under pressure to $50 \mathrm{~mL}$ and cooled to give crystals of $(1.2 \mathrm{~g})$. This was used as such for further reactions. 


\section{Synthesis of (bpy)(ph) ${ }_{2} \mathrm{NH}_{2}$}

Bpy-hh-Br (0.502 g, $1.6 \mathrm{mmol}), 4$-aminophenylboronic acid pinacol ester $0.388 \mathrm{~g}(1.8 \mathrm{mmol})$, tetrakis(triphenylphosphine)palladium(0) $(0.092$ g, $0.08 \mathrm{mmol})$ and Cesium carbonate $(0.577 \mathrm{~g}, 1.8 \mathrm{mmol})$ were put into $\mathrm{DMF}$ and heated to $100^{\circ} \mathrm{C}$ under nitrogen overnight. After cooling to RT the mixture was separated between water and dichloromethane. The combined organic phases were dried over magnesium sulfate and the solvent was removed under reduced pressure afterwards to yield $0.717 \mathrm{~g}$ of a brown precipitate. A flash column with a polarity gradient was used to purify the compound. Hexane:ethylacetate:triethylamine mixtures were used with the following ratios. 8:2:1 $(300 \mathrm{~mL}), 5: 2: 1(320 \mathrm{~mL})$ and 4:4:1 $(100 \mathrm{~mL})$. The fractions with the compound were combined and the solvents were removed under reduced pressure to give a dark yellow solid. The compound was dissolved in dichloromethane and washed with $2 \mathrm{M} \mathrm{NaOH}$ solution in water. The organic phase was dried, the solvent removed to yield a bright yellow compound. $270 \mathrm{mg}(52 \%)$

\section{Synthesis of $\left[\operatorname{Ir}(\mathrm{ppy})_{2}(\mathrm{bpy})(\mathrm{ph})_{2} \mathrm{NH}_{2}\right]^{+} \mathrm{PF}_{6}^{-}$}

$\mathrm{Ir}_{2} \mathrm{Cl}_{2}$ (ppy) $)_{4}(83 \mathrm{mg}, 0.522 \mathrm{mmol})$ and $44 \mathrm{mg}$ of bpy-bp- $\mathrm{NH}_{2}(0.157$ $\mathrm{mmol}$ ) in a round bottomed flask were dissolved in a solvent mixture of methanol and dichloromethane $(1: 3,20 \mathrm{~mL})$. The reaction mixture was refluxed for 3 hours under nitrogen atmosphere. The dichloromethane was evaporated under reduced pressure. To the resulting solution excess of methanolic ammoniumhexafluorophosphate was added. The resulting yellow precipitate was filtered and washed with water and ether. The impure compound was purified by column chromatography using silica gel in magic mixture (a mixture of $10 \% \mathrm{NaCl}$ solution in water, acetonitrile and methanol (1:6:1)). The resulting pure compound was an orange solid (105 mg). ${ }^{1} \mathrm{H}$ NMR (400 $\mathrm{MHz}, \mathrm{CD}_{3} \mathrm{CN}$ ) $\delta$ ppm: 8.77 (s, $2 \mathrm{H}), 8.08(\mathrm{tt}, 4 \mathrm{H}), 7.85(\mathrm{~m}, J=45.9,10 \mathrm{H}), 7.67(\mathrm{t}, 2 \mathrm{H}), 7.51(\mathrm{~d}, J=8.3$, $2 \mathrm{H}), 7.05 \mathrm{t}, 4 \mathrm{H}), 6.94(\mathrm{t}, 2 \mathrm{H}), 6.75(\mathrm{~d}, J=8.4,2 \mathrm{H}), 6.31(\mathrm{~d}, J=7.4,2 \mathrm{H})$, 4.39 (s, broad, $2 \mathrm{H})$. ESI-MS: $m / z=824.2(100 \%)$ 


\section{Synthesis of $\mathrm{Ru}(\mathrm{bpy})_{2} \mathrm{Cl}_{2} \cdot 2 \mathrm{H}_{2} \mathrm{O}$}

$\mathrm{RuCl}_{3} .3 \mathrm{H}_{2} \mathrm{O}$ (3.9 g, $14.54 \mathrm{mmol}$ ), bipyridine (4.68 g, $30.0 \mathrm{mmol}$ ), and $\mathrm{LiCl}(4.5 \mathrm{~g}, 100 \mathrm{mmol})$ were heated at reflux in reagent grade dimethylformamide $(25 \mathrm{~mL})$ for $8 \mathrm{~h}$. The reaction was stirred magnetically throughout this period. After the reaction mixture was cooled to room temperature, $125 \mathrm{~mL}$ of reagent grade acetone was added and the resultant solution cooled at $0^{\circ} \mathrm{C}$ overnight. The resulting product was filtered to yield a red-violet solution and a dark green-black microcrystalline product. The solid was washed three times with $25-\mathrm{mL}$ portions of water followed by three $25-\mathrm{mL}$ portions of diethyl ether, and then it was dried by suction. This yielded $4.5 \mathrm{~g}$ of $\mathrm{Ru}(\mathrm{bpy})_{2} \mathrm{Cl}_{2} \cdot 2 \mathrm{H}_{2} \mathrm{O}$. This product was used as such for further reactions.

\section{Synthesis of $\left[\mathrm{Ru}(\mathrm{bpy})_{2}(\mathrm{bpy})-\mathrm{ph}-\mathrm{Br}\right]^{2+} 2\left(\mathrm{PF}_{6}\right)^{-}$}

In a round bottomed flask, $0.160 \mathrm{~g}$ of $\mathrm{Ru}(\mathrm{bpy})_{2} \mathrm{Cl}_{2} \cdot 2 \mathrm{H}_{2} \mathrm{O}(0.307 \mathrm{mmol})$ and bpy-ph-Br ligand were mixed in $3.5 \mathrm{~mL}$ of ethyleneglycol. The mixture was sonicated for 10 minutes. Then the flask was transferred into a home built microwave reactor. The reaction was carried out for 5 minutes in short intervals of 1 minute. After every minute the reaction was monitored with TLC. Part of ethylene glycol was evaporated under reduced pressure. The residue was dissolved in $20 \mathrm{~mL}$ of water and extracted twice with $\mathrm{CHCl}_{3}(2 \times 20 \mathrm{~mL})$ until the violet colour of the residual complex disappeared. Any residual chloroform was removed by evaporation under reduced pressure. The product in the water layer was precipitated by adding excess of methanolic ammoniumhexafluorophosphate and the mixture was stirred for 5 minutes till the orange precipitate was obtained. The solid was filtered on celite and dried under vacuum to obtain fine powder. ${ }^{1} \mathrm{H}-\mathrm{NMR}(300$ $\left.\mathrm{MHz}, \mathrm{CD}_{3} \mathrm{CN}\right) \delta$ ppm: $8.60(\mathrm{~d}, 2 \mathrm{H}), 8.42(\mathrm{~d}, 4 \mathrm{H}), 7.96(\mathrm{~m}, 5 \mathrm{H}), 7.66(\mathrm{~m}$, $8 \mathrm{H}), 7.52(\mathrm{~m}, 3 \mathrm{H}), 7.30(\mathrm{~m}, 5 \mathrm{H})$ 


\section{Synthesis of $\left[\mathrm{Ru}(\mathrm{bpy})_{2}(\mathrm{bpy})-(\mathrm{ph})_{2}-\mathrm{NH}_{2}\right]^{2+} 2\left(\mathrm{PF}_{6}\right)^{-}$}

In a schlenk flask, $75 \mathrm{mg}$ of $\left[\mathrm{Ru}(\mathrm{bpy})_{2}(\mathrm{bpy})-\mathrm{ph}-\mathrm{Br}\right]^{2+} 2\left(\mathrm{PF}_{6}\right)^{-}(0.0739$ $\mathrm{mmol})$ and $80 \mathrm{mg}$ of $\mathrm{H}_{2} \mathrm{~N}-\mathrm{Ph}-\mathrm{BO}_{2} \mathrm{TME}(0.369 \mathrm{mmol})$ and $240 \mathrm{mg}$ of $\mathrm{Cs}_{2} \mathrm{CO}_{3}(0.739 \mathrm{mmol})$ were taken in $10 \mathrm{~mL}$ of dry DMF. The flask was repeatedly degassed for 3 cycles. Catalytic amount of $\mathrm{Pd}\left(\mathrm{PPh}_{3}\right)_{4}$ was added under steady flow of nitrogen and the reaction mixture was heated at $94^{\circ} \mathrm{C}$ for 20 hours. The DMF was removed under vacuum adding toluene. The solid was washed with ether. The solid was purified by column chromatography (silica) using magic mixture as an eluent (300 $\mathrm{mL}$ water, $300 \mathrm{~mL}$ methanol, $1200 \mathrm{~mL}$ acetonitrile and $32 \mathrm{~g} \mathrm{NaCl}$ ). The organic solvents were removed and the complex was precipitated adding ammonium hexafluorophosphate. The orange precipitate was filtered on celite, washed with water then with ether. The pure complex was reextracted using acetonitrile. The solvent was evaporated and finally, the solid was dried at $50^{\circ} \mathrm{C}$ for a night under vacuum to yield $65 \mathrm{mg}$ of pure $\left[\mathrm{Ru}(\mathrm{bpy})_{2}(\mathrm{bpy})-(\mathrm{ph})_{2}-\mathrm{NH}_{2}\right]^{2+} 2\left(\mathrm{PF}_{6}\right) \cdot{ }^{1} \mathrm{H}-\mathrm{NMR}\left(300 \mathrm{MHz}, \mathrm{CD}_{3} \mathrm{CN}\right) \delta$ ppm : 8.79 (d, $\left.{ }^{4} \mathrm{~J}=1.2 \mathrm{~Hz}, 1 \mathrm{H}\right), 8.72\left(\mathrm{~d},{ }^{3} \mathrm{~J}=8.1 \mathrm{~Hz}, 1 \mathrm{H}\right), 8.55(\mathrm{~m}, 4 \mathrm{H})$, $8.11(\mathrm{~m}, 5 \mathrm{H}), 7.93(\mathrm{~d}, 3 \mathrm{~J}=8.4 \mathrm{~Hz}, 2 \mathrm{H}), 7.78(\mathrm{~m}, 9 \mathrm{H}), 7.53$ (d, ${ }^{3} \mathrm{~J}=6.6 \mathrm{~Hz}$, 2H), $7.42\left(\mathrm{~d},{ }^{3} \mathrm{~J}=5.1 \mathrm{~Hz}, 5 \mathrm{H}\right), 6.76\left(\mathrm{~d},{ }^{3} \mathrm{~J}=8.4 \mathrm{~Hz}, 2 \mathrm{H}\right), 4.42$ (broad)

\section{Determination of concentration of quantum dot solution}

The accurate determination of quantum dots in a solution is extremely difficult. However, there are some empirical methods available to determine the concentration based on absorption measurements for different nanocrystals. In this chapter, the concentration of quantum dots was estimated following the procedure reported by $\mathrm{Yu}$ and coworkers. ${ }^{65}$ Briefly, $20 \mu \mathrm{L}$ of quantum dots from the stock solution was taken and made up to $5 \mu \mathrm{L}$ by adding toluene. The absorption spectrum of this solution was measured. According to the empirical method published by $\mathrm{Yu}$ and coworkers, for $\mathbf{C d T e}$ nanocrystals, the size, D is given by

$$
\mathrm{D}=\left(9.8127 \times 10^{-7}\right) \lambda^{3}-\left(1.7147 \times 10^{-3}\right) \lambda^{2}+(1.0064) \lambda-194.84
$$


where $\lambda$ is the exciton absorption maximum. Once the size is calculated, then the molar extinction coefficient is determined using the following expression.

$$
\varepsilon=10043(\mathrm{D})^{2.12}
$$

Upon knowing the epsilon value, it is possible to calculate the concentration of the quantum dots in the stock solution. It is noteworthy that the method reported above at time underestimates the size of the nanocrystals. In such cases, it is also pragmatic to use the values obtained from high resolution transmission electron microscopy (HRTEM).

CdTe/Ir(III) nanoassemblies: Dispersions of quantum dots and solutions of $\operatorname{Ir}(\mathrm{III})$ complexes were prepared inside the glove box. 250 $\mu \mathrm{L} \mathrm{CdTe} \mathrm{quantum} \mathrm{dots} \mathrm{from} \mathrm{the} \mathrm{raw} \mathrm{product}\left(4.2 \times 10^{-4} \mathrm{M}\right)$ were dissolved in $400 \mu \mathrm{L}$ toluene. To this was added $3 \mathrm{~mL}$ of methanol to precipitate the quantum dots and subsequently centrifuged. The supernatant was discarded and the residue was dissolved in $10 \mathrm{~mL}$ of dichloromethane and further diluted ten times to obtain $\sim 10^{-6} \mathrm{M}$ quantum dot stock solution. A $10^{-5} \mathrm{M}$ stock solution of $\operatorname{Ir}(\mathrm{III})$ complexes (Ir-NH $\mathbf{N H}_{2}$ and $\mathbf{I r}-\mathbf{b} \mathbf{p}$ ) was prepared in the same solvent. In general, both the solutions were mixed in such a way that the ratio of CdTe to $\operatorname{Ir}(\mathrm{III})$ was 1:10 and their respective concentrations $10^{-6} \mathrm{M}$ and $10^{-5} \mathrm{M}$ allowed to stand for $\sim 1$ hour in order to ensure that the iridium complex would displace a sufficient number of capping ligands from the QD surface. All the reference systems, $\mathbf{I r}-\mathbf{N H}_{\mathbf{2}} / \mathbf{I r}-\mathbf{b p}, \mathbf{C d T e}$ as well as the 1:10 mixture were prepared in such a way that the concentrations of the chromophores in reference and the complex solutions were same.

Photophysics: Absorption spectra were measured on a Varian Cary 5000 double-beam UV-Vis-NIR spectrometer and baseline corrected. Steady-state emission spectra were recorded on a HORIBA Jobin-Yvon IBH FL-322 Fluorolog 3 spectrometer equipped with a $450 \mathrm{~W}$ xenon arc lamp, double grating excitation and emission monochromators (2.1 $\mathrm{nm} / \mathrm{mm}$ dispersion; 1200 grooves $/ \mathrm{mm}$ ) and a Hamamatsu R928 
photomultiplier tube or a TBX-4-X single-photon-counting detector. Emission spectra were corrected for source intensity (lamp and grating) and emission spectral response (detector and grating) by standard correction curves. Time-resolved measurements were performed using the time-correlated single-photon counting (TCSPC) option on the Fluorolog 3. NanoLED (402 nm; FWHM < 750 ps) with repetition rates between $10 \mathrm{kHz}$ and $1 \mathrm{MHz}$ were used to excite the sample. The excitation sources were mounted directly on the sample chamber at $90^{\circ}$ to a double grating emission monochromator $(2.1 \mathrm{~nm} / \mathrm{mm}$ dispersion; 1200 grooves $/ \mathrm{mm}$ ) and collected by a TBX-4-X single-photon-counting detector. The photons collected at the detector are correlated by a timeto-amplitude converter (TAC) to the excitation pulse. Signals were collected using an IBH DataStation Hub photon counting module and data analysis was performed using the commercially available DAS6 software (HORIBA Jobin Yvon IBH). The quality of fit was assessed by minimizing the reduced chi squared function $\left(\chi^{2}\right)$ and visual inspection of the weighted residuals. 


\section{References}

1. Montalti, M.; Credi, A.; Prodi, L.; Gandolfi, M. T., Handbook of Photochemistry. 3rd ed.; Taylor \& Francis: 2006.

2. Roundhill, D. M., Photochemistry and Photophysics of Metal Complexes. Plenum Press: New York, 1994.

3. Campagna, S.; Puntoriero, F.; Nastasi, F.; Bergamini, G.; Balzani, V., Top. Curr. Chem. 2007, $280,117$.

4. Flamigni, L.; Barbieri, A.; Sabatini, C.; Ventura, B.; Barigelletti, F., Top. Curr. Chem. 2007, 281, 143.

5. Balzani, V., Tetrahedron 1992, 48, 10443.

6. Balzani, V.; De Cola, L., Supramolecular Chemistry. Kluwer: Dordrecht, 1992.

7. Balzani, V.; Juris, A.; Venturi, M.; Campagna, S.; Serroni, S., Chem. Rev. 1996, 96, 759.

8. Balzani, V.; Moggi, L.; Scandola, F., Supramolecualr Photochemistry. Kluwer: Reidel: Dordrecht, 1987.

9. De Cola, L.; Belser, P., Coord. Chem. Rev. 1998, 177, 301.

10. Dixon, I. M.; Collin, J.-P.; Sauvage, J.-P.; Flamigni, L.; Encinas, S.; Barigelletti, F., Chem. Soc. Rev. 2000, 29, 385.

11. Scandola, F.; Indelli, M.; Chiorboli, C.; Bignozzi, C., Photoinduced electron and energy transfer in polynuclear complexes. In Photoinduced Electron Transfer II, 1990; pp 73.

12. Watts, R. J., Comments Inorg. Chem. 1991, 11, 303

13. Manfred, J. W.; John, M. L., Spin Correlations in Organic LightEmitting Diodes. In Highly Efficient OLEDs with Phosphorescent Materials, Prof. Dr. Hartmut, Y., Ed. 2008; pp 99.

14. Yersin, H., In Highly Efficient OLEDs with Phosphorescent Materials Wiley VCH: Berlin, 2007.

15. Adachi, C.; Kawamura, Y.; Goushi, K.; Endo, A., Oyo Butsuri 2006, 75, 1465.

16. Pi-Tai, C.; Yun, C., Chem. Eur. J. 2007, 13, 380.

17. Seo, S.; Inoue, H.; Ohsawa, N.; Shitagaki, S.; Nomura, R.; Yamazaki, S., Digest of Technical Papers - Society for Information Display International Symposium 2007, 38, 1776.

18. Thompson, M., MRS Bull. 2007, 32, 694. 
19. Bolink, H. J.; Cappelli, L.; Coronado, E.; Gratzel, M.; Orti, E.; Costa, R. D.; Viruela, P. M.; Nazeeruddin, M. K., J. Am. Chem. Soc. 2006, 128, 14786.

20. Costa, R. D.; Cespedes-Guirao, F. J.; Orti, E.; Bolink, H. J.; Gierschner, J.; Fernandez-Lazaro, F.; Sastre-Santos, A., Chem. Commun. 2009, 3886.

21. Alivisatos, A. P., J. Phys. Chem. 1996, 100, 13226.

22. Alivisatos, A. P., Science 1996, 271, 933.

23. Klimov, V. I., Semiconductor and Metal Nanocrystals. Synthesis and Electronic and Optical Properties. Marcel Dekker: New York, 2004; p 159

24. Nirmal, M.; Brus, L., Acc. Chem. Res. 1999, 32, 407.

25. Murray, C. B.; Kagan, C. R.; Bawendi, M. G., Annu. Rev. Mater. Sci. 2000, 545.

26. Daneshvar, H.; Nelms, J.; Muhammad, O.; Jackson, H.; Tkach, J.; Davros, W.; Peterson, T.; Vogelbaum, M. A.; Bruchez, M. P.; Toms, S. A., Nanomedicine 2008, 3, 21.

27. Hsieh, J.-M.; Ho, M.-L.; Wu, P.-W.; Chou, P.-T.; Tsai, T.-T.; Chi, Y., Chem. Commun. 2006, 615.

28. Callan, J.; De Silva, A.; Mulrooney, R.; Mc Caughan, B., J. Inclusion Phenom.Macrocyclic Chem. 2007, 58, 257.

29. Nazzal, A. Y.; Qu, L.; Peng, X.; Xiao, M., Nano Lett. 2003, 3, 819.

30. Klimov, V. I.; Mikhailovsky, A. A.; Xu, S.; Malko, A.; Hollingsworth, J. A.; Leatherdale, C. A.; Eisler, H. J.; Bawendi, M. G., Science 2000, 290, 314.

31. Wang, C.; Wehrenberg, B. L.; Woo, C. Y.; Guyot-Sionnest, P., J. Phys. Chem. B 2004, 108, 9027.

32. Colvin, V. L.; Schlamp, M. C.; Alivisatos, A. P., Nature 1994, 370 , 354.

33. Gur, I.; Fromer, N. A.; Geier, M. L.; Alivisatos, A. P., Science 2005, 310, 462.

34. Kongkanand, A.; Tvrdy, K.; Takechi, K.; Kuno, M.; Kamat, P. V., J. Am. Chem. Soc. 2008, 130, 4007. 
35. Leschkies, K. S.; Divakar, R.; Basu, J.; Enache-Pommer, E.; Boercker, J. E.; Carter, C. B.; Kortshagen, U. R.; Norris, D. J.; Aydil, E. S., Nano Lett. 2007, 7, 1793.

36. Lopez-Luke, T.; Wolcott, A.; Xu, L. p.; Chen, S.; Wen, Z.; Li, J.; De La Rosa, E.; Zhang, J. Z., J. Phys. Chem. C 2008, 112, 1282.

37. Munro, A. M.; Bardecker, J. A.; Liu, M. S.; Cheng, Y.-J.; Niu, Y.H.; Plante, I. J.-L.; Jen, A. K. Y.; Ginger, D. S., Microchim. Acta 2008, 160, 345.

38. Tessler, N.; Medvedev, V.; Kazes, M.; Kan, S.; Banin, U., Science 2002, 295, 1506.

39. Kamat, P. V., J. Phys. Chem. C 2008, 112, 18737.

40. Murray, C. B.; Norris, D. J.; Bawendi, M. G., J. Am. Chem. Soc. 1993, 115, 8706.

41. Nazzal, A. Y.; Qu, L.; Peng, X.; Xiao, M., Nano Letters 2003, 3, 819.

42. Xia;, Y.; Zhang;, T.; Diao; X.; Zhu., C., Chem. Lett. 2007, 36, 242.

43. Dabbousi, B. O.; Rodriguez-Viejo, J.; Mikulec, F. V.; Heine, J. R.; Mattoussi, H.; Ober, R.; Jensen, K. F.; Bawendi, M. G., J. Phys. Chem. B 1997, 101, 9463.

44. Hines, M. A.; Guyot-Sionnest, P., J. Phys. Chem. 1996, 100, 468.

45. Schaller, R. D.; Sykora, M.; Jeong, S.; Klimov, V. I., J. Phys. Chem. B 2006, 110, 25332.

46. Sykora, M. P., M. A.; Alstrum-Acevedo, J.; Bezel, I.; Meyer, T. J.; Klimov, V. I.; J. Am.Chem. Soc. 2006, 128, 9984.

47. Medintz, I. L.; Farrell, D.; Susumu, K.; Trammell, S. A.; Deschamps, J. R.; Brunel, F. M.; Dawson, P. E.; Mattoussi, H., Anal. Chem. 2009, 81, 4831.

48. Jorge, P. A. S.; Maule, C.; Silva, A. J.; Benrashid, R.; Santos, J. L.; Farahi, F., Anal. Chim. Acta 2008, 606, 223.

49. Guo, W.; Yuan, J.; Li, B.; Du, Y.; Ying, E.; Wang, E., Analyst 2008, 133, 1209.

50. Jorge, P. A. S.; Mayeh, M.; Benrashid, R.; Caldas, P.; Santos, J. L.; Farahi, F., Appl. Opt. 2006, 45, 3760.

51. McLaurin, E. J.; Greytak, A. B.; Bawendi, M. G.; Nocera, D. G., J. Am. Chem. Soc. 2009, 131, 12994. 
52. Anikeeva, P. O.; Madigan, C. F.; Coe-Sullivan, S. A.; Steckel, J. S.; Bawendi, M. G.; Bulovic, V., Chem. Phys. Lett. 2006, 424, 120.

53. Willard; D. M.; Carillo; L. L.; Jung;, J.; Orden., A. V., Nano Lett. 2001, 1, 469.

54. Wargnier;, R.; Baranov; A. V.; Maslov; V. G.; Stsiapura;, V.; Artemyev;, M.; Pluot;, M.; Sukhanova;, A.; Nabiev., I., Nano Lett. 2004, 4, 451.

55. Huang;, X.; Li; L.; Qian; H.; Dong:, C.; Ren, J., Angew. Chem. Int. Ed. 2006, 45, 5140

56. Plummer; E. A.; Hofstraat;, J. W.; Cola., L. D., Dalton Trans. 2003, 2080.

57. Sprouse, S.; King, K. A.; Spellane, P. J.; J., W. R., J. Am. Chem. Soc. 1984, 106, 6647.

58. Garces, F. O.; King, K. A.; Watts, R. J., Inorg. Chem. 1988, 27, 3464.

59. Ichimura, K.; Kobayashi, T.; King, K. A.; Watts, R. J., J. Phys. Chem. 2002, 91, 6104.

60. Lafolet, F.; Welter, S.; Popovic, Z.; Cola, L. D., J. Mater. Chem. 2005, 15, 2820.

61. Lo, K. K.-W.; Chung, C.-K.; Lee, T. K.-M.; Lui, L.-H.; Tsang, K. H.-K.; Zhu, N., Inorg. Chem. 2003, 42, 6886.

62. Benniston, A., C.; Harriman, A.; Patel, P., V.; Sams, C., A., Eur. J. Org. Chem. 2005, 2005, 4680.

63. Ohsawa, Y.; Sprouse, S.; King, K. A.; DeArmond, M. K.; Hanck, K. W.; Watts, R. J., J. Phys. Chem. 1987 91, 1047.

64. Wuister, S. F.; Driel, F. v.; Meijerink, A., Phys. Chem. Chem. Phys. 2003, 5, 1253.

65. Yu, W. W.; Qu, L.; Guo, W.; Peng, X., Chem. Mater. 2003, 15, 2854.

66. Meijerink, A., In Semiconductor nanocrystal quantum dots, Springer Vienna, 2008; pp 277.

67. Togano, T.; Nagao, N.; Tsuchida, M.; Kumakura, H.; Hisamatsu, K.; Howell, F. S.; Mukaida, M., Inorganica Chimica Acta 1992, 195, 221.

68. Broomhead, J. A.; Young, C. G., Inorg. Synth. 1982, 21, 127. 
69. Lay, P. A.; Sargeson, A. M.; Tauhe, H., Inorg. Synth. 1984, 24, 291.

70. Buckingham, D. A.; Dwyer, F. P.; Sargeson, A. M., Inorg. Chem. 1986, 5, 1243.

71. Dwyer, F. P.; Goodwin, H. A.; Gyarfas, E. C., Aust. J. Chem. 1963, 16, 42.

72. Buckingman, D. A.; Dwyer, F. P.; Sargeson, A. M., Aust. J. Chem. 1964, 17, 622 .

73. Miyaura, N.; Yamada, K.; Suzuki, A., Tetrahedron Letters 1979, 20 , 3437.

74. Welter, S.; Salluce, N.; Belser, P.; Groeneveld, M.; De Cola, L., Coord. Chem. Rev. 2005, 249, 1360.

75. Jurius, A.; Balzani, V.; Barigelletti, F.; Campagna, S.; Belser, P.; von Zelewsky, A., Coord. Chem. Rev. 1988, 84, 85.

76. Hughes, H. P.; Martin, D.; Bell, S.; McGarvey, J. J.; Vos, J. G., Inorg. Chem. 1993, 32, 4402.

77. Dubois, F.; Mahler, B.; Dubertret, B.; Doris, E.; Mioskowski, C., J. Am. Chem. Soc. 2006, 129, 482.

78. Meijerink, A., Exciton dynamics and energy transfer processes in semiconductor nanocrystals. 2008; pp 277.

79. Dexter, D. L., J. Chem. Phys. 1953, 21, 836.

80. Förster, T., Ann. Physik 1948, 2, 55.

81. Förster, T., In Fluorezenz. Organischer Verbindungen, Vandenhoech \& Ruprech: Göttingen, 1951.

82. Förster, T., Disc. Faraday Soc. 1959, 27, 7.

83. Lackowicz, J. R., In Principles of Fluorescence Spectroscopy, 3rd ed.; Springer 2006; pp 281. 


\title{
CHAPTER 4
}

\section{LUMINESCENT RUTHENIUM TRIPOD COMPLEXES: PROPERTIES IN SOLUTION AND ON CONDUCTIVE SURFACES}

\begin{abstract}
This chapter describes two luminescent ruthenium complexes containing tripod type terminal groups linked through a rigid spacer to a phenanthroline derivative, and being able to confer an axial geometry to the complexes, are described. One of the compounds is functionalized with acetylthiol groups in order to link the metal complex to metallic surfaces. The photophysical and electrochemical behavior of the complexes are studied in solution and on conductive substrates and furthermore self-assembled monolayer are investigated in a junction using $\mathrm{Au}$ and Eutectic Gallium Indium (EGAIN), as electrodes, and using time resolved confocal microscopy. The results show that the complexes form very stable and well ordered monolayers due to the tripod system that can anchor the complex almost perpendicular to the surfaces.
\end{abstract}




\section{Introduction}

In the last decades, a large number of papers dealing with various types of molecular assemblies on metallic and semiconductor surfaces have been published, since anchoring molecules on surfaces is the first step towards the creation of molecular devices. ${ }^{1-3}$ In particular, stable electro- and photoactive species possessing thiol derivative have been investigated to understand the role played by the chemical structures in important phenomena such as charge transport and conductivity in the general context of molecular electronics. ${ }^{4-11}$ Even though most of the investigated systems are organic molecules, more recently a lot of effort has been focused in the use of metal complexes containing $\mathrm{Ru}(\mathrm{II}), \operatorname{Ir}(\mathrm{III})$ and $\mathrm{Os}(\mathrm{II})$ ions coordinated to polypyridine derivatives and their assemblies on conductive surfaces. ${ }^{12-27}$ The advantages of using these molecules rely in their rich, often reversible, electrochemical properties which are often localized on the metal ion, for the oxidation processes, and on the ligands, for the reductions; in their photophysical properties, and in particular in the nature of their emitting excited state (triplet), and as a consequence long lived excited state lifetimes, good emission quantum yield and their stability. ${ }^{28}$ In addition, most of these systems have been lately fully investigated as materials for electroluminescent devices since their luminescent excited state can be electrically populated. ${ }^{29-30}$ The problem to anchor such metal complexes in well organized monolayers on conductive surfaces is mainly due to their octahedral geometry and therefore the arrangement of the chelating ligands. This problem is further compounded by the synthetic difficulties to introduce thiol groups. Also to control the distance and the assemblies' rigid ligands are preferable because they enable the metal complexes to stand perpendicular to the substrates. The most commonly employed ligands include amino, thiol and carboxylic groups as anchoring units. ${ }^{31-33}$ These groups have a tendency to bind to the surface of noble metals like gold, platinum and silver through both covalent and non-covalent interactions. However, from the application point of view the stability of these assemblies is of critical importance. Recently a few groups have reported the use of tripodal molecules in order to strongly bind the chromophores to the 
surface and to confer a better possibility for the molecule to stand on the substrate. ${ }^{34-39}$

In this chapter, following the same rationale as mentioned above, a rigid tripodal ruthenium complex with thioacetyl functionalities at each of the three legs has been employed. Furthermore, this chapter deals with the photophysical characterization of the tripodal complexes both in solution and their self-assembled monolayer (SAM) formation on conductive metallic substrates. In addition, the electrochemical behavior of these assemblies in solution and as SAMs is explored. This chapter concludes with the examination of the conductivity of the SAM between two electrodes measured using a Eutectic Indium Gallium (EGaIn) setup to demonstrate the possible application of these molecules in molecular electronics.

\section{Results and discussion}

All the ligands and $\mathrm{Ru}(\mathrm{II})$ complexes, as well as their abbreviations, are shown in Scheme 1. The synthesis of all these complexes was carried out by Dr. Fabio Edaffe from Prof. Peter Belser's group, University of Fribourg. 


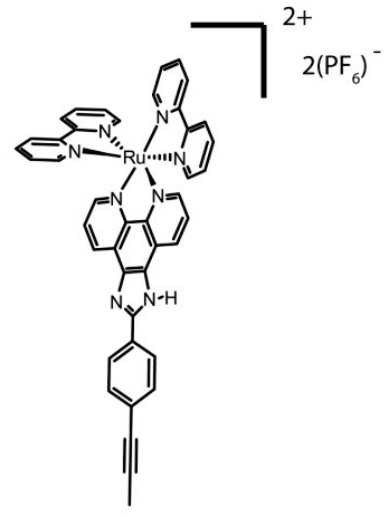

$[\text { Ru-(bpy) })_{2}$-EPEP] $\left(\mathrm{PF}_{6}\right)_{2}$

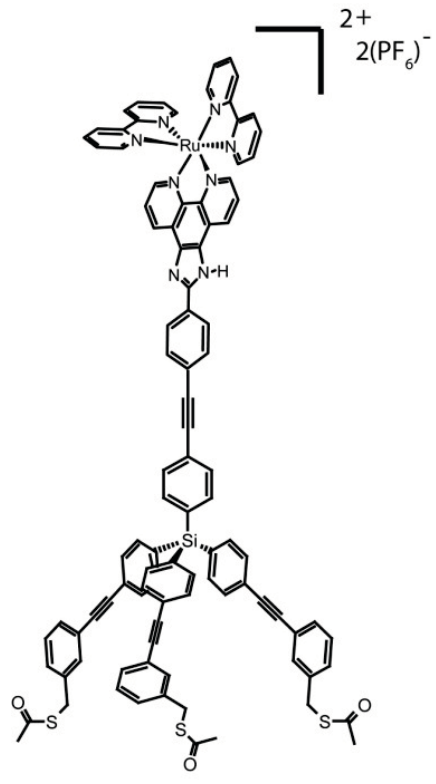

Ru-SAc

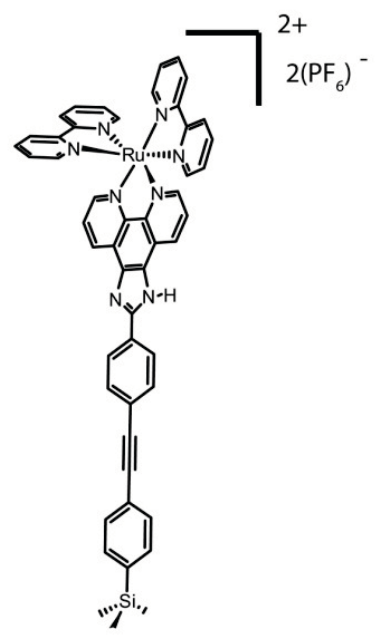

$[\text { Ru-(bpy) })_{2}$-TMS-EPIP] $\left(\mathrm{PF}_{6}\right)_{2}$

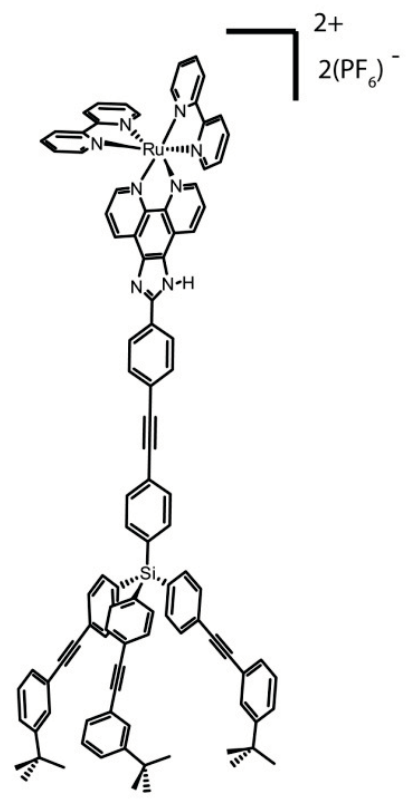

Ru-tert-Bu

Scheme 4.1. Schematic formulas of the complexes investigated and their precursors. The molecules will be named with the abbreviation shown under each formula. 


\section{Photophysical characterization in solution}

The absorption spectrum of Ru-SAc in acetonitrile solution is shown in Figure 4.1. The high energy bands at $291 \mathrm{~nm}\left(\varepsilon=1.08 \times 10^{5} \mathrm{~L} \cdot \mathrm{mol}^{-1} \cdot \mathrm{cm}^{-1}\right)$ can be assigned to the bipyridine and phenanthroline singlet intraligand ( $\left.{ }^{1} \mathrm{IL}\right) \pi \rightarrow \pi^{*}$ transitions, while the $312 \mathrm{~nm}\left(\varepsilon=8.1 \times 10^{4} \mathrm{~L} \cdot \mathrm{mol}^{-1} \cdot \mathrm{cm}^{-1}\right)$ and $341 \mathrm{~nm}(\varepsilon=3.7 \mathrm{x}$ $\left.10^{4} \mathrm{~L} \cdot \mathrm{mol}^{-1} \cdot \mathrm{cm}^{-1}\right)$ are due to the $\pi \rightarrow \pi^{*}$ absorptions of the highly conjugated moieties containing the phenylene ethylene groups. In particular, the lowest energy band is due to the (2-(4-(2-ethynyl)phenyl)-1H-imidazo[4,5-f][1,10]phenanthroline $)^{40}$ while the $312 \mathrm{~nm}$ feature is attributed to the tripod species. This assignment of the $312 \mathrm{~nm}$ feature is corroborated by the observation that for the two reference non-tripodal complexes, this $312 \mathrm{~nm}$ band is missing. The lowest energy bands around $458 \mathrm{~nm}\left(\varepsilon=1.5 \times 10^{4} \mathrm{~L} \cdot \mathrm{mol}^{-1} \cdot \mathrm{cm}^{-1}\right)$ are assigned to the singlet metal to ligand charge transfer $\left({ }^{1} \mathrm{MLCT}\right)$ transitions, which are typical for ruthenium polypyridyl complexes, involving the $\mathrm{d}$ orbitals of the ruthenium and the $\pi^{*}$ orbitals of the bipyridines and the phenantroline ligand.

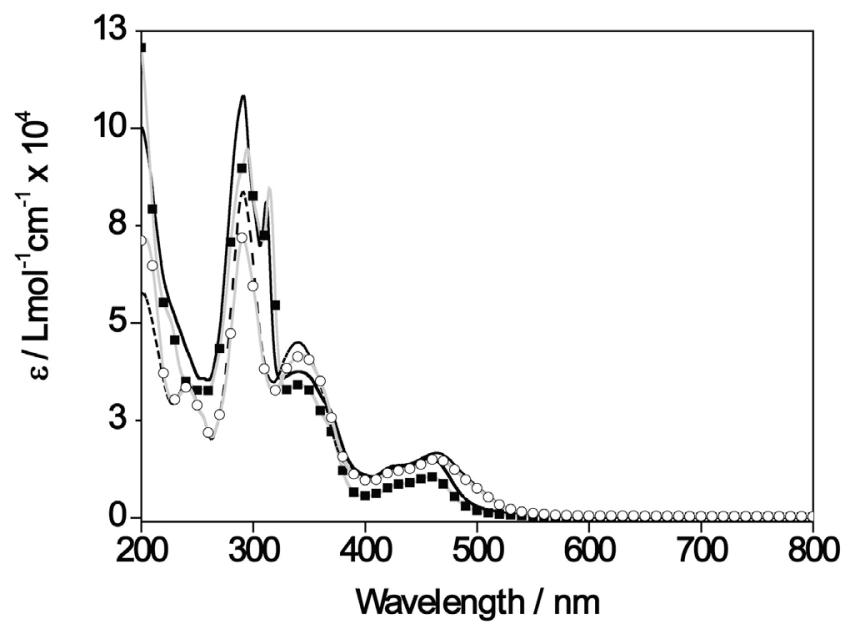

Figure 4.1: Absorption spectra of Ru-SAc (-), Ru-tert-Bu (-m-), Ru(bpy)2-TMS-EPIP $\left({ }^{\circ-}\right)$ and $\mathrm{Ru}(\mathrm{bpy})_{2} \mathrm{EPIP}(---)$ complexes in acetonitrile solutions. 
Due to the similar energy levels involving the bipyridine and the phenanthroline and the broadness of the spectra, it is difficult to attribute the lowest excited state from the absorption characteristics. However, one can expect that for the complexes containing the tripod with the thioacetate and the tertiarybutyl groups the lowest MLCT involves the bipyridine ligands. On the other hand the Ru(bpy) $)_{2}$ EPIP and Ru(bpy) $)_{2}$ TMS-EPIP, due to the higher conjugation and lack of electron donating groups, should have the lowest MLCT absorption on the phenanthroline moiety. Such hypothesis is supported by the emission spectra and electrochemistry data of the complexes
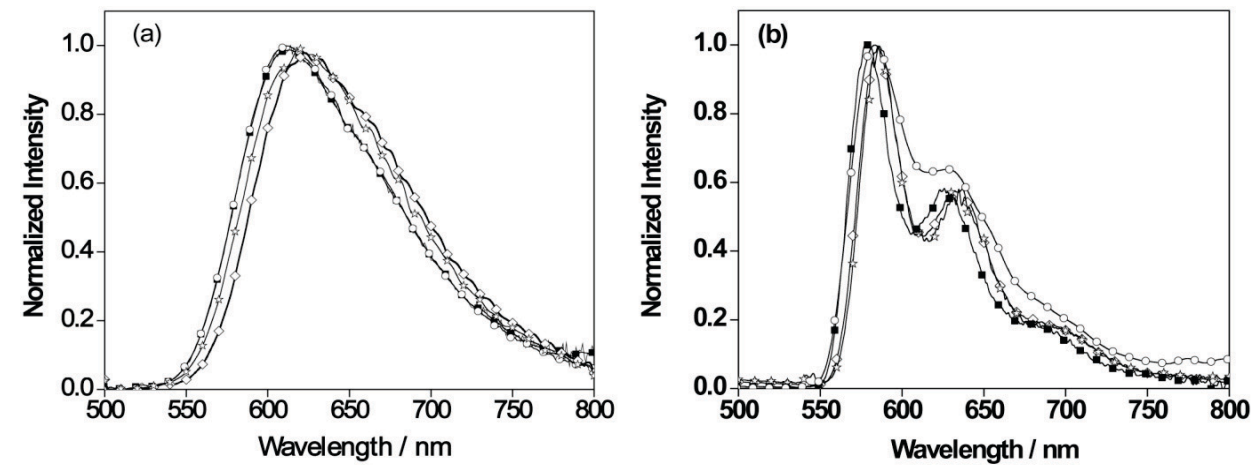

Figure 4.2: (left) Emission spectra of Ru-SAc (-), Ru-tert-Bu (-m-), Ru(bpy)2TMSEPIP(-o-) and Ru(bpy) ${ }_{2}$ EPIP (---) complexes in acetonitrile solutions measured at room temperature. (right) Emission spectra of of Ru-SAc (-), Ru-tert-Bu (- -), $\mathrm{Ru}(\mathrm{bpy})_{2}$ TMS-EPIP (-O-) and Ru(bpy) ${ }_{2} \mathrm{EPIP}$ (---) complexes in acetonitrile solutions measured at $77 \mathrm{~K}$ in butyronitrile glass. $\lambda_{\mathrm{ex}}=452 \mathrm{~nm}$.

The room temperature emission spectra of the reference compound Ru-tbu and $\mathrm{Ru}-\mathrm{SAc}$ complexes were recorded in acetonitrile solution at $452 \mathrm{~nm}$ excitation (Figure 4.2). The emissions have broad structureless bands centered at $609 \mathrm{~nm}$ and $614 \mathrm{~nm}$ for Ru-SAc and Ru-tbu respectively. These bands are attributed to the radiative decay of triplet metal to ligand charge transfer $\left({ }^{3} \mathrm{MLCT}\right)$ state of the ruthenium complexes to the ground state. The red shift of the emission band for the Ru-tert-Bu complex vs. the Ru-SAc can be attributed to the slightly electron-donating nature of the tertiary butyl groups and therefore it is possible to assign as the lowest excited state the ${ }^{3}$ MLCT to the bpy ligands. The excited state lifetimes for both the complexes are very similar in aerated as well as in deaerated acetonitrile solutions (see Table 4.1). 
Deaerated solutions show much longer excited state lifetimes as expected due to the triplet character of the lowest excited states. The emission quantum yields of the complexes were measured using $\mathrm{Ru}(\mathrm{bpy})_{3}{ }^{2+}$ complex as the reference $\left(\Phi_{\mathrm{em}}=0.016\right)^{41}$ in aerated acetonitrile and the values are reported in Table 4.1. In the deaerated solutions, as expected, the emission quantum yields of the complexes are higher than for the analogous aerated complexes. The precursor complexes also emit at room temperature and their emission is quite similar to the other compounds. In the same solvent they show emission maxima that are slightly red shifted compared with the tripod systems, $\lambda_{\text {em }}=$ $617 \mathrm{~nm}$, and, $\lambda_{\text {em }}=621 \mathrm{~nm}$ for $\mathrm{Ru}(\mathrm{bpy})_{2}$ EPIP and Ru(bpy) $)_{2}$ TMS-EPIP, respectively. The $77 \mathrm{~K}$ emission spectra, recorded in butyronitrile glass, (Figure 4.2) show a blue shift relative to the RT solution measurements as expected for an MLCT emission similar to what is known for Ru-polypyridyl complexes. ${ }^{42}$ The excited state lifetimes at $77 \mathrm{~K}$ (Table 4.1) are longer than the RT. Such a behavior is attributed to the lack of thermal population of the triplet metal centered $\left({ }^{3} \mathrm{MC}\right)$ states that would quench the excited state emission at higher temperature. $^{43}$ 


\begin{tabular}{|c|c|c|c|c|c|c|c|}
\hline \multirow[t]{2}{*}{ Complex } & \multicolumn{5}{|c|}{ Emission, RT } & \multicolumn{2}{|c|}{ Emission, $77 \mathrm{~K}$} \\
\hline & $\begin{array}{l}\lambda_{\max } \\
(\mathrm{nm})\end{array}$ & $\phi^{\mathrm{a}}$ & $\phi^{\mathrm{b}}$ & $\begin{array}{c}\tau^{\mathrm{a}} \\
(\mu \mathrm{s})\end{array}$ & $\begin{array}{c}\tau^{\mathrm{b}} \\
(\mathrm{ns})\end{array}$ & $\begin{array}{l}\lambda_{\max }{ }^{\mathrm{c}} \\
(\mathrm{nm})\end{array}$ & $\begin{array}{c}\tau^{\mathrm{c}} \\
(\mu \mathrm{s})\end{array}$ \\
\hline $\mathrm{Ru}-\mathrm{SAc} \mathrm{c}^{\mathrm{d}}$ & 609 & 0.12 & 0.014 & 1.04 & 155 & $\begin{array}{l}579 \\
626\end{array}$ & 5.2 \\
\hline Ru-tert-Bu ${ }^{d}$ & 614 & 0.14 & 0.018 & 1.14 & 196 & $\begin{array}{l}580 \\
628\end{array}$ & 5.6 \\
\hline $\mathrm{Ru}(\mathrm{bPy})_{2} \mathrm{EPIP}$ & 617 & 0.08 & 0.015 & 1.08 & 178 & $\begin{array}{l}586 \\
635\end{array}$ & 5.3 \\
\hline $\begin{array}{c}\mathrm{Ru}(\mathrm{bpy})_{2} \mathrm{TMS}- \\
\text { EPIP }\end{array}$ & 621 & 0.10 & 0.020 & 1.05 & 203 & $\begin{array}{l}585 \\
637\end{array}$ & 5.2 \\
\hline Ru-SH - gold & 619 & - & - & 2.0 & 8.0 & - & - \\
\hline $\begin{array}{l}\text { Ru-SAc on } \\
\text { glass }\end{array}$ & 615 & - & - & 90 & 812 & & \\
\hline
\end{tabular}

${ }^{a}$ In degassed solution. ${ }^{b}$ In air-equilibrated solution ${ }^{c}$ In butyronitrile glass. For lifetime measurements $402 \mathrm{~nm}$ laser diode was used as excitation source. For lifetime on gold and on glass, $440 \mathrm{~nm}$ laser excitation was used.

Table 4.1: Photophysical data in acetonitrile solution, otherwise specified, of the RuSAc and reference and precursor complexes.

\section{Preparation of Self Assembled Monolayers (SAMs)}

In order to perform local measurements on the SAMs a surface with a very high degree of flatness is required. Hence, we prepared the ultraflat gold substrates on microscope cover slip using the template stripping (TS) method as described by Weiss and coworkers. ${ }^{44}$ Briefly, first the gold was evaporated on Si wafers and then glued on the cleaned (sonicated in EtOH and Plasma activated) microscope cover slips by using Optical Adhesive (Norland 61). The material was then cured for $2 \mathrm{~h}$ under UV light (200 W Hg lamp with $280-400$ $\mathrm{nm}$ dichroic filter). Following this procedure, the edges of the glass were carved and the glass substrate was lifted off with the edge of a scalpel. The thin flat Au layer was therefore immobilized on the microscope glass. The samples were immediately immersed in an $\mathrm{EtOH}$ solution of the tripod molecules and left for $24 \mathrm{~h}$ for monolayer formation. $10 \mu \mathrm{L}$ of hydrazine solution was added 
to deprotect the thioacetyl groups in-situ to facilitate the Ru-SH monolayer formation. The samples were subsequently rinsed with EtOH to remove any unbound molecules. As the AFM picture shows (Figure 3) the monolayer is not homogeneous but forms islands which is well known for other thiolate complexes on surfaces ${ }^{1,26}$. The same procedure was applied to the reference compound Ru-tert-Bu. Monolayer was not observed with Ru-tert-Bu due to the lack of anchoring groups, which does not allow any strong bond to the $\mathrm{Au}$ surface and therefore after the first wash the complex was rinsed away. The AFM picture indicates islands of Ru-SAc are sufficiently large to perform local measurements and the quality and height of the layers confirm the monolayer formations and their stability. Their good packing was also indicated by the high yields in the EGaIn measurements (vide infra). When a bare gold surface was immersed in the ethanolic solution under identical conditions no such islands formed supporting our claim that the islands are indeed due to the monolayer formation. This experiment also suggested that the depth profile of about $3 \mathrm{~nm}$ that is indicated by the AFM is not due to the surface roughness of the gold. 

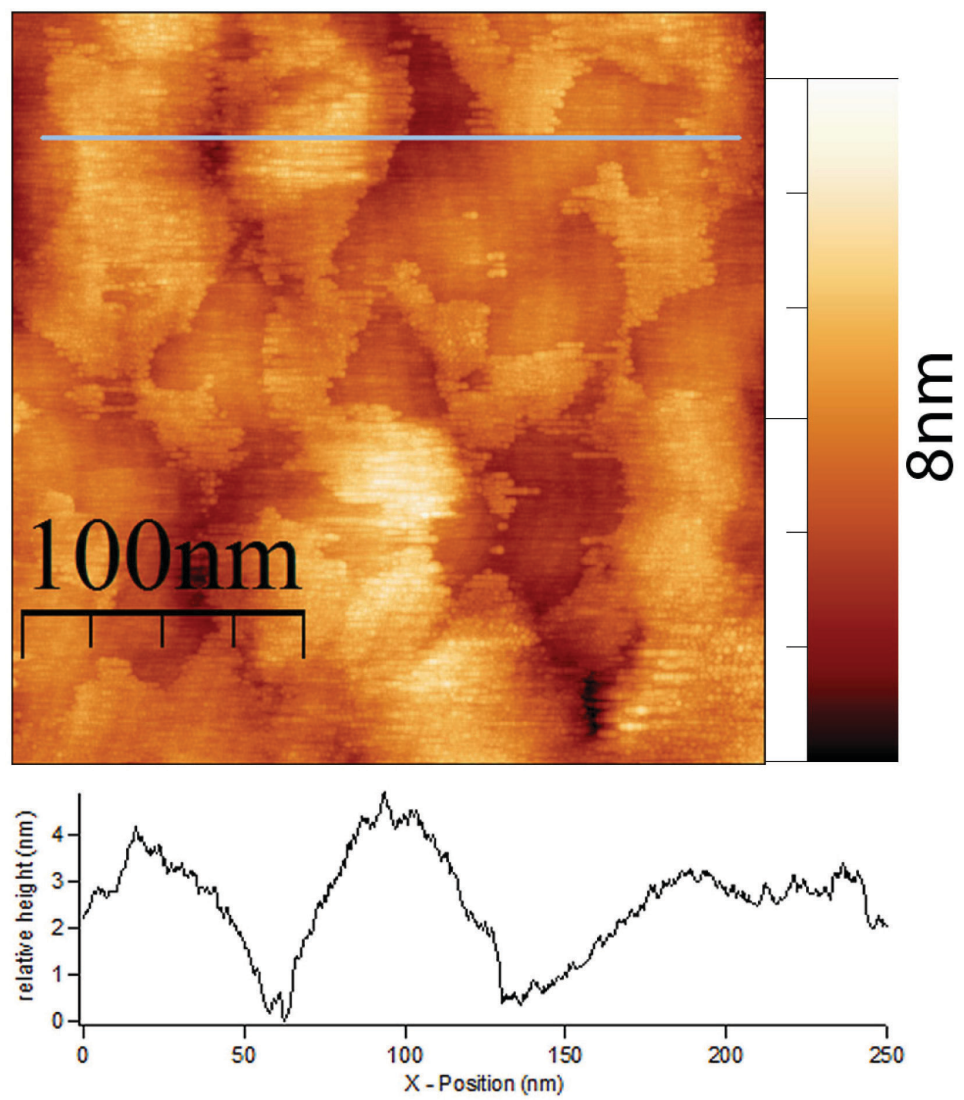

Figure 4.3: AFM picture of self-assembled monolayer of Ru-SH on Au substrate and height profile, revealing the formation of islands with their heights correspond to the dimensions of the molecule.

\section{Photophysical measurements of Ru-SH monolayer}

The emission measurements of the Ru-SAc monolayer on ultra-flat gold substrate were successfully carried out in the spectrofluorimeter indicating that despite the quenching induced by the Au surface the signal strength from the monolayer was detectable. The emission of monolayer (Figure. 4.4) resembled the emission profile of Ru-SAc in solution. However, we see a red shift of 10 $\mathrm{nm}$ in the emission with respect to the solution measurements. To compare the effect of the gold surface on the emission of the monolayer, a solution of Ru-SAc was drop cast on a glass cover slip and its emission was measured in a 
spectrofluorimeter. Ru-SAc monolayer emission was red shifted by $4 \mathrm{~nm}$ compared to that on the glass. This is indicative of the change in the excited state properties upon strong electronic interaction with the gold surface. In other words, upon anchoring the complexes on the substrate the ${ }^{3} \mathrm{MLCT}$ would be localized on the chelating (2-(4-(2-ethynyl)phenyl)-1H-imidazo[4,5f] $[1,10]$-phenanthroline) moiety. The excitation spectrum of the monolayer is shown in Figure 4.4.
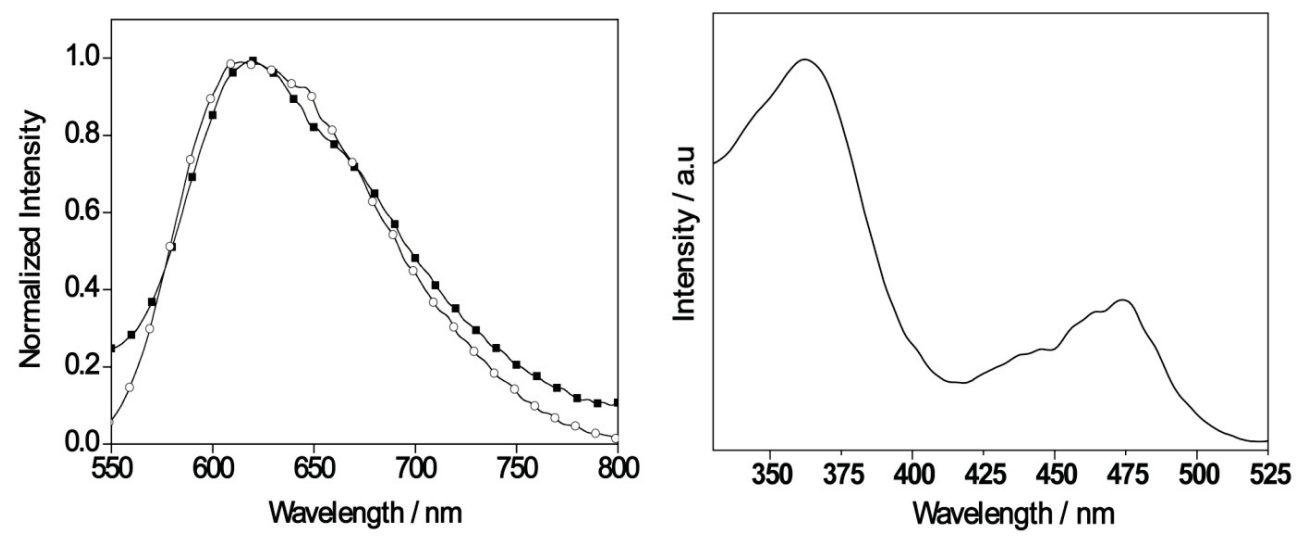

Figure 4.4: (left) Emission spectra of Ru-SH monolayer on ultraflat gold substrate (--) and Ru-SAc drop cast on glass cover slip (- $\left.{ }^{\circ}\right)$. (right) Excitation spectrum of Ru-SAc monolayer on gold substrate measured by monitoring the emission at $610 \mathrm{~nm}$. 


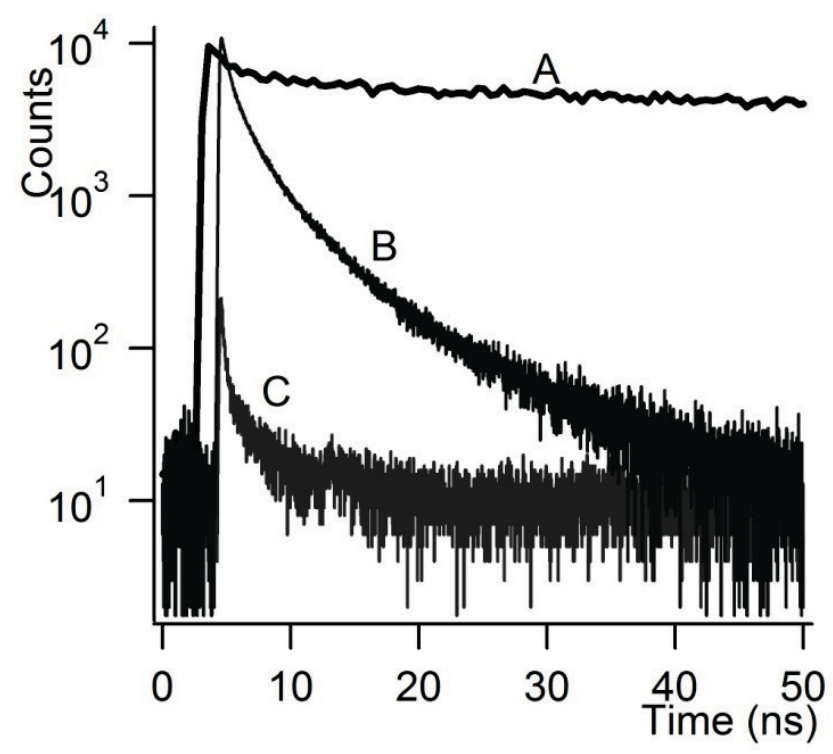

Figure 4.5: Decay profiles of (A) Ru-SAc on glass; (B) Ru-SH on gold; (C) Bare gold. $\lambda_{\text {ex }}=440 \mathrm{~nm}$.

The excited state lifetime of the immobilized Ru-SAc molecules were measured using a time resolved confocal microscope. The lifetimes were fitted to a biexponential with a shorter component of $2 \mathrm{~ns}$ and a longer component of $8 \mathrm{~ns}$ (Figure 4.5). This dual exponential decay could be due to the different orientation of the molecules on the surfaces; for instance the coordination of two out of the three thiols or the perpendicular orientation of the Ru-complex upon coordination of all the three anchoring groups. The XPS measurements (Figure 4.6) show a doublet for $\mathrm{S}_{2 \mathrm{p}}$ peak for sulfur (at 164 and $169 \mathrm{eV}$ respectively). The former value could be attributed to the sulfur moiety that is bound to the gold surface while the latter one is due to the unbound sulfur. Further, the ratio in intensity between these two peaks is 2:1 indicating that two out of the three groups surely are attached to the Au surface. This result is consistent with what was reported for similar systems. ${ }^{45}$ Another possible explanation for the presence of two components is triplet-triplet annihilation due to strong packing of the complexes with a consequent quenching of the emission. In order to evaluate the extent of the quenching, a film of Ru-SAc was prepared by drop cast method on a microscopy glass plate and 
subsequently the lifetime was measured. The lifetime was fit to a bi-exponential decay. The long component has an excited state lifetime of 812 ns and the shorter component of $90 \mathrm{~ns}$. This is in good agreement with the value reported for other ruthenium complexes on glass $(700 \pm 50 \mathrm{~ns})^{46}$. Since the glass does not exert any electronic interaction with the ruthenium complex, the short component could possibly be due to triplet-triplet annihilation. The long lifetime can be therefore taken as a good reference for the unquenched ruthenium complex on a substrate.

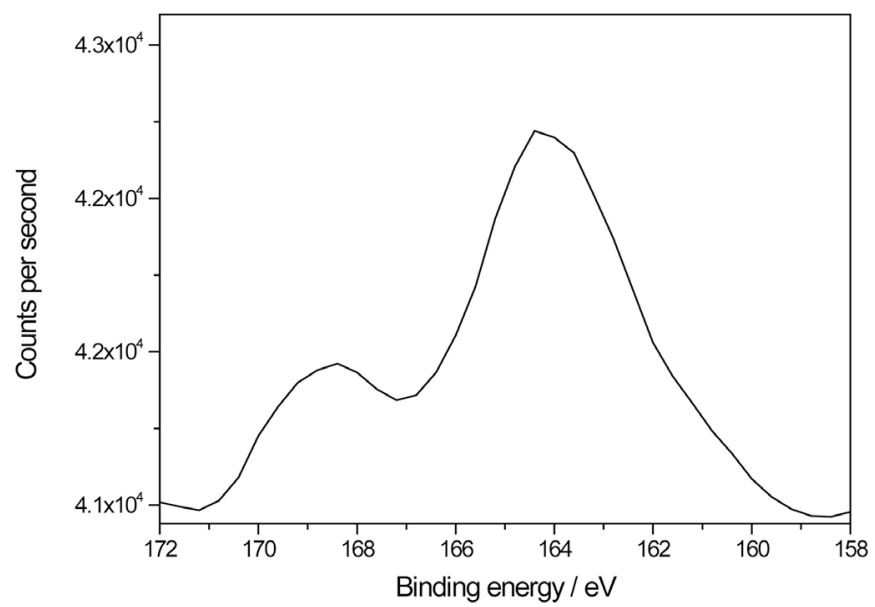

Figure 4.6: XPS spectrum of Ru-SH bound to gold substrate indicating two binding energies for sulfur

In the case of the SAM it is reasonable to conclude that the quenching of the emission is due to a photoinduced energy and/or electron transfer from the metal complex to the gold surface as reported earlier. ${ }^{47}$ In particular for similar ruthenium complexes anchored onto the gold nanocrystalline surface, it is reported that the origin of quenching comes mainly from an electron transfer. ${ }^{48}$ The quenching rate can be calculated using the following equation using the 8 ns lifetime for Ru-SAc on Au and 812 ns for Ru-SAc on glass.

$$
\mathrm{k}_{\mathrm{eT}}=1 / \tau-1 / \tau_{0}
$$

Using this equation, the rate constant was determined to be $1.23 \times 10^{8} \mathrm{~s}^{-1}$. This estimated rate is about three orders of magnitude faster than that reported by 
Unwin and coworkers for thiolated ruthenium complex on gold surface $(1 \mathrm{x}$ $\left.10^{-5}\right)^{49}$ and about two orders of magnitude faster than that reported by Kamat and co-workers for electron transfer from a ruthenium polypyridyl complex to gold nanoparticles $\left(1.1 \times 10^{6} \mathrm{~s}^{-1}\right)^{48}$. However, in both the above cases, the ruthenium complexes employed have alkyl linkers to the gold surface compared to the conjugated tripodal leg in our system, which can facilitate faster electron transfer.

\section{Electrochemical measurements}

Electrochemistry of the reference compound, Ru-tert-Bu, as well as the thiolated Ru-Sac, was performed in solution. The Ru-SAc electrochemistry was compared with the results obtained on the self-assembled monolayer (SAMs) on a Pt-wire electrode. In acetonitrile solution with tetrabutyl ammonium hexafluorophosphate $\left(\mathrm{TBAPF}_{6}\right)$ as electrolyte, both the complexes show reversible reduction and oxidation waves. In particular the first oxidation occurs at $+0.91 \mathrm{~V}$ (vs. Ferrocene). The peak to peak separation is $90 \mathrm{mV}$. The reversible oxidation can be attributed to the oxidation of the $\mathrm{Ru}$ center $\mathrm{Ru}^{\mathrm{III}} / \mathrm{Ru}^{\mathrm{II}}$ and occurs at potentials similar to the $\left[\mathrm{Ru}(\mathrm{bpy})_{2} \text { (phen) }\right]^{2+} .^{50}$ The first reduction at $-1.73 \mathrm{~V}$ can be attributed to the reduction of the bipyridine ligand. 


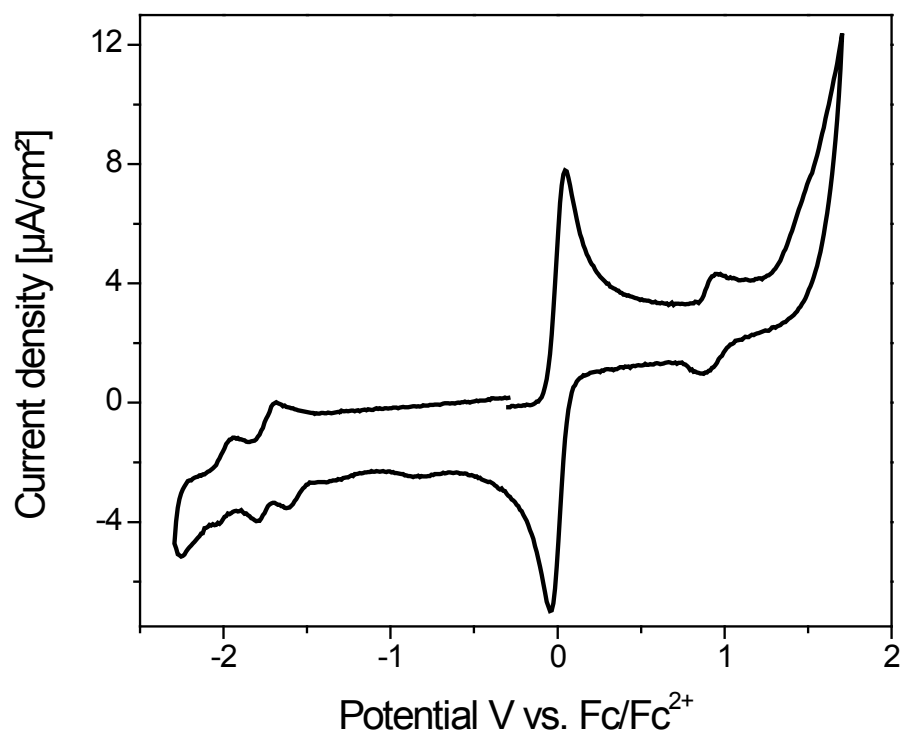

Figure 4.7: Electrochemistry of Ru-tert-Bu in neat acetonitrile solution with $0.1 \mathrm{M}$ $\mathrm{TBAF}_{6}$ as the electrolyte. Scan rate $500 \mathrm{mv} / \mathrm{s}$

For electrochemistry on monolayers a Pt electrode was pre-cleaned thoroughly by repeated sonication with deionized water and acetonitrile (spectroscopic grade). Ru-SAc monolayers were formed by immersion of this pre cleaned $\mathrm{Pt}$ electrode in a concentrated solution of Ru-SAc in acetonitrile $\left(\sim 10^{-3} \mathrm{M}\right)$. The acetyl thiol groups were deprotected in-situ by adding $\sim 10 \mu \mathrm{l}$ of hydrazine solution. The electrode was later rinsed with acetonitrile solution before measurements. The cyclic voltammogramms were measured with different scan rates and referenced against ferrocene. The oxidation of the Ruthenium ion occurs at $+0.98 \mathrm{~V}$, very similar to the value obtained in solution for the reference Ru-tert-Bu complex. It is interesting to note that the peak separation $(\Delta \mathrm{Ep})$ is greater than 0 and increases with increasing scan rates. $(210 \mathrm{mV}$ at $1000 \mathrm{mV} / \mathrm{s}$ as compared to $52 \mathrm{mV}$ at $100 \mathrm{mV} / \mathrm{s}$, see Figure 4.8). This is consistent with similar trends observed for Os bipyridine complexes on $\mathrm{Au}$ surfaces. ${ }^{51}$ In addition to this, the full width at half maximum (FWHM) is greater than $90.6 \mathrm{mV}$, which is the expected value for an ideal one-electron redox process. Both these observations can be attributed to repulsive 
interactions between neighboring redox sites that becomes significant at reasonably higher packing density of the monolayer ${ }^{52-54}$ as indicated in this case by AFM and monolayer measurements (see next section).

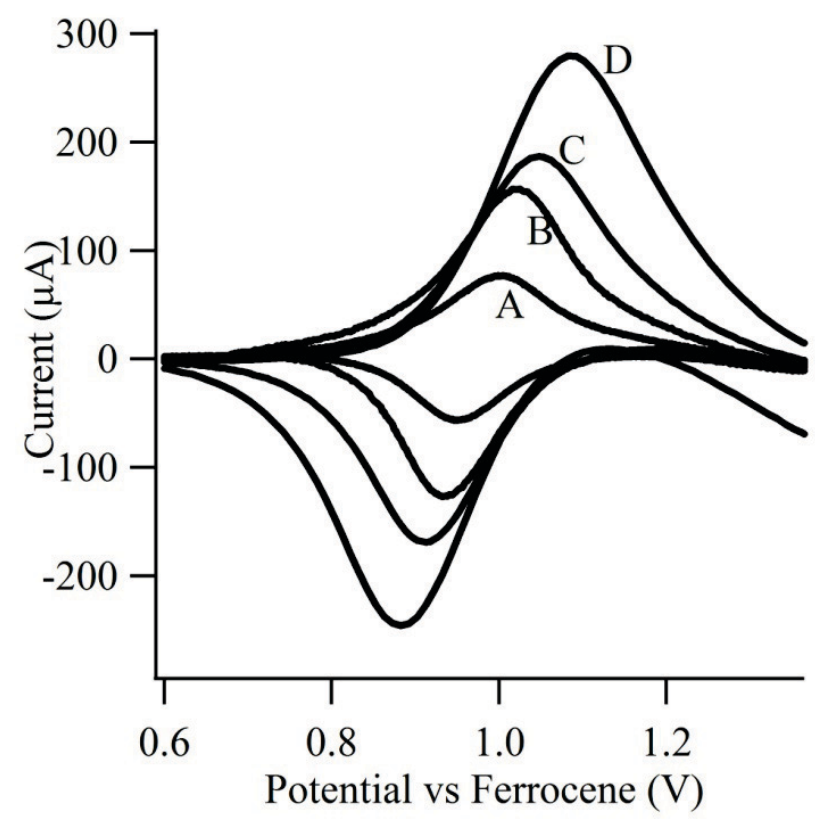

Figure 4.7: $\mathrm{CV}$ of Ru-SH adsorbed onto the platinum electrode recorded in acetonitrile solution at different scan rates. (A) $100 \mathrm{mV} / \mathrm{s}$ (B) $200 \mathrm{mV} / \mathrm{s}$ (C) 500 $\mathrm{mV} / \mathrm{s}$ (D) $1 \mathrm{~V} / \mathrm{s}$

The surface coverage, $\Gamma$, can be estimated using electrochemical methods. This is done by calculating the area under the voltammetric peak by incorporating the correction for double layer charging in the equation

$$
\Gamma=\frac{Q}{n F A}
$$

where $\mathrm{Q}$ is the total charge required to oxidize or reduce the electroactive species, $\mathrm{n}$ is the number of electrons involved in the electrochemical process, $\mathrm{F}$ is the Faraday's constant which has a value $96485.3415 \mathrm{~s} \mathrm{~A} \mathrm{/} \mathrm{mol} \mathrm{and} \mathrm{A} \mathrm{is} \mathrm{the}$ area of the electrode. The first oxidation peak $(100 \mathrm{mv} / \mathrm{s}$ in Figure 4.7$)$ was 
used to calculate the coverage in the present system and the oxidation for ruthenium molecule is a one electron process. The area of the electrode used was $0.314 \mathrm{~cm}^{2}$. From the $\mathrm{CV}$, the area under the first oxidation peak was estimated to be $1.5 \times 10^{-7} \mathrm{C}$. Employing all these values in the above equation yielded a total surface coverage of $5.01 \times 10^{-12} \mathrm{~mol} / \mathrm{cm}^{2}$.

\section{Conductivity measurements using EGaIn setup}

In order to gain a better picture of how the electrons and holes can flow through the molecules, we formed tunneling junctions of SAMs of Ru-SAc. We used conically shaped EGaIn top-electrodes ${ }^{55-56}$ and template striped (TS) $\mathrm{Au}$ surfaces as bottom electrodes to construct tunnel junctions containing SAMs of Ru-SAc. ${ }^{44}$ A schematic picture of the tunneling junctions is shown in Figure 4.8. We used EGaIn top-electrodes because these junctions are easy to assemble and the EGaIn does not destroy the molecules, unlike, for instance, direct metal deposition methods. Early work has shown that a liquid electrode, such as a mercury drop, could be the answer to measure rather fragile monolayers ${ }^{57}$. However, the mechanical stability of the $\mathrm{Hg}$-based junction is not so high and indeed the use of this electrode is rather impractical for further developments. Junctions based on $\mathrm{Hg}$-drop top-electrodes only were stable, in the best cases, up to 15 scans before the $\mathrm{Hg}$ top-electrode amalgamates with the bottom-electrode. EGaIn as a top electrode overcomes some of these problems and proved to be a possible answer in terms stability and reproducibility. ${ }^{55}$ The EGaIn top-electrodes were biased while the TS Au electrodes were grounded. 


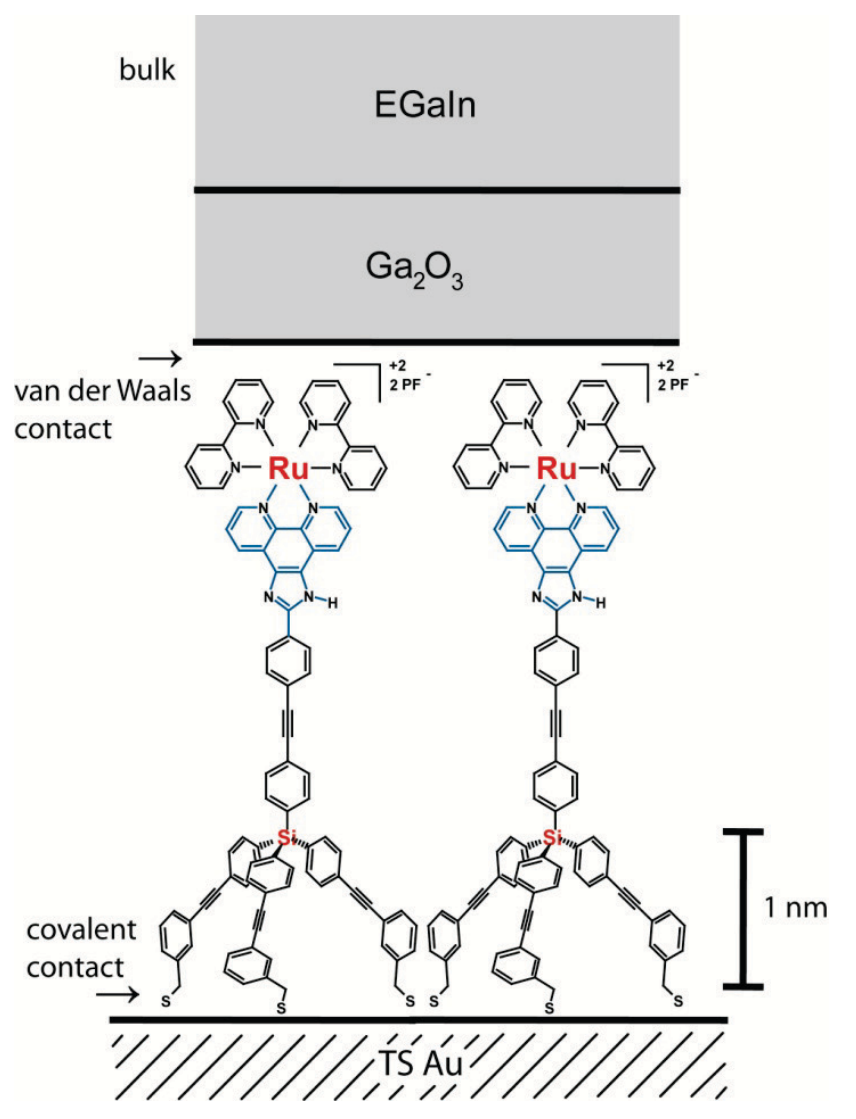

Figure 4.8: A schematic of the TS Au-Ru-tripod//EGaIn tunnel junctions. The

EGaIn top-electrode is biased and the TS Au bottom-electrode is grounded.

The Ru-SAc monolayers were formed at the TS Au electrodes for $24 \mathrm{~h}$. Contacting the monolayer with a conically shaped tip of EGaIn completed the junctions. Typical junction sizes were $500-1000 \mu \mathrm{m}^{2}$. A total of 17 junctions were assembled at three different TS Au surfaces. Of these 17 junctions, 14 were working and 3 junctions were shorting, thus the yield of working devices was $82 \%$. The junctions were then scanned between -2 and $+2 \mathrm{~V}$. An example of a $J(\mathrm{~V})$ curve and the corresponding semi-log plot of a $|J|(\mathrm{V})$-curve is shown in Figure 4.9. The junctions were stable against these large potentials and they could be measured for more than $1 \mathrm{~h}$ obtaining 20 - 30 scans before they shorted or the experiments were stopped. Of one junction we measured 60 
traces before we terminated the experiment. The relatively large stability and the high yield in working devices suggest that the tripod molecules are extremely stable and most likely constantly standing up and that the SAMs are densely packed.

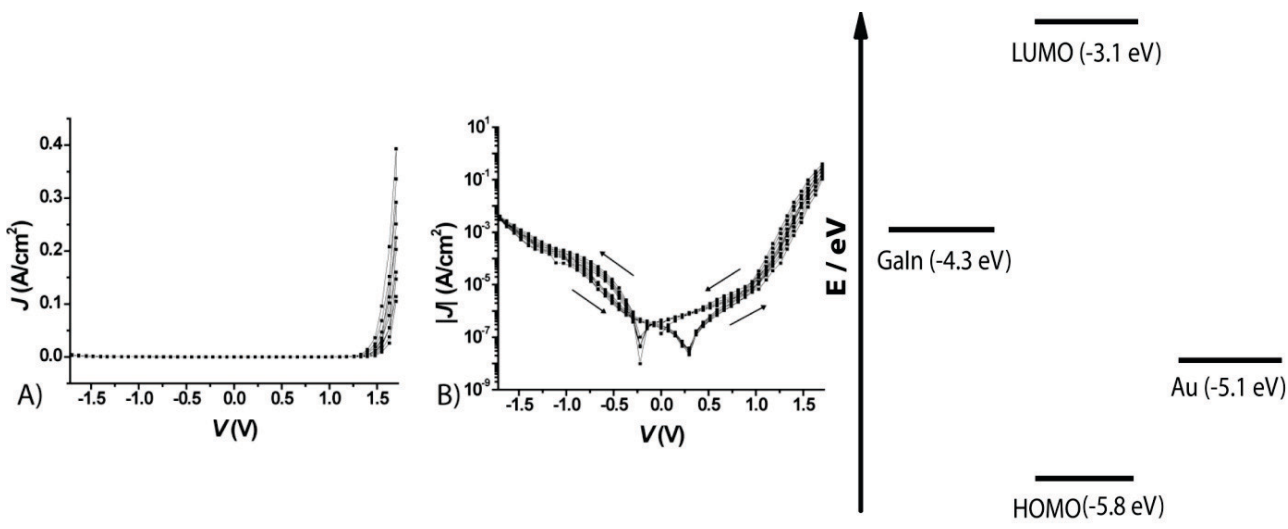

Figure 4.9: (left) Five J(V)-traces of a TS Au-Ru//EGaIn junctions (A) and the corresponding $|\mathrm{J}|(\mathrm{V})$ semi log-plot. One trace $=0 \mathrm{~V} \rightarrow+1.5 \mathrm{~V} \rightarrow-1.5 \mathrm{~V} \rightarrow 0 \mathrm{~V}$ and the arrows indicate the scan direction. (right) Energy levels of the Ru-SH molecule and the electrodes in EGaIn setup.

The TS Au-Ru//EGaIn junctions rectify currents with a rectification ratio $\mathrm{R}=$ $(|J|(\mathrm{V}) /|J|(-\mathrm{V})) \sim 100$ at $\pm 1.5 \mathrm{~V}$. The origin of the rectification in these junctions is not fully understood. The Ru-tripod has accessible HOMO and LUMO levels which may come in resonance with the Fermi-levels of the electrodes at large bias. The HOMO level is located at the Ru-center and the LUMO level is located at the phenanthroline ligand which is indicated as a blue ligand, Figure. 4.9. The tripod molecule studied here is a highly conjugated molecule, thus the HOMO and LUMO orbitals will, to a certain degree, be delocalized. Given the length of the molecule, it is fair to assume that both, the HOMO and LUMO orbitals will be located asymmetrically inside the junction. For this reason, the orbitals are in principle asymmetrically coupled to the electrodes and should be coupled more strongly to the EGaIn top-electrode. We believe that the HOMO and LUMO levels are asymmetrically coupled to the bottom- and top-electrodes, but the presence of mobile $\mathrm{PF}_{6}^{-}$anions in the 
junction will, at least partially, compensate for that. The presence of mobile $\mathrm{PF}_{6}^{-}$anions in the junctions may cause large potential drops at the tripod// $\mathrm{Au}$ interface facilitating the holes injection process.

The HOMO and LUMO level were estimated by electrochemistry data and found to be $-5.8 \mathrm{eV}$ and $-3.1 \mathrm{eV}$, respectively. The EGaIn has a work function of $\sim-4.3 \mathrm{eV}$ and $\mathrm{Au}$ of $-5.1 \mathrm{eV}$. The $|J|(\mathrm{V})$-curve shows two sudden increase in current density at $-1.3 \mathrm{~V}$ and $0.9 \mathrm{~V}$. This increase in current density may reflect the on- and off-resonance tunneling of the molecular HOMO and LUMO levels of the Ru-tripod. Thus at $>0.9 \mathrm{~V}$ bias, holes are injected in the HOMO level, while $>-1.3 \mathrm{~V}$ bias electrons are injected in the LUMO. Since these processes take place at different potentials, we observe rectification with larger currents at positive bias. More experiments with similar systems are warranted to completely understand the mechanism of charge transport in such systems.

\section{Conclusions}

In this chapter we have reported the photophysical investigations of a series of ruthenium complexes, two of them containing a tripod derivative. The tripod can be further chemically functionalized with thiol groups for its attachment to metallic surfaces. The geometry of the complexes has been designed so that the ruthenium center is coordinated to 2 bipyridine ligands and a chelating phenanthroline bearing a 5 member ring for its axial functionalization. This approach allows the complexes to be luminescent and, for the thiol derivative, to stand almost vertically when assembled to a surface. We have demonstrated that the Au surface quenches the emission of the ruthenium complexes selfassembled on the metal but allows the construction of redox active junction to investigate the conductivity of the assembled molecules. Using electrochemistry we have determined the surface coverage of the monolayer on a Pt electrode and is estimated to be $5.01 \times 10^{-12} \mathrm{~mol} / \mathrm{cm}^{2}$. We have investigated this junction using as a second electrode a EGaIn eutectic. The results showed that the monolayers are extremely stable and a rectification behavior is observed. In view of the fact that the complexes are also electroluminescent, the understanding of the charge injection and transport 
could lead to important consequences for the design of LEEC and other electroluminescent devices.

\section{Experimental Secition}

\section{Photophysics:}

Absorption spectra were measured on a Varian Cary 5000 double-beam UVVis-NIR spectrometer and baseline corrected. Steady-state emission spectra were recorded on a HORIBA Jobin-Yvon IBH FL-322 Fluorolog 3 spectrometer equipped with a $450 \mathrm{~W}$ xenon arc lamp, double grating excitation and emission monochromators $(2.1 \mathrm{~nm} / \mathrm{mm}$ dispersion; 1200 grooves $/ \mathrm{mm}$ ) and a Hamamatsu R928 photomultiplier tube or a TBX-4-X single-photoncounting detector. Emission spectra were corrected for source intensity (lamp and grating) and emission spectral response (detector and grating) by standard correction curves. For monolayers, the sample was mounted on a commercially available solid-state sample holder provided by HORIBA Jobin-Yvon, and the emission was collected with the front face geometry. Time-resolved measurements were performed using the time-correlated single-photon counting (TCSPC) option on the Fluorolog 3. NanoLED (402 nm; FWHM < $750 \mathrm{ps}$ ) with repetition rates between $10 \mathrm{kHz}$ and $1 \mathrm{MHz}$ were used to excite the sample.

Quantum yield: Luminescence quantum yields ( $\Phi$ em) were measured in optically dilute solutions (O.D. $<0.1$ at excitation wavelength) and compared to reference emitters by the following equation:

$$
\Phi_{x}=\Phi_{r}\left[\frac{A_{r}\left(\lambda_{r}\right)}{A_{x}\left(\lambda_{x}\right)}\right]\left[\frac{I_{r}\left(\lambda_{r}\right)}{I_{x}\left(\lambda_{x}\right)}\right]\left[\frac{n_{x}^{2}}{n_{r}^{2}}\right]\left[\frac{D_{x}}{D_{r}}\right]
$$

where $\mathrm{A}$ is the absorbance at the excitation wavelength $(\lambda), \mathrm{I}$ is the intensity of the excitation light at the excitation wavelength $(\lambda), \mathrm{n}$ is the refractive index of the solvent, D is the integrated intensity of the luminescence and $\Phi$ is the quantum yield. The subscripts $\mathrm{r}$ and $\mathrm{x}$ refer to the reference and the sample, respectively. All quantum yields were performed at identical excitation wavelength for the sample and the reference, canceling the $\mathrm{I}(\lambda \mathrm{r}) / \mathrm{I}(\lambda \mathrm{x})$ term in 
the equation. $\mathrm{Ru}(\mathrm{bpy}) 3(\Phi=0.016)$. Deareated samples were prepared by the freeze-pump-thaw technique.

\section{Cyclic Voltammetry:}

Cyclic Voltammetry (CV) was performed in a gastight single-compartment three-electrode cell using a Voltalab 40 system from Radiometer Analytical which consists of a PGZ301 potentiostat and Voltamaster 4 software. The working electrode was a Pt-disc, the counter electrode a Pt wire, and Ag wire was used as a pseudo-reference electrode. All glassware was dried prior to use. The compounds (electrolyte, analyte and reference) were placed in a Schlenk flask that was then evacuated and heated with a heat gun to eliminate any moisture and oxygen that had entered during the addition. The flask was then evacuated and filled three times with dry nitrogen. The solvent was added via syringe directly to the sealed Schlenk flask, and then degassed for ten minutes with a gentle stream of dry $\mathrm{N}_{2}$. After degassing, the solution was added, via syringe, to the electrochemical cell under a positive $\mathrm{N}_{2}$ pressure and the electrodes then added. The solution was kept under a positive $\mathrm{N}_{2}$ pressure during the measurements but no flow was allowed through the cell. For electrochemistry of surfaces, we used $\mathrm{Ag}$ and $\mathrm{Pt}$ wires as reference and counter-electrodes respectively. The subsequent experiments were carried out using neat acetonitrile solution with tetrabutylammoniumhexafluorophosphate (Sigma Aldrich) as the electrolyte. Measurements with Pt functionalized with $\mathrm{Ru}-\mathrm{SAc}$ were performed in the same experimental conditions as mentioned above.

\section{Surface Analysis}

Atomic Force Microscopy (AFM) Imaging: AFM images of the monolayers on flat gold substrates were acquired in air at room temperature with a commercial instrument (Digital Instruments, Nanoscope IIIa, Dimension 3000, Santa Barbara, CA) operating in tapping mode. AFM images are flattened and shown without further modification. Analysis was performed using WSxM 4.0 Develop60.

Fluorescence Lifetime Microscopy (FLIM): The FLIM images and the fluorescence decays on surfaces were recorded using a Microtime 200 
(PicoQuant) attached to an Olympus IX 71 Microscope with a 100x Oilimmersion objective and a scanning speed of $6 \mu$ s per point at an excitation with a $440 \mathrm{~nm}$ laser (FWHM 80 ps). Fluorescent lifetimes were calculated from the whole area by the software SymphoTime (PicoQuant).

Formation of the junctions: Ultraflat Au surfaces were formed by a templatestripping (TS) procedure. On silicon wafers with their native $\mathrm{SiO}_{2}$ layer present a layer of $500 \mathrm{~nm}$ of Au was thermally deposited by electron-beam (e-beam) at $2-3 \times 10-6$ Torr at a rate of 8-10 $\AA$ /s. Glass slides, which were cleaned by washing with EtOH and oxygen plasma oxidation for $5 \mathrm{~min}$, of typically $1 \mathrm{~cm}^{2}$ were glued at the Ag-surface using an optical adhesive (Norland, No. 61). The optical adhesive was cured for $2 \mathrm{~h}$ by exposure to ultraviolet light for $2 \mathrm{~h}$. The glass substrates were cleaved off the Si-waver by using a razor blade after which the TS Au substrates were immersed in a solution of $2 \mathrm{mM}$ of Ru-SAc in ethanol/acetonitrile for $24 \mathrm{~h}$ at RT. After SAM formation the samples were rinsed with EtOH. Conical shaped eutectic indium-gallium (EGaIn, 75.5\% Ga $24.5 \%$ by weight, $15.7{ }^{\circ} \mathrm{C}$ melting point) alloy as top-electrodes. ${ }^{55}$ The EGaIn is a non-Newtonian fluid. On the micrometer scale, EGaIn behaves as a solid, but when sheer-pressure is applied EGaIn behaves as a liquid. The EGaIn will flow until the sheer-pressure is relieved. This behavior allows, unlike mercury, to shape the EGaIn into non-spherical shapes.

A drop of EGaIn hanging at a 26S-guage needle was brought into contact with a surface that is wettable by EGaIn, such as PDMS, glass, or Ag surfaces. The EGaIn adheres to both the surface and to the needle. Slowly retracting the needle from the EGaIn-drop, by using a micromanipulator, deforms the EGaIn drop in such a way that two conical-shaped EGaIn structures connected head-to-head arise. Further retraction of the needle results into separation of the conical shaped EGaIn structures, one at the needle and one at the surface. Subsequently, the substrate was discarded and replaced by a TS Ag surface with the SAM of interest and the conically-shaped EGaIn at the needle was brought into contact with the SAM. 


\section{References}

1. Dong, T. Y.; Huang, C. L.; Chen, C. P.; Lin, M. C., J. Organomet. Chem. 2007, 692, 5147.

2. Love, J. C.; Estroff, L. A.; Kriebel, J. K.; Nuzzo, R. G.; Whitesides, G. M., Chem. Rev. 2005, 105, 1103.

3. Ulman, A., Chem. Rev. 1996, 96, 1533.

4. Aviram, A.; Ratner, M. A., Chem. Phys. Lett. 1974, 29, 277.

5. Chen, F.; Hihath, J.; Huang, Z.; Li, X.; Tao, N. J., Annu. Rev. Phys. Chem. 2007, 58, 535.

6. Dichtel, W. R.; Heath, J. R.; Fraser Stoddart, J., Phil. Trans. R. Soc. A 2007, 365, 1607.

7. Huang, Z. F.; Chen, F.; Bennett, P. A.; Tao, N. J., J. Am. Chem. Soc. 2007, 129, 13225.

8. Joachim, C.; Gimzewski, J. K.; Aviram, A., Nature 2000, 408, 541.

9. Mativetsky, J. M.; Pace, G.; Elbing, M.; Rampi, M. A.; Mayor, M.; Samori, P., J. Am. Chem. Soc. 2008, 130, 9192.

10. Reichert, J.; Ochs, R.; Beckmann, D.; Weber, H. B.; Mayor, M.; Löhneysen, H. v., Phys. Rev. Lett. 2002, 88, 176804.

11. Mirkin, C. A.; Ratner, M. A., Annu. Rev. Phys. Chem. 1992, 43, 719.

12. Bertoncello, P.; Dennany, L.; Forster, R. J.; Unwin, P. R., Anal. Chem. 2007, 79, 7549.

13. Figgemeier, E.; Constable, E. C.; Housecroft, C. E.; Zimmermann, Y. C., Langmuir 2004, 20, 9242.

14. Obeng, Y. S.; Bard, A. J., Langmuir 1991, 7, 195.

15. Silva, M. J. J. P.; Bertoncello, P.; Daskalakis, N. N.; Spencer, N.; Kariuki, B. M.; Unwin, P. R.; Pikramenou, Z., Supramol. Chem. 2007, 19, 115

16. Zhang, X.; Bard, A. J., J. Phys. Chem. 1988, 92, 5566.

17. Albrecht, T.; Guckian, A.; Ulstrup, J.; Vos, J. G., Nano Lett. 2005, 5, 1451.

18. Cattabriga, M.; Ferri, V.; Tran, E.; Galloni, P.; Rampi, M. A., Inorg. Chim. Acta 2007, 360, 1095. 
19. Forster, R. J.; Figgemeier, E.; Lees, A. C.; Hjelm, J.; Vos, J. G., Langmuir 2000, 16, 7867.

20. Forster, R. J.; Figgemeier, E.; Loughman, P.; Lees, A.; Hjelm, J.; Vos, J. G., Langmuir 2000, 16, 7871.

21. Haga, M.-a.; Kobayashi, K.; Terada, K., Coord. Chem. Rev. 2007, 251, 2688.

22. Haga, M.-A.; Wang, K.; Kato, N.; Monjushiro, H., Mol. Cryst. and Liq. Cryst. 1999, 337, 89

23. Joe Otsuki; Tokimoto, T.; Yano, Y. N. T.; Hasegawa, T.; Chen, X.; Okamoto, Y., Chem. Eur. J. 2007, 13, 2311.

24. Meyer, T. J.; Meyer, G. J.; Pfennig, B. W.; Schoonover, J. R.; Timpson, C. J.; Wall, J. F.; Kobusch, C.; Chen, X.; Peek, B. M.; et al., Inorg. Chem. 1994, 33, 3952.

25. Nunzio Tuccitto; Vanna Torrisi; Marco Cavazzini; Tamara Morotti; Fausto Puntoriero; Silvio Quici; Sebastiano Campagna, A. L., ChemPhysChem 2007, 8, 227.

26. Terada, K.; Kobayashi, K.; Haga, M.-a., Dalton Trans. 2008, 4846.

27. Weiss, E. A.; Kriebel, J. K.; Rampi, M. A.; Whitesides, G. M., Pbil. Trans. R. Soc. A 2007, 365, 1509.

28. Aldachi, C. B., M. A.; Forrest, M. R.; Thompson, M. E., Appl Phys Lett 2000, 77, 904.

29. Thompson, M., Mater. Res. Bull. 2007, 32, 694.

30. Yersin, H., In Highly Efficient OLEDs with Phosphorescent Materials, WileyVCH Verlag GmbH \& Co. KGaA, : Weinheim, Germany, 2008; p 438.

31. Fox, M. A.; Whitesell, J. K.; McKerrow, A. J., Langmuir 1998, 14, 816.

32. Kitagawa, T.; Idomoto, Y.; Matsubara, H.; Hobara, D.; Kakiuchi, T.; Okazaki, T.; Komatsu, K., J. Org. Chem. 2006, 71, 1362.

33. Park, J. S.; Vo, A. N.; Barriet, D.; Shon, Y. S.; Lee, T. R., Langmuir 2005, 21, 2902.

34. Galoppini, E.; Guo, W.; Qu, P.; Meyer, G. J., J. Am. Chem. Soc. 2001, 123, 4342.

35. Hoertz, P. G.; Carlisle, R. A.; Meyer, G. J.; Wang, D.; Piotrowiak, P.; Galoppini, E., Nano Lett. 2003, 3, 325. 
36. Ichimura, A. S.; Lew, W.; Allara, D. L., Langmuir 2008, 24, 2487.

37. Myahkostupov, M.; Piotrowiak, P.; Wang, D.; Galoppini, E., J. Phys. Chem. C 2007, 111, 2827.

38. Nikitin, K.; Lestini, E.; Lazzari, M.; Altobello, S.; Fitzmaurice, D., Langmuir 2007, 23, 12147.

39. Weidner, T.; Kramer, A.; Bruhn, C.; Zharnikov, M.; Shaporenko, A.; Siemeling, U.; Trager, F., Dalton Trans. 2006, 2767.

40. Huang, W. Y.; Gao, W.; Kwei, T. K.; Okamoto, Y., Macromolecules 2001, 34, 1570 .

41. Issberner, J.; Vogtle, F.; De Cola, L.; Balzani, V., Chem. Eur. J. 1997, 3 , 706.

42. Campagna, S.; Puntoriero, F.; Nastasi, F.; Bergamini, G.; Balzani, V., Top. Curr. Chem. 2007, 280, 117.

43. Juris, A. B., V; Barigelletti,F; Campagna, S; Belser, P; von Zelewsky, A., Coord. Chem. Rev. 1982, 84, 85.

44. Weiss, E. A.; Chiechi, R. C.; Kaufman, G. K.; Kriebel, J. K.; Li, Z.; Duati, M.; Rampi, M. A.; Whitesides, G. M., J. Am. Chem. Soc. 2007, 129, 4336.

45. Jian, H.; Tour, J. M., J. Org. Chem. 2003, 68, 5091.

46. Wei, S.; Gafney, H. D.; Clark, J. B.; Perettie, D. J., Chem. Phys. Lett. 1983, 99, 253.

47. D'Aleo, A.; Williams, R. M.; Chriqui, Y.; Vijay M. Iyer; Belser, P.; Vergeer, F.; Ruiz, V.; Unwin, P. R.; Cola, L. D., The Open Inorganic Chemistry Journal 2007, 1, 26.

48. Pramod, P.; Sudeep, P. K.; Thomas, K. G.; Kamat, P. V., J. Phys. Chem. B 2006, 110, 20737.

49. Bertoncello, P.; Kefalas, E. T.; Pikramenou, Z.; Unwin, P. R.; Forster, R. J., J. Phys. Chem. B 2006, 110, 10063.

50. Kalyanasundaram, K., Coord. Chem. Rev. 1982, 46, 159.

51. Ricci, A.; Rolli, C.; Rothacher, S.; Baraldo, L.; Bonazzola, C.; Calvo, E.; Tognalli, N.; Fainstein, A., J. Solid State Electrochem. 2007, 11, 1511.

52. Kondo, T.; Kanai, T.; Uosaki, K., Langmuir 2001, 17, 6317.

53. Laviron, E., J. Electroanal. Chem. 1979, 101, 19.

54. Laviron, E.; Roullier, L., J. Electroanal. Chem. 1980, 115, 65. 
55. Chiechi, Ryan C.; Weiss, Emily A.; Dickey, Michael D.; Whitesides, George M., Angew. Chem. Int. Ed. 2008, 47, 142.

56. Dickey , M. D.; C; C. R.; Larsen, R. J.; Weiss, E. A.; Weitz, D. A.; Whitesides, G. M., Adv. Funct. Mater. 2008, 18, 1097.

57. Tran, E.; Rampi, M. A.; Whitesides, G. M., Angew. Chem. Int. Ed. 2004, 43, 3835 . 
Chapter 4 


\title{
CHAPTER 5
}

\section{PHOTOINDUCED CHARGE TRANSFER BETWEEN RUTHENIUM TRIPODS AND CdTe QUANTUM DOTS}

\begin{abstract}
A tripodal ruthenium complex with thiol functionality has been attached to green-emitting CdTe quantum dots to form a photoactive nanoscale system that shows electron transfer $\left(k_{\text {et }}=2.5 \times 10^{9} \mathrm{~s}^{-1}\right)$ from the CdTe particle to the Ru-complex. Subpicosecond as well as nanosecond transient absorption measurements are presented in this chapter. Control experiments with a ruthenium complex with similar structure that lacks any terminal binding groups demonstrated much slower electron transfer dynamics and change in the absorption characteristics, thus providing indirect evidence for binding of the thiol-terminated complex.
\end{abstract}




\section{Introduction}

In the past two decades, a significant amount of work has been devoted to the study of nanocrystal quantum dots (NQDs) owing to their size-dependent properties coupled with high photostability and high luminescence $e^{1-4}$. These characteristics make quantum dots potential candidates for numerous applications including photovoltaics ${ }^{5-8}$, LEDs $^{9-11}$, imaging ${ }^{12-13}$, lasers ${ }^{14-15}$, and sensors. ${ }^{16-17}$ For practical applications, such NQDs have to be processable and hence require appropriate functionalization. In this regard, several groups have reported the anchoring of photoactive units onto CdSe and CdTe quantum $\operatorname{dots}^{18-21}$. In particular, the use of metal complexes such as $\operatorname{Ru}(\mathrm{II}), \operatorname{Ir}(\mathrm{III})$, and Os(II) in combination with quantum dots is of interest due to the attractive photophysical properties of the metal complexes such as very long excitedstate lifetimes coupled with rich electrochemistry. ${ }^{22-25}$ Such nanoassemblies combine two different classes of materials with contrasting optical properties. To be specific, the quantum dots have very high oscillator strengths, short radiative lifetimes of about $20 \mathrm{~ns}$, and a very narrow emission profile. There is minimal vibrational broadening in the emission spectra of the quantum dots, and thereby the ground state of the nanocrystals proves to be an almost ideal two-level excitation system. On the other hand, the metal complexes possess relatively weak oscillator strengths, longer excited-state lifetimes of about 1 to $2 \mu$ s and broad emission spectrum due to the charge transfer character of the emission. For photovoltaic applications, suitable candidates have to be employed to successfully harvest the excitons generated in the nanocrystals. In this regard, the metal complexes serve as an interesting option due to their emission and redox properties, with energy and charge transfer in such nanoconjugates being of quintessential interest to hybrid solar cells. However, to date only a few reports are available with metal complex/quantum dot conjugates. $^{13,}$ 26-29 In particular, there are very few reports on ruthenium polypyridyl complex, in combination with $\mathrm{CdSe} .^{26-27,30-33}$. In one such paper the authors anchor the ruthenium chromophores onto the electron-acceptor quantum dots via carboxylate groups. Furthermore, they report a photoinduced hole transfer from quantum dot to the ruthenium complex which occurs on a very fast time scale of 5 ps. In view of the stability of these 
nanocomposites, it would be very useful to have multiple anchoring groups present on the ruthenium polypyridyl core. Galoppini and coworkers have reported a series of ruthenium molecules with rigid tripodal structure with three carboxylate groups and their assembly on $\mathrm{TiO}_{2}$ films and have demonstrated a photoinduced electron transfer from the ruthenium core to the nanocrystalline surface. ${ }^{34-35}$ They have demonstrated that by employing stable geometries of different tripodal lengths, the electron transfer and successive charge recombination rate could be extended to as long as few hundreds of microseconds.

In the current chapter, photophysical studies carried out on nanoassemblies based on green emitting CdTe quantum dots and tripodal ruthenium complexes are reported. CdTe nanocrystals were chosen for this study since the conduction band of CdTe is much higher in energy as compared to CdSe relative to vacuum. This would enable the quantum dot to be the electron donor. Further, the preference of thiol functionality over the carboxylic group stems from the stronger affinity of thiols to $\mathrm{CdTe}^{36}$ as compared to CdSe. Upon exciting the quantum dots at the exciton band gap energy, pump induced electron transfer from the conduction band of CdTe to the ruthenium molecule is observed which is characterized by the time resolved measurements in the subpicosecond regime. 


\section{Results and Discussion}

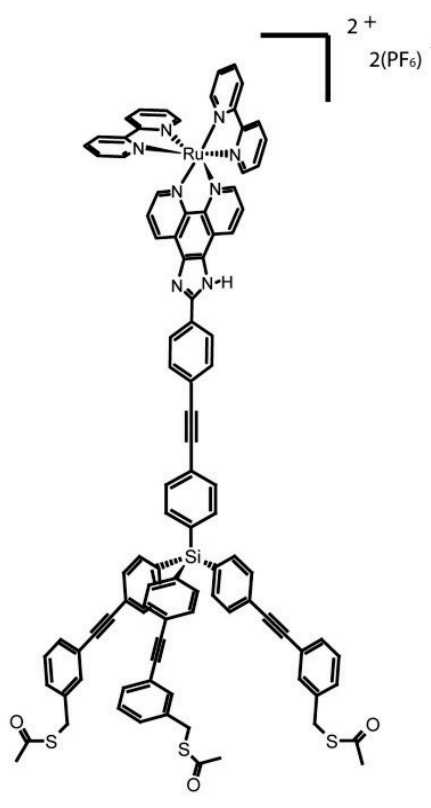

Ru -SAC

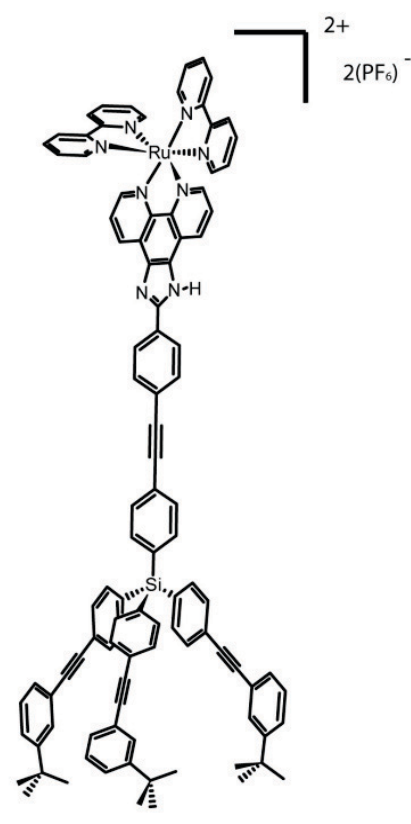

$\mathrm{Ru}$-tert-Bu

Chart 5.1: Schematic formulas of complexes employed in the experiments and their abbreviations.

\section{CdTe-ruthenium tripod nanoassemblies}

The tripodal complexes based on $\mathrm{Ru}(\mathrm{bpy})_{2}$ (phen) chromophores (where bpy $=$ 2,2' bipyridine and phen $=(2-(4-(2$-ethynyl)phenyl) $-1 \mathrm{H}$-imidazo $[4,5-f][1,10]$ phenanthroline)) are shown in Chart 5.1. Ru-SAc possesses three acetylthiol groups that were used to anchor the metal complex to the surface of the quantum dot. The nature of binding between QDs and Ru-SAc could be possibly explained on similar grounds as described by Guyot-Sionnest and coworkers ${ }^{37}$ by employing Pearson's 'hard and soft acid base' (HSAB) concept. The quantum dot surface contains $\mathrm{Cd}^{2+}$ ions which are soft acids due to their large polarizable core and hence they have a stronger affinity to softer bases. Generally alkyl thiolates (RS), which are soft bases, have the strongest affinity to the quantum dot surface through the lone pair of electrons on sulfur. However, in case of Ru-SAc, there are two heteroatoms, sulfur and oxygen, 
with lone pair of electrons which could bind to the surface, although the exact binding site is not clear at this point. Ru-tert-Bu was used as a reference compound because it lacks the anchoring groups, and therefore, a weaker interaction is expected with the quantum dots. The nanoassemblies were prepared as explained in the experimental section. All experiments were carried out under identical conditions (same excitation wavelength, slit width, integration time) and the absorption of ruthenium moieties and quantum dots were always the same in the reference solutions and in the assemblies respectively. Since the concentrations of both ruthenium complexes and the quantum dots were very low, the isolation of the nanoassemblies was not possible.

\section{Photophysical studies of the nanoassembly}

The absorption spectra of CdTe, Ru-SAc and CdTe-Ru-SAc are displayed in figure 5.1. The CdTe nanocrystals used in this work have an absorption band around $500 \mathrm{~nm}$ originating from the $1 \mathrm{~S}_{1 / 2}(\mathrm{e})-1 \mathrm{~S}_{3 / 2}(\mathrm{~h})$ excitonic transition (assuming the absence of any band mixing effects). A shoulder around $320 \mathrm{~nm}$ is observed which reflects transitions into higher quasi-continuum states involving $1 \mathrm{P}$ and $1 \mathrm{D}$ states of the CdTe nanocrystals. The lowest energy absorption band of Ru-SAc has a maximum at $465 \mathrm{~nm}$ and is characteristic of singlet metal-to-ligand charge transfer $\left({ }^{1} \mathrm{MLCT}\right)$ transitions involving the bipyridine (bpy) ligands, $\mathrm{M} \mathrm{d}(\pi) \rightarrow \mathrm{bpy}\left(\pi^{*}\right)$, while the higher energy MLCT bands involve the (2-(4-(2-ethynyl)phenyl)-1H-imidazo[4,5-f $[1,10]$ phenanthroline) ligands. The bands at 300-350 $\mathrm{nm}$ are assigned to $\pi \rightarrow \pi^{*}$ transitions on the phenylene ethylene unit (at $350 \mathrm{~nm}$ ) and to the bpy and phen ligands at higher energy. A similar spectrum is recorded for Ru-tert-Bu as well. A more detailed photophysical study of the Ru-SAc and Ru-tert-Bu are presented in chapter 4.

In case of the absorption of the nanoassembly, we observe contributions from both, the MLCT absorption of Ru-SAc and interband absorption of the quantum dots. However, it is important to note that the spectrum of the nanoassembly is not simply a linear combination of the absorption spectra of the individual components (Figure 5.1, inset) as expected in case of two non- 
interacting chromophores. In fact, we observe for CdTe-Ru-SAc that the MLCT absorptions undergo a bathochromic shift of about $4 \mathrm{~nm}$ (Figure 5.1 inset) compared to the calculated spectrum obtained by the sum of the Ru-SAc and CdTe absorption spectra.

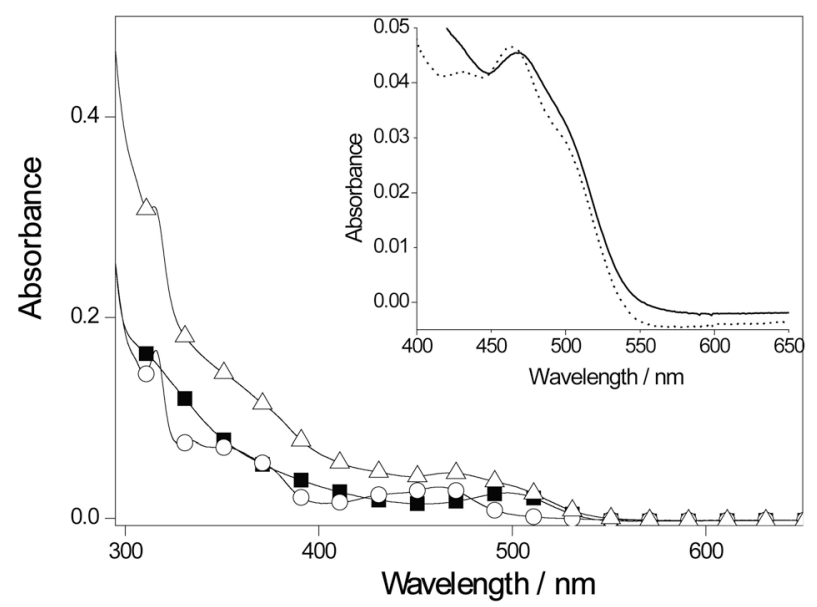

Fig. 5.1: UV -Visible Absorption spectra of CdTe (--), Ru-SAc (-०-) and CdTe/RuSAc $\left.(-\rangle_{-}\right)$. All the spectra are recorded in degassed $1: 1 \mathrm{v} / \mathrm{v}$ benzonitrile / toluene solution. Inset: Comparison between numerical sum of CdTe and Ru-SAc (*.) and experimentally observed nanoassembly spectrum $(-)$

This shift is not observed when the reference compound Ru-tert-Bu is mixed with the quantum dots (see figure 5.2 inset). Furthermore, the deconvolution of the spectra indicates that the shift is not due to the superimposition of the two bands that can cause an apparent shift of the ${ }^{1}$ MLCT band. Therefore, we believe that the low energy shift is due to a change in the LUMO energy of the lowest excited state involved in the MLCT. In the presence of the quantum dots, which have electron deficient surface sites, the electron density on the phenanthroline derivative decreases, rendering this unit a better electron acceptor, and therefore switching the MLCT character from bpy to the phenanthroline derivative.

Ru-tert-Bu shows as similar absorption spectrum as that of Ru-SAc and the assignments of the transitions are identical to Ru-SAc. On the other hand, the above mentioned red shift is not observed in the control experiment, for the 
mixture of the reference compound Ru-tert-Bu with the quantum dots under identical conditions (Figure 5.2). However, the absorbance of quantum dots differs slightly as can be seen from the inset of Figure 5.2, which may result from a slightly higher baseline of the assembly due to scattering on the colloidal nanocrystals.

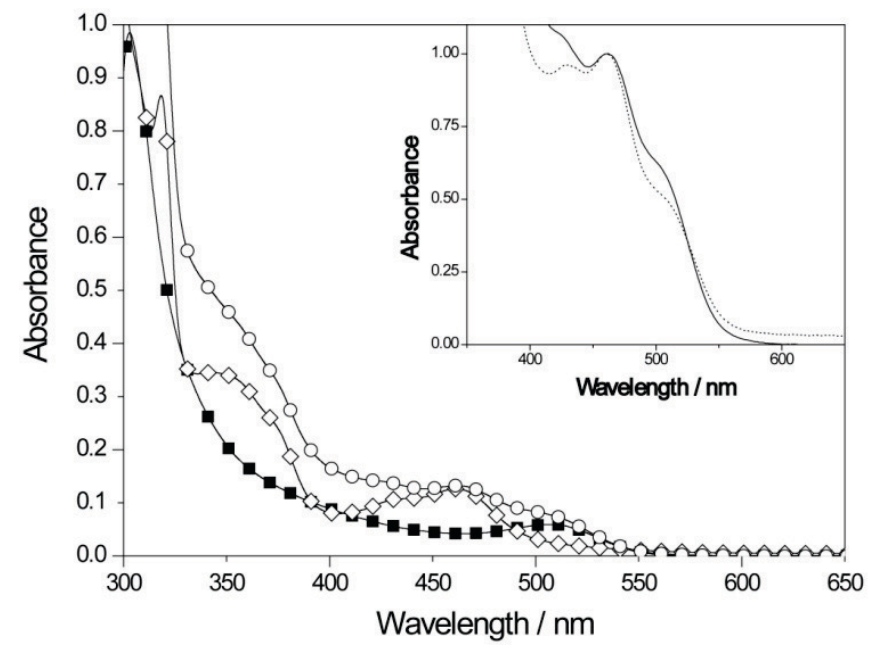

Fig. 5.2: UV -Visible Absorption spectra of CdTe (--), Ru-tert-Bu (-०-) and $\mathrm{CdTe} /$ Ru-tert-Bu $\left(-\diamond_{-}\right)$. All the spectra are recorded in degassed 1:1 v/v benzonitrile / toluene solution. Inset: Comparison between numerical sum of CdTe and Ru-SAc (*) and experimentally observed nanoassembly spectrum $(-)$

The emission spectra of the compounds and the assembly are shown in Figure 5.3. All the spectra were measured in $1: 1 \mathrm{v} / \mathrm{v}$ benzonitrile/toluene solution at an excitation wavelength of $500 \mathrm{~nm}$. Quantum dots have a narrow emission band with maximum at $530 \mathrm{~nm}$. The fact that the emission maximum lies very close to the absorption onset $(500 \mathrm{~nm})$ indicates that the emission originates from the direct recombination of the charge carriers. Ru-SAc on the other hand has a very broad emission feature centered around $610 \mathrm{~nm}$; this large Stokes' shift is due to the triplet character of the emissive state which is assigned as ${ }^{3} \mathrm{MLCT}$. It is very evident from the emission spectrum of the assembly that emission of both CdTe and Ru-SAc are quenched drastically after 1 hour of mixing these solutions at room temperature (see Figure 5.3). 
The emission of quantum dots is quenched virtually completely and ruthenium emission is reduced by about $70 \%$.

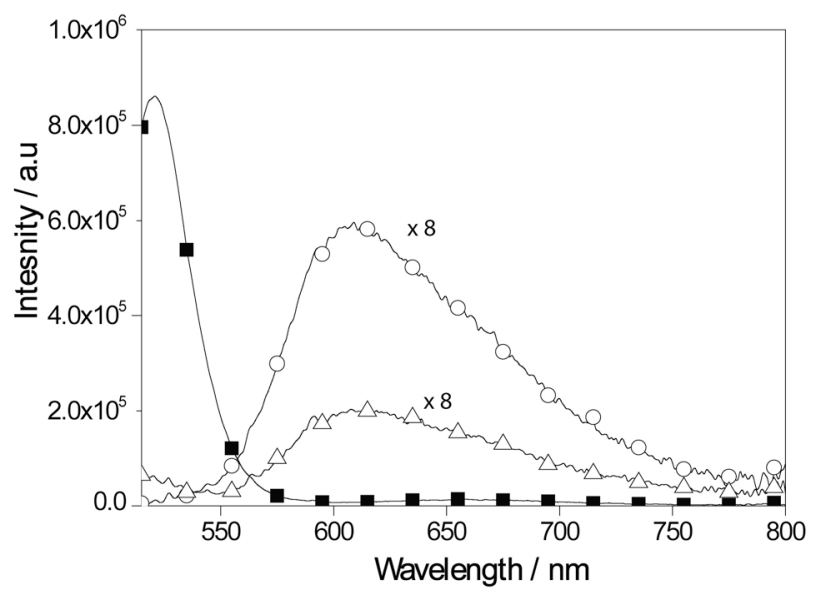

Fig.5.3: Room temperature emission spectra of CdTe (-m-), Ru-SAc (-o-) and $\mathrm{CdTe} / \mathrm{Ru}-\mathrm{SAc}(-\Delta-)$. All the spectra are recorded in degassed 1:1 v/v Benzonitrile / toluene solution. $\lambda_{\mathrm{ex}}=500 \mathrm{~nm}$

Even in the control experiment with Ru-tert-Bu (Figure 5.4), the ruthenium complex is moderately quenched while the nanocrystal emission is affected to a much greater extent and is quenched by $75 \%$. However, the extent of quenching of both the components is lesser than compared to Ru-SAc. The reason behind this finding might again be traced back to the lack of anchoring groups resulting in weaker (non-covalent) interactions between ruthenium complex and the nanoparticles. To gain more insight into these emission quenching effects time resolved measurements were performed. 


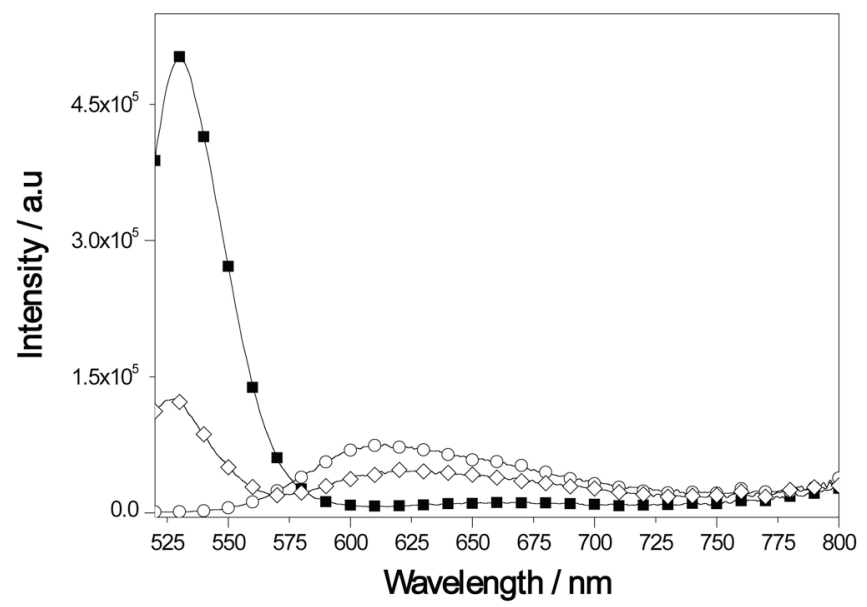

5.4: Room temperature emission spectra of $\mathrm{CdTe}(--)$, Ru-tert-Bu (-०-) and $\mathrm{CdTe} / \mathrm{Ru}-$ tert- $\mathrm{Bu}\left(-\vartheta_{-}\right)$. All the spectra are recorded in degassed 1:1 v/v Benzonitrile / toluene solution. $\lambda_{\mathrm{ex}}=500 \mathrm{~nm}$

The excited-state lifetimes were measured with a time-correlated-singlephoton-counting (TCSPC) system using a $402 \mathrm{~nm}$ excitation and monitoring various emission wavelengths (Table 5.1). Quantum dots usually exhibit multiexponential decays that can be attributed to the size distribution, trap states and defect emission associated with them ${ }^{38-39}$. In general, they have very short radiative lifetimes of less than 20 ns at room temperature. The green emitting CdTe nanocrystals used in this work exhibit multiexponential excited state lifetimes dominated by 5 ns and 15 ns components. Ru-SAc and Ru-tert$\mathrm{Bu}$ on the contrary have a longer lifetime of 1 microsecond in deaerated conditions. In the CdTe/Ru-SAc assembly, however, the emission lifetime of CdTe quantum dots decreases by about 35\% whereas Ru-SAc lifetime measured in this time regime seems to be unchanged. 


\begin{tabular}{|c|c|c|c|c|c|c|}
\hline Compound & $\boldsymbol{\tau}_{\mathbf{1}}$ & Rel. & $\boldsymbol{\tau}_{\mathbf{2}}$ & Rel. & $\boldsymbol{\tau}_{\mathbf{3}}$ & Rel. \\
\hline CdTe & $5.34^{\mathrm{a}}$ & 50 & 15.7 & 40 & 0.8 & 10 \\
\hline $\mathbf{R u}-\mathbf{S A c}$ & $1033^{\mathrm{b}}$ & 100 & - & - & - & - \\
\hline $\mathbf{C d T e} / \mathbf{R u}-\mathbf{S A c}$ & $3.5^{\mathrm{a}}$ & 45 & 12.7 & 43 & 0.55 & 12 \\
\hline & $1048^{\mathrm{b}}$ & 100 & - & - & - & - \\
\hline $\mathbf{R u}-$ tert-Bu & $1029^{\mathrm{b}}$ & 100 & - & - & - & - \\
\hline $\mathbf{C d T e} / \mathbf{R u}-$ tert- & $5.4^{\mathrm{a}}$ & 57 & 15 & 31 & 1.4 & 12 \\
\hline & $1025^{\mathrm{b}}$ & 100 & - & - & - & - \\
\hline
\end{tabular}

Table: 5.1. Excited state lifetimes of CdTe/Ru-SAc and CdTe/ Ru-tert-Bu assembly measured by time correlated single photon counting.

The extent of quenching of the ruthenium emission is however not correlated with the long excited state lifetime measured in the assembly. It is believed that the strong quenching $(\sim 70 \%)$ results from the excited state population being dramatically reduced due to a non-radiative pathway. Indeed, the unchanged lifetime reflects the emission of ruthenium complex in solution not bound to the particles. Assuming that roughly about 4 to 5 complexes are coordinated to the CdTe clusters based on the size considerations of the quantum dots (assuming the spherical structure of the nanocrystals) and Ru-SAc, about 50\% of the complex should remain in solution. However, even for the assembly with the reference compound, $\mathrm{CdTe} / \mathrm{Ru}$-tert-Bu, quenching is observed when the quantum dots are added to the solution. Quantum dots emission gets quenched by about $75 \%$. The quenching of the non-thiolated ruthenium complex emission is estimated to be about 32\% (Figure 5.4). From the Table 5.1 , it is evident that the lifetime of quantum dots are not quenched. It is presently not clear as to why the particles and ruthenium get in a close contact in order to favor the quenching of both emissions, but previous reports show that for charged complexes adsorption on the quantum dot surface occurs even at low concentration. ${ }^{26}$ 
On the basis of our experimental results, we envisage that the quenching mechanism is a photoinduced electron transfer (PET). In systems with CdSe or $\mathrm{TiO}_{2}$ with ruthenium complex, $\mathrm{Ru}^{2+}$ usually acts as electron donor and quantum dots act as electron acceptors ${ }^{26,34}$ because of the relative positions of the HOMO levels of $\mathrm{Ru}^{2+}$ and the nanocrystal acceptors. However, with $\mathrm{CdTe}$ quantum dots, the conduction band lies at a higher energy than the HOMO levels of the ruthenium tripod and hence CdTe acts as an electron donor in the present system. The electron donating capability of $\mathrm{CdTe}$ is also reported by Guldi et al. wherein they have anchored a $\mathrm{C}_{60}$ derivative onto $\mathrm{CdTe}$ nanocrystals and report an electron transfer from NQD to the fullerene derivative. $^{18}$

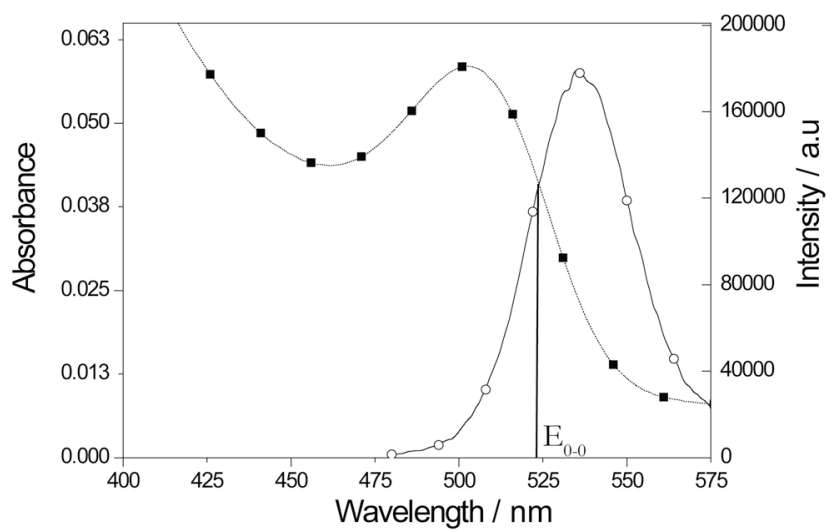

Fig.5.5: Absorption (--) and emission (-०-) of CdTe nanocrystals. The intersection of these two bands is approximated as the $\mathrm{E}_{00}$ transition for the nanocrystals

In order to thermodynamically prove that the photoinduced electron transfer process in these assemblies can occur, we need to estimate the degree of exoergonicity of such a reaction by using the simplified Rehm-Weller equation: $:^{40}$

$$
\Delta \mathrm{G}_{\mathrm{eT}}=\mathrm{e}\left(\mathrm{E}_{\mathrm{ox}}-\mathrm{E}_{\mathrm{red}}\right)-\mathrm{E}_{00}
$$

where $\mathrm{E}_{\mathrm{ox}}$ is the first oxidation potential of the donor (in this case the LUMO of the CdTe nanocrystal), $\mathrm{E}_{\text {red }}$ is the first reduction potential of the acceptor (Ru-Sac), and $\mathrm{E}_{00}$ is the $0-0$ transition of the moiety which is being excited and 
e is the elementary charge. $\mathrm{E}_{00}$ is the difference in energy between the lowest vibrational levels of the excited state and the ground state. In this case, $\mathrm{E}_{00}$ can be considered as the 0-0 transition of the CdTe nanocrystal since $90 \%$ of the incident photons are absorbed by the dots. The $0-0$ transition can be approximated as the intersection of the band edge absorption and the exciton emission. For the quantum dots used in this study, $\mathrm{E}_{00}$ is estimated to be 2.37 $\mathrm{eV}$ (Figure 5.5). The relative conduction band (CB) position of the dots is estimated, as described by Sykora et al., ${ }^{26}$ by using the effective mass approximation to be $0.658 \mathrm{~V}$ vs. SCE. The first reduction potential of Ru-SAc is $-1.33 \mathrm{~V}$ vs. SCE. ${ }^{41}$ By employing these values in the above equation, we find that the $\Delta G_{\mathrm{eT}}$, the free energy change involving the photoinduced electron transfer turns out to be exoergonic $\left(\Delta \mathrm{G}_{\mathrm{eT}}=-0.409 \mathrm{eV}\right)$.

\section{Transient absorption studies}

As mentioned above, in order to clarify if very fast processes occur which are not visible in our spectra and excited-state lifetime measurements, we have investigated the processes using subpicosecond and nanosecond transient absorption spectroscopy. Such techniques are essential to obtain information regarding the depopulation of electron $1 \mathrm{~S}$ state of NQDs. ${ }^{42-43}$ To probe the electron transfer dynamics, femtosecond transient absorption measurements were performed on the nanoassemblies as well as individual components in solution with $500 \mathrm{~nm}$ laser excitation. Figure 5.6 represents the transient absorption spectra of CdTe, CdTe-Ru-SAc and CdTe-Ru-tert-Bu recorded 4 ps after laser excitation. For the quantum dots, there are two important features present: a) a negative absorption trace at $500 \mathrm{~nm}$ and b) a high energy transient band at $445 \mathrm{~nm}$. The negative absorption trace at $500 \mathrm{~nm}$ is attributed to the $\mathrm{NQD}(\mathrm{e}, \mathrm{h})$ state mostly due to presence of the electron in the LUMO (senvelope). The presence of the hole possibly does not quench the HOMOLUMO transition, because there are many hole levels nearly degenerate (due to spin-orbit coupling). 


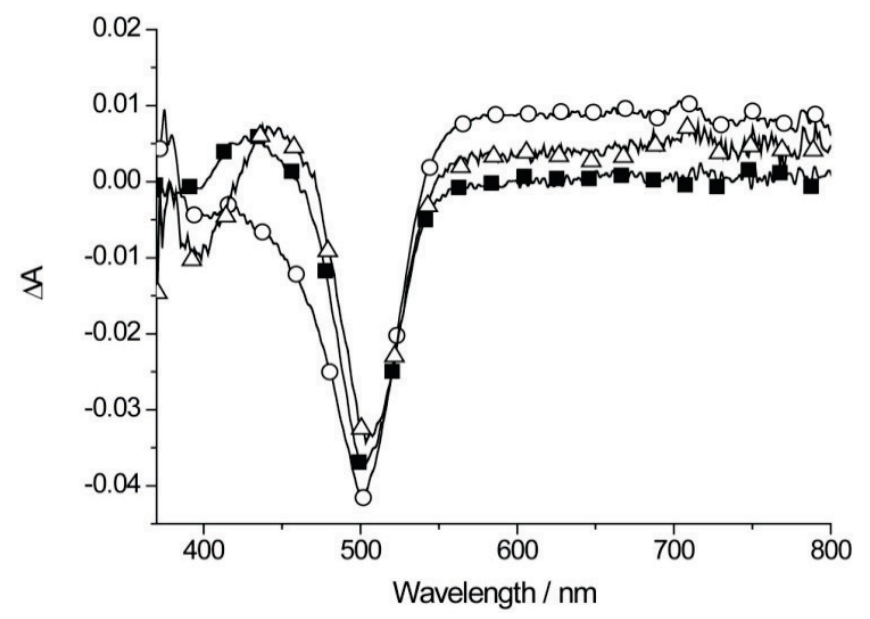

Figure 5.6: Transient absorption spectra of CdTe (---),CdTe/Ru-SAc (-O-) and $\mathrm{CdTe} / \mathrm{Ru}$-tert-Bu $(-\Delta-)$ recorded 4 ps after laser excitation

The higher energy band at $445 \mathrm{~nm}$ arises due to the state filling ${ }^{44-45}$ and Stark effects ${ }^{45}$ which are characteristic of quantum dots. The negative absorption trace at $500 \mathrm{~nm}$ has longer decay kinetics of few nanoseconds consistent with the radiative lifetime measured using TCSPC. The $445 \mathrm{~nm}$ band of the quantum dots on the other hand has extremely fast (sub-picosecond) dynamics due to the intraband relaxation. Such fast intraband dynamics have been reported for CdSe systems. ${ }^{37,46}$ The CdTe/Ru-SAc spectrum exhibits a different trace compared to CdTe after 4 ps as is evident from Figure 5.6 whereas $\mathrm{CdTe} / \mathrm{Ru}$-tert- $\mathrm{Bu}$ has similar features to that of CdTe transient spectrum. Complete time evolution of these systems on the picosecond and nanosecond timescales is explained in the next section. Figure 5.7 presents the sub-picosecond transient absorption spectra of CdTe, Ru-SAc and CdTe-RuSAc recorded at different times after $500 \mathrm{~nm}$ laser excitation. 

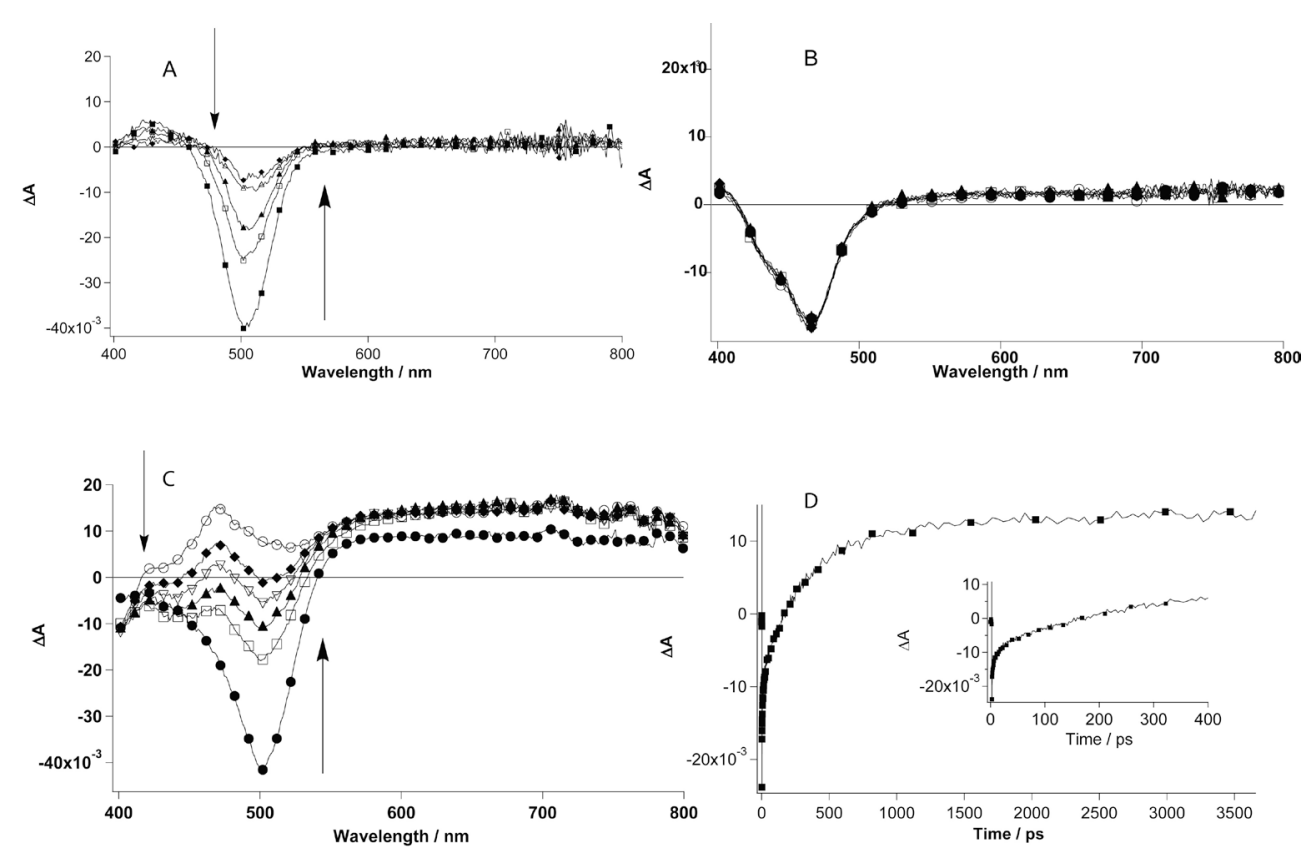

Fig. 5.5. Femtosecond transient absorption spectra of (A) CdTe, (B) Ru-SAc and (C)

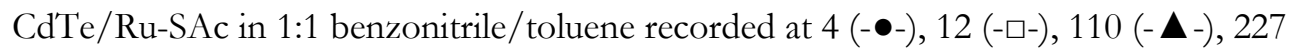
$(-\nabla), 402(--)$ and $3660(-0-)$ ps after the laser pulse. $\lambda_{\text {ex }}=500 \mathrm{~nm}$. (D) Kinetics monitored at $465 \mathrm{~nm}$ with lifetimes $\tau_{1}=22 \mathrm{ps}(15 \%)$ and $\tau_{2}=375 \mathrm{ps}(85 \%)$. Inset:

Kinetics at early time

Immediately after the pulse, two negative absorption features, one very weak at $445 \mathrm{~nm}$ and another relatively strong band at $500 \mathrm{~nm}$, and a broad positive band in the red region of the spectrum due to the radical anion of the highly conjugated paraphenylene-ethylene units of the tripodal base are observed. The negative absorption features can be attributed to the formation of both excited states: the depopulation of the $1 S_{3 / 2}(\mathrm{~h})$ state for the NQDs $(500 \mathrm{~nm})$, and to a much lesser extent due the excitation wavelength, the disappearance of the ${ }^{1}$ MLCT for the ruthenium complex $(450 \mathrm{~nm})$. While the bleaching at $500 \mathrm{~nm}$ recovers with time, we noticed that the band at $445 \mathrm{~nm}$ slightly increases in intensity in a few picoseconds, suggesting a possible energy transfer from the excited NQDs to the ruthenium moieties. The spectrum evolves with the formation, in about 400 ps, of a transient feature at about 465 
$\mathrm{nm}$, which can be attributed to the oxidized CdTe particles since the radical cation of these NPs has already been reported. ${ }^{18}$

Both, the formation and decay kinetics have been calculated at the two wavelengths where the major changes occur (Figure $5.5 \mathrm{D}$ ). The data show that the $500 \mathrm{~nm}$ negative absorption feature recovers with the same kinetics as the formation of the new positive bands at $465 \mathrm{~nm}$. The complexity of the decays of the quantum dots are also mirrored in the multiexponential kinetics measured by transient spectroscopy. All the processes however are much faster compared to the individual components since the quantum dots have excited-state lifetimes of about 5 to $15 \mathrm{~ns}$ and $\mathrm{Ru}-\mathrm{SAc}$ has about 1 microsecond excited-state lifetime due to the charge-transfer process.

The difference in the behavior of individual components and the CdTe/RuSAc can be easily seen in Figure 5.6 that shows the transient absorption traces of CdTe, Ru-SAc and the assemblies monitored after 402 ps. It is obvious that the spectra of the components do not correspond to the spectrum of the assembly since, as already discussed, the excited state of the NQDs and ruthenium in the assembly have very different kinetics and a new feature at 465 $\mathrm{nm}$, which is observed only in the assembly.

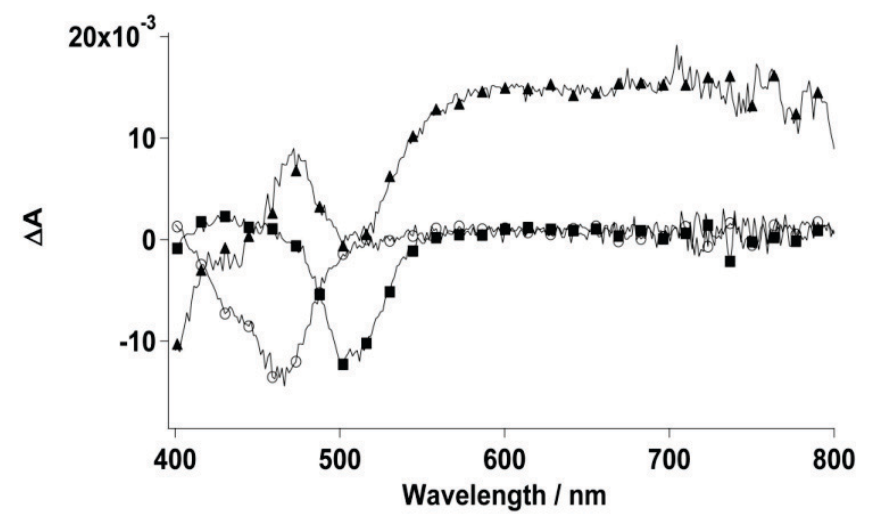

Fig. 5.6: Comparison of transient absorption spectra of CdTe (--), Ru-SAc (-०-) and $\mathrm{CdTe} / \mathrm{Ru}-\mathrm{SAc}(-\mathbf{\Delta}-) 402 \mathrm{ps}$ after of laser excitation. 
The broad feature between $550 \mathrm{~nm}$ to $800 \mathrm{~nm}$ is absent in the spectra of CdTe alone and Ru-SAc alone. In addition, the band is formed instantaneously indicating that this feature is due to the excited state of the CdTe-Ru-SAc assembly. As indicated above, since CdTe is the electron donor and the Ru tripod is the acceptor, this broad band could be attributed to the electron delocalized on the $p$-phenylene ethylene moiety. Similar transient features have been reported by our group for photoinduced electron transfer in bimetallic complexes involving $\mathrm{Ru}(\mathrm{II})$ and $\mathrm{Os}(\mathrm{II})$ bridged by polyphenyls, which substantiates the present assignment. ${ }^{47}$
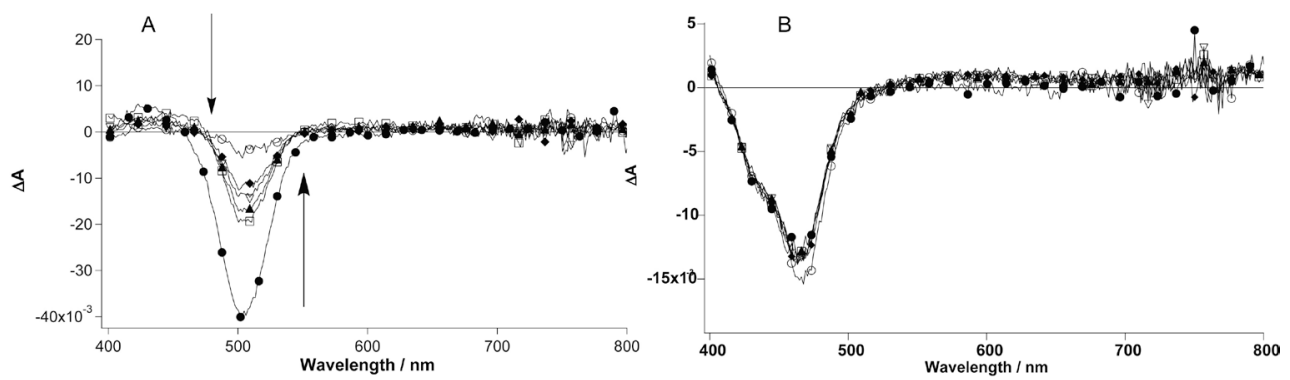

C
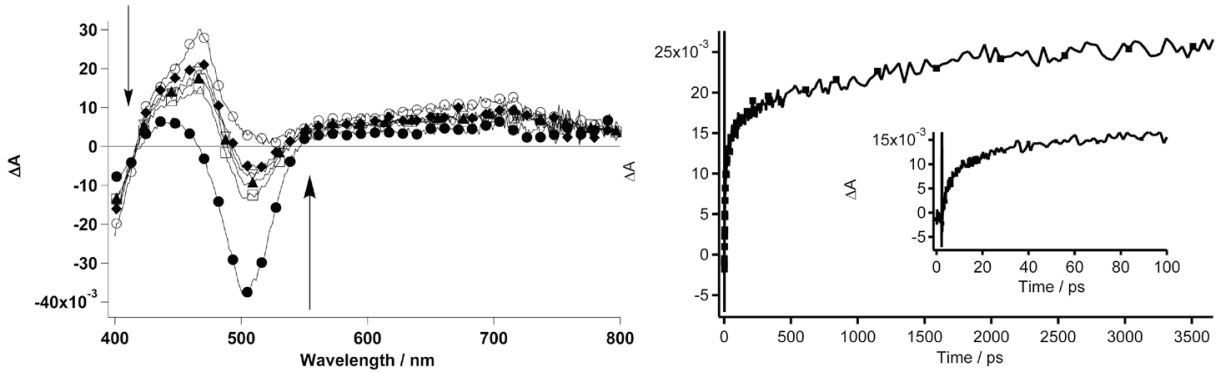

Fig. 5.7: Femtosecond transient absorption spectra of (A) CdTe, (B) Ru-tert-Bu, (C) $\mathrm{CdTe} / \mathrm{Ru}-$ tert-Bu in 1:1 benzonitrile/toluene recorded at $4(-\bullet-), 12(-\square-), 110(-\mathbf{\Delta}-)$, $227(-\nabla), 402(--)$ and $3660(-0-)$ ps after the laser pulse. $\lambda_{\text {ex }}=500 \mathrm{~nm}$. (D) Kinetics monitored at $461 \mathrm{~nm}$ with lifetimes $\tau_{1}=72 \mathrm{ps}(20 \%)$ and $\tau_{2}=1364 \mathrm{ps}(80 \%)$.

Inset:Kinetics at $463 \mathrm{~nm}$ at early times. 
We carried out a control experiment with the reference complex Ru-tert-Bu and CdTe quantum dots under identical conditions, wherein the absence of the anchoring groups would presumably result in a weaker interaction (if any) with the nanocrystals. Even in this case we found similar but not identical spectral features (Figure 5.7) indicating that electron transfer is possible in the case of a ruthenium complex lacking anchoring groups. We suggest that an electrostatic interaction is playing an important role in forming a non-covalent assembly between the Ru-tert-Bu and CdTe quantum dots. As expected, the photoinduced electron transfer from $\mathrm{CdTe}$ to $\mathrm{Ru}$-tert-Bu is slower than that in the covalently linked complex due to a weaker electronic interaction, which is evident by the formation of the charge separated species in about 1 ns (see Figure 5.6). The absence of $445 \mathrm{~nm}$ component indicates that no energy transfer process can be detected in the non-covalently bound system. In the case of $\mathrm{CdTe} / \mathrm{Ru}-\mathrm{SAc}$, another interesting feature is the positive transient feature at $445 \mathrm{~nm}$ which is present in CdTe reference spectrum is missing (Figure 5.5, panel C). The possible explanation could be that the charges at the higher energy levels in the conduction band of CdTe are effectively trapped by Ru-SAc. A similar effect is documented for CdSe NQDs wherein thiols trap holes very effectively and the fast (sub picosecond) intraband relaxation is extended to tens of picoseconds and the relaxation occurs via Auger recombination. ${ }^{14,37,46}$ We see a similar effect in our system, however, the relaxation of these 'hot carriers' occurs via an energy transfer to the ruthenium moiety which is evident by the appearance of a negative absorption band at $445 \mathrm{~nm}$ after few picoseconds. In the control experiment, such effective charge trapping is not possible, and hence at the early times, this $445 \mathrm{~nm}$ positive transient band is visible but is subsequently masked by a much stronger $465 \mathrm{~nm}$ electron transfer band.

In order to study the back electron transfer that does not occur in the few nanoseconds available by the femtosecond set-up we have investigated the transient spectra in the nanosecond range. Figure 5.8 depicts the nanosecond transient spectra for the CdTe, Ru-SAc and CdTe-Ru-SAc recorded with a 500 $\mathrm{nm}$ laser pulse excitation at different delays. As shown in Figure 5.8, both the 
$465 \mathrm{~nm}$ and $550-800 \mathrm{~nm}$ features follow the same decay kinetics. They both decay in about 325 ns which correspond to the back electron transfer process.
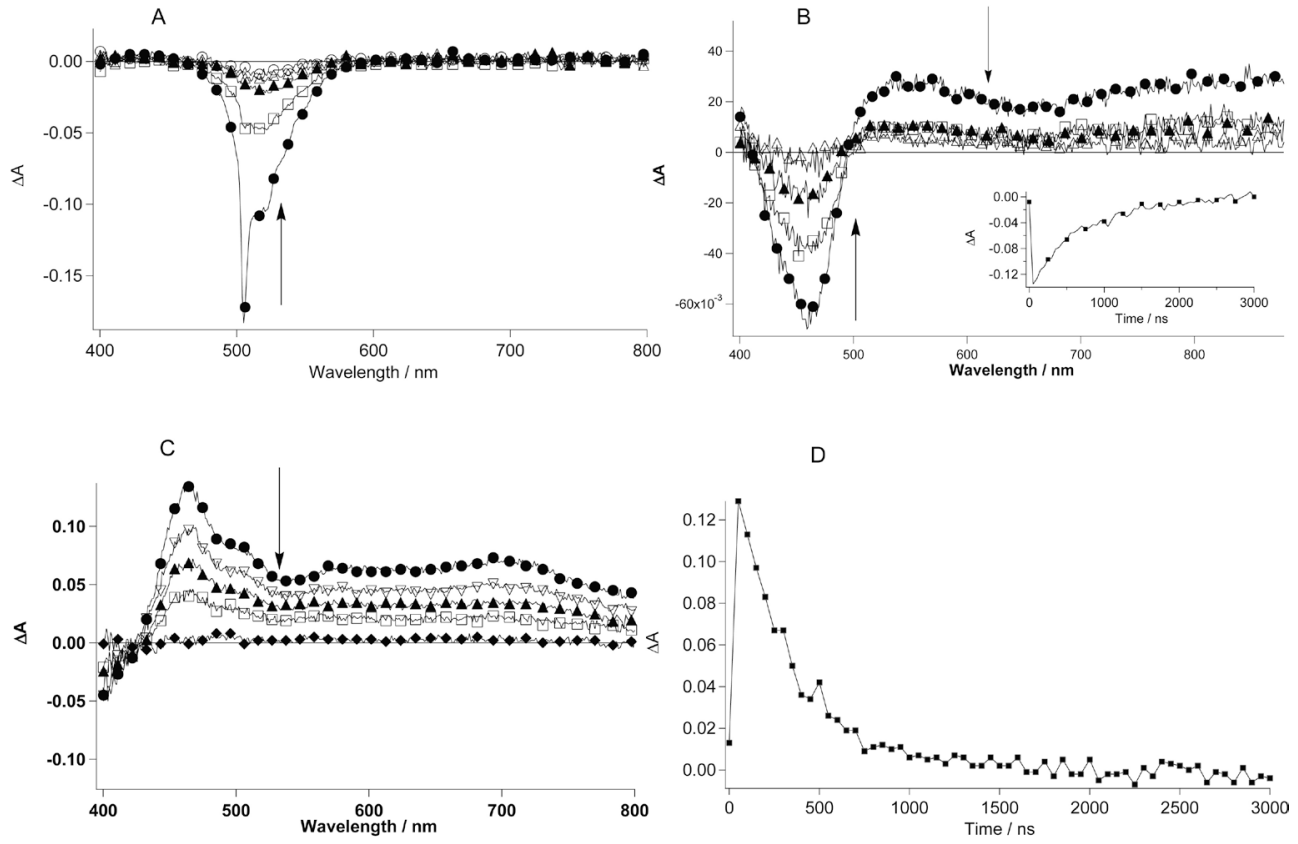

Fig. 5.9: Nanosecond transient absorption spectrum of (A) CdTe, (B) Ru-SAc with inset showing kinetics at $461 \mathrm{~nm}$ with lifetime $\tau=725 \mathrm{~ns}$ and (C) $\mathrm{CdTe} / \mathrm{Ru}-\mathrm{SAc}$ recorded at different times between 10 to $3200 \mathrm{~ns}$ after the laser pulse. $\lambda_{\text {ex }}=500 \mathrm{~nm}$.

(D) Kinetics monitored at $465 \mathrm{~nm}$ with lifetime $\tau=325 \mathrm{~ns}$.

This very slow process can be due to the very high exoergonicity of the back electron transfer, indicating that the process is in the inverted Marcus region. ${ }^{48-}$ 49 The full recovery of the spectra suggests that no decomposition occurred during the experiments. Even the control experiment shows a similar trend in the nanosecond transient absorption with comparable lifetime value as shown in Figure 5.10. 

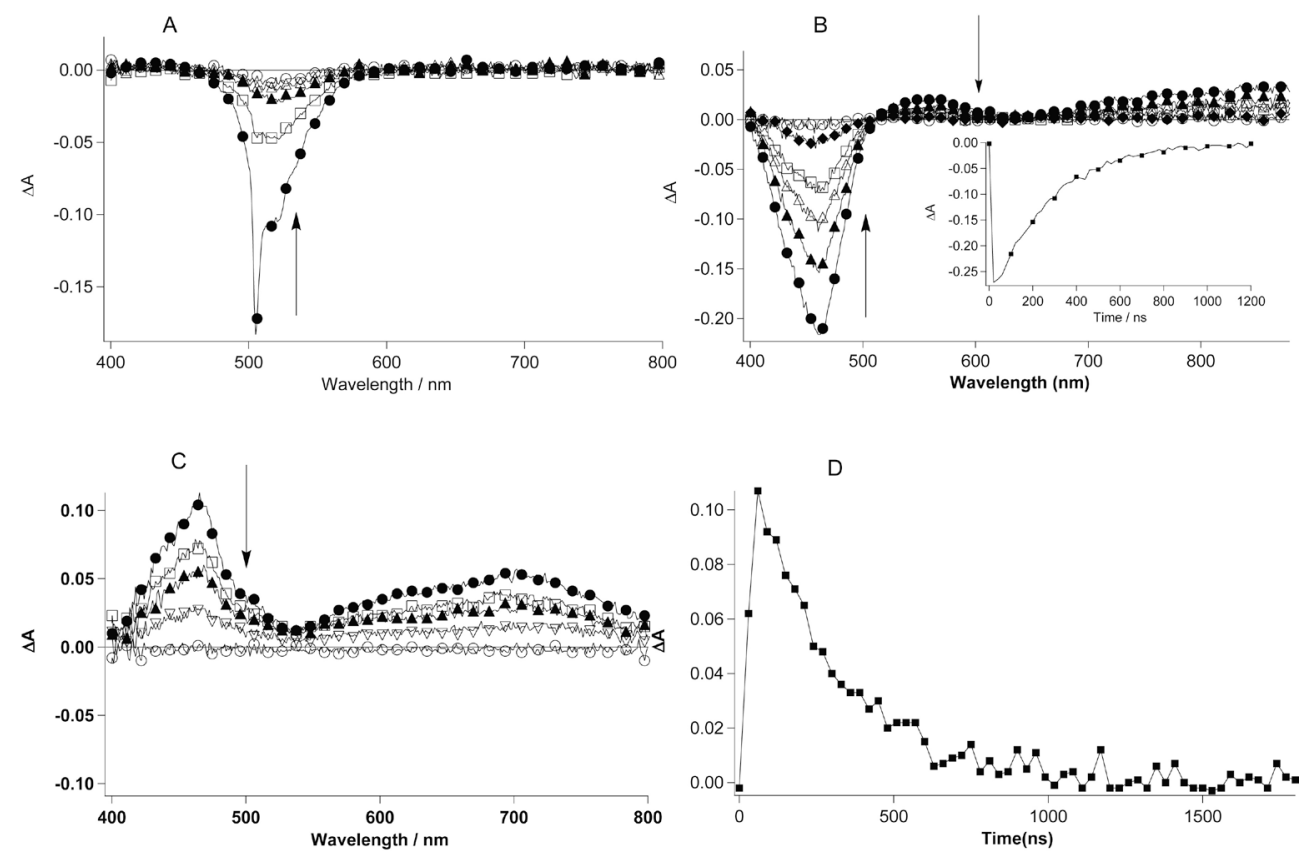

Fig. 5.10: Nanosecond transient absorption spectrum of (A) CdTe, (B) Ru-tert-Bu with inset showing kinetics at $461 \mathrm{~nm}$ with lifetime $\tau=266 \mathrm{~ns}$ in aerated condition and (C) CdTe-Ru-tert-Bu recorded at different times between 10 to $1200 \mathrm{~ns}$ after the laser pulse. $\lambda_{\text {ex }}=500 \mathrm{~nm}$. (D) Kinetics monitored at $463 \mathrm{~nm}$ with lifetime $\tau=284 \mathrm{~ns}$.

Based on our experimental observations and comparing our results with literature precedent, we propose the scheme shown in Figure 5.11 for the photoinduced electron transfer between CdTe quantum dots and the ruthenium tripod molecule. 


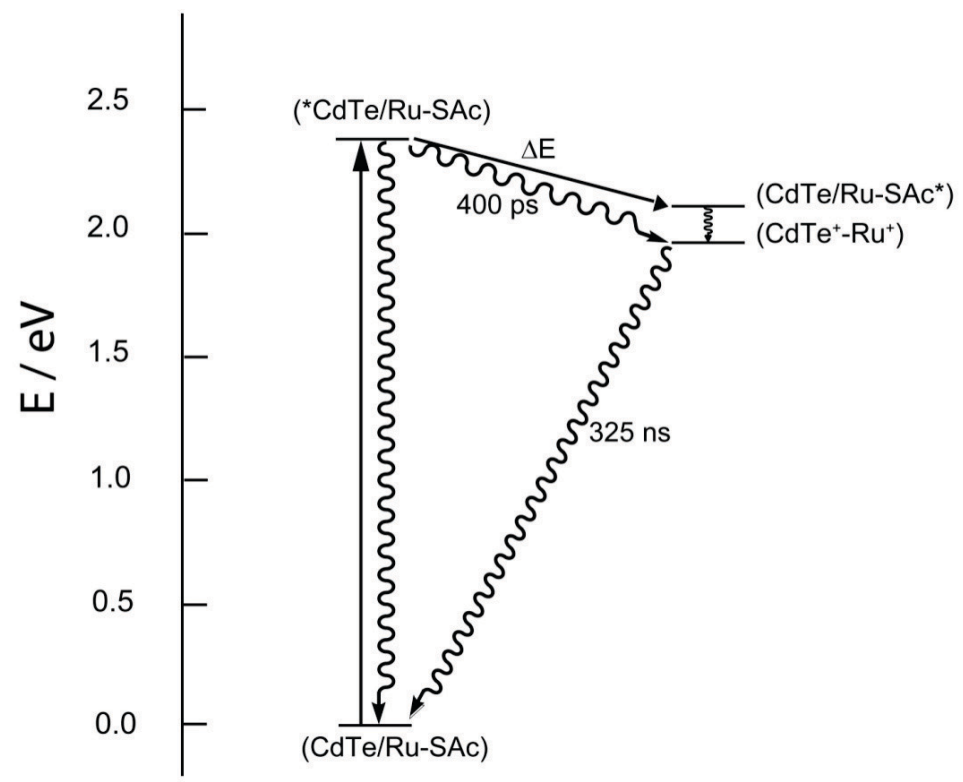

Fig. 5.11: Schematic representation of proposed energy level diagram for photoinduced process. The energy levels for the complexes are obtained from the electrochemistry. ${ }^{41}$ The straight arrow represents the energy transfer whereas the wavy arrow represents the (nonradiative) electron transfer process.

Upon excitation of quantum dots with $500 \mathrm{~nm}$ we can have two competitive processes, a very fast energy transfer to the excited state of ruthenium or an electron transfer from the QD to the ruthenium complex. As a result we can observe the formation of the charge separated state, $\mathrm{CdTe}^{+} \mathrm{Ru}^{+}$which decays in 325 ns back to the ground state.

\section{Conclusions}

In this chapter, the photophysical behavior of a quantum dot - metal complex based nanoassembly has been investigated through steady-state and timeresolved spectroscopic measurements. We report the results obtained using a tripod-based electroluminescent ruthenium complex with rigid legs and thioacetyl groups as terminal functionalities. A very fast energy transfer $(\sim 20$ ps) and a relatively slow electron transfer process $(\sim 400 \mathrm{ps})$ is observed in this assembly. Control experiments indicated that tripod molecules with thiol 
functionalization are bound to the quantum dot surface because of a shift in the absorption features, changes in transient features and faster electron transfer rate as compared to Ru-tert-Bu which has no anchoring groups. This study presents the possibility of employing the metal complex in combination with quantum dots for harvesting excitons, which is a key issue for photovoltaic applications. Further studies are warranted to identify ideal quantum dot and luminescent metal complex combinations to harness both energy and electron transfer processes effectively for optoelectronic applications.

\section{Experimental section}

General: Green-emitting CdTe nanocrystal quantum dots (NQD) capped with dodecylamine (DDA) and trioctylphoshphine (TOP) with a size of $3 \mathrm{~nm}$ were prepared according to the literature procedure. ${ }^{50} \mathrm{Ru}$-SAc and Ru-tert-Bu were prepared according to the literature procedure. ${ }^{41}$ All the spectroscopic solvents were purchased from Merck and used as received. Benzonitrile (99.9\%) was purchased from Aldrich. The solvents used for photophysical measurements were degassed with six subsequent freeze-pump-thaw cycles and then transferred to a glove box where samples were prepared.

\section{Determination of concentration of quantum dot solution}

The accurate determination of quantum dots in a solution is extremely difficult. However, there are some empirical methods available to determine the concentration based on absorption measurements for different nanocrystals. In this chapter, the concentration of quantum dots was estimated following the procedure reported by $\mathrm{Yu}$ and coworkers. ${ }^{51}$ Briefly, $20 \mu \mathrm{L}$ of quantum dots from the stock solution were taken and made up to $5 \mu \mathrm{L}$ by adding toluene. The absorption spectrum of this solution was measured. According to the empirical method published by Yu and coworkers, for CdTe nanocrystals, the size, $\mathrm{D}$ is given by

$$
\mathrm{D}=\left(9.8127 \times 10^{-7}\right) \lambda^{3}-\left(1.7147 \times 10^{-3}\right) \lambda^{2}+(1.0064) \lambda-194.84
$$


where $\lambda$ is the excitonic absorption maximum. Once the size is calculated, then the molar extinction coefficient is determined using the following expression.

$$
\varepsilon=10043(\mathrm{D})^{2.12}
$$

Upon knowing the epsilon value, it is possible to calculate the concentration of the quantum dots in the stock solution. It is noteworthy that the method reported above can underestimate the size of the nanocrystals. In such cases, it is also pragmatic to use the values obtained from high resolution transmission electron microscopy (HRTEM).

NQD/Ru complex: Dispersions of quantum dots and the ruthenium complexes were prepared inside the glove box: $250 \mu \mathrm{L}$ of CdTe quantum dots from the raw product $\left(4.2 \times 10^{-4} \mathrm{M}\right)$ were dispersed in $400 \mu \mathrm{L}$ toluene. The quantum dot concentration was determined using absorption spectroscopy according to the literature procedure. ${ }^{51}$ To this was added $3 \mathrm{~mL}$ of methanol to precipitate the quantum dots and the dispersion was subsequently centrifuged at $13200 \mathrm{rpm}$ for 20 minutes. The supernatant was discarded and the residue was dispersed in $10 \mathrm{~mL}$ of $1: 1$ toluene/benzonitrile to obtain $\sim 10^{-5}$ $\mathrm{M}$ quantum dot dispersion. A $10^{-4} \mathrm{M}$ stock solution of ruthenium tripodal molecules (Ru-SAc or Ru-tert-Bu) was prepared in the same solvent mixture. For all spectroscopic measurements (except transient absorption), the stock solutions were diluted tenfold. For transient absorption measurements, the stock solutions were used as prepared. In general, both Ru solutions and NQD dispersions were mixed in such a way that the ratio of $\mathrm{CdTe}$ to $\mathrm{Ru}$ tripod was 1:10 and stirred at room temperature for $\sim 1 \mathrm{~h}$ in order to ensure that the ruthenium complex would displace a sufficient number of capping ligands from the NQD surface. All the reference systems, Ru-SAc, Ru-tert-Bu, CdTe as well as the 1:10 mixture were prepared in such a way that the concentrations of the chromophores in reference and the complex dispersions were the same.

Photophysics: Absorption spectra were measured on a Varian Cary 5000 double-beam UV-Vis-NIR spectrometer and baseline corrected. Steady-state 
emission spectra were recorded on a HORIBA Jobin-Yvon IBH FL-322 Fluorolog 3 spectrometer equipped with a $450 \mathrm{~W}$ xenon arc lamp, double grating excitation and emission monochromators $(2.1 \mathrm{~nm} / \mathrm{mm}$ dispersion; 1200 grooves/mm) and a Hamamatsu R928 photomultiplier tube or a TBX-4$\mathrm{X}$ single-photon-counting detector. Emission spectra were corrected for source intensity (lamp and grating) and emission spectral response (detector and grating) by standard correction curves. Time-resolved measurements were performed using the time-correlated single-photon counting (TCSPC) option on the Fluorolog 3. NanoLED (402 nm; FWHM $<750$ ps) with repetition rates between $10 \mathrm{kHz}$ and $1 \mathrm{MHz}$ were used to excite the sample. The excitation sources were mounted directly on the sample chamber at $90^{\circ}$ to a double grating emission monochromator $(2.1 \mathrm{~nm} / \mathrm{mm}$ dispersion; 1200 grooves $/ \mathrm{mm}$ ) and collected by a TBX-4-X single-photon-counting detector. The photons collected at the detector are correlated by a time-to-amplitude converter (TAC) to the excitation pulse. Signals were collected using an IBH DataStation Hub photon counting module and data analysis was performed using the commercially available DAS6 software (HORIBA Jobin Yvon IBH). The quality of fit was assessed by minimizing the reduced chi squared function $\left(\chi^{2}\right)$ and visual inspection of the weighted residuals.

Transient absorption spectroscopy: For nanosecond transient absorption spectra, a sample with optical density of around 0.8 at the excitation wavelength $(500 \mathrm{~nm})$ was excited with a laser pulse from a MOPO pumped by a Q-switched beam locked Nd:YAG laser with a 10 ns pulse width and $10 \mathrm{~Hz}$ repetition rate. The excited state absorption was probed using a Xe flash lamp with the same repetition rate. The pulse energy was $2 \mathrm{~mJ} /$ pulse. The signal was recorded with a $\mathrm{CCD}$ camera as the detector. The excited state spectra were obtained by $\Delta \mathrm{A}=\log \left(\mathrm{I} / \mathrm{I}_{0}\right)$. The spectra were processed on an in house Lab View program.

For femtosecond transient absorption measurements, the laser system employed was based on a Spectra Physics Hurricane Ti-sapphire regenerative amplifier system. This optical bench assembly of the Hurricane included a seeding pump laser (Mai Tai), a pulse stretcher, a Ti-saphire regenerative amplifier, a Q-switched pump laser (Evolution) and a pulse compressor. The 
output power of the laser was typically $1 \mathrm{~mJ} /$ pulse (130 fs fwhm) at a repetition rate of $1 \mathrm{kHz}$. The pump-probe setup employed a full spectrum setup based on two optical parametric amplifiers (Spectra-Physics OPA 800) as a pump (depending on the excitation wavelength) and a residual fundamental light $(150 \mu \mathrm{J} /$ pulse $)$ from the pump OPA was used for the generation of white light, which was detected with CCD spectrograph. The OPA was used to generate excitation pulses from $280-600 \mathrm{~nm}$ (fourth harmonic signal of the OPA or idler). The white light generation was accomplished by focusing the fundamental $(800 \mathrm{~nm})$ into a stirred water cell equipped with barium bisfluoride or sapphire windows. The pump light was passed over a delay line (Physik Instrumente, M-531DD) that provided an experimental time window of $1.8 \mathrm{~ns}$ with the maximum resolution of $0.6 \mathrm{fs} / \mathrm{step}$. The energy of the probe pulses was $c a .5 \times 10^{-3} \mathrm{~mJ} /$ pusle. The angle between the pump and the probe beam was typically $7-10^{\circ}$. Samples were prepared in quartz cuvette $(1=0.1$ $\mathrm{cm})$ to have an optical density of $c a .0 .8$ at the excitation wavelength. For the white light/CCD setup, the probe beam was coupled into a $400 \mu \mathrm{m}$ optical fiber after passing through the sample, and detected by a CDD spectrometer (Ocean Optics, PC2000). The chopper (roffin Ltd., $f=10-20 \mathrm{~Hz}$ ), placed in the excitation beam, provided $\mathrm{I}$ and $\mathrm{I}_{0}$, depending on the status of the chopper (open or closed). The excited state spectra were obtained by $\Delta \mathrm{A}=\log \left(\mathrm{I} / \mathrm{I}_{0}\right)$. Typically, 2000 excitation pulses were averaged to obtain the transient at a particular time. Due to the lenses, a chirp of $c a .1$ ps is observed between $460-$ $650 \mathrm{~nm}$. 


\section{References}

1. Alivisatos, A. P., Science 1996, 271, 933.

2. Alivisatos, A. P., J. Phys. Chem. 1996, 100, 13226.

3. Nirmal, M.; Brus, L., Acc. Chem. Res. 1999, 32, 407.

4. Klimov, V. I., In Seimconductor and metal nanocrystals: Synthesis and Electronic Properties, Klimov, V. I., Ed. Marcel Deccer Inc.: New York, 2004.

5. Kongkanand, A.; Tvrdy, K.; Takechi, K.; Kuno, M.; Kamat, P. V., J. Am. Chem. Soc. 2008, 130, 4007.

6. Lopez-Luke, T.; Wolcott, A.; Xu, L. p.; Chen, S.; Wen, Z.; Li, J.; DeLaRosa, E.; Zhang, J. Z., J. Phys. Chem. C 2008, 112, 1282.

7. Leschkies, K. S.; Divakar, R.; Basu, J.; Enache-Pommer, E.; Boercker, J. E.; Carter, C. B.; Kortshagen, U. R.; Norris, D. J.; Aydil, E. S., Nano Lett. 2007, 7, 1793.

8. Gur, I.; Fromer, N. A.; Geier, M. L.; Alivisatos, A. P., Science 2005, 310, 462.

9. Colvin, V. L.; Schlamp, M. C.; Alivisatos, A. P., Nature 1994, 370, 354.

10. Munro, A. M.; Bardecker, J. A.; Liu, M. S.; Cheng, Y.-J.; Niu, Y.-H.; Plante, I. J.-L.; Jen, A. K. Y.; Ginger, D. S., Microchim. Acta 2008, 160, 345.

11. Tessler, N.; Medvedev, V.; Kazes, M.; Kan, S.; Banin, U., Science 2002, 295, 1506.

12. Daneshvar, H.; Nelms, J.; Muhammad, O.; Jackson, H.; Tkach, J.; Davros, W.; Peterson, T.; Vogelbaum, M. A.; Bruchez, M. P.; Toms, S. A., Nanomedicine 2008, 3, 21.

13. Hsieh, J.-M.; Ho, M.-L.; Wu, P.-W.; Chou, P.-T.; Tsai, T.-T.; Chi, Y., Chem. Commun. 2006, 615.

14. Klimov, V. I.; Mikhailovsky, A. A.; Xu, S.; Malko, A.; Hollingsworth, J. A.; Leatherdale, C. A.; Eisler, H. J.; Bawendi, M. G., Science 2000, 290, 314.

15. Wang, C.; Wehrenberg, B. L.; Woo, C. Y.; Guyot-Sionnest, P., J. Phys. Chem. B 2004, 108, 9027.

16. Callan, J.; De Silva, A.; Mulrooney, R.; Mc Caughan, B., J. Inclusion Phenom.Macrocyclic Chem. 2007, 58, 257. 
17. Nazzal, A. Y.; Qu, L.; Peng, X.; Xiao, M., Nano Lett. 2003, 3, 819.

18. Guldi, D. M.; Zilbermann, I.; Anderson, G.; Kotov, N. A.; Tagmatarchis, N.; Prato, M., J. Am. Chem. Soc. 2004, 126, 14340.

19. Guldi, D. M.; Zilbermann, I.; Anderson, G.; Kotov, N. A.; Tagmatarchis, N.; Prato, M., J. Mater. Chem. 2005, 15, 114.

20. Dayal, S.; Królicki, R.; Lou, Y.; Qiu, X.; Berlin, J. C.; Kenney, M. E.; Burda, C., Appl. Phys. B 2006, 84, 309.

21. Boulesbaa, A.; Issac, A.; Stockwell, D.; Huang, Z.; Huang, J.; Guo, J.; Lian, T., J. Am. Chem. Soc. 2007, 129, 15132.

22. Huang, J.; Stockwell, D.; Huang, Z.; Mohler, D. L.; Lian, T., J. Am. Chem. Soc. 2008, 130, 5632.

23. Campagna, S.; Puntoriero, F.; Nastasi, F.; Bergamini, G.; Balzani, V., Top. Curr. Chem. 2007, 280, 117.

24. Flamigni, L.; Barbieri, A.; Sabatini, C.; Ventura, B.; Barigelletti, F., Photochemistry and Photophysics of Coordination Compounds: Iridium. In Photochemistry and Photophysics of Coordination Compounds II, 2007; pp 143.

25. Kumaresan, D.; Shankar, K.; Vaidya, S.; Schmehl, R., Photochemistry and Photophysics of Coordination Compounds: Osmium. In Photochemistry and Photophysics of Coordination Compounds II, 2007; pp 101.

26. Sykora, M. P., M. A.; Alstrum-Acevedo, J.; Bezel, I.; Meyer, T. J.; Klimov, V. I.; J. Am.Chem. Soc. 2006, 128, 9984.

27. Schaller, R. D.; Sykora, M.; Jeong, S.; Klimov, V. I., J. Phys. Chem. B 2006, 110, 25332.

28. Anikeeva, P. O.; Madigan, C. F.; Coe-Sullivan, S. A.; Steckel, J. S.; Bawendi, M. G.; Bulovic, V., Chem. Phys. Lett. 2006, 424, 120.

29. Neuman, D.; Ostrowski, A. D.; Mikhailovsky, A. A.; Absalonson, R. O.; Strouse, G. F.; Ford, P. C., J. Am. Chem. Soc. 2008, 130, 168.

30. Medintz, I. L.; Farrell, D.; Susumu, K.; Trammell, S. A.; Deschamps, J. R.; Brunel, F. M.; Dawson, P. E.; Mattoussi, H., Anal. Chem. 2009, 81, 4831.

31. Jorge, P. A. S.; Mayeh, M.; Benrashid, R.; Caldas, P.; Santos, J. L.; Farahi, F., Appl. Opt. 2006, 45, 3760. 
32. Guo, W.; Yuan, J.; Li, B.; Du, Y.; Ying, E.; Wang, E., Analyst 2008, 133, 1209.

33. Jorge, P. A. S.; Maule, C.; Silva, A. J.; Benrashid, R.; Santos, J. L.; Farahi, F., Anal. Chim. Acta 2008, 606, 223.

34. Galoppini, E.; Guo, W.; Zhang, W.; Hoertz, P. G.; Qu, P.; Meyer, G. J., J. Am. Chem. Soc. 2002, 124, 7801.

35. Hoertz, P. G.; Carlisle, R. A.; Meyer, G. J.; Wang, D.; Piotrowiak, P.; Galoppini, E., Nano Lett. 2003, 3, 325.

36. Wuister, S. F.; de Mello Donega, C.; Meijerink, A., J. Phys. Chem. B. 2004, 108, 17393.

37. Guyot-Sionnest, P.; Wehrenberg, B.; Yu, D., J. Chem. Phys. 2005, 123, 074709.

38. Chestnoy, N.; Harris, T. D.; Hull, R.; Brus, L. E., J. Phys. Chem. 1986, 90, 3393 .

39. Sharma, S. N.; Pillai, Z. S.; Kamat, P. V., J. Phys. Chem. B 2003, 107, 10088.

40. Rehm, D.; Weller, A., Isr. J. Chem 1970, 8, 259.

41. Ramachandra, S.; Schuermann, K. C.; Edafe, F.; Belser, P.; Nijhuis, C. A.; Reus, W. F.; Whitesides, G. M.; De Cola, L., submitted.

42. Klimov, V. I.; McBranch, D. W.; Leatherdale, C. A.; Bawendi, M. G., Physical Review B 1999, 60, 13740.

43. Klimov, V. I., Semiconductor and Metal Nanocrystals. Synthesis and Electronic and Optical Properties. Marcel Dekker: New York, 2004; p 159

44. Kamat, P. V.; Dimitrijevic, N. M.; Nozik, A. J., The Journal of Physical Chemistry 2002, 93, 2873.

45. Klimov, V. I., Linear and nonlinear optical spectroscopy of semiconductor nanocrystals. In Handbook of Nanostructured Materials and Nanotechnology, Hari Singh, N.; M.Sc; Ph.D, Eds. Academic Press: Burlington, 2000; pp 451.

46. Guyot-Sionnest, P.; Shim, M.; Matranga, C.; Hines, M., Physical Review B 1999, 60, R2181.

47. Welter, S.; Salluce, N.; Benetti, A.; Rot, N.; Belser, P.; Sonar, P.; Grimsdale, A. C.; Mullen, K.; Lutz, M.; Spek, A. L.; DeCola, L., Inorg. Chem. 2005, 44, 4706. 
48. Closs, G. L.; Calcaterra, L. T.; Green, N. J.; Penfield, K. W.; Miller, J. R., J. Phys. Chem. 1986, 90, 3673.

49. Marcus, R. A., J. Chem. Phys. 1956, 24, 966.

50. Wuister, S. F.; Driel, F. v.; Meijerink, A., Phys. Chem. Chem. Phys. 5, 1253.

51. Yu, W. W.; Qu, L.; Guo, W.; Peng, X., Chem. Mater. 2003, 15, 2854. 
Photoinduced charge transfer between CdTe quantum dots and Ru tripod 


\section{CHAPTER 6}

\section{FRET IN QUANTUM DOT - DYE LOADED ZEOLITE - L ASSEMBLIES}

\section{ABSTRACT}

This chapter deals with nanoconjugates formed by functionalizing the dye loaded zeolite $\mathrm{L}$ surface with $\mathrm{CdSe} / \mathrm{ZnS}$ core-shell quantum dots. A photoinduced energy transfer is observed from quantum dots which are on the surface of the zeolite crystals to dye molecules encapsulated in the onedimensional channels of the aluminosilcate host. Two different dye molecules, thionine and oxonine, have been investigated in sensitization studies and it is demonstrated that energy transfer is most efficient with oxonine as acceptor. Further, steady-state and time resolved spectroscopy and microscopy are used to quantify the energy transfer occurring through dipole-dipole interactions (FRET) between CdSe/ZnS and oxonine loaded zeolite L crystals. 


\section{Introduction}

Host-guest chemistry which is a branch of supramolecular chemistry has been extensively studied for the last two decades. ${ }^{1-2}$ The weak interactions between the host and the guest molecules result in modulation of the guest species properties which would not be possible in their free state or in solution. A variety of host systems have been investigated such as proteins, ${ }^{3}$ cyclodextrins, ${ }^{4}$ dendrimers, ${ }^{5-6}$ polymers, ${ }^{7}$ glasses, ${ }^{8}$ or semiconductors ${ }^{9-10}$ to name a few. Amongst them, a very versatile host class is zeolites. ${ }^{11-13}$ These are microporous materials having a cage like structure made up of interlocked $\mathrm{SiO}_{2}$ and $\mathrm{Al}_{2} \mathrm{O}_{3}$ tetrahedra. Further, these microcrystalline materials possess long one, two or three dimensional channels which can encapsulate guest moieties. About 40 different types of zeolites are found in nature and more than 100 types have been synthesized. Out of these artificial zeolites, four have been widely studied for different applications, namely zeolite A, L, X and Y. ${ }^{14}$ The crystal size of zeolite $\mathrm{L}$ can be tuned from $30 \mathrm{~nm}$ to about 10 micron. In particular, from the optical/optoelectronic application point of view, zeolite L has been extensively employed as host. ${ }^{15-20}$ This crystalline material is an aluminosilcate with hexagonal symmetry and has a large number of one-dimensional channels running parallel to the crystals long axis with apertures of $7.1 \AA$ and inner diameter of $12 \AA$. Due to this structural feature, small organic molecules can be encapsulated in these materials. ${ }^{21}$ Since the structure consists of silica(IV) and alumina(III) tetrahedra, these materials possess a negatively charged framework and hence charge compensating cations are present inside the channels. Both cationic and neutral organic molecules can be loaded into the channels of these crystals. Cationic molecules are inserted by ion exchange in solution, ${ }^{22-28}$ neutral ones are loaded by gas phase adsorption. ${ }^{29-30}$

The physical, chemical and photophysical properties of the organic molecules inside the channels are strongly dependent on the host environment. To be specific, the host can contribute to thermal, mechanical and photochemical stabilization of the encapsulated organic molecules. In particular the increase in photophysical stability for organic dyes inside the hosts provides very 
interesting prospects. Various strategies have been employed in this regard like having a luminescent molecule outside the channels (stopcock principle) and observing photoinduced processes from outside to inside or vice versa. ${ }^{31-32}$ The idea behind using such chromophores is to create an artificial antenna system that can mimic the naturally occurring photosynthesis process wherein stopper molecules are used to inject into or extract excitonic energy from the crystals. ${ }^{15}$, ${ }^{29,33-35}$ In nature, the chlorophyll molecule which harvests sunlight is protected in a protein environment such that it does not form quenching aggregates and effectively transport the absorbed light to the photo center through Förster resonance energy transfer (FRET). In case of the host guest system mentioned above, the role of zeolite $\mathrm{L}$ channels is to perform a role similar to that of the protein pocket by preventing the formation of quenching aggregates by encapsulated organic dye molecules. In addition to this, the one-dimensional channel system allows for fast FRET transport of the excitation energy to the crystal ends / basal surface.

In order to employ such systems for practical applications, it is of prime importance to have a detailed understanding of photoinduced processes in such systems. In this regard, different organic and organometallic guests have been employed for the realization of supramolecular host guest antenna systems. ${ }^{36-37}$ For good donor acceptor systems, it is important to have a donor molecule with good absorption properties. In other words, the system should have a very good absorption cross section and high oscillator strength. For this purpose, quantum dots have been chosen as donors in the present study since they have very good oscillator strength and high absorption cross section. Furthermore, they absorb at all wavelengths below their exciton absorption which makes them strong contenders as light harvesting molecules for photovoltaic applications. ${ }^{38-40}$ In addition, these nanocrystals are highly luminescent, photostable and have tunable optical band gap which offer a unique advantage over other conventional organic or organometallic systems. ${ }^{41-}$ ${ }^{44}$ This chapter deals with nanoconjugates based on zeolite L loaded with dye molecules and $\mathrm{CdSe} / \mathrm{ZnS}$ quantum dots. In particular two different acceptor dyes have been used in the energy transfer studies, namely thionine and oxonine with core shell quantum dots functioning as energy donors. Most efficient energy transfer was observed with oxonine as the acceptor. A detailed 
study and quantification of Förster Resonance Energy Transfer (FRET) between $\mathrm{CdSe} / \mathrm{ZnS}$ quantum dots and thionine as well as oxonine loaded zeolite L crystals is reported.

\section{Zeolite $\mathrm{L}$ as host}

The rationale behind choosing zeolite L stems from the following aspects: they have a negatively charged framework which allows one to insert cationic dyes very easily through simple ion exchange. In addition, neutral dyes can also be inserted via gas phase adsorption. Most importantly, the synthetic procedures for preparing these materials are well established and provide a very good control of their size (between $30 \mathrm{~nm}$ to 10 microns) and morphology. These crystalline materials possess a hexagonal symmetry and all the relevant structural parameters are listed along with a 3 dimensional structural representations in Figure 6.1. As can be seen from 6.1(B), these materials can be considered to be composed of channels running parallel to the central axis of the cylinder. ${ }^{45}$

(A)

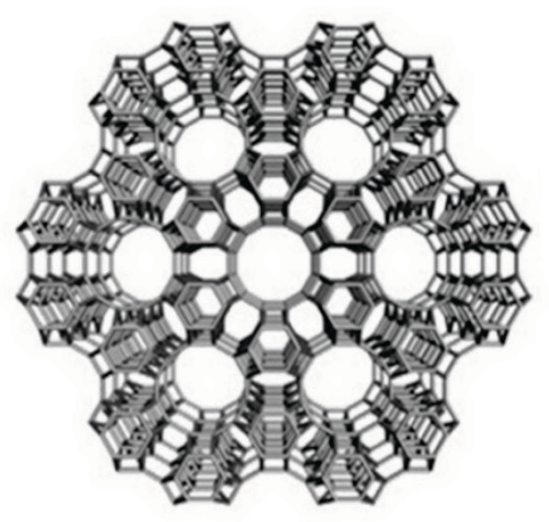

(B)

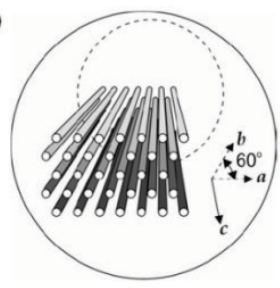

(D)

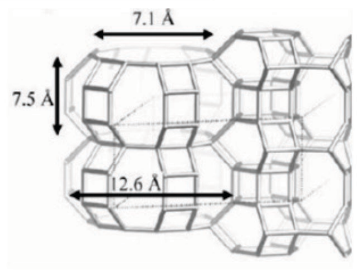

(C)

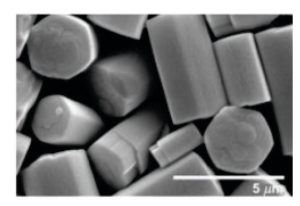

Figure 6.1: Schematic representation of zeolite L framework. (A) Projection along the $c$ axis of the crystal showing 7 channels (B) Schematic view of some channels with cylindrical morphology (C) SEM pictures of zeolite L crystals (D) Unit cell parameters of the crystals 
The number of such channels $n_{c b}$ can be calculated by approximating the zeolite $\mathrm{L}$ crystal as a cylinder with radius $r_{z}$ and is given by ${ }^{34}$

$$
n_{c h}=\frac{2 \pi}{\sqrt{3}}\left(\frac{r_{z}}{|\boldsymbol{a}|}\right)^{2}
$$

For zeolite L, the above equation can be simplified to

$$
n_{c h}=1.104 . r_{z}^{2}
$$

where $r_{z}$ is expressed in nanometers. Hence for a zeolite crystal with radius of about $500 \mathrm{~nm}$, there would be about 276000 parallel channels.

The guest molecules are situated at sites along the channels of the host species. A site is defined as the space occupied by one guest molecule and its length is usually expressed as the number $\mathrm{n}_{\mathrm{s}}$ times the unit cell length $\mathrm{c}$. A dye molecule which is about $15 \AA$ long would occupy two unit cells or $n_{s}=2$.

Due to the spatial restrictions imposed by the channels, usually only one organic dye molecule will occupy a site along these channels and aggregate formation can often be hindered.

Two different sizes of zeolite L crystals were used in this study: one with $1 \mu \mathrm{m}$ length $\mathrm{x} 1 \mu \mathrm{m}$ diameter for steady state emission measurements, and the other with dimensions of about 3.5 micron length $\mathrm{x} 1$ micron diameter for fluorescent lifetime imaging (FLIM) studies. Two different cationic dyes were inserted into these zeolite L crystals. Dye loading was in both cases carried out as ion exchange in water. For all measurements, the loading was maintained at about $5 \%$. The scanning electron microscopy (SEM) pictures of two different zeolite materials used in the chapter are shown in figure 6.2. 

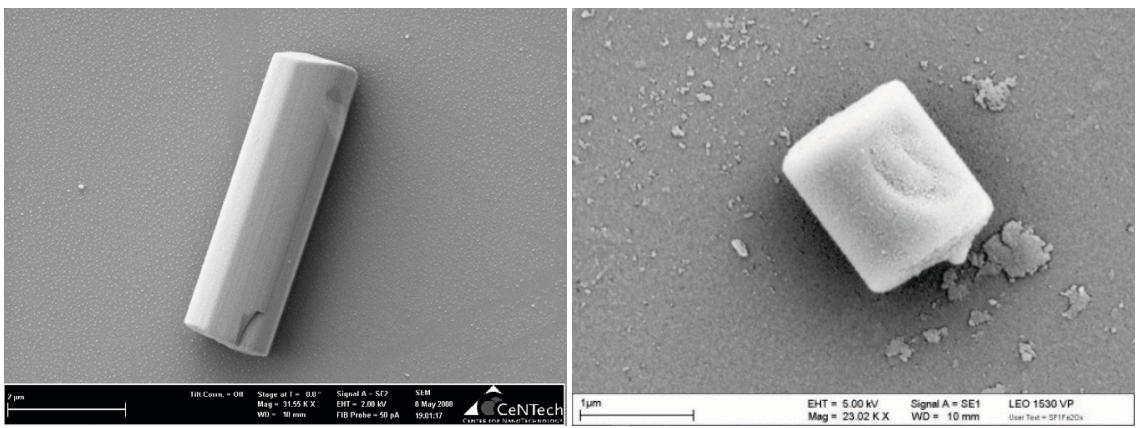

Figure 6.2: Scanning electron microscopy (SEM) images of long and the short zeolites used in this chapter. The long zeolites are between $3-4 \mu \mathrm{m}$ long and about $1 \mu \mathrm{m}$ wide and the short zeolites were $1 \mu \mathrm{m}$ long and $1 \mu \mathrm{m}$ wide.

\section{The guest dye molecules}

The two cationic dyes thionine and oxonine have been chosen for the present study. Both dyes have a similar structure with a molecular length of ca. $11.3 \AA$. These dye molecules were inserted by ion exchange in solution. The structural and photophysical properties of these dyes are reported in figure 6.3. 

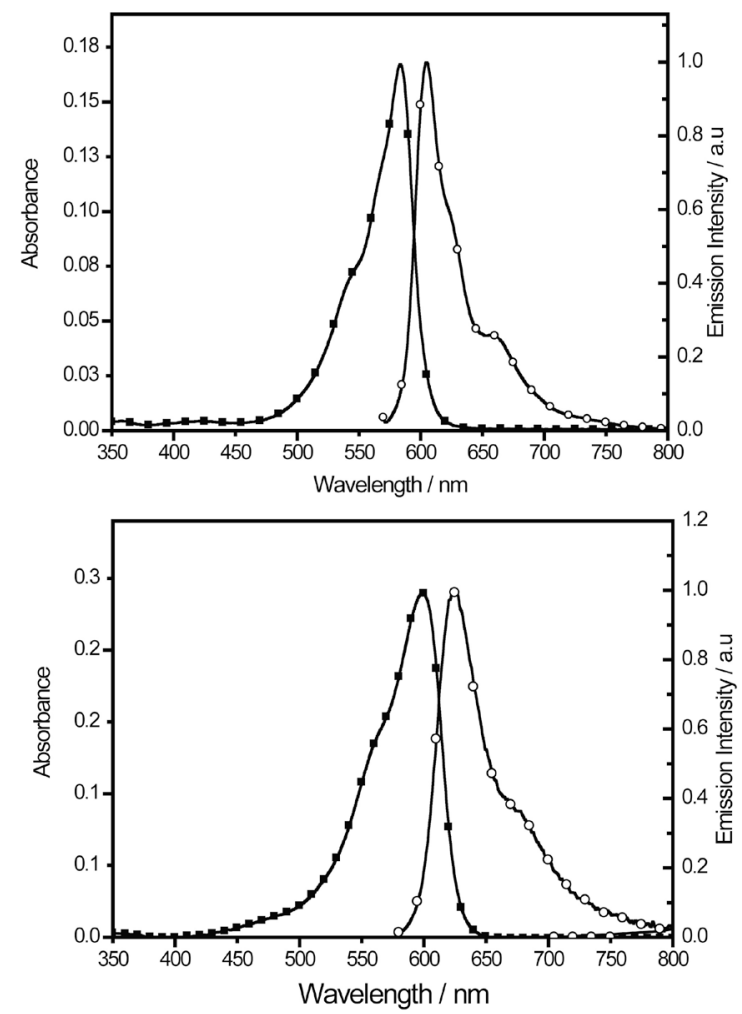<smiles>Nc1ccc2nc3ccc(=[NH2+])cc-3oc2c1</smiles>

\section{Oxonine}

Molecualr formula $=\mathrm{C}_{12} \mathrm{H}_{10} \mathrm{~N}_{3} \mathrm{O} \mathrm{Cl}$

Molecualr weight $=247.68 \mathrm{~g} / \mathrm{mol}$

$\varepsilon_{\mathrm{MeOH}}=84,100 \mathrm{dm}^{3} \cdot \mathrm{mol}^{-1} \cdot \mathrm{cm}^{-1}$

$\Phi_{\mathrm{MeOH}}=0.75$

$\tau_{\mathrm{MeOH}}=3.5 \mathrm{~ns}$<smiles>Nc1ccc2c(c1)CC1=CC(=[NH2+])C=CC1=[SH]2</smiles>

\section{Thionine}

Molecualr formula $=\mathrm{C}_{13} \mathrm{H}_{10} \mathrm{~N}_{2} \mathrm{SO} \mathrm{Cl}$

Molecualr weight $=265.55 \mathrm{~g} / \mathrm{mol}$

$\varepsilon_{\text {weter }}=53000 \mathrm{dm}^{3} \cdot \mathrm{mol}^{-1} \cdot \mathrm{cm}^{-1}$

$\Phi_{\text {water }}=0.10$

$\tau_{\text {water }}=0.360 \mathrm{~ns}$

Figure 6.2: Absorption and emission spectra of oxonine in methanol and thionine in water. Right panel shows the molecular structures and relevant photophysical parameters of the respective dye molecules.

\section{Interaction of quantum dots with zeolites}

The quantum dots used for the present study were core-shell CdSe/ZnS nanoparticles, which are commercially available (Evident Technologies, USA). Their relevant photophysical properties are presented in figure 6.3. The nanocrystals provided by the supplier are coated with trioctyl phosphine oxide (TOPO) and a matrix of aromatic hydrocarbons. The zeolites have hydroxyl groups on their surface. It was observed that these quantum dots have a very high affinity for the zeolite surface. Just by mixing the two solutions, we found 
that the nanocrystals stick all over the zeolite surface. The origin of this binding was initially thought to be electrostatic in nature based on the surface properties of both quantum dots and zeolites. However, once zeta potential measurements were carried out, it was found that both quantum dots and zeolite crystals have negative potentials. Thus any electrostatic interactions could be ruled out. At this point we can speculate that the interaction between quantum dots and zeolites may be due to van der Waals forces. More studies are warranted to corroborate this speculation.

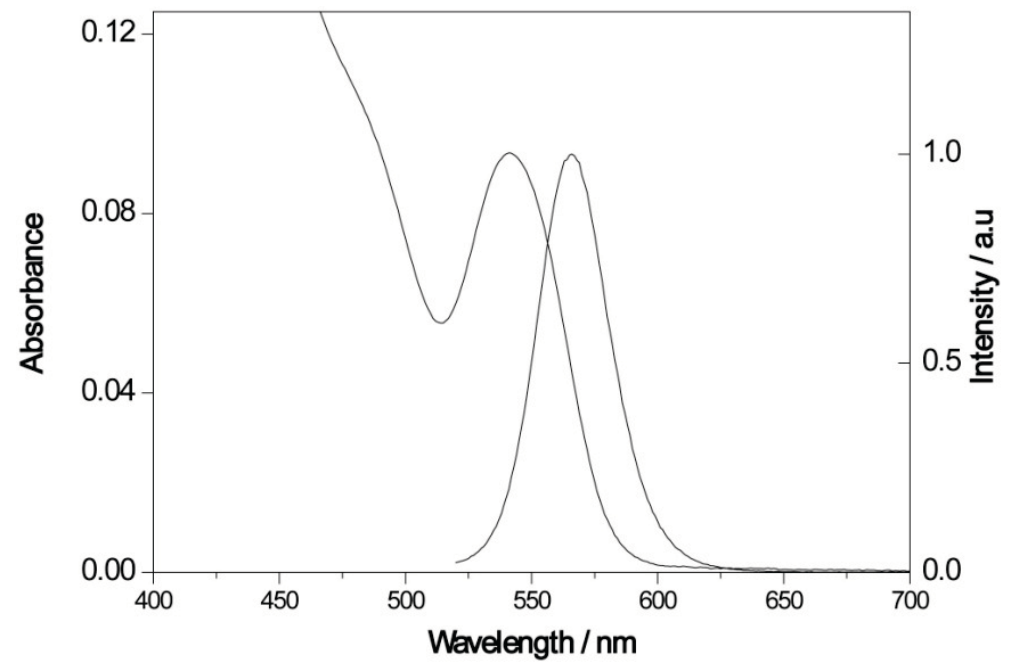

Figure 6.3: Absorption and emission spectra of CdSe/ZnS QDs in toluene. $\varepsilon_{545 \mathrm{~nm}}$ (in toluene) $=0.98 \times 10^{5} \mathrm{dm}^{3} \cdot \mathrm{mol}^{-1} \cdot \mathrm{cm}^{-1}$

The average amount of quantum dots taken up by zeolite crystals was estimated by absorption measurements. A known concentration of quantum dots in toluene was prepared and the absorption spectrum was measured. This solution was subsequently centrifuged at $3000 \mathrm{rpm}$ for 10 minutes and the absorption spectrum of the supernatant was measured. The spectra before and after centrifugation were identical, indicating that the centrifugation step does not precipitate any quantum dots. A known quantity of $1 \mu \mathrm{m}$ long empty zeolite crystals were added to this solution and the suspension was centrifuged for 10 minutes under identical conditions. The absorption spectrum of the supernatant (unbound quantum dots in toluene) was measured. The 
absorbance of the quantum dots was reduced significantly (figure 6.5) indicating that a lot of quantum dots were precipitated along with the zeolites. After the formation of nanoconjugate, the zeolites were washed repeatedly by centrifugation and the absorbance was found to be unaltered. In other words, quantum dots and zeolite crystals formed a stable conjugate. From the absorption difference, the amount of quantum dots before and after addition of zeolites was calculated. From this difference, the amount of quantum dots taken up for $1 \mu \mathrm{m} \times 1 \mu \mathrm{m}$ zeolites was found to be $14.5 \mathrm{pmol} / \mathrm{mg}$.

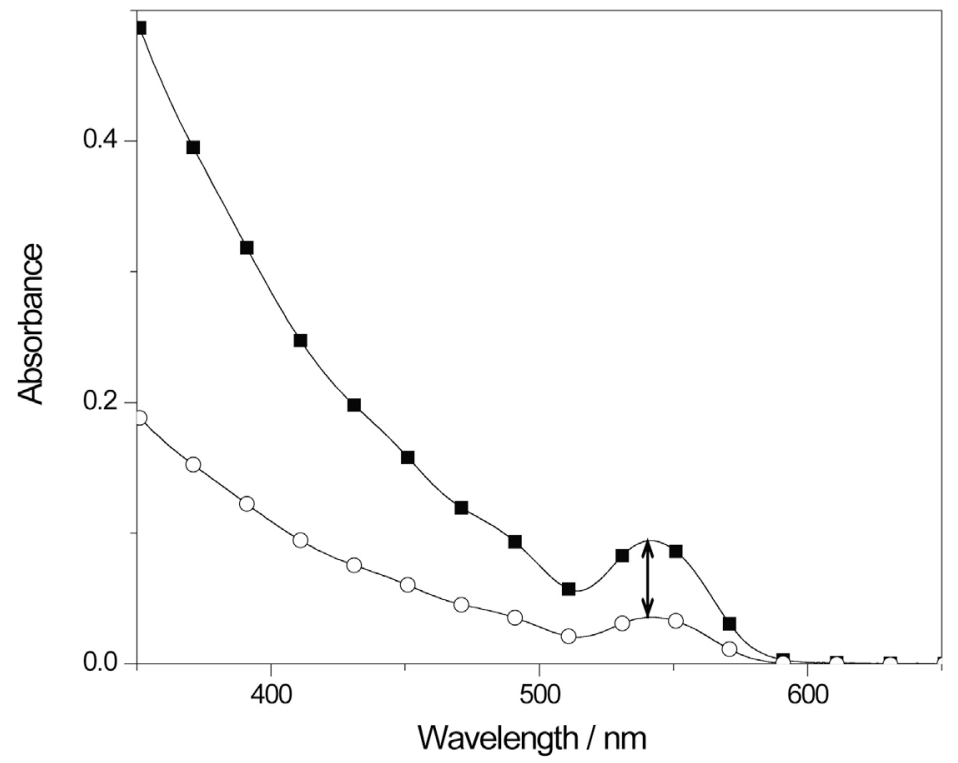

Figure 6.4: Absorption spectra of CdSe/ZnS QDs before centrifugation (--), after centrifugation (-०-) and after addition of zeolites and centrifugation $\left(-\diamond_{-}\right)$

The fluorescence microscopy images show clearly that the quantum dots stick to the whole zeolite surface (figure 6.5). The excited state lifetime of quantum dots was unaltered after being taken up by the zeolites; ruling out any quenching mechanism due to interaction with zeolites (see energy transfer studies for details). The size of the quantum dots is about $4 \mathrm{~nm}$ excluding the 
$\mathrm{ZnS}$ shell and the capping ligands and hence they are too large to enter the zeolite channels.

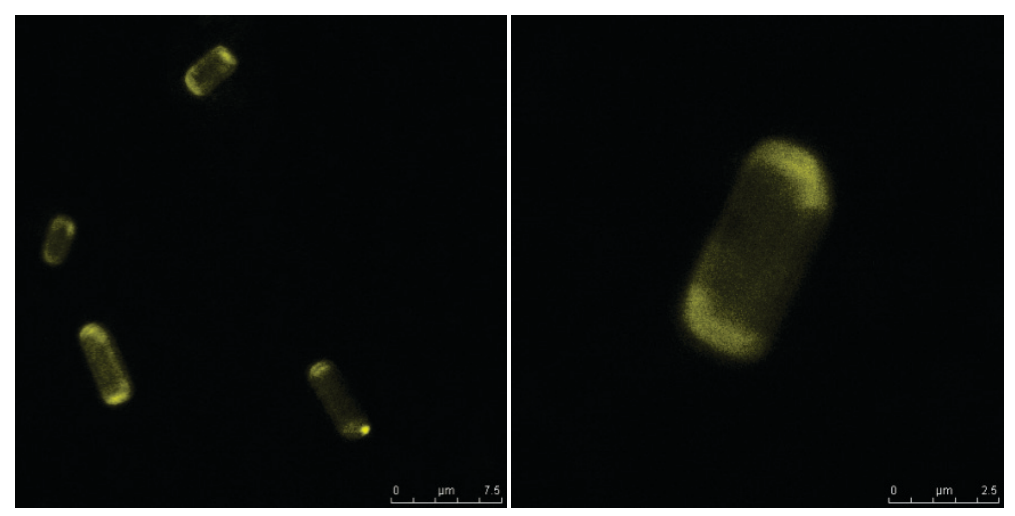

Figure 6.5: Florescence microscope images of zeolites coated with $\mathrm{CdSe} / \mathrm{ZnS}$ quantum dots.

\section{Energy transfer studies}

For dye loaded zeolites, it is evident that highest dye concentrations can be found at the channel entrances if the loading time is less than ca. 160 hours for dyes like pyronine and oxonine. In order to estimate the amount of quantum dots to be used per gram of zeolite crystals, the number of channel entrances was calculated as this gives the idea about the number of potential acceptor dyes for a given loading. The number of channel entrances (in moles) for zeolite L crystals was calculated assuming cylindrical geometry using the following equation ${ }^{46}$

$$
n_{e}=\frac{X_{Z}}{L_{Z}} 5.21 \cdot 10^{-7}
$$

where $X_{Z}$ is the mass of the zeolite sample in $\mathrm{mg}$ and $L_{Z}$ is the average length of the zeolite in $\mathrm{nm}$. For $1 \mathrm{mg}$ of $1 \mu \mathrm{m}$ long zeolites, the number of channel entrances was estimated to be $5.21 \times 10^{-10}$ moles. Considering the size of a 
quantum dot and the channel entrance spacing in zeolite L, we can approximate that about one quantum dot would occupy four channel entrances. Hence number of quantum dots required to fill up all the available sites turns out to be 130.25 pmoles. However, this is only an ideal case as it was found out that the quantum dots attach all over to the zeolite crystals (figure 6.5). Also for the quantum dots, the size of the $\mathrm{ZnS}$ shell and the capping ligands would further increase the size to about 6 to $8 \mathrm{~nm}$ and hence the above number turns out to be an overestimation. This is also evident by the uptake experiment which resulted in about 14.5 pmoles per mg of $1 \mu \mathrm{m}$ long zeolite L crystals. Hence for the photoluminescence studies using about $3.5 \mathrm{~mL}$ of an oxonine loaded zeolite $\mathrm{L}$ suspension, 52 pmoles of quantum dots were used. The reason behind using a slight excess of quantum dots stems from the fact that their concentration is determined from an empirical method based on absorption measurements ${ }^{47}$ which underestimates the size of the nanocrystals.

\section{CdSe/ZnS - thionine loaded zeolite L}

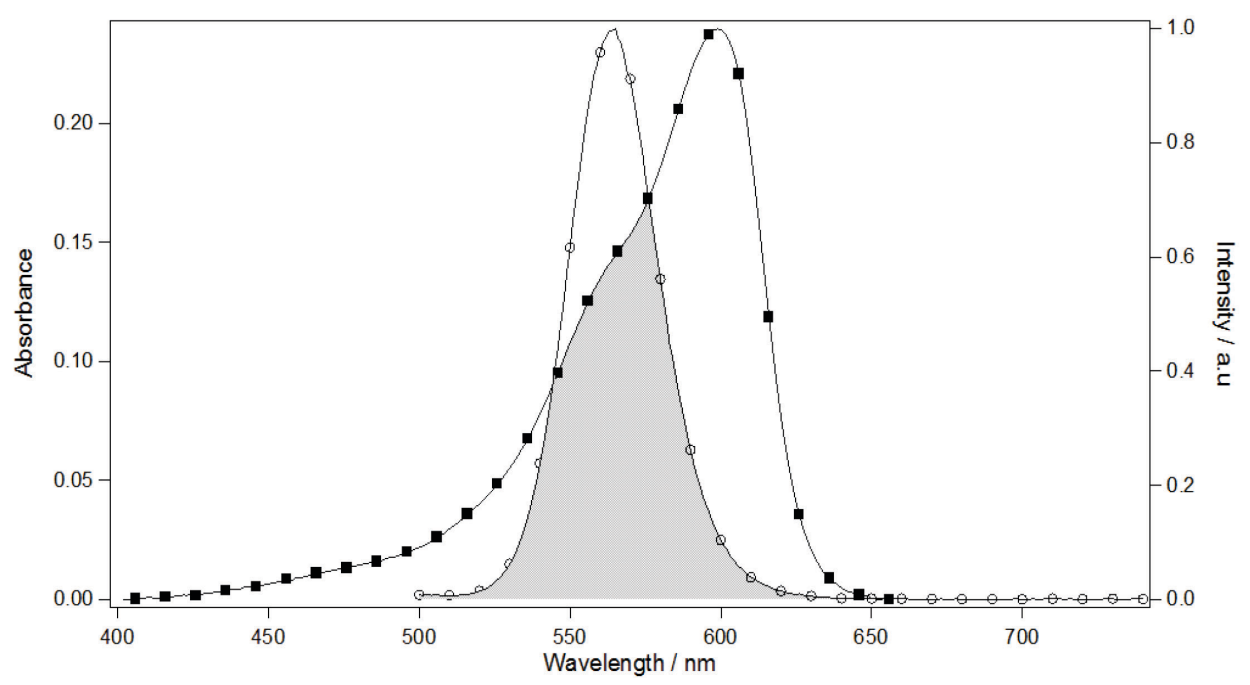

Figure 6.6: Spectral overlap between $\mathrm{CdSe} / \mathrm{ZnS}$ emission (-o-) and the thionine absorption (--)

For the quantum dots and thionine loaded zeolites, the spectral overlap is decent as indicated by the shaded area in the spectra of figure $6.6,1 \mu \mathrm{m} \times 1 \mu \mathrm{m}$ 
long zeolites were used and the thionine dye was loaded through ion exchange as described in the experimental section. The stock solution of quantum dots was prepared in such a way that $1 \mu \mathrm{l}$ corresponds to 52 pmoles. Upon addition of $1 \mu \mathrm{l}$, the volume change in the zeolite suspension $(3.5 \mathrm{ml})$ is negligible. The steady-state emission measurements were carried out by exciting the samples at $400 \mathrm{~nm}$ as the thionine absorbs very little light at this wavelength. The dye loading was $5 \%$ and the zeolites were suspended in toluene and stirred continuously during measurements. After measuring the emission of zeolites loaded with thionine, $1 \mu \mathrm{l}$ (52 pmoles) of quantum dot dispersed in toluene was added to the sample. The mixture was stirred for ten minutes and the emission spectrum was recorded again. As can be seen from figure 6.7, addition of quantum dots resulted in the appearance of a band at $565 \mathrm{~nm}$, which corresponds to the exciton emission of the quantum dots and in an intensity increase of the band at $640 \mathrm{~nm}$, corresponding to the emission of thionine.

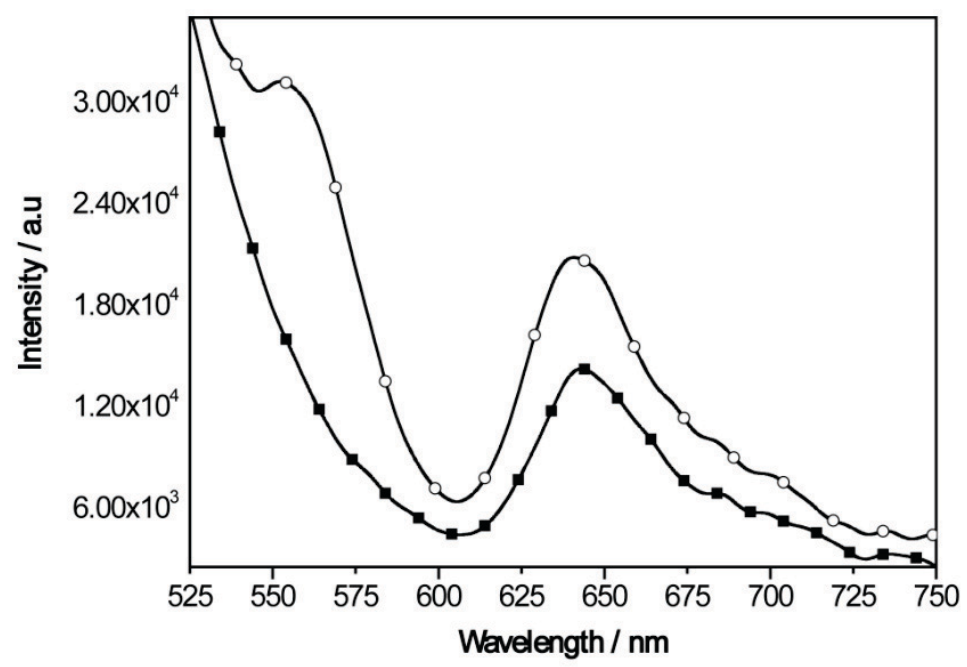

Figure 6.7: Emission spectra of zeolite loaded with thionine in absence of any quantum dots (--) and after adding $1 \mu \mathrm{l}(52 \mathrm{pmol})$ of $\mathrm{CdSe} / \mathrm{ZnS}$ quantum dots in toluene (-o-). $\lambda_{\mathrm{em}}=400 \mathrm{~nm}$

To further eliminate any contribution from the high background signal - arising from zeolite scattering - and the emission tail from the quantum dots, a 
control experiment was performed with unloaded zeolite under identical experimental conditions. As can be seen from the emission spectra of the control experiment (figure 6.8), there is no emission band at 640 showing that the previously observed (figure 6.7) belongs to thionine.

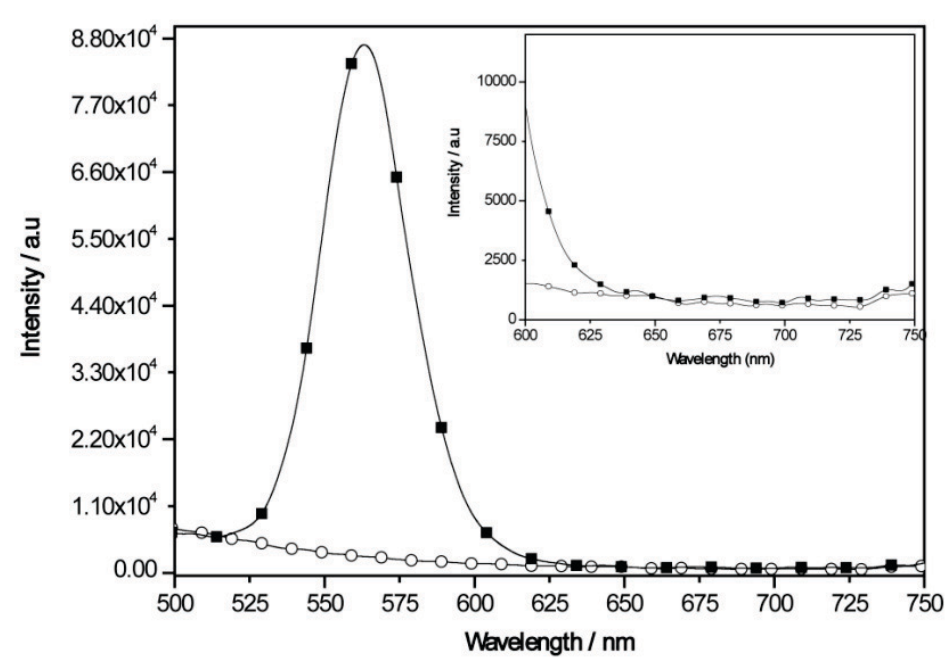

Figure 6.8: Emission of empty zeolite $\mathrm{L}$ in absence of any quantum dots (-o-) and after adding $1 \mu \mathrm{l}(52 \mathrm{pmol})$ of $\mathrm{CdSe} / \mathrm{ZnS}$ quantum dots in toluene (--). $\lambda_{\mathrm{em}}=400 \mathrm{~nm}$

From the above experiments, one can conclude that the intensity increase of thionine emission is indeed due to sensitization. The excited state lifetimes were measured using a time correlated single photon counting (TCSPC) system. Quantum dots adsorbed on empty zeolite surfaces yielded a triexponential decay with lifetimes of $4.6 \mathrm{~ns}(12 \%), 15 \mathrm{~ns}(76 \%)$ and $43 \mathrm{~ns}(12 \%)$. The 15 ns component can be attributed to exciton recombination in the nanocrystals whereas the shorter and much longer components (with weights around $12 \%$ each) could be attributed to the size inhomogenities and trap state emission. However, to simplify the analysis, the average lifetime $\langle\tau\rangle$ is a good approximation. From the present set of data, $\langle\tau\rangle$ for quantum dots was found 
to be 17.1 ns. Inside the zeolite channels, thionine exhibits a biexponential lifetime with components of $0.55 \mathrm{~ns}(24 \%)$ and $3.8 \mathrm{~ns}(76 \%)$. The $0.55 \mathrm{~ns}$ component is very close to the excited state lifetime of thionine in solution and hence the origin of this component could be due to some thionine molecules adsorbed on the outer surface. However, $\langle\tau\rangle$ was calculated to be $3.02 \mathrm{~ns}$. In the nanoconjugate, the lifetime of the quantum dot exciton recombination of was quenched to $9.3 \mathrm{~ns}$, whereas the shorter component was quenched to 2.7 ns and longer component was reduced to 25 ns. The average lifetime was found to be 12.5 ns. From the above results, we find that, despite a good spectral overlap, the energy transfer in the above donor-acceptor system turned out to be not very efficient $(30 \%)$.

\section{CdSe/ZnS - Oxonine loaded zeolite L}

Oxonine was chosen for the present study with quantum dots because of its high quantum yield and absorption coefficient as well as good spectral overlap with the yellow emitting quantum dots as can be seen from the dashed area in figure 6.9.

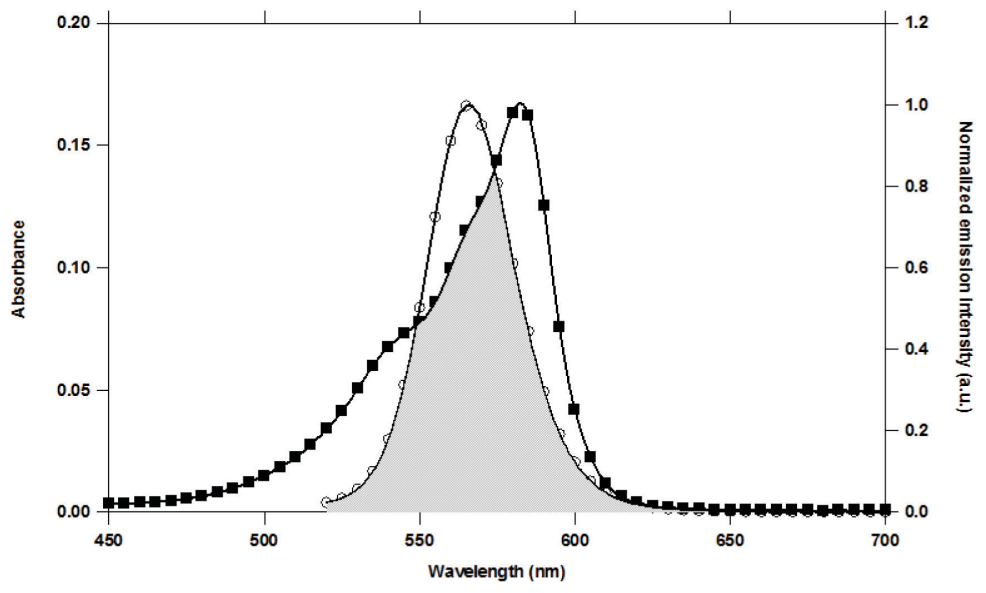

Figure 6.9: Spectral overlap between quantum dot emission (-o-) and the oxonine absorption (--) 
For these experiments, the same two zeolite L materials were used. $1 \mu \mathrm{m}$ long zeolites were used for measuring the photoluminescence and lifetimes were recorded with $3.5 \mu \mathrm{m}$ long zeolites by means of fluorescence lifetime imaging (FLIM). In both cases, $1 \mu \mathrm{l}$ of quantum dot stock solution, corresponding to 52 pmoles, was added. For photoluminescence measurements, addition of $1 \mu \mathrm{l}$ to the total volume of $3.5 \mathrm{ml}$ results in a negligible volume change. The concentration of the zeolite $\mathrm{L}$ suspension was $1 \mathrm{mg} / \mathrm{ml}$ in toluene with $5 \%$ dye loading. During measurements, the suspensions were stirred continuously to avoid sedimentation. The emission spectrum of zeolites loaded with only oxonine was measured. $1 \mu \mathrm{l}$ of quantum dot stock solution, corresponding to 52 pmoles, was added afterwards and the suspension was stirred for 10 minutes before recording the spectrum again. As can be seen from the spectra in figure 6.10, addition of quantum dots sensitizes the oxonine emission by a factor of about three. Upon further addition of quantum dots the intensity of oxonine emission did not change indicating that all available channel entrances were successfully occupied by nanocrystals. The possibility of FRET is limited to the channel entrances due to high dye concentrations found there. This is, of course, under the assumption that no dye molecules are adsorbed on the outer surface and hence no energy transfer is possible from QDs to these dyes adsorbed on the surface. 


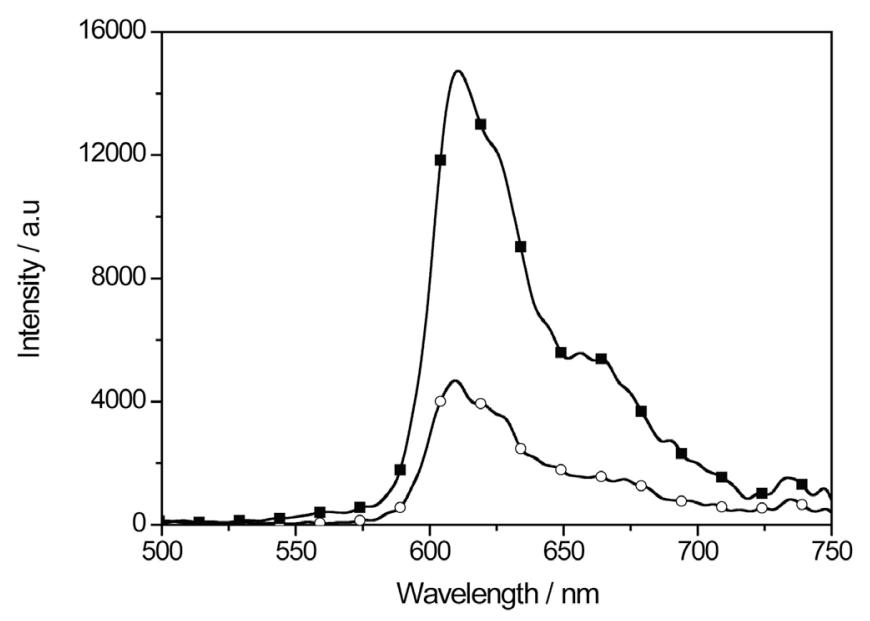

Figure 6.10: Emission of zeolite loaded with oxonine in absence of any quantum dots $\left(-{ }^{-}\right)$and after adding $1 \mu \mathrm{l}(52 \mathrm{pmol})$ of $\mathrm{CdSe} / \mathrm{ZnS}$ quantum dots in toluene (--). $\lambda_{\mathrm{em}}=$ $386 \mathrm{~nm}$

In order to rule out any effects from the host material, control experiments with empty zeolite L crystals were performed under identical conditions. From the spectra shown in figure 6.11, it is evident that the empty zeolite host material does not emit light in the emission range of oxonine. It is clear that increase in the emission intensity at $610 \mathrm{~nm}$ observed in figure 6.10 is indeed due to sensitization. 


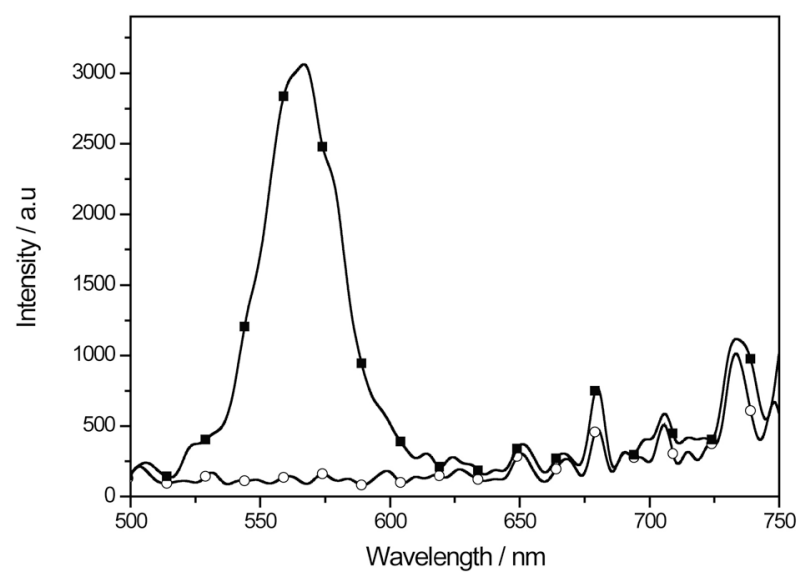

Figure 6.11: Emission of empty zeolite $\mathrm{L}$ in absence of any quantum dots (-०-) and after adding $1 \mu \mathrm{l}(52 \mathrm{pmol})$ of $\mathrm{CdSe} / \mathrm{ZnS}$ quantum dots in toluene (--). $\lambda_{\mathrm{em}}=386 \mathrm{~nm}$

In order to measure the excited state lifetime of quantum dots, fluorescence lifetime imaging was carried out on a time resolved confocal microscope. For this study, longer zeolites of about $3.5 \mu \mathrm{m}$ length loaded with oxonine were employed. $1 \mu \mathrm{l}$ of quantum dot stock solution were added to a suspension of these dye loaded zeolites in toluene and the mixture was stirred for ten minutes. The nanoconjugates were washed by first centrifuging at $1000 \mathrm{rpm}$ for ten minutes and discarding the supernatant to get rid of any unbound quantum dots. The sedimented crystals were redispersed in toluene. This washing procedure was repeated three times to ensure that unbound quantum dots were completely washed off. Confocal microscopy images of these crystals were recorded using a $440 \mathrm{~nm}$ pulsed laser as excitation source. A beam splitter was used to separate the emission from quantum dots and oxonine into two detector channels. The fluorescence microscope images of quantum dots with empty zeolites and quantum dots with dye loaded zeolites are shown in figure 6.12 . 


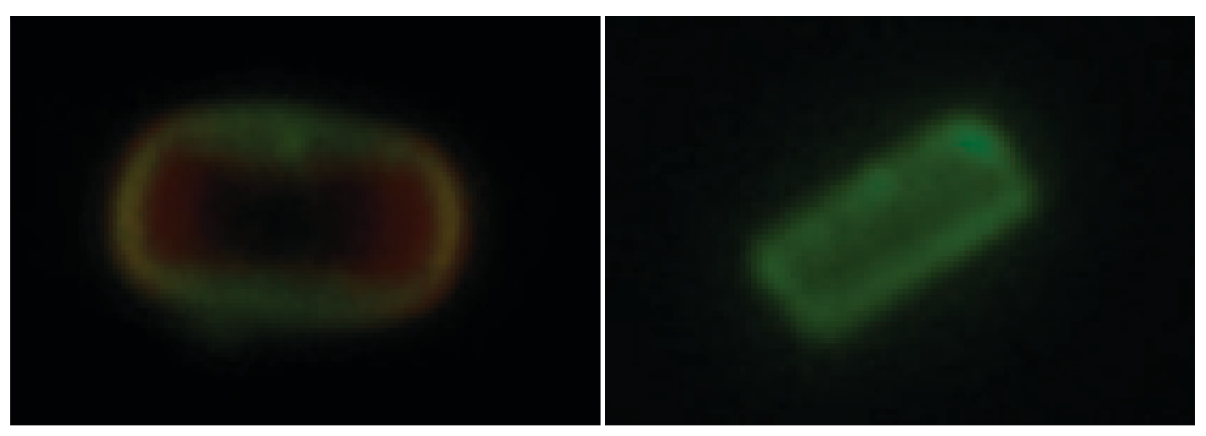

Figure 6.12: Florescence microscope images of zeolites loaded with oxonine and coated with CdSe/ZnS quantum dots (left) as well as empty zeolites coated with $\mathrm{CdSe} / \mathrm{ZnS}$ quantum dots.

As can be seen from the left panel of figure 6.12, the dye molecules are mostly concentrated at the channel entrances, while the quantum dots bind all over the surface. The excited state lifetimes were measured using a time correlated single photon counting (TCSPC) system. The quantum dots adsorbed on the zeolite L samples yielded a biexponential decay of $7.86 \mathrm{~ns}(45 \%)$ and $24 \mathrm{~ns}$ $(55 \%)$. The average lifetime $\langle\tau\rangle$ for the quantum dots adsorbed onto the zeolite surface was calculated to be 16.08 ns. For quantum dots coated on the surface of zeolites loaded with oxonine, however, the lifetimes were much lower. It was found that the components of the quenched lifetimes were 2.16 ns $(80 \%)$ and $13.54 \mathrm{~ns}(20 \%)$ and hence $\langle\tau>$ was calculated to be $4.4 \mathrm{~ns}$. It is evident that both sensitization of oxonine emission as well as quenching of quantum dot lifetimes are very efficient in this system. 


\section{Quantification of FRET}

Photoinduced energy transfer can occur through either electron exchange (Dexter type) or through dipole-dipole interaction (Förster mechanism). For the Dexter mechanism, the prerequisite is orbital overlap between donor and acceptor molecules. ${ }^{48}$ For this to happen, both species should be in extremely close proximity $(\leq 1 \mathrm{~nm}$ ). In case of zeolites loaded with dye molecules, the dyes will first be located close to the channel entrances and can diffuse up to about $100 \mathrm{~nm}$ depending on the insertion time. The quantum dots have a $\mathrm{ZnS}$ shell and capping ligands which would increase the distance between the donor and the acceptor. Also quantum dots are too big to pass the zeolite pore opening $(0.71 \mathrm{~nm})$. Hence it is reasonably safe to exclude the Dexter mechanism in this case.

The conditions necessary for efficient energy transfer to be through Förster mechanism ${ }^{49-51}$ are (i) a good spectral overlap between the donor and acceptor, (ii) large radiative rate constant for the donor, (iii) large molar extinction coefficient of the acceptor and (iv) small separation between donor and acceptor as well as an appropriate relative orientation between their electronic transition dipole moments. In case presented here, assuming a spherical shape for the quantum dots and thereby an isotropic distribution of its transition dipole moment, all of the conditions mentioned above are satisfied. Firstly from figure 6.6 and 6.9, it is evident that in both the cases, the spectral overlap is good. The radiative lifetimes of quantum dots calculated from their excited state lifetimes and are about $10^{7}$ which are quite high. Both organic dye molecules have very high molar extinction coefficients of the order of about $10^{5}$. Since the quantum dots are adsorbed onto the surface of the zeolites, and the dye molecules are present at the channel entrances, there is a high probability of finding a large number of donor and acceptors at relatively close proximity, facilitating energy transfer. Hence, in both system used in this chapter, we can conclude that energy transfer occurs via a dipole-dipole interaction (Förster mechanism). A detailed description of FRET can be found in the introductory chapter of this thesis. The spectral overlap and the Förster radius were calculated using equations 1.2 and 1.3 (chapter 1) respectively. For the above systems, a MATCAD program obtained from Prof. Gion Calzaferri 
has been used to calculate the abovementioned parameters. The donoracceptor separation, $r$, can be estimated from the dependence of energy transfer efficiency on donor-acceptor distance and the Förster radius as shown by the sigmoidal curve depicted in figure 6.13

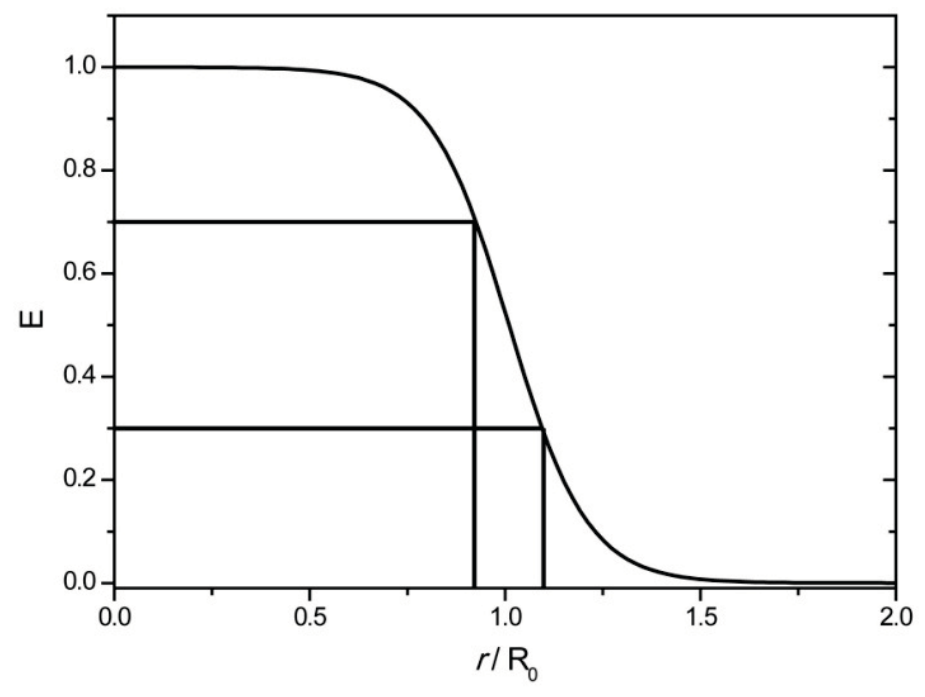

Figure 6.13: Dependence of energy transfer efficiency, $\mathrm{E}$ on distance. $\mathrm{R}_{0}$ is the Förster radius. ${ }^{52}$

The energy transfer efficiency was calculated using equation 3.1 (chapter 3) by employing the quenched and unquenched lifetimes of the donor. In case of zeolites loaded with thionine, the efficiency was found to be about $30 \%$. Using this value in the curve above, the $r / R_{0}$ value can be extracted and turns out to be 1.1 times $\mathrm{R}_{0}$. Hence the donor-acceptor separation was estimated to be $55.6 \AA$. By using the known parameters in equation 6.4, the energy transfer rate for this system was calculated to be $3.3 \times 10^{7} \mathrm{~s}^{-1}$. In case of oxonine loaded zeolites, the energy transfer efficiency was much higher. From the extent of quantum dot lifetime quenching, the efficiency was estimated to be about $70 \%$ and, in turn, the $r / R_{0}$ value was found out to be 0.92 , leading to a donoracceptor separation of $67.25 \AA$. From these parameters, the energy transfer rate was estimated to be $6.75 \times 10^{7} \mathrm{~s}^{-1}$. 


\section{Conclusions}

In this chapter a new class of assemblies is described based on microporous zeolite L crystals and semiconducting nanocrystals has been explored. Incorporating appropriate cationic dye molecules inside the porous framework confers luminescent properties to an otherwise optically transparent host material. Adsorbing the nanocrystals on the zeolite surface gives a system with energy donors outside and acceptors inside the channels. Two different cationic dyes, thionine and oxonine were incorporated into zeolite L crystals through ion exchange from water. In combination with $\mathrm{CdSe} / \mathrm{ZnS}$ quantum dots, Förster Resonance Energy Transfer (FRET) has been demonstrated and quantified using steady-state as well as time resolved spectroscopy and microscopy techniques. The energy transfer was found to be more efficient in case of oxonine - with 70\% efficiency. Such a vectorial energy transfer in host guest systems have been extensively studied in the recent past in order to construct artificial light harvesting systems that mimic nature. However, this is the first report where a semiconductor quantum dot has been used as a sensitizer for such studies.

\section{Experimental Section}

\section{Insertion of Thionine and Oxonine into zeolite $\mathrm{L}$}

Cationic dye molecules were loaded into zeolite L crystals by ion exchange from water. In a typical experiment, $50 \mathrm{mg}$ of zeolite crystals were suspended in $2 \mathrm{~mL}$ doubly deionized water. The number of sites present in the sample is given by the total number of zeolite unit cells divided by the number of unit cells one guest molecule occupies:

$$
\mathrm{N}_{\mathrm{s}}=\left(\mathrm{m}_{\mathrm{z}} / 2880 \mathrm{~g} / \mathrm{mol}\right) * 1 / \mathrm{S}
$$

where $\mathrm{m}_{\mathrm{z}}$ is the weight of the zeolite L sample in grams, $2880 \mathrm{~g} / \mathrm{mol}$ is the molecular weight of a unit cell, and $S$ is the number of unit cells one dye molecule occupies. For both thionine and oxonine, $\mathrm{S}=1.5 . \mathrm{N}_{\mathrm{s}}$ was calculated 
to be $1.15 \times 10^{-5}$. The amount of dye molecules (to be inserted) is the product of $\mathrm{N}_{\mathrm{s}}$ and the desired loading percentage. This leads to $5.7 \times 10^{-7}$ moles for a $5 \%$ loading of thionine, corresponding to about $12 \mu \mathrm{g}$. This amount of thionine was added to the zeolite suspension, and then the suspension was sonicated for 15 minutes followed by 12 hours of reflux. After centrifugation, the dye-zeolite $\mathrm{L}$ sample was washed two times with ethanol to get rid of dyes physisorbed on the crystal surface.

The loading with oxonine was carried out in a similar way. About $12 \mu \mathrm{g}(0.57$ $\mu \mathrm{mol})$ of oxonine was added to a suspension of $50 \mathrm{mg}$ of zeolites in water, and subsequently sonicated for 15 minutes. The mixture was refluxed for 12 hours. The dye loaded zeolites were washed with ethanol twice to get rid of any adsorbed dye molecules. Both the samples were thoroughly dried in a hot air oven overnight before using for any photophysical measurements.

\section{Photophysical Measurements}

Steady-state emission measurements were performed using a HORIBA JobinYvon IBH FL-322 Fluorolog 3 spectrometer equipped with a $450 \mathrm{~W}$ xenon arc lamp, double grating excitation and emission monochromators and a TBX-4-X single-photon-counting detector. For the sensitization measurements, the emission was recorded with front face geometry and the sample was stirred at $450 \mathrm{rpm}$ during the measurement in order to ensure minimum precipitation of the zeolite crystals in toluene. Confocal microscopy measurements were performed on a Microtime 200 confocal microscope from Picoquant. Samples with a zeolite concentration of $1 \mathrm{mg} / \mathrm{mL}$ in toluene, with and without quantum dots added, were drop cast onto a glass cover slip and the solvent was allowed to evaporate and single crystal measurements were performed. 


\section{References}

1. Cram, D. J., Angew. Chem. Int. Ed. 1988, 27, 1009.

2. Pedersen, C. J., Angew. Chem. Int. Ed. 1988, 27, 1021.

3. Yeates, T. O.; Padilla, J. E., Curr. Opin. Struct. Biol. 2002, 12, 464.

4. Szejtli, J., Chem. Rev. 1998, 98, 1743.

5. Newkome, G. R., Pure \& Appl. Chem. 1998, 70, 2337.

6. Zimmerman, S. C.; Wang, Y.; Bharathi, P.; Moore, J. S., J. Am. Chem.Soc. 1998, 120, 2172.

7. Lehn, J.-M., Polym. Int. 2002, 51, 825.

8. Dulebohn, J. I.; Van Vlierberge, B.; Berglund, K. A.; Lessard, R. B.; Yu, J. A.; Nocera, D. G., Mater. Res. Soc. Symp. Proc. 1990, 180, 733.

9. St. John, J.; Coffer, J. L.; Chen, Y.; Pinizzotto, R. F., J. Am. Chem.Soc. 1999, 121, 1888.

10. Heikenfeld, J.; Garter, M.; Lee, D. S.; Birkhahn, R.; Steckl, A. J., Appl. Phys. Lett. 1999, 75, 1189.

11. Wöhrle, D.; Schulz-ekloff, G., Adv. Mater. 1994, 6, 875.

12. Caro, J.; Marlow, F.; Wübbenhorst, M., Adv. Mater. 1994, 6, 413.

13. Ramamurthy, V., J. Photochem. Photobiol., C 2000, 1, 145.

14. Baerlocher, C.; McCusker, L. B.; Olson, D. H., In Atlas of Zeolite Framework Types, Elsivier: Amsterdam, 2007.

15. Megelski, S.; Calzaferri, G., Adv. Funct. Mater. 2001, 11, 277.

16. Ernst, S.; Weitkamp, J., Catal. Today 1994, 19, 27.

17. Lovallo, M. C.; Tsapatsis, M., In Advanced Catalysts and Nanostructured Materials, William, R. M., Ed. Academic Press: San Diego, 1996; pp 307.

18. Newsam, J. M., J. Phys.Chem. 2002, 93, 7689.

19. Tsapatsis, M.; Okubo, T.; Lovallo, M. C.; Davis, M. E., Mater. Res. Soc. Symp. Proc. 1995, 371, 21.

20. Tsapatsis, M.; Lovallo, M.; Okubo, T.; Davis, M. E.; Sadakata, M., Chem. Mater. 2002, 7, 1734.

21. Rollmann, L. D.; Valyocsik, E. W.; Shannon, R. D., Zeolite Molecular Sieves. In Inorganic Syntheses, Donald W. Murphy, L. V. I., Ed. 2007; pp 227. 
22. Hashimoto, S.; Ikuta, S.; Asahi, T.; Masuhara, H., Langmuir 1998, 14, 4284.

23. Liu, X.; Thomas, J. K., Chem. Mater. 2002, 6, 2303.

24. Corrent, S.; Hahn, P.; Pohlers, G.; Connolly, T. J.; Scaiano, J. C.;

Fornes, V.; Garcia, H., J. Phys.Chem. B. 1998, 102, 5852.

25. Calzaferri, G.; Gfeller, N., J. Phys.Chem. 1992, 96, 3428.

26. Ramamurthy, V.; Sanderson, D. R.; Eaton, D. F., J. Am. Chem.Soc. 1993, 115, 10438.

27. Yoon, K. B.; Huh, T. J.; Corbin, D. R.; Kochi, J. K., J. Phys.Chem. 1993, 97, 6492 .

28. Ganesan, V.; Ramaraj, R., J. Lumin. 2001, 92, 167.

29. Pauchard, M.; Devaux, A.; Calzaferri, G., Chem. Eur. J. 2000, 6, 3456.

30. Hashimoto, S.; Hagiri, M.; Matsubara, N.; Tobita, S., Phys. Chem. Chem. Phys. 2001, 3, 5043.

31. Maas, H.; Calzaferri, G., Angew. Chem. Int. Ed. 2002, 41, 2284.

32. Khatyr, A.; Maas, H.; Calzaferri, G., J. Org. Chem. 2002, 67, 6705.

33. Calzaferri, G.; Huber, S.; Maas, H.; Minkowski, C., Angew. Chem. Int. Ed. 2003, 42, 3732.

34. Calzaferri, G.; Maas, H.; Pauchard, M.; Pfenniger, M.; Megelski, S.; Devaux, A., Adv. Photochem. 2002, 27, 1.

35. Calzaferri, G.; Pauchard, M.; Maas, H.; Huber, S.; Khatyr, A.; Schaafsma, T., J. Mater. Chem. 2002, 12, 1.

36. Devaux, A.; Popovic, Z.; Bossart, O.; De Cola, L.; Kunzmann, A.; Calzaferri, G., Micropor. Mesopor. Mater. 2006, 90, 69.

37. Albuquerque, R. Q.; Popovi, Z.; cacute; Cola, L. D.; Calzaferri, G., Chem. Phys. Chem. 2006, 7, 1050.

38. Kongkanand, A.; Tvrdy, K.; Takechi, K.; Kuno, M.; Kamat, P. V., J. Am. Chem. Soc. 2008, 130, 4007.

39. Leschkies, K. S.; Divakar, R.; Basu, J.; Enache-Pommer, E.; Boercker, J. E.; Carter, C. B.; Kortshagen, U. R.; Norris, D. J.; Aydil, E. S., Nano Lett. 2007, 7, 1793.

40. Lopez-Luke, T.; Wolcott, A.; Xu, L. p.; Chen, S.; Wen, Z.; Li, J.; DeLaRosa, E.; Zhang, J. Z., J. Phys. Chem. C 2008, 112, 1282.

41. Alivisatos, A. P., J. Phys. Chem. 1996, 100, 13226. 
42. Alivisatos, A. P., Science 1996, 271, 933.

43. Klimov, V. I., Semiconductor and Metal Nanocrystals. Synthesis and Electronic and Optical Properties. Marcel Dekker: New York, 2004; p 159

44. Nirmal, M.; Brus, L., Acc. Chem. Res. 1999, 32, 407.

45. Ohsuna, T.; Horikawa, Y.; Hiraga, K.; Terasaki, O., Chem. Mater. 1998, 10, 688.

46. Huber, S. Harvesting Electronic Excitation in Host Guest Materials for Light Energy Conversion. Universität Bern, Bern, 2005.

47. Yu, W. W.; Qu, L.; Guo, W.; Peng, X., Chem. Mater. 2003, 15, 2854.

48. Dexter, D. L., J. Chem. Phys. 1953, 21, 836.

49. Förster, T., Ann. Physik 1948, 2, 55.

50. Förster, T., In Fluorezenz. Organischer Verbindungen, Vandenhoech \& Ruprech: Göttingen, 1951.

51. Förster, T., Disc. Faraday Soc. 1959, $27,7$.

52. Lackowicz, J. R., In Principles of Fluorescence Spectroscopy, 3rd ed.; Springer 2006; pp 281. 


\section{Summary}

Nanocrystal quantum dots (NQDs) are an exciting class of materials that offer a unique possibility to tailor their optoelectronic properties due to their size dependent behavior originating from quantum confinement of charge carriers. This particular aspect imparts on them significantly property differences compared to their bulk counterparts, providing numerous important opportunities in the field of nanoscale semiconductor science. One of the biggest challenges from an application point of view is to increase their processability (both in solid state and in solution phase) and to interface them with a matrix, or molecules that are able to provide electronic energy or charges. Various approaches have been used and adapted in this regard such as incorporating these materials in a polymer matrix (for OLED applications) or combining them with mesoporous $\mathrm{TiO}_{2}$ (for photovoltaic applications).

Our approach is to functionalize these materials with (electro)luminescent metal complexes based on $\operatorname{Ir}(\mathrm{III})$ and $\mathrm{Ru}$ (II) metal ions, coordinated with ligands possessing appropriate anchoring groups that will interact with the surface of the quantum dot. These metal complexes are chosen as they are redox active chromophores with very rich photophysics (particularly emissive triplet states and electroluminescence) and electrochemistry (excellent charge transfer properties) and hence are excellent candidates for optoelectronic applications. By combining these two different classes of materials, one could fabricate a bifunctional nanoassembly with a possibility to control the band gap properties of both the constituent fragments synergistically. Understanding of basic photophysical processes that occur in such materials are of quintessential importance for the development of practical applications and further design. This thesis deals with the design of assemblies incorporating quantum dots and organic or organometallic chromophores. The photoinduced processes in such organic-inorganic hybrid nanosystems are investigated.

The thesis is divided in 6 chapters which are briefly described below.

Chapter 1 is a general introduction to the properties of quantum dots, metal complex photophysics and finally the possible photoinduced processes that can occur between a donor and an acceptor pair. For this reason it is divided into three sections. The first section deals with the fundamental optical 
properties of quantum dots. Quantum confinement effects of charge carriers on the optical properties of these nanocrystals are discussed. Surface properties have a significant impact on the spectroscopic properties of these nanoparticles. A brief summary on the surface - ligand interaction that is exploited in the current work to exchange the organic ligands with the metal complexes is presented. Basic photophysical properties of the transition metal complexes are discussed in the second section of the chapter, giving the reader sufficient background to understand more complex phenomena. In the final section of chapter 1, the fundamental aspects of photoinduced energy and electron transfer processes are illustrated, in particular the possible mechanisms for electronic energy transfer (Förster and Dexter principles) and charge transfer using Marcus theory. Only basic processes which will be encountered in subsequent chapters to explain the photoinduced properties of the active components are focused upon. All the spectroscopic and microscopic techniques, as well as the electrochemical techniques and time resolved measurements employed to probe the nanoscale assemblies are discussed in chapter 2 of this dissertation.

In chapter 3, interactions between chromophoric systems and NQD are described and evidences of photoinduced electronic energy transfer between metal complexes and NQD conjugates are probed. Specifically, two luminescent metal complexes containing $\operatorname{Ir}(\mathrm{III})$ and $\mathrm{Ru}(\mathrm{II})$ metal ions with amino functionality as an anchoring group for NQDs have been used to demonstrate an electronic interaction between the metal complexes and the quantum dots. The first system in this study consists of a monocationic $\operatorname{Ir}(\mathrm{III})$ complex, coordinated by two phenypyridine and one bipyridine substituted in the 4 position with a biphenyl amino group. Through an interaction via amino functionality, the metal complexes are assembled onto red emitting CdTe nanocrystals. Exciting the donor (iridium complex) in the nanoassembly at 400 $\mathrm{nm}$ resulted in photoinduced resonance energy transfer from the $\operatorname{Ir}(\mathrm{III})$ fragment to the quantum dots; this is schematically represented in figure S1. Energy transfer is confirmed by the quenching of the orange iridium emission and sensitization of the red CdTe emission. 


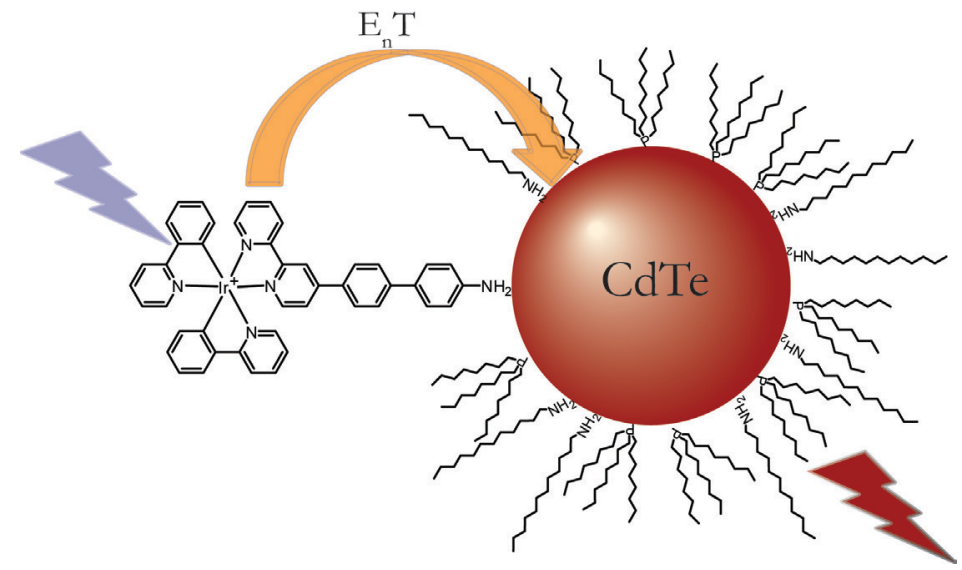

S1: Schematic representation of resonance energy transfer from Ir(III) to NQD

In order to demonstrate that the energy transfer between NQDs and metal complexes maybe bidirectional, we have investigated a $\mathrm{Ru}(\mathrm{II})$ complex containing the same amino terminated bipyridine derivative bound to $\mathrm{CdSe} / \mathrm{ZnS}$ NQD surface through a $\mathrm{CS}_{2}$ unit. In this system, due to the higher excited state of the quantum dots vs. the complex, sensitization occurs from the CdSe/ZnS NQDs to the $\mathrm{Ru}(\mathrm{II})$ fragments. Förster resonance energy transfer (FRET) occurs from the NQDs to the Ru(II) units upon excitation at $431 \mathrm{~nm}$; this is schematically illustrated in figure S2.

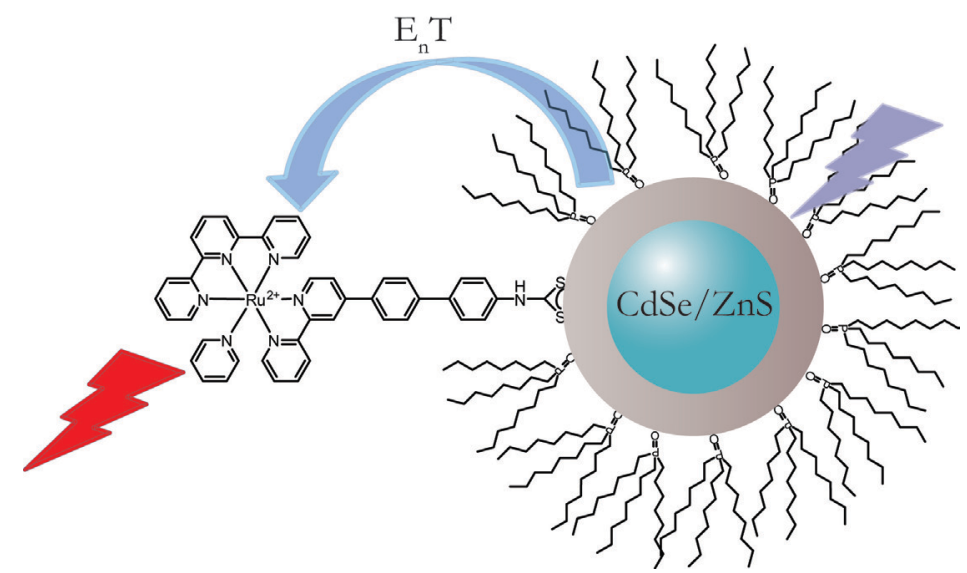

S2: Schematic representation of resonance energy transfer from NQD to $\mathrm{Ru}(\mathrm{II})$ 
Even though the amino group provides a good anchoring moiety for the NQDs we have designed a metal complex with multiple anchoring units that can bind to the surface of the quantum dots. Chapter 4 discusses the properties of ruthenium complexes with tripodal geometries bearing acetyl thiol and tertiary butyl functionalities prepared in collaboration with Prof. Belser. In particular the photophysical and electrochemical characterization of these complexes in solution are described. To demonstrate the versatility of such tripodal molecular structures, self assembled monolayer of the thiolated ruthenium complex on gold and platinum surfaces have been investigated. Furthermore, a metal-molecule-metal junction with the monolayer is constructed using a eutectic of gallium-indium alloy (EGaIn) as top contact and gold as the bottom electrode. Rectification behavior is shown in such a junction upon charge injection through top and bottom electrodes. This system is interesting for potential applications in molecular electronics.

Once established that the molecules can strongly bind to metallic surfaces and support charge transport, the behavior of these tripodal ruthenium complexes bound to the quantum dot surface was explored; this is described in chapter 5 . Green emitting CdTe quantum dots were employed with thiol-substituted $\mathrm{Ru}(\mathrm{II})$ complexes to form the nanoassembly. Contrary to that shown in chapter 3, where core/shell CdSe/ZnS NQDs were used, upon excitation of the CdTe NQDs, quenching of both components is observed. Upon excitation at $500 \mathrm{~nm}$ (exciton absorption maximum), excitons are formed in the NQD core. Several processes at this point take place with different kinetics and spectral characteristics. In order to understand all the consecutive processes, subpicosecond and nanosecond transient absorption and time resolved emission spectroscopies have been employed. The overall picture shows that the intraband relaxation of the hot carriers to the band-edge is suppressed due to the presence of the thiol-substituted metal complexes, but the relaxation eventually takes place through an energy transfer to the $\mathrm{Ru}$ (II) moiety. From the band edge, an electron transfer to the $\mathrm{Ru}(\mathrm{II})$ fragment is observed. The recombination of the charge separated state occurs in 325 ns leading to the original ground state. The understanding of all the processes and the design of 
suitable systems possessing a high efficiency of the charge transport is essential for photovoltaic applications, where harnessing the excitons is a fundamental step. Figure S3, represents a schematic energy level diagram summarizing all the processes described in chapter 5 .

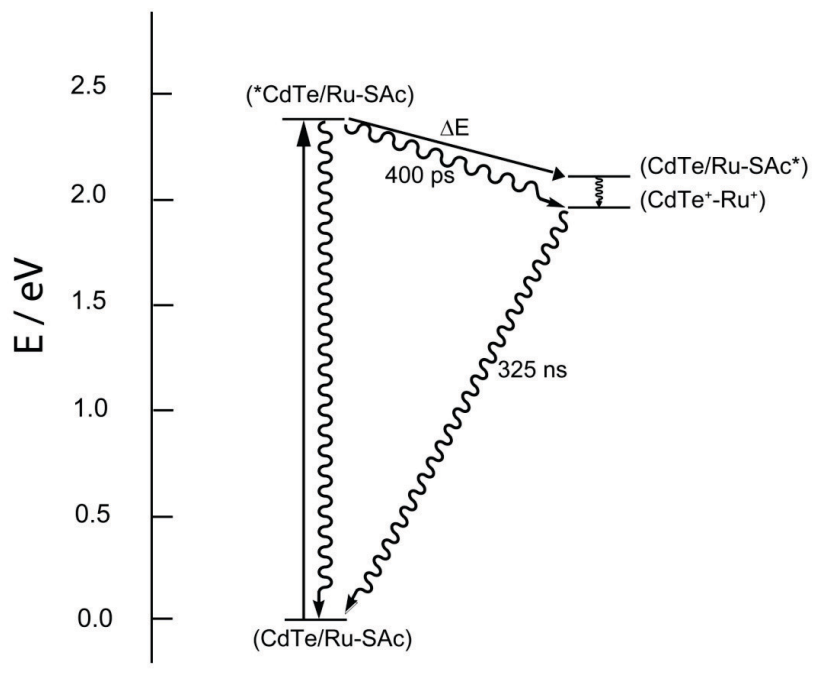

S3: Schematic representation of photoinduced electron transfer between CdTe NQD and thiol-substituted $\mathrm{Ru}$ (II) complex.

Assembling molecules to nanoparticles is a rather established field of nanoscience. We decided to go a step further and look at the possibility to link inert nanocontainers to NQDs and study the possible processes between dye molecules entrapped in the nanocontainers and the semiconductor nanocrystals. Chapter 6 deals with the resonance energy transfer between NQDs and a host-guest system. After filling the channels of zeolite L crystals of $1 \mu \mathrm{m}$ length, with low energy absorbing and emitting dye molecules, oxonine and thionine, the zeolites have been decorated with yellow emitting $\mathrm{CdSe} / \mathrm{ZnS}$ dots. Upon selectively exciting the quantum dots, a vectorial energy transfer from the dots to the dye molecules inside the zeolite channels is observed. This can be monitored by confocal emission and time resolved microscopy following the quenching of the quantum dots lifetimes and the 
sensitization of the entrapped dye molecules. The process is schematically shown in figure S4. This system demonstrates the feasibility to utilize NQDs in combination with host-guest systems for potential light harvesting applications.

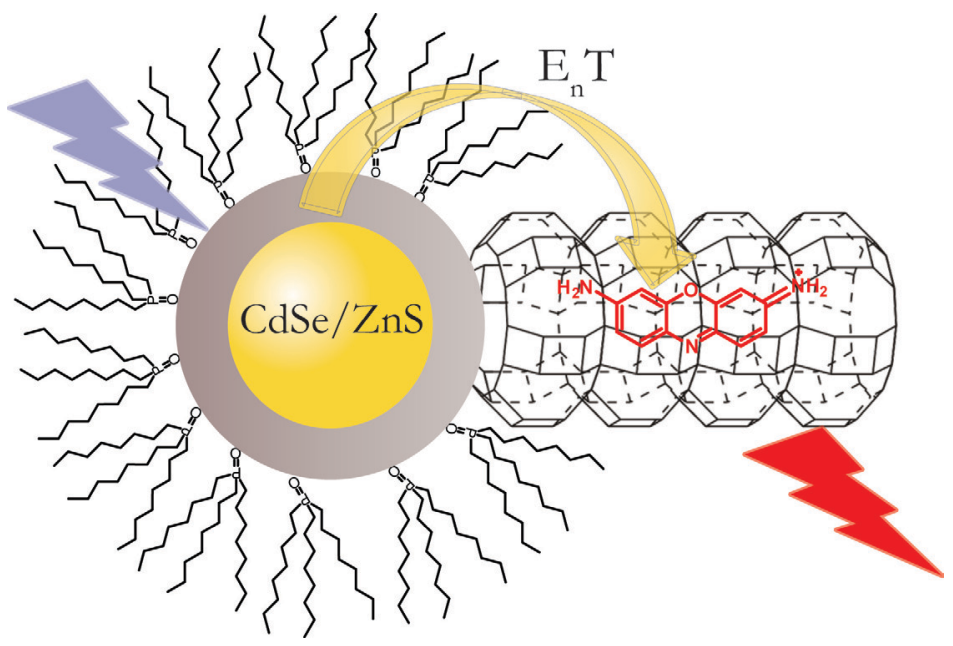

S4: Schematic representation of resonance energy transfer from CdSe/ZnS NQD to dye molecule inside the channel of zeolite $\mathrm{L}$ 


\section{Samenvatting}

Quatumdots van nanokristalen (NQDs) zijn een opwindende groep van materialen die een unieke mogelijkheid bieden om hun optoelectronische eigenschappen aan te passen gezien ze grootte-afhankelijkheid gedrag uit kwantum opsluiting van ladingsdragers vertonen. Dit bepaalde aspect verleent op hen beduidende verschillen in eigenschappen in vergelijking met hun bulk tegenhangers, die talrijke belangrijke mogelijkheden op het gebied van nanoschaal halfgeleider wetenschap bieden. Één van de grootste uitdagingen vanuit een toepassingsstandpunt is het uitbreiden van hun verwerkbaarheid (zowel in vaste toestand als in oplossing) en het "interfacen" met een matrix of met moleculen die in staat zijn om electronische energie of ladingen te verstrekken. In dit verband, diverse benaderingen zijn gebruikt en aangepast, zoals het opnemen van deze materialen in een polymeermatrix (voor OLED toepassingen) of het combineren van hen met mesoporeuze $\mathrm{TiO}_{2}$ (voor fotovoltaïsche toepassingen).

Onze benadering is het functionaliseren van deze materialen met (electro) luminescerende metaalcomplexen gebaseerd op $\mathrm{Ir}(\mathrm{III})$ en $\mathrm{Ru}(\mathrm{II})$ metaalionen, gecoördineerd met liganden welke geschikte verankerende groepen bezitten die een interactie met het oppervlak van de quantumdot aan zullen gaan. Deze metaalcomplexen worden gekozen aangezien zij redox actieve chromoforen zijn met zeer rijke fotofysica (in het bijzonder emitterende tripet toestanden en electroluminescentie) en electrochemie (uitstekende ladingsoverdracht eigenschappen) en daardoor zijn ze uitstekende kandidaten voor optoelectronische toepassingen. Door het combineren van deze twee verschillende materialen kon men bifunctionele nano-assemblages fabriceren met de mogelijkheid tot synergetische controle van de bandafstand eigenschappen van beide constituerende fragmenten. Het begrijpen van fundamentele fotofysische processen die in dergelijke materialen voorkomen is van groot belang voor de ontwikkeling van praktische toepassingen en verder ontwerp. Dit proefschrift behandelt het ontwerpen van assemblages van quantumdots en organische of organometallische chromoforen. De 
fotogeïnduceerde processen in dergelijke organisch-anorganische hybride nanosystemen worden onderzocht.

Dit proefschrift is verdeeld in 6 hoofdstukken die in het kort hieronder worden beschreven.

Hoofdstuk 1 is een algemene inleiding m.b.t. de eigenschappen van quantumdots, metaalcomplex fotofysica en de mogelijk fotogeïnduceerde processen die tussen een donor en een acceptor paar plaats kunnen vinden. Om deze reden is het hoofdstuk verdeeld in drie secties. In de eerste sectie worden de fundamentele optische eigenschappen van quantumdots behandeld. De gevolgen m.b.t. kwantum opsluiting van ladingsdragers op de optische eigenschappen van deze nanokristalen worden besproken. De oppervlakeigenschappen hebben een significante invloed op de spectroscopische eigenschappen van deze nanodeeltjes. Een korte samenvatting m.b.t. de oppervlak-ligand interactie die in het huidige werk geëxploiteerd is om organische liganden met metaalcomplexen uit te wisselen wordt voorgesteld. De fundamentele fotofysische eigenschappen van de overgangsmetaalcomplexen worden besproken in de tweede sectie van dit hoofdstuk, zodat de lezer voldoende achtergrondinformatie krijgt om complexere fenomenen te begrijpen. In de laatste sectie van hoofdstuk 1, de fundamentele aspecten van fotogeïnduceerde energie en electronoverdracht processen worden geillustreerd, in het bijzonder de mogelijke mechanismen voor electronische energieoverdracht (Förster en Dexter principes) en ladingsoverdracht gebruikmakend van de theorie van Marcus. Slechts de basisprocessen die in verdere hoofdstukken zullen worden gehanteerd om de fotogeïnduceerde eigenschappen van de actieve componenten te verklaren worden behandeld. Alle spectroscopische en microscopische technieken, evenals electrochemische technieken en time-resolved metingen die gebruikt werden om de nanoschaal assemblages te sonderen worden besproken in hoofdstuk 2 van dit proefschrift.

In hoofdstuk 3 worden de interacties tussen chromofoor-gebaseerde systemen en NQD beschreven en het bewijsmateriaal m.b.t. fotogeïnduceerde electronische energieoverdracht tussen metaalcomplexen en de gepaarde 
NQDs wordt gesondeerd. In het bijzonder, twee luminescerende metaalcomplexen van $\operatorname{Ir}(\mathrm{III})$ and $\mathrm{Ru}(\mathrm{II})$ met amino-functionaliteiten als verankerende groepen voor NQDs werden gebruikt om een electronische interactie tussen de metaalcomplexen en de quantumdots aan te tonen. Het eerste systeem in deze studie bestaat uit een mono-kationisch $\operatorname{Ir}(\mathrm{III})$ complex, gecoördineerd door twee fenylpyridines en één bipyridine gesubstitueerd met een bifenylamine groep in de $4^{\text {de }}$ positie. Door een interactie via de aminofunctionaliteit, worden er metaalcomplexen geassembleerd tot roodemitterende CdTe nanokristalen. Excitatie van de donor (complex van iridium) in de nano-assemblage bij $400 \mathrm{~nm}$ resulteerde in fotogeïnduceerde resonantieenergieoverdracht van het $\operatorname{Ir}(\mathrm{III})$ fragment naar de quantumdots; dit wordt schematisch weergegeven in figuur S1. De energieoverdracht wordt bevestigd door de quench van de oranje iridiumemissie en de sensibilisatie van de rode CdTe emissie.

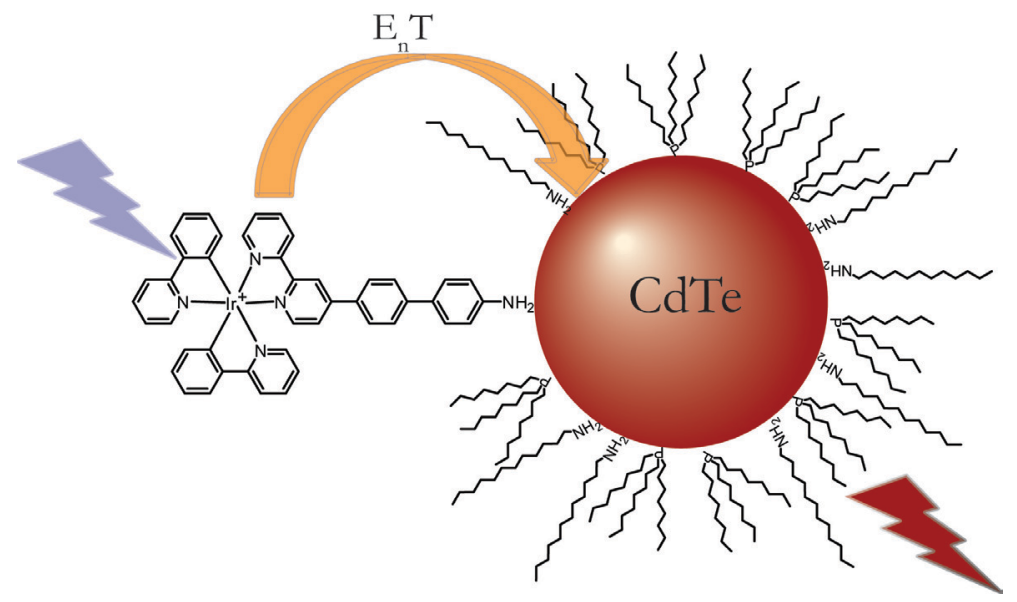

S1: Schematische weergave van de resonantie-energieoverdracht van $\operatorname{Ir}(\mathrm{III})$ naar NQD

Om aan te tonen dat de energieoverdracht tussen NQDs en de metaalcomplexen waarschijnlijk bidirectioneel is, hebben wij een $\mathrm{Ru}(\mathrm{II})$ complex, die hetzelfde amino bipyridinederivaat verbonden aan de CdSe/ZnS NQD oppervlak door een $\mathrm{CS}_{2}$ eenheid bevat, onderzocht. In dit systeem, 
wegens de hogere aangeslagen toestand van de quantumdots versus het complex, vindt de sensibilisering plaats van de CdSe/ZnS NQDs naar de $\mathrm{Ru}(\mathrm{II})$ fragmenten. De resonantie-energieoverdracht van Förster (FRET) vindt plaats van de NQDs naar de Ru(II) eenheden na excitatie bij $431 \mathrm{~nm}$; dit wordt schematisch weergegeven in figuur S2.

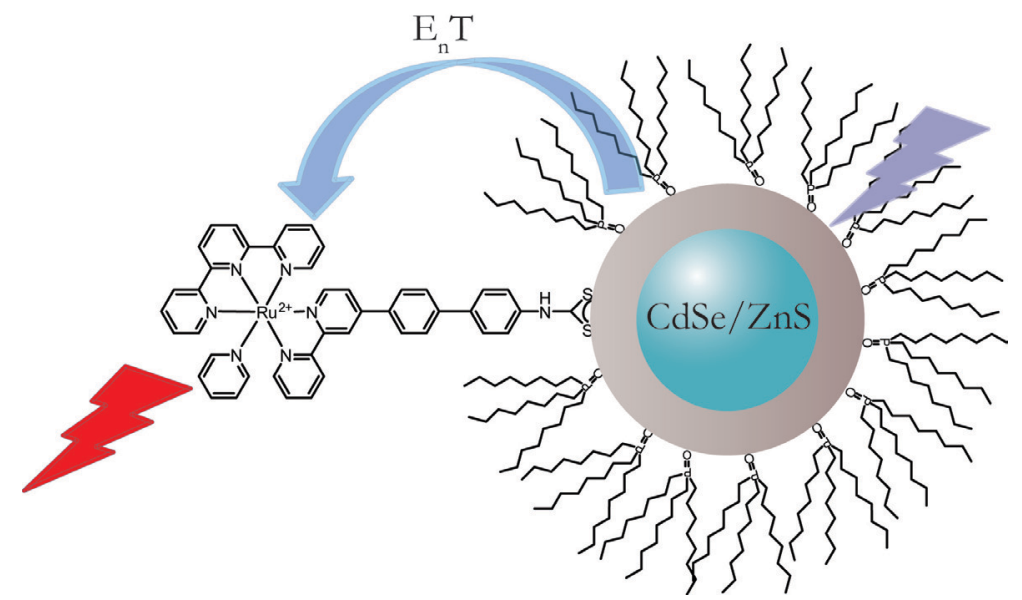

S2: Schematische weergave van de resonantie-energieoverdracht van NQD naar $\mathrm{Ru}(\mathrm{II})$

Ondanks het feit dat de aminogroepen een goed verankerend "moeity" voor de NQDs zijn, hebben wij een metaalcomplex met veelvoudige verankerende eenheden ontworpen die aan de oppervlak van de quantumdots kan binden. In hoofdstuk 4 worden de eigenschappen van rutheniumcomplexen met een tripodal geometrie besproken die acetyl thiol en tertiaire butyl-functionaliteiten bevatten en die in samenwerking met Prof. Belser werden gemaakt. In het bijzonder wordt er de fotofysische en electrochemische karakterisering van deze complexen in oplossing beschreven. Om de veelzijdigheid van dergelijke tripodal moleculaire structuren aan te tonen, werden er zelf-geassembleerde monolagen van het thiol bevattend rutheniumcomplex op goud- en platinaoppervlakken onderzocht. Daarnaast, wordt er een metaal-molecuulmetaal grenslaag met de monolaag gemaakt gebruikmakend van een eutectische gallium-indium legering (EGaIn) als "bovenste" contact en goud als 
"onderste" electrode. Het gedrag van de rectificatie in de grenslaag wordt getoond na de ladingsinjectie door de bovenste en onderste electroden. Dit systeem is interessant voor potentiële toepassingen in moleculaire elektronica.

Zodra er vastgesteld werd dat de moleculen zich sterk aan metaaloppervlaktes kunnen binden en ladingstransport ondersteunen, werd het gedrag van deze tripodal rutheniumcomplexen verbonden aan de quantumdotoppervlak onderzocht; dit wordt beschreven in hoofdstuk 5. Groen-emitterende CdTe quantumdots met thiol-gesubstitueerde $\mathrm{Ru}(\mathrm{II})$ complexen werden gebruikt om nano-assemblages te vormen. In tegenstelling tot wat er aangetoond werd in hoofdstuk 3, waar kern/schil CdSe/ZnS NQDs werden gebruikt, werd na excitatie van CdTe NQDs een quench van beide componenten waargenomen. $\mathrm{Na}$ excitatie bij $500 \mathrm{~nm}$ (exciton absorptiemaximum), worden er excitonen gevormd in de NQD kern. Op dit punt vinden er meerdere processen plaats met verschillende kinetiek en spectrale kenmerken. Om alle opeenvolgende processen te begrijpen, werd er subpico- en nanoseconde transiënt-absorptie en time-resolved emissiespectroscopie gebruikt. Het algemene beeld toont aan dat de intraband relaxatie van de "hot carriers" aan de bandrand wordt onderdrukt door de aanwezigheid van de thiol-gesubstitueerde metaalcomplexen. Echter, de relaxatie vindt uiteindelijk plaats door een energieoverdracht naar het $\mathrm{Ru}(\mathrm{II})$ deel. Van de bandrand, wordt er een electronoverdracht aan het $\mathrm{Ru}(\mathrm{II})$ fragment waargenomen. De recombinatie van de lading-gesplitste toestand gebeurt in $325 \mathrm{~ns}$, wat tot de originele grondtoestand leidt. Het begrijpen van alle processen en ontwerp van geschikte systemen die een hoge efficiëntie van het ladingsoverdracht bezitten is essentieel voor fotovoltaïsche toepassingen, waar excitonen een fundamentele rol spelen. Figuur S3 weergeeft het energieniveau diagram dat alle processen die in hoofdstuk 5 worden beschreven samenvat. 


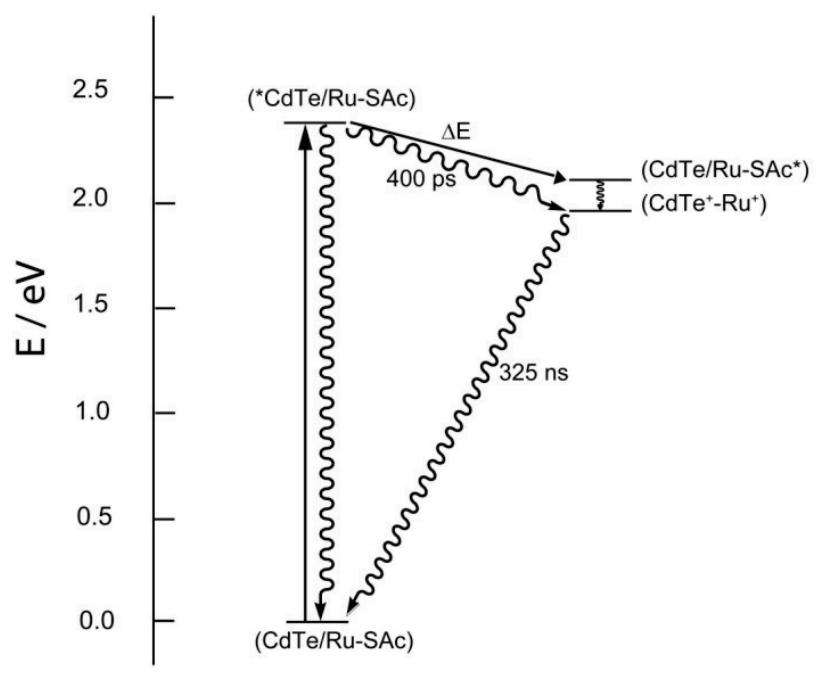

S3: Schematische weergave van de fotogeïnduceerde electronoverdracht tussen CdTe NQD en het thiol-gesubstitueerde Ru(II) complex.

Het assembleren van moleculen tot nanodeeltjes is een erkend gebied van de nanowetenschap. Wij beslisten om een stap verder te gaan en de mogelijkheid te bekijken om inerte nanocontainers te binden aan NQDs en de mogelijke processen tussen kleurstofmoleculen die in nanocontainers gevangen zijn en halfgeleider nanokristalen te bestuderen. Hoofdstuk 6 behandelt de resonantie-energieoverdracht tussen NQDs en een host-guest systeem. $\mathrm{Na}$ het vullen van de $1 \mu \mathrm{m}$ lengte kanalen van de kristallen van zeoliet $\mathrm{L}$ met lage energieabsorberende en emitterende kleurstofmoleculen, oxonine en thionine, zijn de zeolieten gedecoreerd met geel-emitterende CdSe/ZnS dots. Als de quantumdots selectief worden geëxciteerd, is een "vectorial" energieoverdracht van de dots naar de kleurstofmolecules binnen de zeolietkanalen waargenomen. Dit kan door confocal-emissie en time-resolved microscopie worden gecontroleerd na de quench van de quantumdot levensduur en de sensibilisering van de gevangen kleurstofmoleculen. Het proces wordt schematisch weergegeven in figuur S4. Dit systeem toont de uitvoerbaarheid aan van het gebruik van NQDs in combinatie met host-guest systemen voor potentiële light harvesting toepassingen. 


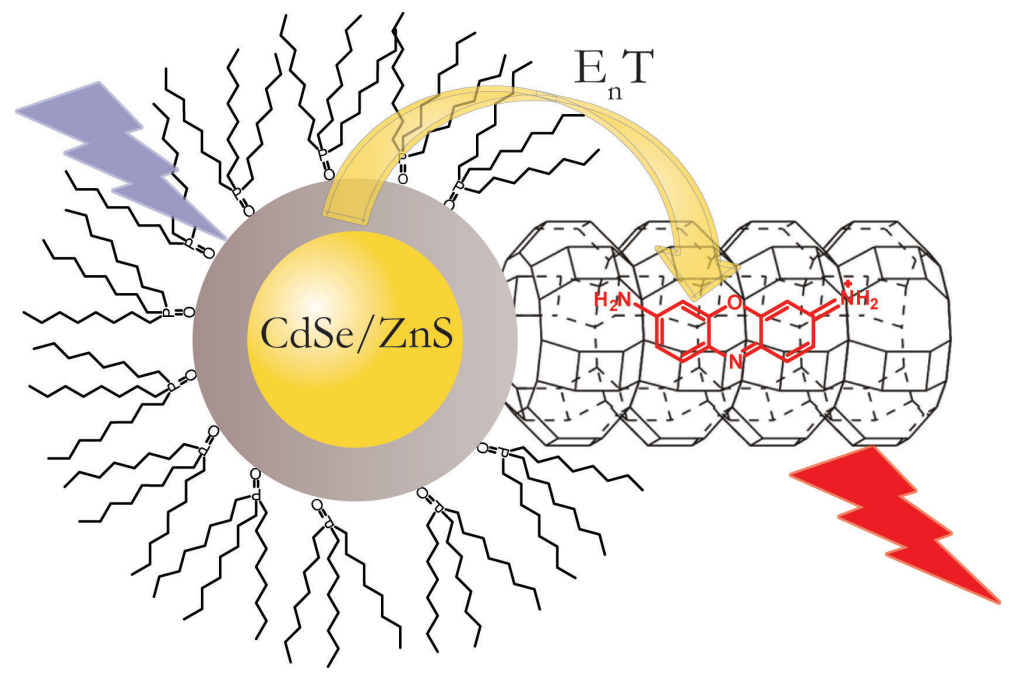

S4: Schematische weergave van de resonantie-energieoverdracht van CdSe/ZnS NQD naar het kleurstofmolecuul binnen het kanaal van zeoliet L. 


\section{Acknowledgments}

It has been a long and eventful journey. Finally I arrive at the part of my thesis where I like to thank all the people who made my $\mathrm{PhD}$ days eventful during the last 4 years. First of all, I would like to thank Prof. Luisa De Cola, my promoter, for giving me an opportunity to work on a very interesting project and in a wonderful group. Thank you, Luisa, for this opportunity and all the support that you provided me. I would like to thank Prof. David Reinhoudt, my promoter, for accommodating me in his group. Thank you, David, for all the support that you provided me.

During my relatively shorter stay at SMCT, I received good support from Dr. Aldrik Velders. Thank you, Aldrik, for all the formal and informal support. I thank Prof. Wilfred van der Wiel for some interesting discussions about my work. I also would like to thank Prof. Jurriaan Huskens.

I was fortunate to be involved in some very good collaboration with various people. I would like to thank Prof. Daniel Vanmaekelbergh and Dr. Rolf Koole for a wonderful collaboration. I would like to thank Prof. Peter Belser and Dr. Fabio Edaffe for very nice collaboration. I would also thank Dr. Chris Nijhuis and Prof. George Whitesides for an exciting collaboration. I thank Dr. Rene Williams and Dr. Pablo Contreras Carballada for a fruitful collaboration.

Majority of my $\mathrm{PhD}$ work was carried out in Münster, Germany. I feel extremely lucky to be a part of the 'amazing' Photonics group. In my journey in last 4 years, I have met a lot of interesting and wonderful people. All these people made my $\mathrm{PhD}$ a memorable one. I would like to thank all of them for the help I got and being so welcoming to me.

Klaus, from the first day in Münster, you have been very helpful. I just can't thank you enough. In you, I not only found a wonderful coworker, but also one of the best friends. Thank you, Klaus for all the wonderful moments that we have shared. Gregg, thanks for being my informal supervisor during my early years in Münster. It was a pleasure working with you. 
Ásgeir, you are truly amazing, Zoran, thanks for being a good friend, collaborator and a host (in Boston), Enrico (I still remember my first day taking out solvents with you), Marita (my cuvette partner ;)), Gabri, Mike, Manuel (Schnukie! My multifunctional buddy!), Fabio (my Italian teacher), Sandra (javol Sandra speaking), Yolanda, Silvia, Miriam, Yinghui (Panda), Andrés (Our Madrid tourist guide), Cristian (I had a great time with the energy transfer discussion), David, Benoit, Rodrigo, André, (always on time Swiss guy), Gigi (or DXP), Aurélie, Pablo (thanks for your help in Amsterdam) Victor, Sara and Carlo. Mathias Mydlak (my great German teacher, check!!), Matthias Otter (meine ausländer freund), Claudia (the only Italian I know who does not swear :)), Jesus (Sponge bob), Lola, Federico, Matteo (my African friend ;)), Cheng-Han (Bruce almighty), Chen-Han, Novian (Prof. Darmawan), Sebastian (Dück stück), Eike, Edward (I am looking forward to your India trip), Lenny, Christine, Zhen, Naveen, Jan, Yan, Yugui, Andrea, Seda, Mark, Moema, Henning, Malte. Thank you all.

Thanks to Insa, Nicole, Bernhard, Melanie and Mrs. Maria Jaklin for all the support. Special thanks to Aldrik, André, Edward, Christine, Lenny, Mathias, Sandra, Matthias, Manuel, Fabio and Sebastian for proof reading my thesis chapters.

During my short stay in SMCT, I met a lot of people who were very helpful. First of all I would like to thank Richard and Marcel for all the technical help. It would have been very difficult for me but for their never ending help. I would also thank Tieme, Ben and Bianca for their help. Of course I am extremely thankful to Izabel, Geradine and Danielle for helping me with all the bureaucratic works. I would extend my thanks to Dr. Willem Verboom and Dr. Pascal Jonkheijm.

I would like to convey my heartfelt thanks to Jealemy for the timely Dutch translation of the summary of my thesis. Thank you, Jealemy, also for being a wonderful friend. I would like to thank Sachin, Veera, Kim (Hey dude! Did you see the match?)), Ignacio (Nachete), Francesca, Ricardo (my photo critique), Albert (mio amico), Arancha, Alberto, Henk, Martine, Shu Han, 
Xuexin, Denis, Deniz,Vijay, Andras, Mirko, Mannon, Pieter, Dae June, Nicolai, Janet, Jordi, Xing Yi, Alberto, Victoria, Raluca, Chie Ching, Huaping, Oktay, Yiping, Lanti and Mudassir.

I would like to acknowledge the role of few people back in India without whose help I could not have made it till here. I would like to thank Prof. Vishnu Kamath, Prof. Nanje Gowda, Prof. G. Nagendrappa, Prof. T. N. Guru Row and Dr. G. Mugesh for their help and encouragement. There were many people who inspired me to pursue a career in pure science. I would like to thank Vatsa, Ram, Anand, Manoj, Sarathy, Praveen, Chandru, Ajay and Shivu in this regard. I would also like to thank Prof. R. Venugopal, Dr. Mehboob Peeran, Prof. G. Swarnalatha, Prof. K.V. Ghanashyam and Prof. B. N. Narayana Reddy for instilling the confidence in me to come this far.

Without my family's support I could not have made it till here. Appa, Amma, Mythri, Raghu, Deshi and Bhava, thank you for your continuous support. Last, but not the least, thank you, Rashmi, for being my strength and inspiration. 


\section{Curriculum Vitae}

Srinidhi Ramachandra was born in Bangalore, India on 17th of October 1980. In 2001, he received Bachelor's degree with Physics, Chemistry and Mathematics from Bangalore University. After that he obtained Master of Science degree in Chemistry in 2003 from Bangalore University. Till 2005 he worked as a junior research fellow at the department of Inorganic and Physical chemistry, Indian Institute of Science, Bangalore, where he worked on Laser Raman spectroscopy.

In 2006, he moved to Enschede, the Netherlands to the Supramolecular Chemistry and Technology (SMCT) group, where he worked as a $\mathrm{PhD}$ candidate under the supervision of Prof. David Reinhoudt and Prof. Luisa De Cola. His work here focused on photoinduced processes in nanoassemblies based on quantum dots and organic and organometallic fragments. The results of his research work are described in this thesis. 